\title{
'Some Birds Sound the Same but Most Sing Different' Exploring Multisensory Place-Attachment and Wellbeing with Former Refugees in Nelson, Aotearoa New Zealand
}

\section{Amber Kale}

A thesis submitted to Victoria University of Wellington

in fulfilment of the requirements for the degree of

\section{Doctor of Philosophy}

School of Geography, Environment and Earth Sciences

Victoria University of Wellington

2021 
Abstract

Through everyday multisensory experiences, individuals familiarise themselves with unique environments, people, rhythms, and routines, and form meaningful sociospatial relationships and emotional place attachments. These relationships are often severed during forced displacement, leading many refugees to feel a sense of loss, grief, and disorientation which can negatively impact upon their wellbeing as they move through and resettle in new places. Feelings of loss and grief may be further compounded by the stress of settling into a new unfamiliar geographic terrain and culture.

Despite the importance that meaningful places can hold for individuals, place is often overlooked in resettlement research, which tends to prioritise the provision of basic needs and practices of social integration. However, in this thesis I suggest that a focus on place and multisensory processes of place-attachment can offer important insight into the emotional challenges and benefits of forced displacement and resettlement, and encourage new ways of supporting former refugees to maintain connections to their homelands, develop meaningful relationships with new people and places, and feel well in their everyday lives.

To explore how former refugees' everyday multisensory experiences shaped their feelings of place-attachment and wellbeing, I facilitated a research project in Nelson, Aotearoa New Zealand. Local reports indicated that refugee mental health problems were increasing in this city, and residents had identified a need for 'alternative' (nonWestern biomedical) therapies in the health system. Building upon an emplaced, relational epistemology and participatory arts-based methodology, the research included preliminary 'scoping' interviews with eleven Nelson resettlement practitioners; and meetings, site-specific interviews, map-making activities, painting workshops and a multisensory art exhibition with twelve female Chin and Kayan former refugees.

The conceptual focus on em-placement in this research was significant as it shifted away from the idea of refugees simply being displaced, to recognise that individuals are always physically situated and affected by their material encounters. My focus on multi-sensory experience was also important, as it enabled participants and myself to move beyond the common five senses of sight, sound, smell, taste, and touch, and 
explore other visceral and proprioceptive ways of feeling, experiencing, and representing our surroundings, which contributed to more nuanced, productive ways of conceptualising wellbeing and disseminating research data.

Key findings from the project demonstrated that participants' feelings of placeattachment and wellbeing were shaped through the evocation of nostalgic and fearful memories of the past, affective emotional responses in the present, and the accumulation of multisensory experiences, memories, and emotions over time. These experiences influenced the girls and women's sense of familiarity, safety, happiness, hope, and belonging in both positive and negative ways. They also influenced their feelings of being in or out-of-sync with local time-structures and rhythms, and shaped how they accessed, used, structured, and negotiated space and time and created unique homely and therapeutic-feeling places. Thus, I argue that everyday multisensory experience and meaningful people-place relationships require greater consideration in resettlement research, particularly as the number of globally displaced peoples reaches an unprecedented high, and New Zealand commits to raising its official UN refugee quota and resettling more newcomers in Nelson and other resettlement cities.

\section{Keywords}

Aotearoa New Zealand, Multisensory experience, Place-attachment, Refugee resettlement, Time, Wellbeing 


\section{Acknowledgements}

To acknowledge the significant time, knowledge, support, and funding that has been invested in this research, I would like to thank the following people and organisations. Firstly, thank you to Victoria University of Wellington (VUW) for providing a full threeyear scholarship, without which I could not have taken the time to pursue my $\mathrm{PhD}$. Thank you also to the Womens' Studies Association NZ / Pae Akoranga Wahine for funding my fieldwork through the 2018 Rosemary Seymour Research and Archives Award, and the VUW Science Faculty and the School of Geography, Environment and Earth Sciences (SGEES) for funding my research dissemination at the 2018 Royal Geographical Society (with the Institute of British Geographers) RGS-IBG conference in Wales.

Further thanks are owed to the helpful SGEES administration team, and to the lecturers and professors who have been kind and supportive in the time and intellect they have shared with me during my time at Victoria. In particular I wish to thank my $\mathrm{PhD}$ supervisors Sara Kindon and Polly Stupples, both of whom have greatly inspired my research and my work ethic throughout my post-graduate studies. Their attention to detail and dedication towards enhancing equitable social outcomes has encouraged me to produce rigorous empirical research, whilst also considering the impact that my work might have on the people and environments with whom I interact on a daily basis. In this manner, they have helped me to apply my theoretical learnings to specific local issues and to view academia as a pathway towards social justice and the development of unique placebased relationships. They have also offered support and friendship throughout my studies, always ensuring that I am well and happy and that my $\mathrm{PhD}$ experience would be a positive one. I have enjoyed the time we shared together presenting at conferences and writing articles, and I hope that we will have the opportunity to collaborate again in the future.

I also wish to thank the resettlement practitioners who supported this project from the Nelson Red Cross, the Nelson Multicultural Centre, Victory Community Centre, English Language Partners, Victory School, Community Artworks, the Nelson Marlborough District Health Board, and through various other places of employment. These individuals played a crucial role in my understanding of resettlement praxis in Nelson, and, despite 
their busy schedules, they made time to answer my questions and to welcome me to the resettlement sector.

Another group of individuals who deserve a huge thank you are my lovely research participants, Mu Pli, Ruth, Little Ruth, Ngai Ciang Sung, Mai Aye Aye, Ngun, Sui Sung, Iang Chin Sung, Elizabeth, Zai Zai, Jenavi, and Sui Tin Men. I wish to thank these girls and women for the time, knowledge, memories, and feelings they shared during our multisensory mapping project, and for welcoming me into the Kayan and Chin communities. I am grateful for the experiences that we got to share together and look forward to spending more time with you all in the future. I also wish to thank participant's family members, for acting as interpreters, welcoming me into their homes, and attending our research exhibition opening night to support their loved ones.

A significant number of other Nelson community members played a key role in helping me to network and recruit participants, and these relationships were central to the success of my research. Thank you to everyone who made time to talk with me over coffee and to reach out through their networks to advertise the project. In particular I would like to thank Jenna Lyall, Kay Sneddon, and Linda Stern for the time they invested in talking to individuals about the project and connecting me to key informants within the local Kayan and Chin communities.

The Red Cross also provided a venue to present the research findings to Nelson resettlement practitioners, whilst Victory Community Centre and the art teachers at Nayland College provided space to facilitate painting workshops for the multisensory mapping project. The Nelson Marlborough Institute of Technology (NMIT), Dunedin City Library, and Victoria University of Wellington also provided space to exhibit the research to the public, and I thank these institutions and individuals for supporting this research and enabling participants and myself to actively engage with local New Zealand communities.

I would further like to extend my thanks to the staff and students who welcomed me to present my research at Glasgow University in Scotland and Northumbria University in England in 2018, and to the organisers, co-convenors, and presenters at the various conferences I attended throughout my PhD. These conferences include the 2018 and 2019 Visual Research Network residencies and conferences, 2018 and 2019 RGS-IBG 
conferences, 2018 and 2019 Centre for Global Migrations conferences; and 2019 UNESCO RILA Spring School. I also wish to thank my three PhD examniners, Lynda Johnston, Jay Marlowe, and Jen Bagelman, for their critical engagement with my research and the insightful reflections they shared for future publications.

Finally, to those of you who gave me places to stay during my fieldwork and various travels, I thank you for your hospitality and good company. To those who encouraged me to continue studying and offered emotional support, including my father Dennis, mother Karen, brother Jonas, and extended family and friends, I thank you for your faith in my ability to achieve my goals. To my sister Jasmin, who shared endless "study sessions" and cups of coffee with me at Milk and Honey, thank you for keeping me laughing. And to my dearest Steven, who had to relocate overseas for work whilst I completed my studies in New Zealand, I thank you for being so patient and understanding. I consider myself infinitely lucky to have such wonderful inspirational people in my life, and I hope that our combined efforts to produce this research can inspire others and have a positive impact for resettling refugees and the wider places and communities of which we are a part. 


\section{Table of Contents}

Abstract and Keywords $\quad 3$

$\begin{array}{ll}\text { Acknowledgements } & 5\end{array}$

$\begin{array}{lr}\text { Table of Contents } & 8\end{array}$

$\begin{array}{ll}\text { List of Figures } & 11\end{array}$

$\begin{array}{ll}\text { Chapter 1: Introduction } & 13\end{array}$

1:1 Context and aims

1:2 Research approach

1:3 Thesis structure

Chapter 2: Multisensory Place-Attachment and Wellbeing

2:1 Theories of place-attachment

2:2 Understanding (multi)sensory experience

2:3 The role of the senses in everyday wellbeing

2:4 Key learnings

Chapter 3: An Emplaced Conceptual Framework

3:1 Contextualising human experience

3:2 Thinking relationally

3:3 An emplaced model of place-attachment and wellbeing

3:4 Key learnings

Chapter 4: A Participatory Arts-Based Approach

4:1 Research epistemology and methodology

4:2 The multisensory mapping project

4:3 Data collation, analysis, and dissemination

4:4 Key learnings 
5:1 Feelings of nostalgia

$5: 2$ Navigating fearful landscapes

5:3 Maintaining a sense of homeliness

5:4 Key learnings

Chapter 6: Therapeutic-Feeling Places and Solitude

6:1 Developing a sense of Nelson

$6: 2$ Seeking therapeutic-feeling places

6:3 A desire for solitude

6:4 Key learnings

Chapter 7: Time, Familiarity and a Sense of Belonging

7:1 Becoming familiar with new places and rhythms

7:2 Finding 'a comfortable place with each other'

$7: 3$ Changing the city and society

7:4 Key learnings

Chapter 8: Feeling Well in Place

8:1 Emplacing refugee mental health

8:2 Harmonising with one's surroundings

8:3 An ethics of responsibility and care

8:4 Key learnings

Chapter 9: Conclusion

9:1 Summary of key learnings

9:2 Theoretical contributions

9:3 Future research and resettlement recommendations 
Appendix One: VUW human ethics approval Appendix Two: Practitioner recruitment letter Appendix Three: Practitioner information and consent forms Appendix Four: Practitioner interview guide Appendix Five: Participant recruitment flyer Appendix Six: Fieldwork schedule Appendix Seven: Participant information, consent, and confidentiality forms Appendix Eight: Participant interview questions Appendix Nine: Research dissemination Appendix Ten: Codebook sample 


\section{List of Figures}

Figure 1:1: New Zealand Resettlement Regions ......................................17

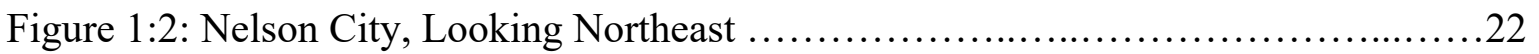

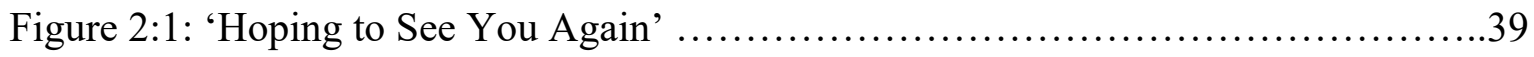

Figure 2:2: 'I Do Need Peace' ....................................................... 40

Figure 2:3: Damasio's Model of Consciousness ....................................4

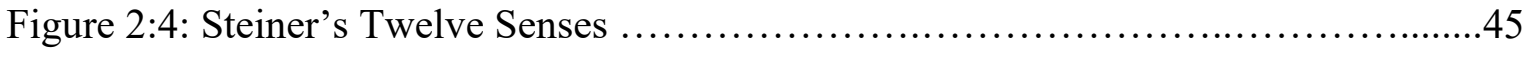

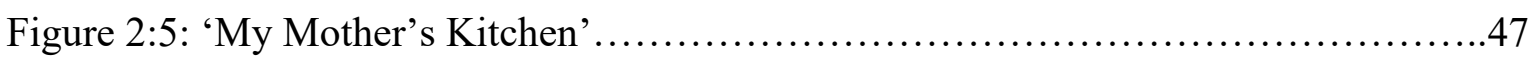

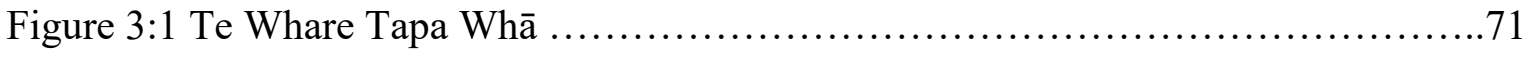

Figure 3:2: The Tripartite Model of Place-Attachment .................................72

Figure 3:3: An Emplaced Model of Place-Attachment and Wellbeing .....................74

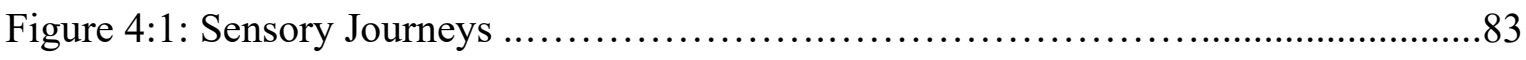

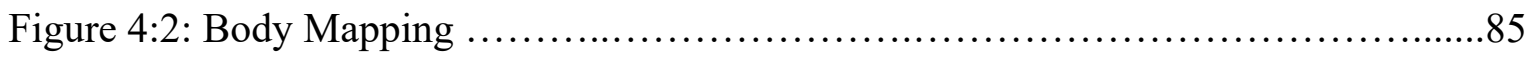

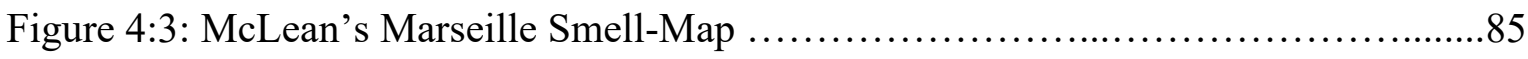

Figure 4:4: Street Map of Nelson City ............................................. 91

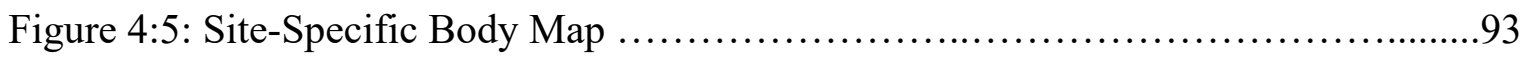

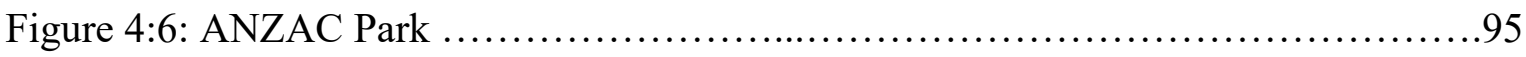

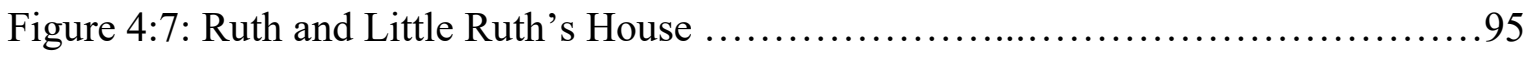

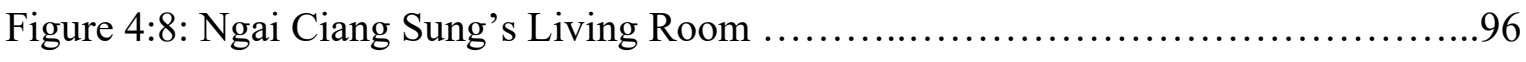

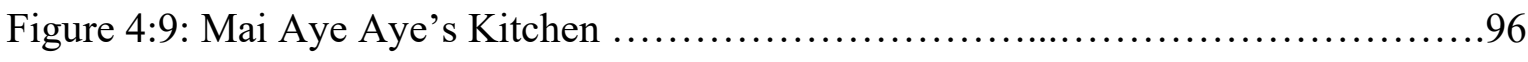

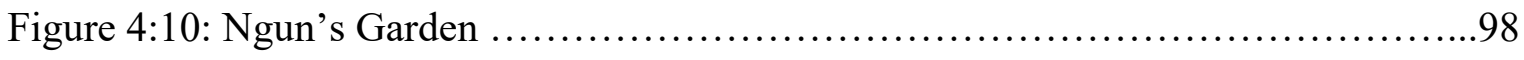

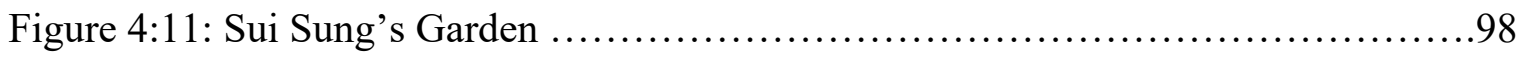

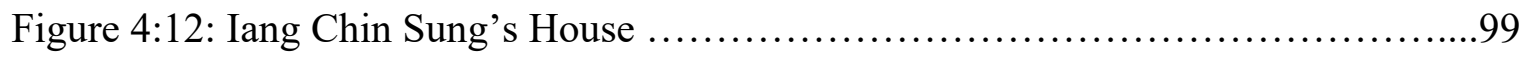




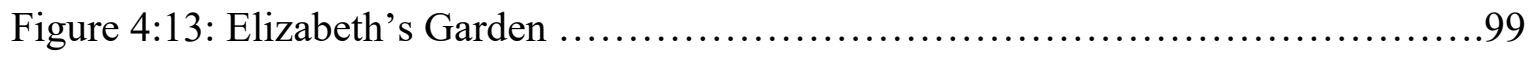

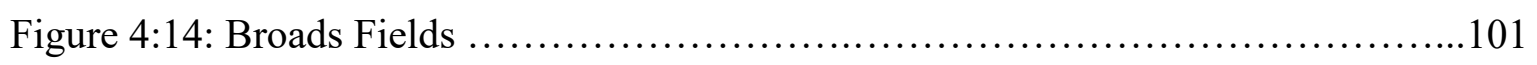

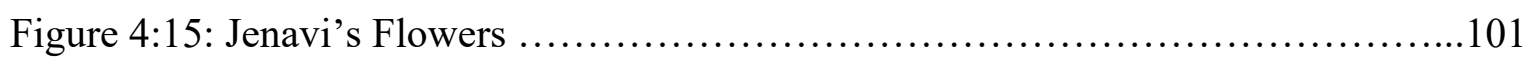

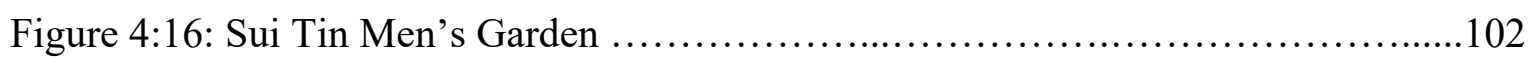

Figure 4:17: Painting at Victory Community Centre ................................. 104

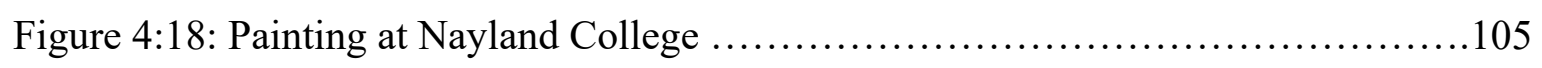

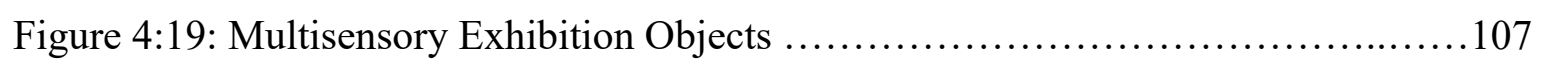

Figure 4:20: The Exhibition at G-space Gallery .....................................109

Figure 4:21: Nelson Exhibition Opening Night ...................................110

Figure 4:22: Exhibition at Dunedin City Library ....................................111

Figure 4:23: Wellington Forum and Diversity Festival Display .......................112

Figure 5:1: Ethnic States and Regions of Myanmar....................................121

Figure 5:2: Feeding Birds at the Queens Gardens …...............................122

Figure 5:3: Walking up the Mountain at Whenua Iti...................................123

Figure 5:4: Playing with the Acorns at Broads Field .................................125

Figure 5:5: Cooking Spicy Food in the Kitchen.....................................142

Figure 5:6: Fishing by Sealords ................................................... 144

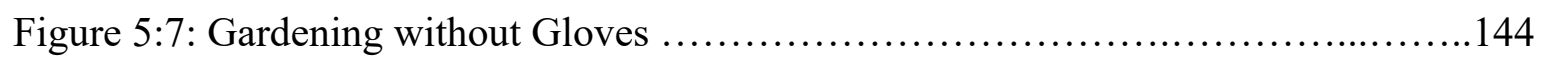

Figure 6:1: Feeling Better in the Garden ..........................................161

Figure 6:2: Watching the Clouds at ANZAC Park ......................................161

Figure 6:3: Enjoying the Green Leaves in the Hills.................................... 164

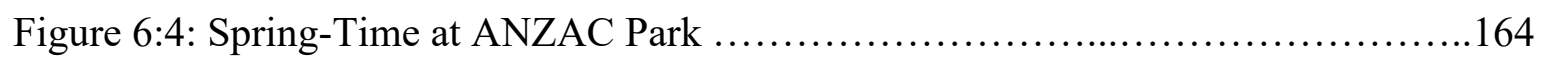

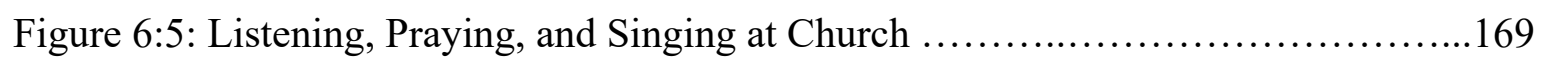

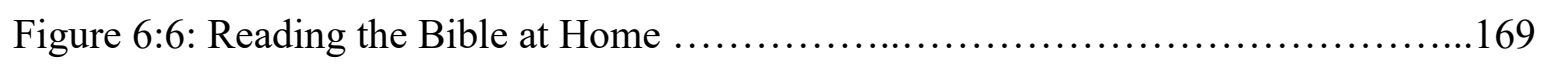




\section{Chapter 1: Introduction}

In this thesis I explore how former refugees' individual multisensory experiences can build emotional attachments to places of settlement and enhance their sense of wellbeing in a new city. Specifically, I draw upon a participatory arts-based project that I facilitated as part of my $\mathrm{PhD}$ research with resettlement practitioners and female Chin and Kayan former refugees living in Nelson, Aotearoa New Zealand. Place-attachment is not currently acknowledged as a vital part of the New Zealand refugee resettlement process, and place is largely absent in local conversations around refugee mental health care. However, I argue that for individuals who have been uprooted from sites of personal and cultural

significance and systems of social support through forced displacement, building strong connections to new places is critically important. Such connections can enable feelings of belonging, happiness, and hope for the future, and ultimately enhance individual wellbeing and provide better resettlement outcomes. In what follows, I introduce my research context, aims, and approach, and outline a guide to the subsequent chapters.

\section{1:1 Context and aims}

\section{New Zealand refugee resettlement}

During the course of my $\mathrm{PhD}$ research, the estimated number of displaced people around the world surpassed 70 million - the highest figure ever recorded (UNHCR, 2019). On their website, the United Nations Educational, Scientific, and Cultural Organisation (UNESCO, 2017) defined human displacement as,

The forced movement of people from their locality or environment and occupational activities. It is a form of social change caused by a number of factors, the most common being armed conflict. Natural disasters, famine, development and economic changes may also be a cause of displacement.

Individuals who are forced to leave their homes generally seek refuge within their own country as an 'internally displaced person' (IDP). However, others may flee to another country, to live amongst the locals or in designated refugee camps until they deem it safe 
to return home or are forcibly repatriated. In many cases returning home is not a viable option, and individuals may therefore decide to seek asylum (protection from the state) in the country in which they now reside. If this is not possible, they may begin the tedious process of applying for refugee status through the United Nations (UN), in the hope that they will be accepted for asylum in a third country which is signatory to the $1951 \mathrm{UN}$ Refugee Convention (UNHCR, 1996). The convention defines a refugee as someone who,

Owing to wellfounded fear of being persecuted for reasons of race, religion, nationality, membership of a particular social group or political opinion, is outside the country of his [sic] nationality and is unable or, owing to such fear, is unwilling to avail himself [sic] of the protection of that country; or who, not having a nationality and being outside the country of his [sic] former habitual residence as a result of such events, is unable or, owing to such fear, is unwilling to return to it.

Despite the outdated use of masculine pronouns, the UN now recognises that this statement applies to all genders. Third country resettlement is often favourable as it may confer residency or citizenship rights and the opportunity for displaced individuals to seek education and employment, build new social networks, and plan ahead for the future. However, in 2016, only 189,300 asylum seekers who applied for refugee status were accepted for official UN resettlement worldwide (UNHCR, 2017).

In response to public campaigning and pressure from national and international rights groups for New Zealand to pull its weight during this crisis of displacement, the Labourled coalition government agreed to double New Zealand's shamefully stagnant UN refugee resettlement quota of 750 places in 2020, raising the total to 1,500 people per annum (New Zealand Government, 2018b; Stephens, 2018). Throughout this thesis I refer to these individuals as 'UN quota refugees'. A further 300 resettlement places are set aside each year for refugees who have relatives residing in New Zealand, as part of a sponsorship system known as the 'family reunification' programme, and asylum seekers can apply for refugee status upon their arrival into the country. In 2017 the government also introduced a new resettlement category called 'Community Organisation Refugee Sponsorship' (CORS), which currently resettles up to 25 individuals each year (New Zealand Immigration, 2018c). 
Whilst asylum seekers are not necessarily guaranteed New Zealand citizenship rights and may not be eligible to reside in New Zealand long-term, UN quota refugees and CORS refugees automatically obtain 'permanent residency' upon their arrival into the country, and those who are sponsored by family have a good chance at becoming permanent residents. This legal status enables individuals to live, work, or study in New Zealand indefinitely, and to vote in all local body and national elections. It also offers the opportunity to apply for citizenship after five years (Immigration New Zealand, 2016; New Zealand Immigration, 2018a). To acknowledge this change in status from being a refugee to becoming a New Zealand resident upon resettlement here, I use the terms 'former refugee' and 'refugee-background' when referring to participants in this thesis.

Once in the country, former refugees follow one of two different pathways to settlement. UN quota refugees initially have a five-week orientation at Auckland's Mangere Refugee Resettlement Centre in which they attend English language classes, health screenings, and mental health support programmes (New Zealand Government, 2018b). They are then resettled in different cities around the country, including Auckland, Hamilton, Palmerston North, Levin, Masterton, Wellington, Nelson, Blenheim, Christchurch, Ashburton, Timaru, Dunedin, and Invercargill (New Zealand Red Cross, 2020a; see Figure 1:1). In these regions, local branches of the New Zealand Red Cross work closely with qualified social workers, case workers, cross-cultural workers and trained volunteers to help quota refugees understand New Zealand culture, navigate local systems, and find work, schools, and doctors (New Zealand Red Cross, 2020a).

In contrast to this state-led model, CORS and family reunification refugees rely on the goodwill of their family and community sponsors to establish themselves in new homes, neighbourhoods, and cities and to integrate into New Zealand society. The CORS programme also relies on the involvement of local churches (New Zealand Immigration, 2018c), reflecting a long history of religious institutions supporting former refugees from all faiths to settle in New Zealand since the Second World War (Beaglehole, 2013).

Despite these extensive national and local support networks, refugees resettling in New Zealand (and other places around the world), continue to face a myriad of challenges in leaving their homes and moving to a new unfamiliar place. Psychologists Rosbrook and 
Schweitzer (2010) argued that the emotional trauma of being separated from one's land, culture, and ancestral connections, and a desperate longing to return to these places, may cause stress and prevent displaced individuals from participating in their new communities and investing time and energy into developing bonds with new places. Other studies have shown that losing an important place may increase the likelihood of developing anxiety and Post-Traumatic Stress Disorder (PTSD), a condition of ongoing mental and emotional duress resulting from an injury or severe psychological shock (Felix et al., 2015; Lonigan et al., 1994). Psychologist Fullilove (2004) also discussed the social, economic, and emotional losses which can trap people in cycles of stress and poverty and compound the initial 'root' trauma of being displaced. My research aims to address these psychological and emotional challenges and consider how New Zealand might enhance resettlement outcomes for displaced individuals as our annual resettlement quota increases.

\section{Approaches to refugee mental health and wellbeing}

As mentioned above, New Zealand's existing resettlement model includes mental health support programmes. The World Health Organization (2005, p. xviii) defined mental health as, "A state of well-being in which the individual realizes his or her own abilities, can cope with the normal stresses of life, can work productively and fruitfully, and is able to make a contribution to his or her community". In contrast, poor mental health implies unrealised abilities or potential and an inability to cope with daily stressors, and is linked to low productivity and social disengagement. Poor mental health may also have implications for how individuals perceive their surroundings and engage or disengage with different places (Jones, 2001; McGeachen \& Philo, 2017; Philo \& Wolch, 2001).

To address poor mental health, Auckland service provider 'Refugees as Survivors New Zealand' (RASNZ, 2018) offers integrated mental health care including psychological assessment, therapeutic interventions, body-therapy, community programmes and initiatives, and tailored support for refugee background youth. 'Refugee Trauma Recovery', which is based in Wellington, also has a team of psychologists, psychotherapists, counsellors, a social worker and a psychiatrist. They offer screening, assessment, treatment, therapy, care coordination and onward referrals to other health practitioners (New Zealand Red Cross, 2018). 
Figure 1:1: New Zealand Resettlement Regions

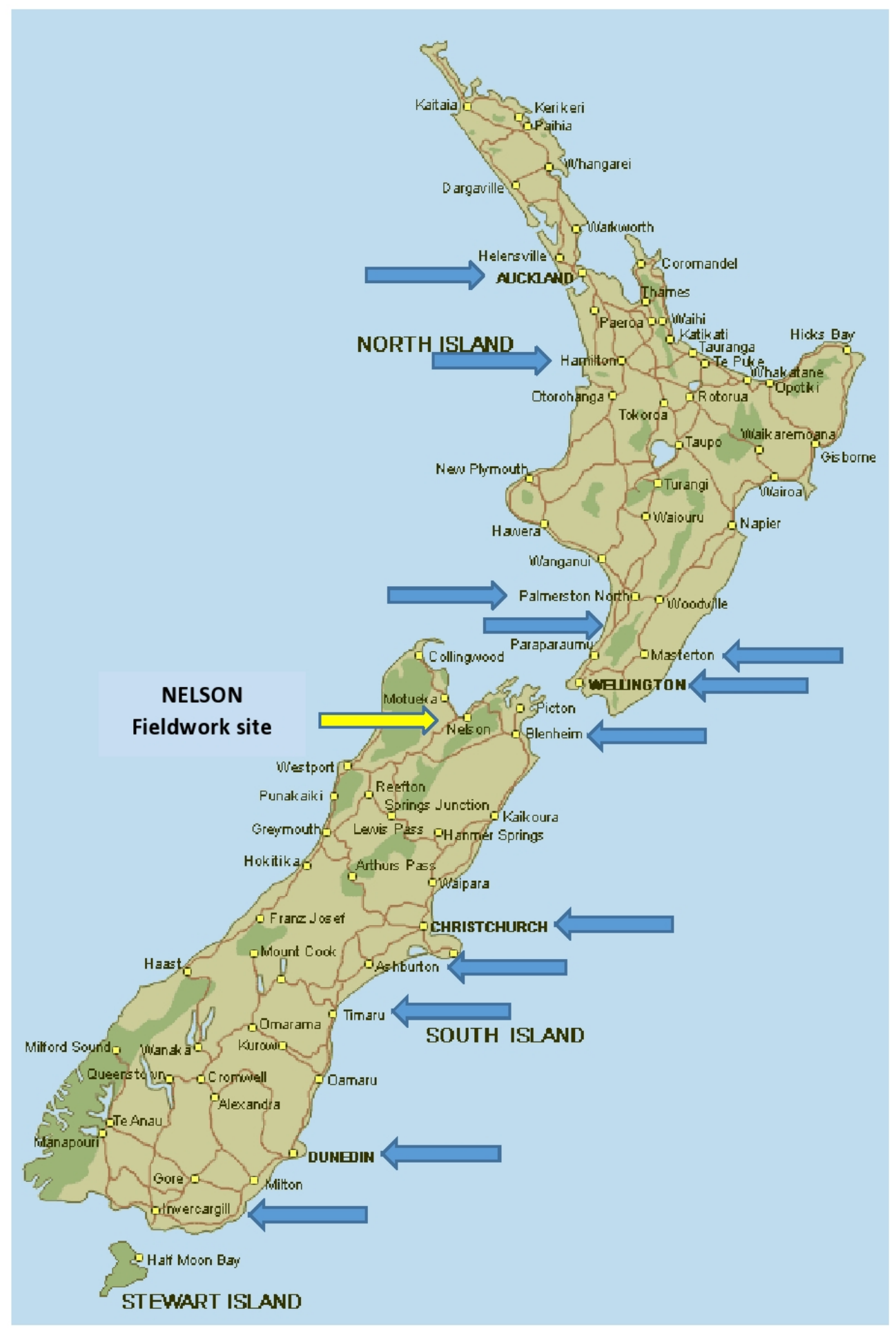

(Source: Map edited from Backpack New Zealand, 2019). 
These specialised services may be useful for displaced individuals; however, by catering to former refugees-only they also run the risk of reinforcing negative stereotypes of refugees as traumatised, vulnerable, and 'different' from the general population (Ford, 2012). On average, only seven per cent of former refugees resettling into New Zealand suffer from PTSD - a number that closely aligns with PTSD rates noted in the wider New Zealand population (6.1 per cent) (Javidi \& Yadollahie, 2012; McLeod \& Reeve, 2005). These relatively low statistics challenge common misconceptions that all former refugees are traumatised, as they show that the majority of newcomers have not been diagnosed as suffering from a stress-related psychological disorder. They also highlight the fact that PTSD is not exclusive to individuals coming from areas of conflict or those who are struggling with forced displacement. However, former refugees may still experience some level of stress and anxiety as a normal human response to their disruptive and uncertain circumstances (Fullilove, 2004; Rosbrook \& Schweitzer, 2010).

In an attempt to avoid the misleading 'traumatised' refugee stereotype and still provide care to those who need it, the Southern District Health Board (DHB) (who cater to a vast geographic region including the cities of Dunedin and Invercargill) has incorporated former refugee healthcare into mainstream services. At the 2018 Centre for Global Migrations' health and wellbeing conference in Dunedin, presenters associated with the Southern DHB claimed that this move supported a 'rights-based' approach to healthcare, where all New Zealanders were offered treatment as equal residents and citizens.

One major flaw with both these specialised and mainstream recovery-based approaches is that services are not sought until after feelings of stress or anxiety have already manifested in depression or impacted an individual's ability to cope with everyday routines and events. Such deficit models operate as the 'ambulance at the bottom of the cliff' rather than acting to prevent or minimise emotional and mental duress (New Zealand Government, 2018a). These services also adopt an individualistic approach to recovery, which places the onus of healing or feeling well on the person suffering, without necessarily addressing the wider environment, circumstances, or relationships which contribute to a decline in mental and emotional health (Andrews et al., 2014; Atkinson et al., 2012; Hinton et al., 2004; Wilson, 2003). 
Smaller, under-resourced resettlement cities, like Nelson, may find it particularly difficult to provide refugee mental health care (Nelson Multicultural Council, 2012; Ward et al., 2018). Geographically situated at the top of the South Island and in the center of New Zealand, Nelson hosts a population of approximately 50,000 (Stats NZ, 2020). The city has been home to former refugees from Asia since the 1970s, and was officially selected as a refugee resettlement city in 2007. There are currently about 700 former refugees living in and around the central business district (CBD), from Cambodia, Vietnam, Myanmar (formerly known as Burma), and Nepal (Asia New Zealand Foundation, 2018). Refugees fleeing from civil war have also recently been resettled from Colombia (Bartlet, 2018). Despite this long history of resettlement, a lack of mental health services was a particular concern raised by resettlement practitioners in the Nelson Multicultural Council report (2012, p. 3), as there were, "Increasing numbers of refugee arrivals with high and complex needs and emerging health issues for longer term residents".

To address this concern, participants in a 2018 Nelson resettlement study recommended, "The inclusion of alternative therapies in the health system" (Ward et al., 2018, p. 23). This recommendation was also reflected in a wider New Zealand Government (2018a) report, entitled 'He Ara Oranga: Report of the Government Inquiry into Mental Health and Addiction'. In this report, New Zealand residents and citizens from around the country called for a more holistic, participatory, and preventative approach to mental health where they could access the necessary skills and resources needed to minimise stress and anxiety and enhance a feeling of comfort and safety in their everyday lives.

A holistic, participatory, and preventative health approach already exists in New Zealand through Indigenous Māori frameworks of wellbeing such as health practitioner Durie's (1985) 'Te Whare Tapa Whā' (The four cornerstones of health) and educator Pere's (1997) 'Te Wheke' (The octopus, whose eight tentacles collectively contribute to 'waiora' or holistic wellbeing). Māori models also consider whānau (family), and wairuatanga (spirituality) to be key components of good health; with a spiritual aspect not only implying a connection to gods or ancestors but also a relationship between people and place. Durie (1985, p. 483) claimed that, "Without access to traditional or tribal land, many 
Māori elders would diagnose poor health, and the health history of Māori people would confirm the central importance of land to health".

This conceptual shift from mental health to wellbeing is significant, as there is an explicit acknowledgement that one's psychological and emotional states are affected by, and capable of affecting, one's entire physical being as well as the external world. To position this relational understanding of human health at the forefront of my research, I also adopt the term wellbeing, further drawing upon geographers Kearns and Andrews (2010, p. 98) description of wellbeing as, "Both being well (i.e., having access to life's material necessities) and feeling well (having the capacity to act and find meaning in the world)".

\section{Research aims and objectives}

In line with holistic Māori healthcare, a focus on family and social support are becoming more common in New Zealand (New Zealand Government, 2018a), and many religious groups have their own spiritual wellbeing practices. However, the role of place in wellbeing remains under-explored. I aim to centralise this missing element in this thesis, specifically focusing on the concept of 'place-attachment' - defined as an emotional bond between an individual and a unique place that holds meaning to them (Lewicka, 2011; Scannell \& Gifford, 2010; Tuan, 1977).

In a study which drew upon a large representative survey in Poland, psychologist Lewicka (2011, p. 218) claimed that,

Place-attached persons, compared to non-attached ones, demonstrated a higher sense of coherence, were more satisfied with their life overall, had a stronger bonding social capital and neighborhood ties, were more interested in their family roots, trusted people more, and were generally less egocentric.

These findings were supported by environmental psychologists Scannell and Gifford's (2016) laboratory study with ethnically-diverse undergraduate students, which found that becoming emotionally attached to a place increased a sense of belonging, self-esteem, and meaning. Whilst these studies did not specifically focus on refugees, such benefits could potentially counteract some of the negative feelings and stressors incurred through experiences of displacement, and provide displaced individuals with the support networks, 
confidence and meaning needed to support their wellbeing.

Scannell and Gifford's (2016) claim that place-attachment may enhance a sense of belonging is particularly significant in terms of meeting the objectives of the 'New Zealand Refugee Resettlement Strategy'. The strategy was collaboratively implemented in 2013 by the New Zealand government, local non-governmental organisations (NGOs), and refugeebackground communities (New Zealand Immigration, 2018b). The main objective is that,

Refugees are participating fully and integrated socially and economically as soon as possible so that they are living independently, undertaking the same responsibilities and exercising the same rights as other New Zealanders and have a strong sense of belonging to their own community and to New Zealand.

Feminist and cultural theorist Probyn (1996, p. 6) claimed that, "Belonging expresses a desire for more than what is, a yearning to make skin stretch beyond individual needs and wants". In this manner, belonging encompasses a 'sensation of longing', a visceral embodied feeling that one is incomplete without meaningful relationships whereby they are intimately connected to another entity - be that a place, person, animal, object, or metaphysical being. This sensation of longing is predominantly felt when one is sociospatially or emotionally isolated and positioned as an 'outsider'. To counteract such isolation, people seek out places and experiences where they feel as though they are part of something greater than themselves, and where they feel safe, protected, valued, and hopeful (Antonsich, 2010). Unfortunately, whilst belonging is evident in policy, it is not always supported in New Zealand resettlement practice, where it is often overshadowed by a focus on employability and national economic growth (McBrien, 2014; New Zealand Immigration, 2018b). This is problematic as individuals who feel that they don't belong are more likely to become socially and spatially isolated and psychologically distressed (Scannell \& Gifford, 2016).

Whilst there already exists a wealth of academic literature on place-attachment (and belonging), geographers Tuan (1977) and Seamon (1980), and psychologist Lewicka (2011), have identified a gap in place-attachment research and discussed the significant role of sensory perception in developing emotional relationships with place. Sensory perception refers to an interactive neurophysiological process, whereby different sights, 
sounds, smells, tastes, textures, and other environmental stimuli are internalised and processed in the neural networks of the brain, enabling individuals to make sense of their surroundings (Baddeley et al., 2009; Damasio, 2000). Such experiences may also be described as multisensory, building on the idea that the senses are integrated in the nervous system (Ingold, 2000; Rodaway, 1994; Tuan, 1993). I agree that a focus on these embodied affective experiences is important in understanding lived experience. However, framing a research question around sensory perception would centralise the subjective self and overlook the wider sociospatial and temporal relationships between people and place (Thrift, 2008). To enable a greater understanding of how external environments, events, and networks might influence individual perceptions, emotions, memories, and behaviours, I therefore adopt the broader term, 'multisensory experience' in this thesis.

Multisensory experiences of place and belonging are beginning to be theorised by New Zealand academics (Bartos, 2013; Longhurst et al., 2009; Trnka et al., 2013); however, my review of recent literature has not identified any New Zealand studies that explore how multisensory place-attachment could inform refugee resettlement outcomes and holistic wellbeing. My thesis aims to explore this nexus, drawing upon the data generated through my participatory arts-based research project in Nelson City (Figure 1:2).

\section{Figure 1:2: Nelson City, Looking Northeast}

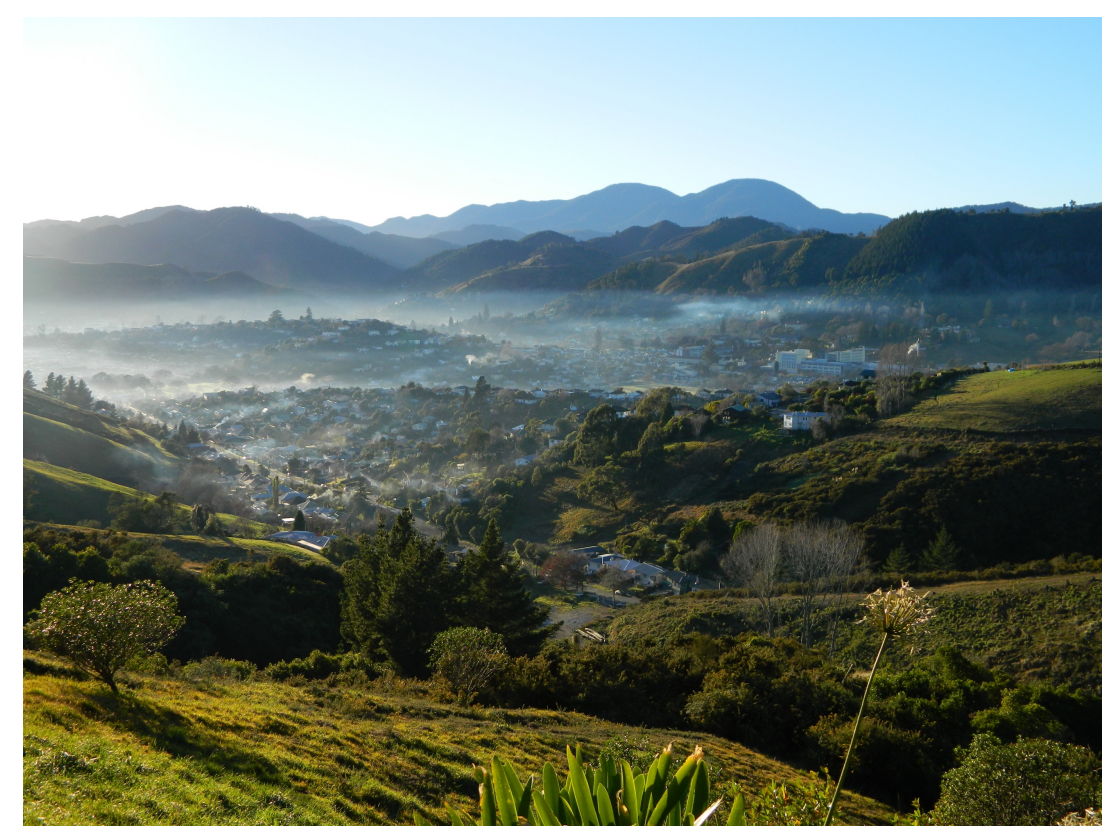

(Source: Photo by author, 2012) 
Nelson was selected as a research site in response to the aforementioned calls by residents for alternative therapies in the health system (Nelson Multicultural Council, 2012; Ward et al., 2018). Selecting this city also enabled me to explore another resettlement location beyond the more populated regions of Auckland and Wellington, where the majority of New Zealand refugee resettlement research is situated (Collie et al., 2010; DeSouza, 2011; Elliot \& Yusuf, 2014; Ford, 2012; Kale, 2017; Kale \& Kindon, 2020; Kamri-McGurk, 2012; Marlowe, 2017; Mortensen, 2008). Nelson is also my hometown, and I hoped that my local knowledge and social networks would enable me to produce in-depth research and contribute to development within my community.

I decided to work with individuals from Myanmar as, over the past decade, New Zealand and Nelson have taken in more former refugees from Myanmar than from any other country (Asia New Zealand Foundation, 2018). Today, around 2,200 individuals born in Myanmar call New Zealand home, with communities also being resettled in Auckland and Wellington (Mon, 2014; New Zealand Foreign Affairs and Trade, 2018). Refugees from Myanmar have further settled in Australia, Canada, the USA, Finland, the UK, the Netherlands, Norway, and Sweden (McKinsey, 2006). Working with a larger community meant that the research could potentially have greater outreach and wider benefits for local refugee resettlement, and it also offered a greater pool of individuals to engage with, which was important considering the past challenges I had faced in recruiting participants from a refugee background for research in Wellington (Kale, 2017). Whilst I initially invited former refugees from any Myanmar ethnic group to participate, those who became involved in the research were from the Chin and Kayan communities.

The decision to work with female participants was then based upon an early review of resettlement research, which indicated that emotional and mental health issues often have gendered dimensions and that refugee-background women are more likely than men to be isolated in their homes, and unable to access social support due to limited access to transport, domestic responsibilities and limited English language ability (Bose, 2014; DeSouza, 2011; Kale \& Kindon, 2020; Martin, 2008; Swe, 2013; Urry, 2012). Local practitioners also suggested that women may be more comfortable speaking with a female researcher, a point I expand upon in Chapter Four. 
This research focus led me to critically explore and discuss the following question:

How do everyday multisensory experiences shape feelings of place-attachment and wellbeing for female Chin and Kayan former refugees in Nelson, Aotearoa New Zealand?

To understand the various factors influencing individual multisensory experience, I drew upon relevant literature to explore neurophysiological processes of sensory perception and memory (Baddeley et al., 2009; Damasio, 2000); sociocultural encounters (Howes \& Classen, 2014; Law, 2001; Tuan, 1991); the use of mobile communication technologies (Elliot \& Urry, 2010; Marlowe, 2017; McNeill, 1994; Ozkul, 2013); geographic processes of place-agency (Ash \& Simpson, 2016; Larsen \& Johnson, 2016; McCormack, 2017), and the beats, rhythms, cycles, and structures of time (Edensor, 2010; Harvey, 1994; Lefebvre, 2004; Potts, 2015; Seamon, 1980). This extensive body of interdisciplinary research guided the development of the following research sub-questions:

1. What role does sensory memory play in shaping participants' feelings of placeattachment and wellbeing? And what role do new experiences play in reshaping sensory memories?

2. What roles do physical, social and virtual environments play in shaping participants' multisensory experiences and feelings of place-attachment and wellbeing?

3. How do multisensory experiences and memories relate to processes of 'othering' and exclusive or inclusive sociospatial practices?

4. How do repetitive engagements with multisensory stimuli influence individual feelings of place-attachment and wellbeing over time?

5. How do multisensory experiences and memories enhance or prevent a sense of belonging somewhere?

The objective of this research was threefold, with an emphasis on exploring unique methods and ways of 'doing' multisensory research (Pink, 2015); enhancing knowledge in the fields of place-attachment and wellbeing; and applying this knowledge in a real world setting. Specifically, I aimed to contribute to an understanding of how individuals experience the world through the senses; the impact that these multisensory experiences 
can have on individual relationships with place and feelings of wellbeing; and the practical implications of facilitating multisensory research and sharing this research with others. I aimed to build upon these theoretical understandings to contribute to local Nelson and New Zealand conversations around alternative (non-Western biomedical) approaches to refugee wellbeing, and to consider how we, as individual residents and a collective nation, might enhance resettlement outcomes and develop a more holistic resettlement model.

Whilst this thesis is unique in combining elements of New Zealand refugee resettlement, multisensory place-attachment, and holistic wellbeing, it fits into a growing body of international and interdisciplinary research on former refugees' sensory and emotional experiences of place (Biglin, 2020; Coughlan \& Hermes, 2016; Kale et al., 2019; Munt, 2016; O'Neill \& Hubbard, 2010; Sampson \& Gifford, 2010). Thus, my research can contribute a New Zealand perspective on the lived experience of resettling refugees and play a small role towards generating more positive resettlement outcomes internationally. This thesis also contributes to the conceptual expansion and integration of existing sensory frameworks, broadening the breadth of stimuli and affective emotional experience being theorised within social science research. I also draw attention to the temporalities of everyday emplaced experience, focusing on the social and seasonal cycles, rhythms, and routines which characterise place and impact former refugees' ability to settle and build meaningful sociospatial relationships.

\section{1:2 Research approach}

\section{Epistemology and methodology}

To understand the relationship that participants had with different places in Nelson, I adopted an 'emplaced' conceptual framework (Casey, 2009; Pink, 2015; Seamon, 2018). This framework contextualised embodied multisensory experience within a wider sociospatial, temporal, and 'more-than-human' (Whatmore, 2006) environment, enabling me to consider how participants' dynamic interactions with other people, terrain, flora, fauna, objects, and technologies influenced their affective, emotional experiences. 
Whilst I focus on experience in Nelson, and participants' everyday material interactions, I view place as spatially and temporally 'open' (Massey, 2005), forever being (re)constructed through complex relationships, events, networks, and flows (Amin, 2004; Wright, 2015). Therefore, I also consider how the girls and women's experiences are shaped by their ongoing plurilocal and transnational connections (and memories of home), as well as Nelson and New Zealand's wider geopolitical links. In this manner, my work weaves together various scales of place, exploring how global, national, and local events are affectively felt by, and shaped by, individuals as they go about their daily lives and routines (Seamon, 1980; Thrift, 2008).

In order to experience for myself the different places and multisensory experiences that held meaning for participants, I adopted a participatory methodology (mrs kinpaisby, 2008; Pain \& Kindon, 2007), where I could actively engage in the research process alongside participants and become 'similarly situated' (Pink, 2015). This approach led to the design and implementation of a participatory arts-based project entitled 'the multisensory mapping project'. In June 2018 I interviewed eleven refugee resettlement practitioners in Nelson to see what initiatives were in place to assist former refugees to settle comfortably into their new city. The following January, I interviewed twelve Chin and Kayan girls and women in places to which they felt attached in Nelson.

Being in these places together meant that I was able to move beyond traditional oral, written, and visual forms of knowledge construction and feel the affective multisensorality of the moment. However, traditional oral interviews remained important in sharing these affective experiences intersubjectively. To overcome some of the oral language barriers that are often present in cross-cultural research, and to communicate multisensory experience in a manner that was, "Closer to the multidimensionality of the subject itself" (MacDougall, 2005, p. 116) we also incorporated visual and tactile methods of mapmaking and painting (Ferguson, 2011; Ramos, 2004) so that participants could share their subjective place-based experiences. Yet, whilst I had found painting to be an effective communication tool in the past (Kale, 2018), participants in my Masters' resettlement research had also claimed that, "We could easily just draw a stick figure and everything, but like, how we were talking about how much smell can relate to home, or how we connect, so we couldn't draw that but it's something that we all talked about'. 
To disseminate a wider array of multisensory experiences in this study, I exhibited participants' paintings alongside related three-dimensional objects with unique visual, auditory, olfactory, gustatory, cutaneous, and other sensory qualities (Exploratorium, 2018; McClean, 2017). These objects enabled an immersive hands-on approach whereby the Nelson community could also engage with participants' journeys in an embodied relational way and develop a more empathic intuitive understanding of their experiences. This was important in deconstructing negative refugee stereotypes and misrepresentations and encouraging people to understand one another and connect on an emotional level. This research approach and the unique research methods are discussed in more depth in Chapters Three and Four.

\section{Researcher positionality}

Due to my active participation within research processes, self-reflexivity was an important aspect of producing rigorous, ethical research (Cahill et al., 2007; Kindon, 2012). Anthropologist Pink (2015, p. 28) claimed that, "It is now frequently recognised that we need to investigate both the emplacement of the people who participate in our ethnographic research and ethnographers' own emplacement as individuals in and as part of specific research contexts". Note that the term 'ethnography' here is common in anthropology, whereas, as a geographer, I later refer to 'empirical' research. To consider how my own multisensory experience might influence the design, conduct, and analysis of this research, I took Pinks' advice and recorded a reflexive piece of writing before I began the multisensory mapping project. I also recorded my own multisensory experiences throughout the practical stages of my research, and later analysed these reflections alongside the data generated by participants, weaving my experiences into this thesis.

It is important to note that I myself am not a refugee, and as a fifth generation Pākehā (of European ancestry), and a New Zealand citizen by birth, I do not know how it feels to be part of a minority group or to face ethnic, religious, or other forms of violent persecution. I also do not know how it feels to be forcibly displaced from my country and community and unable to return home. Drawing upon participants' lived experiences is therefore pivotal to knowing what it means to form emotional place-attachments in the context of forced displacement and resettlement. However, I have travelled extensively in Europe, 
Africa, Asia, and other parts of the world, and know well the disorientation that comes from emerging into an unfamiliar climate and terrain and being overwhelmed by new sights, sounds, smells, flavours, and textures. Thus, in some ways I have been able to relate to participants' multisensory and emotional experiences of moving to a new country.

I have also spent eight years working in the New Zealand refugee resettlement sector, resettling a former refugee family through the Red Cross, campaigning to raise New Zealand's annual UN resettlement quota, evaluating a refugee women's driving programme for ChangeMakers' Refugee Forum (Kale \& Kindon, 2020), helping to organise and attend various refugee-background conferences and events, and facilitating creative research projects (Kale, 2017). This practical engagement has afforded me unique insight into refugee-related experiences, challenges, and opportunities, in the capacity of a friend, activist, artist, and researcher. Once again, these experiences do not make me an expert on the lived experience of others. However, they have taught me the importance of moving beyond abstract theorising and discursive analysis (McCormack, 2005; Thrift, 1996; Vannini, 2015) to understand people and places through the everyday experiences and relationships that make them who they are, and make their lives meaningful.

\section{Ethics of representation}

Despite this immersive reflexive research practice, there still remained the question of how to represent participants' and my own embodied research experiences in conventional academic text. Particularly problematic is the fact that, in writing this thesis, people, places, and feelings that initially required no name, language, or cultural framework to be viscerally and emotionally understood, have now been translated into symbolic words. These words are each laden with unique connotations and nuances and will ultimately be interpreted in a variety of ways (Bourdieu, 1991; Phipps \& Kay, 2014; Tuan, 1991). Thus, the reader will not be sensing what myself and participants experienced, or engaging with our exhibition objects, but rather relying on an interpretive metaphorical account of what different senses were like and why they were significant.

In an attempt to address this challenge, I have incorporated verbatim quotes in my writing, where participants use their own words to communicate their thoughts and feelings. I also support participants' stories with photographs of their paintings and include some of my 
own research reflections. Admittedly, this descriptive, visual account still deprives the reader from knowing the smells, tastes, sounds, and movements of which we speak; however, written and visual communication can also elicit multisensory memories and enable the reader to remember or imagine a time, place, or event that evoked similar feelings within themselves (Bartos, 2013; Kale, 2017). In this manner, even the traditional rigidity of a written thesis can encourage an emotive, empathic human understanding of the experience of others, to some degree.

The writing of this thesis also highlighted the ethical implications of using language that was politically-charged or imbued with negative connotations. Amidst the current crisis of displacement, refugees have been unfairly labelled as economic migrants who pose a threat to local jobs; 'terrorists' who pose a threat to public safety; and 'burdens' who depend on tax-payers to support them without contributing to society (Kirkwood et al., 2013; Tsoukala, 2016). Due to the stigma that the refugee label can bring, participants had the option of remaining anonymous and using a pseudonym in research dissemination. However, anonymity was not enforced as I anticipated that some individuals may wish to take credit for their research contribution, or to acknowledge that being a refugee was a significant part of their life and remains a part of who they are becoming. Talking through these options during our initial meetings, all of the girls and women chose to waive anonymity and use their real names in the research.

Another highly politicised term is the country name 'Myanmar'. In 1989 the ruling military junta officially changed the name Burma to Myanmar, arguing that Burma was a colonial legacy which referenced the country's largest ethnic group and was exclusive of the multiple other ethnic groups comprising the nation. However, because this name change occurred without the consent of the people, some foreign countries such as the UK and USA considered it illegitimate and have retained the use of the name Burma (The Economist, 2016; United States Institute of Peace, 2018). Throughout this thesis I reference the name Myanmar as it is more commonly accepted by the international community, including New Zealand where my research is situated. However, the research participants use these different names interchangeably, providing an important reminder of the complex identity politics at play. 
The issue of ethnic representation in Myanmar also raised questions as to how to collectively refer to participants. The common narrative of being a former refugee and being 'from-Myanmar-but-living-in-Nelson' has led the New Zealand government to construct a pan-ethnic identity which merges diverse ethnicities into a single 'Burmese' demographic group (Asia New Zealand Foundation, 2018). Such ethnic amalgamations are common in New Zealand. Before British colonisation Māori lived in distinctive Iwi (tribes), though are now often perceived to be pan-ethnically united as 'Indigenous peoples' - differentiating them from the Pākehā settlers (Durie, 1998; O’Regan, 2001). In a similar manner, Pākehā were comprised of various European nationalities and ethnicities, but united through their common non-indigeneity. This tendency to 'lump together' unique groups is problematic as it oversimplifies diversity and obscures internal identity politics. Throughout my research it was evident that divergent historical roots and ongoing ethnic tensions in Myanmar have led individuals and communities to dissociate themselves from a national 'Burmese' identity and instead affiliate with their tribal roots (Smith, 1994). So as not to homogenise these groups, I refer to individual participants by their chosen Chin and Kayan ethnic affiliations in recognition of their unique personal and cultural journeys.

\section{1:3 Thesis structure}

To guide the reader through the remainder of this thesis, this section outlines the thesis structure and provides a brief overview of each chapter. In Chapter Two I review interdisciplinary literature on multisensory place-attachment and wellbeing. Drawing upon fifty years of research in geography and environmental psychology, I explore the emotional, cognitive, and behavioural processes through which individuals build meaningful attachments to place (Buttimer, 1980; Lewicka, 2011; Scannell \& Gifford, 2010; Seamon, 1980; Tuan, 1977). I then engage with phenomenological and neurophysiological theories of sensory perception (Damasio, 2000; Merleau-Ponty, 1962) and an expanding field of (multi)sensory scholarship in the social sciences (Edensor, 2010; Hayes-Conroy \& Hayes-Conroy, 2010; Law, 2001; Longhurst et al., 2009; Pink, 2015; Porteous, 1990; Rodaway, 1994; Smith, 1994; Spinney, 2006). I conclude by highlighting the link between the senses, feelings of place-attachment, and holistic wellbeing. 
In Chapter Three I expand upon my 'emplaced conceptual framework', contextualising everyday multisensory experience within dynamic sociocultural (Askins, 2016; Harvey, 1996; Howes \& Classen, 2014; Law, 2001; Tuan, 1991), more-than-human (Bawaka Country et al., 2016; Larsen \& Johnson, 2016; Whatmore, 2006), spatial (Ash \& Simpson, 2016; McCormack, 2017), and temporal (Massey, 2005; Seamon, 1980) events and relationships. Drawing upon a substantial body of work on 'relational geographies' (Amin, 2004; Jones, 2009; Massey, 2005; McCormack, 2005; Thrift, 2008), I develop a conceptual model to analyse place-attachment and wellbeing as intersubjective, emerging between people and places and always subject to change.

Chapter Four then directs the reader through my research epistemology, methodology, and methods, drawing upon the emplaced, relational ontology discussed in Chapter Three, and adopting a participatory arts-based approach whereby the researcher and participants actively co-participate in creative research practice (Kale, 2018; mrs kinpaisby, 2008; Pain \& Kindon, 2007; Tolia-Kelly, 2008; Wynne-Jones et al., 2015). I introduce my aforementioned 'multisensory mapping project', discussing a series of preliminary 'scoping' interviews with refugee resettlement practitioners; and meetings, site-specific interviews, map-making activities, painting workshops and a multisensory art exhibition with the Chin and Kayan former refugee participants. I also discuss data transcription, coding, analysis, and dissemination.

Drawing upon the data generated through the multisensory mapping project, Chapter Five explores how the stimulation of particular senses can elicit memories of past times and places (Blunt, 2003; Hinton et al., 2004), enabling individuals to connect to multiple places simultaneously and form transnational or plurilocal place attachments (Sampson \& Gifford, 2010). I discuss the importance of former refugees being able to mediate between different times and places, and (re)create familiar multisensory environments from home that are intertwined with their sense of self and identity.

In Chapter Six I explore how participants' everyday multisensory encounters with Nelson influenced their feelings towards different places in and around the city. Specifically, I discuss the unique multisensory stimuli that made the girls and women feel scared, stressed, safe, healthy, and happy. I also discuss the therapeutic-feeling places (Conradson, 
2005; Gesler, 1992), and moments of solitude (Long \& Averill, 2003) that enabled participants to escape stressful environments and rest and rejuvenate (Phipps, 2019b). I suggest that these therapeutic-feeling and solitary places are significant in terms of emotional renewal and being and feeling well in place.

Chapter Seven then moves beyond the evocation of sensory memories which draw upon the past, and fleeting multisensory experiences in the present, to consider the repetitive ways in which individuals engage with environmental stimuli over time. I argue that, whilst fleeting multisensory experiences can attract an individual to a place, repetitive engagements and interactions are key to developing a sense of familiarity (Fullilove, 1997; Hage, 1997; Seamon, 1980), pride (Morrison, 2016), and belonging (Antonisch, 2010), and building more meaningful long-term attachments.

In Chapter Eight I draw upon participants' assessments of current Nelson mental health care, and argue for a more holistic, emplaced wellbeing approach which contextualises the sensing feeling human body within wider sociospatial events and relationships (Andrews et al., 2014; Atkinson et al., 2012; Durie, 1985; Wilson, 2003), as well as temporal rhythms and routines (Edensor, 2010; Potts, 2015; Seamon, 1980). I explore the where, when, and how of refugee wellbeing, before discussing an ethics of responsibility and care (Askins, 2016; Fisher \& Tronto, 1990; Lawson, 2007; Popke, 2006), and questioning who is responsible for implementing and maintaining a decentralised relational wellbeing approach in Nelson and other resettlement cities.

In Chapter Nine I revisit my main research question and distil the key learnings, arguing that everyday multisensory experience plays a pivotal role in feelings of place-attachment and wellbeing, and that participants' multisensory encounters in Nelson heavily influenced the types of places which they remembered, and with which they chose to engage, (re)create, (re)imagine, and work towards achieving. I also discuss the academic contribution of my research, in particular the conceptual expansion of existing sensory frameworks and my focus on the temporalities of place-attachment and wellbeing. I conclude with recommendations for future study and resettlement practice, paving the way for applied social research and local initiatives that can further enhance resettlement outcomes. 


\section{Chapter 2: Multisensory Place-Attachment and Wellbeing}

To contextualise my research within existing academic literature, I reviewed interdisciplinary theories on place-attachment, (multi)sensory experience, and wellbeing. Drawing upon almost fifty years of research in human geography and environmental psychology, this chapter highlights the dynamic emotional, cognitive, and behavioural processes through which individuals form and maintain meaningful relationships with their physical and social environments. Acknowledging the central, yet, often neglected role of the senses in processes of place-attachment, I further contextualise this discussion within geography's 'sensory turn', weaving in key neurophysiological theories on sensory perception and diverse geographic, social and cultural frameworks for analysing the senses. This chapter ends with an exploration of the impact of the senses on individual wellbeing, considering how everyday environmental stimuli and the memories they evoke might negatively or positively influence feelings of comfort and a sense of belonging.

\section{2:1 Theories of place-attachment}

\section{Building meaningful relationships with place}

Human geographers and environmental psychologists first began writing about placeattachment in the late 1970s and early 80s (Buttimer, 1980; Relph, 1976; Seamon, 1980; Stokols \& Shumaker, 1981; Taylor et al., 1985; Tuan, 1974, 1977). Studies primarily explored individual or group attachments to residential neighbourhoods and local communities; however, this focus on neighbourhood ties was later critiqued for obscuring the diverse array of places that people encounter in their daily lives and consider to be meaningful (Hidalgo and Hernandez, 2001; Lewicka, 2011; Manzo, 2005). Researchers have since explored attachments to bedrooms and libraries (Sampson \& Gifford, 2010), bathrooms (Manzo, 2005), mountains and rivers (Morse \& Mudgett, 2017), coastlines (Bell et al., 2015), the climate (Knez, 2005), and the virtual world (Coulson et al., 2019; Plunkett, 2011), amongst a myriad of other places.

When considering how meaningful people-place relationships develop, geographers focused on the role of emotional experience. Being in a setting which makes one feel safe, 
relaxed, or happy can foster positive place-based experiences and associations and influence an individual's ability to love and feel proud of a place. These emotional associations were central to the work of geographer Tuan (1974), who described placeattachment through the concept of 'topophilia', which derives from the Greek topos 'place', and -philia 'love of' (the love of place). Both Tuan and geographer Relph (1976) argued that emotional ties fulfil a fundamental human need, in the same way that falling in love with another human does.

Almost thirty years later, environmental psychologist Manzo (2005, p. 67) also acknowledged the importance of emotion in place-attachment; yet, argued that research had been overtly positive and failed to account for the negative and ambivalent experiences that are entangled in feelings of attachment (though she acknowledged that Relph mentioned the potential for place to feel oppressive or restrictive). She claimed that, "We must learn more about the full spectrum of people's experiences in places if we are to understand the complex and multi-faceted phenomena that comprise our emotional relationships to places". Feminist geographers and researchers exploring the concept of home have also noted that meaningful places may be sites of violence or fear and require a more nuanced approach (Blunt \& Dowling, 2006).

Developing an attachment to a place has also been recognised as a cognitive process. Over time, individuals build up memories of people, places, important personal events, and significant milestones which help them to become familiar with their sociospatial surroundings and develop feelings of safety, homeliness, and belonging (Buttimer 1980; Fried, 1966; Fullilove, 1997; Seamon, 1980). Psychologist Fried (1966, p. 363), claimed that, "Even familiar and expectable streets and houses, faces at the window and people walking by, personal greetings and impersonal sounds may serve to designate the concrete foci of a sense of belonging somewhere”. Meanwhile, geographer Buttimer (1980, p. 31) talked about how familiarity with temporal cycles was important. She claimed that, "'Home' for me should ideally have those qualities of my own home - quietness, fragrance, spaciousness, and rhythmic flow of light and dark, winter and spring”. 
Physical mobility is integral to these processes of familiarity, enabling individuals to move through time and space and experience different landscapes (Seamon, 1980). Yet, movement can also be disruptive. Sociologist Gustafson (2014, p. 38), claimed that,

Humanistic geographers, environmental psychologists, and community sociologists have often regarded place attachment as good and mobility as potentially bad. Local roots, community ties, and strong emotional bonds with one's home place have been described as important sources of well-being, whereas mobility has been associated with uprootedness and social disintegration.

Recent studies have been more receptive to mobility, recognising that people constantly move by commuting between home and work, taking walks in the park or summer vacations, shifting to new neighbourhoods, or migrating overseas (Gustafson, 2014). Studies suggest that some people may also be attached to movement itself; due to the positive emotions that can be evoked when walking, cycling, or driving through place (Urry, 2007).

In addition to movement, familiarity relies on an encounter (or some form of contact with) people and places. Evolving from the Latin 'incontrare' (contrary to), encounter has historically referred to a face-to-face meeting between opposing individuals or groups, leading geographer Wilson (2017) to caution that the notion of encounter is 'coded' and 'conceptually charged'. However, encounters are also moments of chance whereby individual pathways intersect. These chance encounters are constitutional in the making of places, boundaries, and identities, and central in the co-construction of difference (Ahmed, 2000; Wilson, 2017), as well as the recognition of that which is similar (Askins, 2016; Noble, 2009; Wright, 2015). Thus, encounters have the potential to be emotionally transformative, in both negative and positive ways (Askins \& Pain, 2011).

Whilst often fleeting (Lawson \& Elwood, 2014) or 'passing' (Laurier \& Philo, 2006), encounters can also be recurring as people develop daily habits and routines which coincide with the habits and routines of others (Buttimer, 1980; Fullilove, 1997; Seamon, 1980; Tuan, 1976). Tuan (1976, p. 274) claimed that habitual behaviour is important in enhancing feelings of place-attachment as, "Habit is biologically adaptive. Tasks, once learned, can be performed unthinkingly so that thought is freed to explore and respond to 
new challenges". Seamon (1980, p. 159) suggested that habitual routines can also pave the way for new social relationships by generating "a sense of familiarity and friendliness" between those individuals whom regularly meet. Geographers have also recently begun to consider the multisensory ways in which people come to know one another and feel their presence (Spinney, 2015), and the importance of encounters with non-human entities, including animals (Gorman, 2017; Hinchliffe \& Whatmore, 2006; Wolch, 2002) and material objects (Bridge \& Smith, 2003; Swanton, 2016).

As individuals familiarise themselves with place and accumulate meaningful experiences and memories, place becomes interwoven with their identity (Proshansky et al., 1983; Twigger-Ross \& Uzzell, 1996). These place-based identities may then be passed on through the generations (Hay, 1998), as children acculturate into local practices, social networks, and historical narratives of place. Such ties are evident in the Māori concept 'turangawaewae' - which is translated as 'a place to stand' and understood as an individual's foundation, their place in the world, or their home as inherited through their whakapapa (ancestral lineage) (Durie, 1985; Hatcher, 2016; Pere, 1997; Reid et al., 2017). Ancestral ties to place are also evident in interdisciplinary researchers Franco et al.,'s (2015, p. 13) book 'The Meaning of Land in Myanmar'. A farmer in their study stated that,

Land carries the history for us. In our grandparents' era, the revolution council era, they fought for the country and got the land. Then they lived here and worked on this land, and they passed the land as inheritance to their later generations. Land is like a remembrance of them.

To enhance positive place-based feelings and spatial identities, people make an effort to maintain proximity to meaningful places, choosing to live there or visit often (Hidalgo \& Hernandez, 2001). They also begin to invest in place, to buy, build, or renovate properties, plant trees and gardens, join social groups, and participate in public life. Through these financial, physical, and emotional investments, individuals may develop a sense of ownership or entitlement and the view that that long-term or socially engaged residents have a more authentic claim to belonging than newcomers or short-term dwellers (Hay, 1998; Porteous, 1976; Relph, 1976; Steadman, 2006). However, researchers exploring short-term relationships with place have claimed that residence time or familiarity is not a reliable indicator of attachment, and that a fleeting impression of a place might be enough 
to impact an individual's decision to return to or settle in that area and encourage them to become actively involved in society (Kale et al., 2019; Lewicka, 2011).

\section{The 'emotional turn' in geography}

In the early 2000s place-attachment gained significant traction with the 'emotional turn' in geography (Anderson \& Smith, 2001; Davidson et al., 2005). In their book 'Emotional Geographies', Davidson, Bondi, and Smith (2005, p. 3) argued for, "A non-objectifying view of emotions as relational flows, fluxes or currents, in-between people and places rather than 'things' or 'objects' to be studied or measured". They claimed that, "An emotional geography, then, attempts to understand emotion - experientially and conceptually - in terms of its socio-spatial mediation and articulation". This focus on relational flows linked into a wider field of 'relational geographies' (Cresswell, 2013; Massey, 2005; Thrift, 2000; Wright, 2015), which I expand upon in the following chapter.

Thinking about emotions relationally challenged researchers to consider not only how places influenced individual feelings, memories, thoughts, and behaviours, but also how these emotional transformations influenced place and altered one's spatial relationships. Geographers Davidson and Milligan (2004, p. 524) claimed that, "Without doubt, our emotions matter. They have tangible effects on our surroundings and can shape the very nature and experience of our being-in-the-world".

Following this new relational trajectory, the notion of 'affect' has also gained prominence. Drawing on the work of geographers Thrift (2004), Anderson (2006), and McCormack (2008), Cresswell (2013, p. 230) claimed that, "Affect differs from emotion in that affect comes in some sense before emotion. Emotions, in this rendering, are ways in which individual bodies make sense of affect by giving it a meaning that is partly socially and culturally determined". Affect could therefore be described as an initial bodily response to one's environment, whilst emotion is the expression and articulation of one's feelings and a way of communicating with others.

Over the past two decades, a substantial body of research has emerged in geography, exploring diverse affective and emotional experiences of hope and fear (Pain \& Smith, 2008; Wilson, 2008), pride and shame (Morrison, 2016), curiosity (Phillips et al., 2015), enchantment (Geoghegan \& Woodyer, 2014), happiness (Scoffham \& Barnes, 2011), loss 
and nostalgia (Blunt, 2003), and belonging (Antonisch, 2010), to name but a few. Analysing these experiences can help researchers to understand more about the types of environments and events which are conducive to place-attachment and wellbeing, or which may pose challenges and leave individuals feeling displaced, unwell, or as though they don't belong.

\section{Attachment in the context of displacement}

Because individual experiences, memories, habits, routines, identities, social networks, and material investments are connected to place, a threat to that place or disruption to one's way of life can be extremely distressing (Buttimer, 1980; Lewicka, 2011, Parr, 2010; Seamon, 1980). Historian Parr (2010, see cover page) said, "Our bodies are archives of sensory knowledge that shape how we understand the world. If our environment changes at an unsettling pace, how will we make sense of a world that is no longer familiar?" This is a particularly poignant question when it comes to refugee resettlement, and something that myself and research participants grapple with throughout this thesis.

In a study with displaced residents from a slum in Boston's West End, psychologist Fried (1963, p. 360) claimed that the loss of a meaningful place could result in feelings of grief. He said, "At their most extreme, these reactions of grief are intense, deeply felt, and, at times, overwhelming”. Geographer Buttimer (1980, p. 14) also considered a threat to a meaningful place to be troubling as, "It appears that people's sense of both personal and cultural identity is intimately bound up with place identity. Loss of home or losing one's place may often trigger an "identity crisis"'. This was supported by a second farmer in interdisciplinary researchers Franco et al.'s (2015, p. 8) book, who said, "Land has very deep meaning and value for us. It is our lives and the very blood in our veins. Without our land, our nationality will vanish. Land is our dignity”.

These experiences of loss and grief dominate the academic and non-academic literature on refugee place-attachments, and forced displacement has been argued to affect individual health and wellbeing and impact one's ability to settle and establish foundations in a new place (Deutsch, 2005; Felix et al., 2015; Fried, 1966; Fullilove, 2004; Lonigan et al., 1994; Rosbrook \& Schweitzer, 2010). In Australian Thoo Lei Wild Grass' (2011) poetic ode to home, entitled 'Hoping to See You Again' (see Figure 2:1), references to the land, kinfolk, 
peaks, rivers, towns, and climate in Karen land (Kayah State, Myanmar) show the author's longing to return to a unique environment which is imbued with their personal history, ancestry, and cultural identity. Similarly, in Kayin resistance writer Tee Noe's (2014) poem, entitled 'I do Need Peace' (see Figure 2:2), the author struggled to come to terms with the fact he will never again see the places where he was born, where he grew up, and where he fell in love. These poems support the ideas that ancestral ties (Hay, 1998) and important personal milestones, or emotional 'experience-in-place' (Manzo, 2005) play a significant role in constructing meaningful people-place relationships.

Another challenge for former refugees is that it is difficult to maintain proximity to meaningful places (Hidalgo \& Hernandez, 2001) - particularly for those individuals who have resettled in a country thousands of miles from home and who face persecution in their country of origin. Fortunately, advancements in mobile communication technologies such as laptops, I-phones, and I-pads have played a significant role in compressing this distance and enabling individuals to virtually maintain meaningful relationships (Elliot \& Urry, 2010; Marlowe, 2019; McNeill, 1994). In communications researcher Ozkul's (2013) study with diverse individuals in London, a participant said that when her brother visited Montreal, he sent her a photo of a cup of coffee that made her feel as though she was with him, experiencing what he was experiencing. Thus, these siblings were able to maintain their relationship from a distance and share their unique experiences, whilst simultaneously experiencing events happening in other places. These virtual interactions are particularly important in refugee resettlement, as the internet is often the only means to see and hear distant loved ones and remain connected to home (Marlowe, 2019). However, being visually and aurally present in multiple places can also prevent

\section{Figure 2:1: 'Hoping to See} You Again'

When the first tha lei is turning, to Karen land I am longing.

I close my eyes and visualize, Karen the world over actualize.

Boothow peak at Mount K'ser

Doh, Delta Karen who are mother's folk.

Kwekabaw looks alluring and grand, beside the Salween's banks.

Pa'an town and Taw oo town, Taw Naw cliff and Naw ta Yar down.

The sun shines and the Moei River flows, under Klo Yaw cliff shade grows.

(Source: Thoo Lei Wild Grass, 2011) 
individuals from moving on with their lives and investing in their local communities as they live vicariously through others (McNeill, 1994; Ozkul, 2013).

International research also shows that former refugees and migrants commonly attempt to reconstruct aspects of place and home in their new cities, by cooking familiar foods (Hage, 1997; Law, 2001; Longhurst et al., 2009; Kale et al., 2019); planting gardens (Biglin, 2020; Coughlan \& Hermes, 2016; Rishbeth et al., 2019), and wearing traditional clothes (Arev, 2018; Lewis, 2015). Thus, in the absence of the meaningful place itself, spatial proximity is often replaced with what could be described as 'affective proximity' - the feeling of being there. The reconstruction of place is important as it shifts beyond hopeless feelings of loss and grief to question how meaningful attachments can be protected during times of disruption, whilst also enabling new placeattachments to be formed and dignity and a sense of belonging to be restored (Sampson \& Gifford, 2010).

In this manner, individuals may form and maintain attachments to multiple places simultaneously and be navigating complex (and sometimes contradictory) place-based identities and relationships (Taylor, 2013; Waite \& Cook, 2011). Feelings of place-attachment and conceptualisations of home and belonging therefore have the potential to be transnational or plurilocal, crossing the boundaries of and intimately connecting places in complex inextricable ways.

\section{Figure 2:2: 'I Do Need}

\section{Peace'}

(The first phase of my life) the village I was born far from metropolis my village alluring more than any place with mountains and forests and rivers and streams.

In the jungle growing up

I lived a barren life although my life was poor I had freedom

the beauty of Karen land my ideal location.

Without notice suffering came quick to greet us:

$$
\begin{gathered}
\text { soldiers } \\
\text { rapid }
\end{gathered}
$$

they burnt our house an annihilated place.

Rice barns to ashes our food lost inhabiting the forest deep from my house of ashes as the enemy searched for us in the basket, father took me

$$
\begin{gathered}
\text { away } \\
\text { my village }
\end{gathered}
$$

I can never see again.

The school where I fell in love

burnt down by a dictator the school's books into the ground for knowledge

I went to school in the jungle made a life in the jungle moved from place to place, day through night.

(Source: Tee Noe, 2014) 


\section{2:2 Understanding (multi)sensory experience}

\section{Sensory scholarship}

To better understand how place-attachments are formed and maintained, geographers Tuan (1977) and Seamon (1980) and environmental psychologist Lewicka (2011) have argued that there is a need to focus on processes of sensory perception. For individuals to feel emotion, to think thoughts, or to act in relation to their environment, they must first be present somewhere, physically sensing and interacting with the world and conscious of their surroundings (Damasio, 2000; Merleau-Ponty, 1962). Without explicitly addressing theories of sensory perception and consciousness it is difficult to understand how or why individuals feel, think, and act the way that they do.

As mentioned in the introduction, theories of sensory perception are grounded in human physiology and cognition and are well theorised in the disciplines of neuroscience and psychology (Baddeley et al., 2009; Barth et al., 2012; Damasio, 2000). They are also well theorised in philosophy, particularly in the field of phenomenology (which also studies the structures of experience and consciousness) (Husserl, 1989; Merleau-Ponty, 1962). In the early 1960s phenomenologist Merleau-Ponty (1962, p. xvi) challenged Descartes' famous philosophical proposition: 'cogito ergo sum; I think, therefore I am', and argued that first and foremost, "The world is what we perceive". Merleau-Ponty's claim positions the material sensing human body as central to any understanding of reality, and suggests that humans do not only exist through their cognitive awareness of themselves and the world, for this cognition would not be possible without an individual first being corporeally present somewhere and experiencing the sensory stimuli that triggers human emotions and awakens human consciousness.

Phenomenologist Merleau-Ponty's work has directly influenced human geographers, and in the late 1970s Tuan (1977, p. 6) introduced the idea that sensory perception plays a pivotal role in shaping meaningful people-place relationships. He claimed that, "What begins as undifferentiated space becomes place as we get to know it better [through our senses] and endow it with value". Building on this work, other geographers began to explore the idea of 'sensescapes' (Cosgrove, 1998), and more specifically 'soundscapes' and 'smellscapes' (Porteous, 1990; Smith, 1994). In the early 1990s, Pocock (1993) and 
Rodaway's (1994) explicit 'focusing' of the senses within geographical literature highlighted a 'sensory turn' in the discipline, which came to unfold alongside, and often intersect with, the emotional turn. This sensory turn was also noted in anthropology (Basso, 1996; Feld, 1990; Howes \& Classen, 1991; Ingold, 2000; Seremetakis, 1993; Stoller, 1997), and key contributions were made in sociology (Largey \& Watson, 1972; Low, 2005; Synnott, 1993).

Sensory scholarship has continued to expand over the past three decades, with a raft of empirical studies exploring everyday sensory practices such as doing laundry (Pink, 2005), exploring ruins (Edensor, 2007), cycling (Spinney, 2006), and eating (Sutton, 2010). These studies tend to focus on the relational affective dimensions of material bodies moving through space, engaging with dynamic stimuli, and co-constituting meaningful places through physical interaction. Meanwhile, geographers Longhurst, Johnston, and Ho (2009) and Hayes-Conroy and Hayes-Conroy (2010) have brought attention to the fact that visceral (internal) 'gut feelings' and other senses are not only spatial but inherently social and political, with specific flavours being linked to feelings of belonging, pride, or shame and local politics of inclusion and exclusion. Anthropologists Trnka, Dureau and Park (2013) have similarly focused on the politicisation of sensory embodied experience through exploring everyday feelings and practices of citizenship. These ideas are further discussed through a growing body of work on former refugees and migrants sensory experiences of homemaking, belonging, and identity (Kale, et al., 2019; Law, 2001; Longhurst et al., 2009; Munt, 2016; Tolia-Kelly, 2008).

\section{Sensory perception and Damasio's theory of consciousness}

To clarify what a process of 'sensory perception' entails and enables, I draw upon neuroscientist Damasio's (2000) theory of consciousness, before contextualising sensory perception within broader sociocultural frameworks of knowing and structuring the world. This integrated interdisciplinary approach is important as it highlights both the embodied ways in which the senses are felt - 'the feeling of what happens' (Damasio, 2000), as well as the cultural ways in which the senses are conceptualised, analysed, and valued.

Sensory perception begins with existing objects and environments (known as sensory stimuli) which each have unique visual, auditory, olfactory, gustatory, cutaneous, or other 
sensory 'qualities'. For example, a car may have the qualities of being solid, red, and at times rather loud. As humans encounter these unique sensory qualities, they are received through the body's 'sensory receptors' - defined as nerve endings that generate impulses which are processed by the brain in 'neural maps' (Baddeley et al., 2009; Damasio, 2000). These impulses lead to automatic and unconscious 'body states', or what neuroscientist Damasio (2000) sometimes refers to as 'emotions' (note that the term emotion is used differently in geography to also encapsulate conscious feelings, and that the term 'affect' is probably more salient for geographers here). Philosopher Massumi (2002, p. 60) described these affective physical body states, saying,

Walking down a dark street at night in a dangerous part of town, your lungs throw a spasm before you consciously see and can recognize as human the shadow thrown across your path. As you cross a busy noonday street, your stomach turns somersaults before you consciously hear and identify the sound of screeching brakes that careen towards you.

This awareness of one's body physically interacting with the world is defined by Damasio (2000) as an experience of the 'protoself', and comprises the first stage of consciousness (see Figure 2:3). It is this stage of consciousness which primarily concerns nonrepresentational theorists such as McCormack (2005) and Thrift (2008) and other geographers who may be exploring affective human experience.

The second stage of consciousness involves an awareness of the feelings produced by such a physical reaction. For instance, one's lungs throwing a spasm in relation to shadows in the night may evoke a feeling of fear. Being aware that one is feeling fear is to sense oneself as immersed in the present - a process which Damasio refers to as 'coreconsciousness'. These initial stages of consciousness are bound in visceral experience, or 'gut feeling'. They are also evident in non-human animals and are vital to survival.

Over time, sensory stimuli, affective body states, and associated feelings are stored in and retrieved from one's neural maps, enabling an individual to create memories which can help them to build up sociospatial reference points (to recognise people and objects and the feelings and emotions they may generate). Damasio refers to this higher level of thoughtprocessing over time as 'extended consciousness'. This third stage signifies a shift from 
affective corporeality (or an awareness of the body as being shaped in time and place) to an abstract self-representation or 'autobiographical self' which is linked to the past, present, and future and integral to the formation of identity and a sense of continuity over time. Such abstract thinking is also key to the construction of language and culture and pivotal in the ability to imagine and hope or to desire for something that has yet to materialise.

\section{Figure 2:3: Damasio's Model of Consciousness}

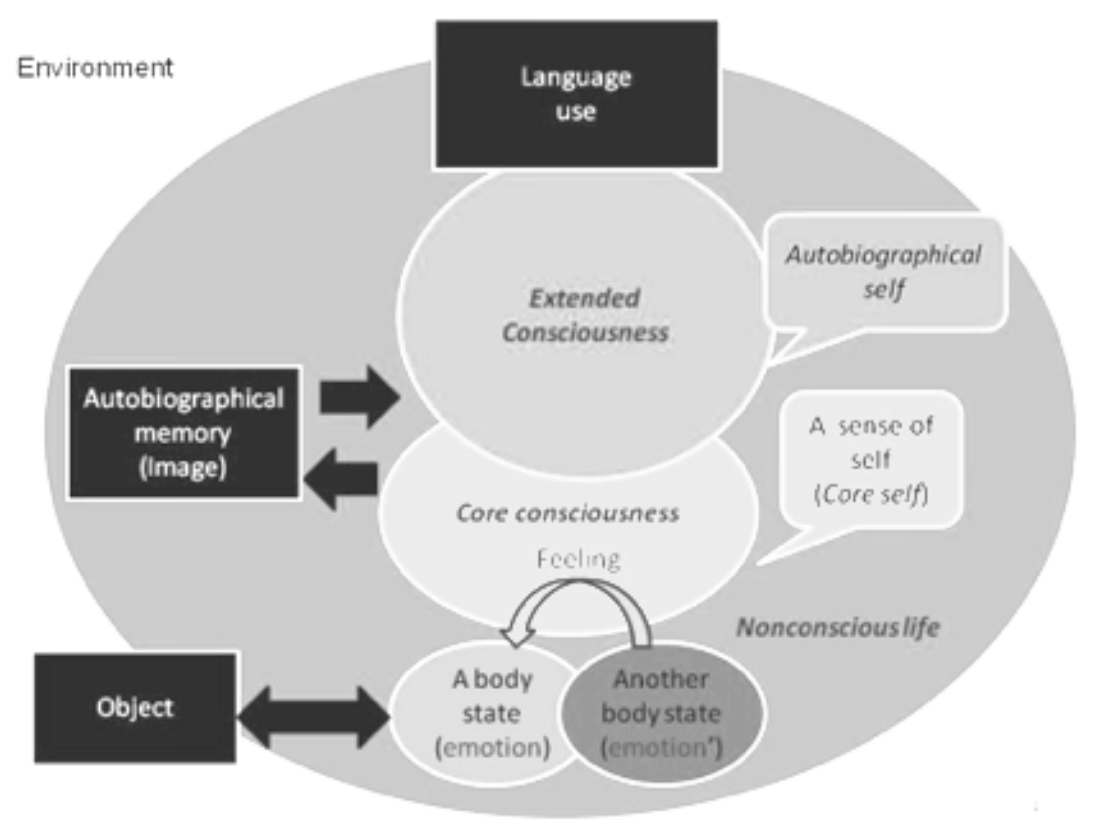

(Source: http://yosukeyanase.blogspot.com)

These sensory perceptual processes are discussed as being universal; however, an individual's lived experience will differ from others due to their unique physical characteristics and capacity for movement. Thus, someone who is blind or deaf or who cannot walk will come to know the world in different ways than someone with strong vision or hearing, or who might be capable of moving at a faster pace or covering a greater distance (Ghallagher \& Zahavi, 2008; Pocock, 1993). Different aged and sexed bodies will also interact with the world in different ways (Manzo, 2005). These nuanced experiences mean that, whilst all humans mediate the world through sensory perception, it cannot be assumed that there is one objective reality which is being perceived. 


\section{Cultural frameworks and contested ways of knowing}

In addition to the fact that bodies are different and feel differently, "the perceptual is cultural and political" (Bull et al., 2006, p. 5), and thus subject to different linguistic and structural limitations and epistemological frameworks. One way in which culture shapes sensory perceptual experience is through knowledge of what faculties or modalities constitute the senses. Many people are familiar with the Western five sense framework consisting of sight, sound, smell, taste, and touch, which has been attributed to Greek philosopher Aristotle (2015) in his 350 BC book De Caelo (On the Heavens). However, in the early 1900s, Austrian philosopher Steiner (1981) argued that there are twelve identifiable senses consisting of touch, life, movement, balance, smell, taste, sight, temperature, hearing, speech, thought, and ego (Figure 2:4).

\section{Figure 2:4: Steiner's Twelve Senses}

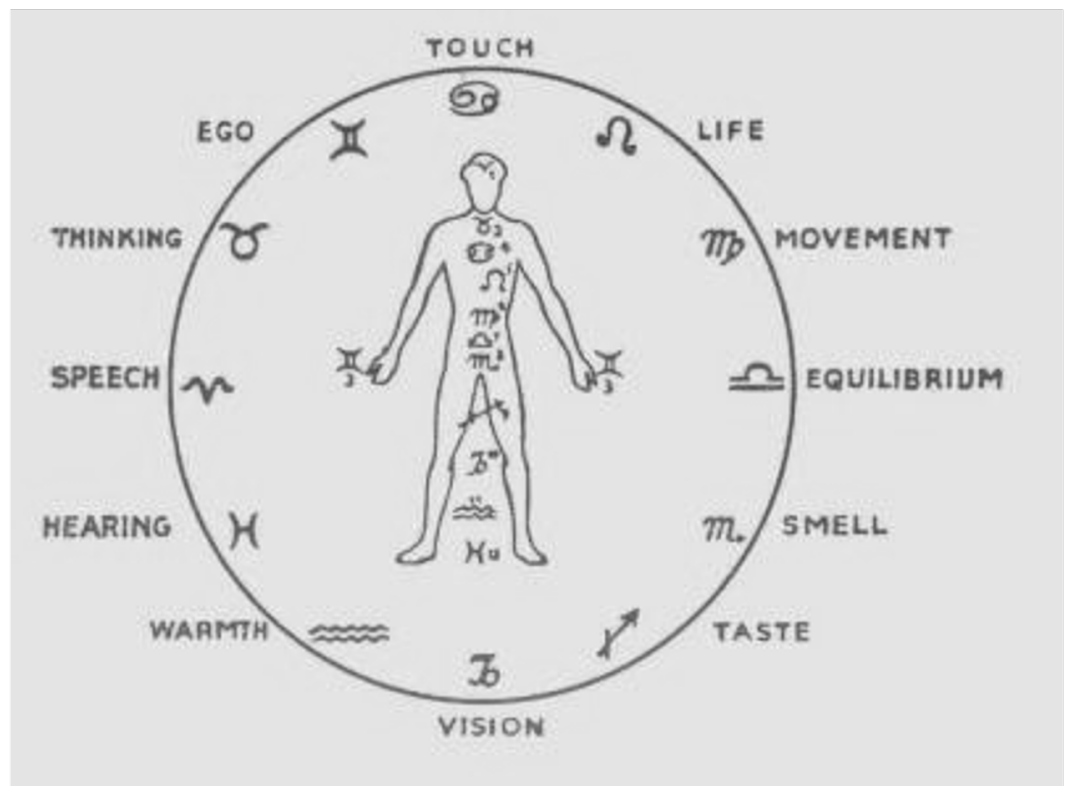

(Source: http://www.doyletics.com/arj/12signss.jpg)

Western occupational therapists have also expanded upon the five sense framework to add the vestibular (a sense of movement), proprioceptive (sensations from muscles and joints), and interoceptive (related to the physiological condition of the body) (Champagne, 2004). Meanwhile, in the East, Buddhists discuss a sixth sense pair consisting of the mind and mental objects (Bodhi, 2000), and philosopher Elberfeld (2003, p. 483) claimed that, 
In the Brhadaranyaka Upanishad [in India] a list of eight senses is given: (1) prana (breathing organ, i.e., nose; also 'breath of life'); (2) the speech organ; (3) tongue (taste); (4) eye (color); (5) ear (sounds); (6) mana (thought, mind, inner organ); (7) hands (work); and (8) skin (sense of touch).

Anthropologist Howes (2013, p. 13) said, "According to the latest scientific estimates, there are at least ten senses and possibly as many as 33". The existence of these diverse frameworks suggests that the five-sense model unwittingly obscures lived experiences and diverse ways of knowing which play an integral role in shaping individual and collective worldviews. To account for the myriad of different ways that an individual experiences the world, I draw upon these expanded sensory frameworks in this thesis. However, I accept that they too may have their limitations, and thus do not view them as exhaustive but indicative of the need to pay attention to diverse embodied ways of being and becoming.

In addition to debate over what constitutes the senses, the nature of the relationship between different senses has long been contested. In a 1907 essay (republished in 1997), sociologist Simmel advocated a separatist approach to sensory scholarship by prioritising the senses of sight and sound and labelling the remaining senses as secondary in significance to sociological study. Steiner (1981) then created three hierarchical sensory categories and claimed that the higher senses of thought and ego could transcend the physical body. This Cartesian dualistic view that the mind and body can be divided is contested in Merleau-Ponty (1962) and Damasio's (2000) work, which positions the body not as a mediator of experience but a primary source of knowledge.

Like Simmel, geographer Porteous (1990) also claimed that vision was 'overpowering'; yet, he perceived the 'alternative' modalities of sensory experience to still be of interest, and thus created 'soundscapes' and 'smellscapes' to examine these senses in more depth. Meanwhile, anthropologists Classen, Howes, and Synott (1994) argued that the senses could be distinguished, prioritised, and analysed in relation to collective ideologies and practices. This idea emerged through their comparative empirical findings that Western cultures tended to draw more heavily on visual experiences, whilst the Ongee people in the Andaman Islands paid more attention to smell. 
In contrast to these views, geographer Rodaway (1994) acknowledged the significance of multisensory perception, whereby different senses were experienced simultaneously. $\mathrm{He}$ argued that at different times and in different circumstances one or more of the senses may be heightened. Geographer Tuan (1993) and anthropologist Ingold (2000) also argued that the senses are interwoven and claimed that one ought not to be given primacy over another. These scholars are supported by psychological and neurological studies of 'synesthesia' - a perceptual phenomenon whereby the stimulation of one sensory modality inadvertently leads to experiences in other sensory modalities (Cytowic, 2003), and 'multisensory integration' - where information from multiple stimuli are integrated by the nervous system and experienced simultaneously (Stein et al., 2009).

Such integrated multisensory ways of knowing the world are evident in Burmese former refugee student Kang Pu's (2016) poem, 'My Mother's Kitchen' (Figure 2:5). This narrative intertwines multiple senses and draws upon memories of different times and places to portray an atmosphere that is unique to Kang Pu's mother's kitchen in Myanmar.

The reader would surely be deprived of knowing this place as Kang Pu did should they only be informed of the sights or smells and not attuned to the whole multisensory experience. Drawing upon these theories, I work from the premise that the world is multisensory and humans are physically subjected to multiple senses simultaneously. Yet, due to unique sociocultural and personal histories, not everyone will analytically interpret the senses as interwoven or remember and describe them the same way.

\section{2:3 The role of the senses in everyday wellbeing}

\section{Traumatic and nostalgic sensory memories}

Through the ability to evoke affective feelings and emotions, the senses have particular relevance in studies of place-attachment and wellbeing. Interaction with

\section{Figure 2:5: 'My}

Mother's Kitchen'

When my mom cooked it smelled of sweet wintertime cherries, of a solitary forest with rain falling and it smelled like the murmur of a lonely bird singing.

I picture the spherical smoke rising from her kitchen

it was like the sound of sleep at night, it was like arriving home safe and sound the sounds of her kitchen were peaceful.

(Source: Kang Pu, 2016) 
environmental stimuli can lead to surprise, joy, happiness, fear, and disgust, shaping the emotional place bonds discussed above by Tuan (1974), Relph (1976), and Manzo (2005). Memories are also triggered by familiar stimuli (Baddeley, 2009; Damasio, 2000) and may elicit strong visceral affective and emotional responses which negatively or positively influence how an individual relates to their environment (Drozdzewski et al., 2016; Hadi Curti, 2008; Hinton et al., 2004; Kidron, 2012; Tolia-Kelly, 2008).

In particular, the negative impact of sensory memory on refugee wellbeing has been well documented through a focus on Post Traumatic Stress Disorder (PTSD) (Hinton et al., 2004; Hinton et al., 2011). In psychiatrist Hinton et al.,'s (2004, p. 164-165) study of olfactory-triggered panic attacks among Khmer refugees in the USA, one of the case studies stated that,

Upon arrival in the United States, Meng noticed that exposure to car fumes often triggered dizziness, palpitations, and other symptoms ('a wind attack' cay $\boldsymbol{k a \partial t}^{\boldsymbol{h}} \boldsymbol{y a l}$ ). These panic attacks lasted about 10 minutes. Initially, Meng did not understand the reason for the reactivity. Through clinical interviews we learned that after the Vietnamese invasion of Cambodia, Meng had tried to escape to Thailand but had been caught by the incoming Vietnamese soldiers. The soldiers forced her to squat down with her head positioned just below the foot-wide barrel of a mortar-shooting cannon. Then they fired the gun, which blasted sulfurous fumes and caused a severe ringing in Meng's ears. She fainted.

Even without a diagnosis of PTSD individuals may feel uncomfortable in sensory environments which trigger unpleasant memories (Tolia-Kelly, 2008). This ambivalence of the past and present may leave individuals feeling disorientated and uncomfortable, and prevent them from feeling safe in their current environment.

Yet, sensory memories can also have a positive impact, helping displaced individuals to maintain connections to their former homes and continue a sense of identity or transnational belonging through feelings of 'nostalgia' (Blunt, 2003; Rishbeth \& Powell, 2013; Tolia-Kelly, 2008). Geographer Ratnam (2018, p. 9) claimed that,

Our senses — touching, tasting, smelling, seeing, and hearing — link to familiarity and security of home(lands) and also the sensorial experiences of home cooking and the material that provide comfort. Such experiences build on a homely sense of 
community and belonging through recollection and remembrance. These, in turn, contribute to our individual and collective identities and vice versa.

Nostalgia has not always been synonymous with positive memories, nor has it always been associated with embodied experience. Deriving from the Greek 'nostos' - which means 'return home' - and 'algos' - which means 'pain' - nostalgia originally denoted a sense of homesickness and longing for a distant homeland. In the $17^{\text {th }}$ to $20^{\text {th }}$ century, such homesickness was considered to have a profound effect on the body and mind to the point where it could result in physical illness (Blunt, 2003; Morse \& Mudgett, 2017).

In European and North American literature in the late 1980s and early 2000s, these physical conceptualisations shifted to cognitive understandings of nostalgia as a 'state of mind' (Morse \& Mudgett, 2017; Rubenstein, 2001). Feminist literary theorist Rubenstein (2001, p. 4) claimed that,

Nostalgia encompasses something more than a yearning for literal places or actual individuals. Whilst homesickness refers to a spatial/geographical separation, nostalgia more accurately refers to a temporal one. Even if one is able to return to the literal edifice where $\mathrm{s}$ /he grew up, one can never truly return to the original home of childhood, since it exists mostly as a place in the imagination.

Through this delineation of the spatial (and perceptual), Literary critic Stewart (1993, p. 23) claimed that, "Nostalgia is a sadness without an object, a sadness which creates a longing that of necessity is inauthentic because it does not take part in lived experience". She therefore romanticised nostalgia as "the desire for desire", or a desire for a particular kind of feeling rather than a tangible place. Because places are always changing, geographer Massey (2005, p. 65) also claimed that the feeling of loss that often accompanies spatial change is "a nostalgia for something that did not exist".

However, other researchers have challenged these views and explicitly aimed to respatialise nostalgia and relocate it in embodied multisensory practice. In her study of memory with Anglo-Indians at McCluskieganj, geographer Blunt (2003, p. 722) used the term productive nostalgia to, "Represent a longing for home that was embodied and enacted in practice rather than solely in narrative or imagination". Through enacting familiar traditions from home in a new place, participants in her study were deconstructing 
the notion of nostalgia as 'psychologically regressive' or pathological, and demonstrating the positive 'liberating' effect it could have in enabling them to express themselves and construct home in new places (Rubenstien, 2001).

Former refugee participants in sociologist Biglin's (2020) research in the UK Northwest and sociologists Coughlan and Hermes (2016) research in New York also enacted nostalgia by recreating gardens, and in geographer Law's (2001) Hong Kong research and anthropologist Hage's (1997) research in Sydney, migrants recreated familiar sights, sounds, smells and tastes through cooking traditional cuisine. In my research with former refugees and local residents in Wellington, participants also talked about recreating feelings of homeliness through the making and drinking of specific types of coffee (Kale et al., 2019). Meanwhile other former refugees were enacting the past in the present through creating textiles (Dudley, 2010) and wearing traditional clothing (Arev, 2018; Lewis, 2015; Rishbeth et al., 2019).

Unfortunately, the enactment of nostalgia and introduction of 'foreign' cultural stimuli can lead to social tensions between newcomers and long-term residents (Hage, 1997; Kale et al., 2019; Law, 2001). Sociologist Simmel (1997 [1907]) argued that an individual's sensory impression of other people shapes their emotions and knowledge of them, and sociologists Low (2005), and Largey and Watson (1972), claimed that smell is used to differentiate people and shape social boundaries between 'us' and 'them'.

To enhance an appreciation for multisensory diversity, Longhurst, Johnston, and Ho's (2009) research with migrant women in Hamilton discussed the significance of sharing food to enhance embodied social connections. Participants in my Masters' research (Kale et al., 2019) also suggested investing in more public places like multicultural markets, where individuals can experience different sounding music, different smelling and tasting cuisines, and unique tactile crafts and, through these shared experiences, become similarly situated and more empathically attuned to others. These collective multisensory activities are particularly important in terms of enhancing place-attachment and wellbeing as they can help former refugees to feel included, valued, and welcome in places of settlement, and to feel as though they belong somewhere. 


\section{Feelings of (dis)comfort}

Everyday multisensory stimuli can also impact how (un)comfortable an individual feels from moment-to-moment and alter their levels of stress and anxiety. In human ecologist Evans' (2006) study on the role of the physical environment in childhood development, he discussed the negative impact that loud noises, crowded spaces, mouldy buildings, bright unnatural lighting, and uncomfortable temperatures can have upon a child's wellbeing and their ability to concentrate and learn. He claimed that these environmental conditions can also negatively impact adults, making them feel stressed, tired, and less capable of providing the attention that young children need to thrive.

Psychologists Saxbe and Repetti (2010, p. 79) also highlighted the correlation between uncomfortable stimuli and a poor sense of wellbeing in their linguistic analysis of the link between clutter in the home and levels of stress in married working women. They claimed that, "Women who see their homes as a source of demands (the need to straighten up clutter or complete unfinished projects) might have more difficulty unwinding effectively from the workday. In other words, feelings about the home might moderate everyday adaptation to the environment".

To relax and escape the pressures of loud, bright, chaotic places or sensory 'triggers', individuals often seek out environments in which stressful stimuli are minimised (Kaplan \& Kaplan, 1989). In geography, these places have been referred to as 'therapeutic landscapes'. Whilst geographer Gesler (1992; 1996), initially emphasised the physical, mental, and spiritual healing properties that iconic places like public baths and pilgrimage sites held for different people, other researchers have challenged assumptions that places may be intrinsically therapeutic, and argued that the subjective and changing nature of individual lived experience demonstrates therapeutic value as being relationally constructed (Conradson, 2005; Kearns \& Collins, 2000; Williams, 1999).

Geographer Wilson (2003) also challenged the focus on 'extra-ordinary' sites of healing, and argued that therapeutic landscapes was a Western conceptualisation which excluded other alternative ways of linking health and place. In her research with First Nation Peoples in North America, she claimed that there was a need to move beyond the extraordinary to focus on everyday culturally specific practices of healing and wellbeing. 
Researchers have since explored the therapeutic value of ordinary multisensory landscapes such as beaches, rivers, parks, and gardens (Bell et al., 2015; Collins \& Kearns, 2007; Milligan \& Bingley, 2007), describing these environments in visual terms as 'green spaces' (Bell et al., 2018; Milligan \& Bingley, 2007) or 'blue spaces' (Bell et al., 2015; Collins \& Kearns, 2007; Volker \& Kistemann, 2011).

Geographers Wolch, Byrne, and Newell (2014, p. 234) claimed that,

Public green space includes parks and reserves, sporting fields, riparian areas like stream and river banks, greenways and trails, community gardens, street trees, and nature conservation areas, as well as less conventional spaces such as green walls, green alleyways, and cemeteries ... Private green space includes private backyards, communal grounds of apartment buildings, and corporate campuses.

Meanwhile, geographers Foley and Kistemann (2015, p. 158) stated that, "Blue space is defined as; 'health-enabling places and spaces, where water is at the centre of a range of environments with identifiable potential for the promotion of human wellbeing'”.

The focus on the health benefits of outdoor landscapes links into an earlier (still expanding) body of work in environmental psychology. Studies have shown that patients in hospitals who were privy to views of nature outside their windows were rehabilitated faster (Ulrich, 1984; Verderber, 1986); and inmates in prison who had views of nature sought health care less often than inmates who did not have these views (Moore, 1981; West 1986). Interacting in green spaces made people feel healthier (Godbey et al., 1992; Maas et al., 2006), and in a project with park visitors in Amsterdam, Paris, and Seville, environmental researcher Chiesura (2004, p. 338) found that urban parks are important for residents, "To relax and step away from the hectic rhythm of the city", as well as being important places to spend time with their children and socialise. Similarly, in their study with Swedish university students, environmental psychologists Hartig and Staats (2006, p. 223) claimed that, "Our participants had a more positive attitude toward walking in the forest than walking in the city, and this difference was larger among the more fatigued participants".

The health benefits of therapeutic green spaces have also been explored in refugee resettlement, most notably in the UK, USA and Australia through practices of gardening 
(Biglin, 2020; Coughlan \& Hermes, 2016; Rishbeth et al., 2019; Sampson \& Gifford, 2010). These studies found multiple ways in which gardens could be therapeutic, from the aforementioned re-connecting of individuals with nostalgic memories of home, to enhancing fresh air and fostering new connections with the land, as well as enhancing sociability.

However, the focus on green and blue landscapes has been critiqued for being romanticised and obscuring potential risks such as attacks by other humans or animals (Gorman, 2017; Milligan \& Bingley, 2007), or accidents such as drowning (Collins \& Kearnes, 2007). In their study of refugee experiences in Berlin, London, and Shieffield, geographers Rishbeth, Blachnicka-Ciacek, and Darling (2019) claimed that women may also have heightened fears in public recreation places due to gendered issues of harassment and violence. Meanwhile, geographer Brown (2016) claimed that it is important to move beyond the visual colour-coding of green and blue landscapes and explore the texture of the land. Geographer Gorman (2017) also suggested moving beyond the visual to consider the role of non-human entities in co-constituting a therapeutic (or non-therapeutic) experience.

Whilst there has been a disproportionate focus on the outdoors, geographers Dyck and Dossa (2006) have explored the home as a therapeutic place for former refugee women in Canada, and refugee-background participants in resettlement researchers Sampson and Gifford's (2010, p. 116-117) Melbourne study talked about feeling safe, happy, and healthy in their bedrooms, classrooms, and libraries. The authors claimed that, "These places, conceptualized together as therapeutic landscapes, are critical for facilitating positive connections to place, promoting well-being and contributing to new arrivals' becoming at home in their country of resettlement".

Other studies have focused on the health benefits of spiritual places (Agyekum \& Newbold, 2016; Foley, 2013; Gesler, 1996; Maddrell, 2013); however, sites of worship and pilgrimage have been prioritised over everyday personal religious practices (Dyck \& Dossa, 2006; Wilson, 2003). The cognitive benefits of religion have also been prioritised over physical or emotional experience in the social sciences (McGuire, 2016; Plate, 2012). Sociologist McGuire (2016, p. 152) claimed that, 
Our discipline has traditionally focused on religion's cognitive qualities - beliefs, creeds, and theologies - along with its organisational structures. By and large, we have failed to encounter, much less think about, other aspects of religion, particularly those that involve the senses.

Whilst the term 'therapeutic landscapes' remains common, the fact that places are not intrinsically therapeutic, and not only comprised of land (but also sea, sky, people, and other non-human entities) has brought this terminology into dispute. Recent research has instead referred to 'healthy places' (Bell et al., 2018), and 'therapeutic assemblages' (Gorman, 2017), amongst other variations. To draw out the multisensory, emotional experiences that comprise a therapeutic encounter (be it with a natural, built, social, or more-than-human entity), I henceforth refer to 'therapeutic-feeling places' in this research. In retaining the word therapeutic, I focus on both processes of healing to relieve pain, fatigue, worry, and stress, and processes of relaxation or rejuvenation which aim to enhance contentment and prevent fatigue or stress from occurring.

\section{Being well and feeling well in place}

The expanding body of research on therapeutic landscapes signifies an important shift in how geographers conceptualise and study mental health and wellbeing. Mental health geographies first emerged as a sub-discipline in the early 1970s, with research exploring how individuals with psychological disorders accessed and utilised different places; and how the physical and social environment affected individual emotions, thoughts, and behaviours (Jones, 2001; McGeachen \& Philo, 2017; Philo \& Wolch, 2001). This work predominantly focused on pathological aspects of mental health, focusing on individuals who were diagnosed with serious conditions and considered by society to be 'mad', 'deviant', 'different', 'disabled' or 'abnormal' (Philo \& Wolch, 2001).

It wasn't until around the turn of the century that researchers began to pay attention to mental health in the wider human population (including those without known mental illnesses), and to consider the role of everyday environmental stressors in feelings of wellness. Geographers Kearns and Andrews (2010, p. 99) claimed that recent studies have involved, "Common yet often neglected and trivialised psychological problems that might not be categorised strictly as mental illnesses, yet nevertheless impact significantly upon 
people and their wellbeing". As mentioned in Chapter One, they described wellbeing as ( $\mathrm{p}$. 98), "Both being well (i.e., having access to life's material necessities) and feeling well (having the capacity to act and find meaning in the world)".

Discussing the English etymology of the word 'well' (in wel-come and well-being), language professor Phipps (2019a) claimed that well referred to a spring of water with miraculous origin or healing powers, and linked to ideas of wealth and will. In this sense, wellbeing or being well may also encompass notions of healing and recovery, happiness and prosperity, and desire or intent.

With this shift to everyday wellbeing, geographers started to focus on positive experiences of wellness and feelings of contentment and happiness. Pain and Smith (2010, p. 89) claimed that, "For a while, it seemed complacent to think about the opposite end of the spectrum: to explore the condition of contentment, the state of wellness, or the predictors of wellbeing. But all this has changed in recent years". They stress that this shift is important because addressing mental health issues does not necessarily lead to strong resilient happy individuals, although it is an important step towards feeling well.

These approaches resemble the holistic Māori models of wellbeing, Te Whare Tapa Whā (Durie, 1985) - which I expand upon in the following chapter, and Te Wheke (Pere, 1997). They also resemble the 'medicine wheel' of the Anishinabek people in North America (Wilson, 2003). Such models recognise that the physical, emotional, mental, social, and spiritual aspects of one's life require balance and care to prevent individuals from feeling unwell. They encompass a dynamic relational understanding of wellbeing as emerging through individual interactions with physical, natural, and built environments - an ontology which has become central to the work of geographer Atkinson (2013) who suggested that wellbeing should not be understood as an outcome, but a process.

Atkinson et al. (2012, p. 3) also argued that,

Wellbeing, however defined, can have no form, expression or enhancement without consideration of place. The processes of well-being or becoming, whether of enjoying a balance of positive over negative affects, of fulfilling potential and expressing autonomy or of mobilizing a range of material, social and psychological resources, are essentially and necessarily emergent in place. 
The concepts of wellbeing and place-attachment are closely interlinked. Wellbeing can enhance feelings of place-attachment through enabling individuals to feel safe, happy and healthy in place and to form positive emotional associations with their surroundings. Meanwhile, place-attachment can enhance wellbeing through increased social bonds, trust, self-esteem, and feelings of belonging, coherence, and meaning (Lewicka, 2011; Scannell \& Gifford, 2016). Conversely, one does not need to feel well to feel attached to a place, and one does not need to feel attached to place to feel well. These concepts should therefore not be considered as mutually dependent, but rather complimentary. Because of its extensive reach into various aspects of everyday life, there is an extensive body of interdisciplinary research on wellbeing. Focusing on multisensory affective and emotional experience and geographic research only scratches the surface of this literature; however, this tailored approach is important in centralising the connection between place and health.

\section{2:4 Key learnings}

This literature review has highlighted the need to further explore place beyond its social networks and to consider how people form attachments to physical environments, such as bedrooms, libraries, mountains, rivers, coastlines, flora, fauna, and seasonal cycles and weather patterns. There is also a need to explore place as multisensory, comprised of a diverse array of visual, auditory, olfactory, gustatory, cutaneous, and other sensory qualities which can affect human bodies in unique ways and shape individual emotions, thoughts, and behaviours.

Sensory geographies are not new, and (multi)sensory experience has been intermittently explored throughout theories of place-attachment for more than 50 years. However, this focus becomes particularly salient in light of the shift toward a relational approach to understanding lived experience over the past two decades. Rather than discussing feelings, emotions, and thoughts as though they are independently generated and confined to the subjective self, studying the senses can enable researchers to directly link external phenomena with embodied feeling. This is important as multisensory experience often blurs the borders of self and other, or 'inside' and 'outside', particularly in visceral 
experiences such as eating, whereby an external object may permeate the body with unique smells and flavours (Hayes-Conroy \& Hayes-Conroy, 2010; Longhurst et al., 2009).

Understanding these dynamic relationships between individuals and their multisensory surroundings can enhance understanding of how place-attachments are formed, negotiated, and maintained. It can also enhance understandings of how everyday interactions with place might influence negative or positive emotional associations and memories and affect an individual's sense of holistic wellbeing. In this manner, being well encompasses affective neurophysiological feelings of wellness, as well as a broader sense of identity and belonging that can enable individuals to feel secure in place and give their lives meaning.

A focus on the senses also enables insight into how emotional experience shapes place. As Blunt (2003) claimed, emotions such as nostalgia can be productive and transformative as individuals recreate multisensory aspects of their former homes and imbue new places with meaning. This in turn influences how an individual feels in place, as well as how others feel. Such dynamic relational processes require a dynamic relational framework in order to be understood, and I develop this framework in the following chapter.

Bringing this discussion back to the context of refugee resettlement, it is important to reiterate that there are multiple places to which an individual may become attached during their lifetime, and these attachments can occur concurrently, enabling individuals to develop and maintain meaningful relationships with both proximate and distant homes. Whilst researchers need to acknowledge the difficulties that may result through separation from meaningful places, it is also important to realise that building attachments in new places offers an opportunity for those who have been displaced to once again work the land and fall in love, and to create new memories and legacies that will live on through the generations to come. 


\section{Chapter 3: An Emplaced Conceptual Framework}

To guide my research practice and analyse participants' multisensory experiences of placeattachment and wellbeing, I develop an 'emplaced' conceptual framework in this chapter which situates individual experience within wider sociospatial and temporal contexts. Beginning with the human-centric theories of phenomenology and embodiment, I trace a historical trajectory of geographic thought toward a relational ontology, whereby individuals are intimately connected to other people, non-human entities, and distant places through a myriad of dynamic interactions, networks, and flows. This relational focus is important as it enables an exploration of the unique histories, experiences, and routines of different places, as well as the wider global events that influence local development and place-based experiences. However, thinking relationally has also raised debates over geographic notions of scale and territory, key points which I expand upon in Section 3:2. I then conclude by introducing a practical relational model of emplacement, through which the research findings are later interpreted and understood.

\section{3:1 Contextualising human experience}

\section{From embodiment to emplacement}

As mentioned in the previous chapter, early human geographers such as Buttimer (1980), Relph (1976), Seamon (1980), and Tuan (1977) were strongly influenced by the phenomenological proposition that human experience begins with the sensing feeling material body. Phenomenologist Merleau-Ponty (1962) had argued that there was a need to focus on how people physically interact with and become aware of the world, as opposed to relying on psychological analyses of human experience which create ambiguity and dilute 'facticity' or factuality. To achieve this, he advocated for an embodied approach to social science research which stepped back from abstract theorising to first explore the tangible objects and events which influence individual perceptions, feelings, thoughts, and behaviours. 
Embodiment gained traction in geography in the 1990s, becoming a central strand of feminist, critical race, and queer theory discourse. Drawing on gender theorist Butler's (1988) ideas of 'performativity', and geographer Nelson's (1999) call to spatially contextualise embodied performance, research explored how social identities were physically and discursively enacted through the spatiotemporally-situated self (Longhurst \& Johnston, 2014). Embodiment also became central to the work of geographers Thrift (1996) and McCormack (2005) in their development of 'Non-Representational Theory' (NRT), which aimed to move beyond social representations and imaginings to focus on performance and production - the 'doing' and 'creating' of experience rather than the reflecting and analysing or predicting of events. Thrift (2008, p. 113) claimed that, "NonRepresentational Theory is an approach to understanding the world in terms of effectivity rather than representation; not the what but the how". This question of how experience happens is, for supporters of NRT, grounded in the pre-cognitive concept of affect and located between bodies and other stimulating material entities.

Because Thrift (2008) and McCormack (2005) perceived affect to be a relational process emerging through an individual's external interactions, they began to critique an embodied humanistic approach, acknowledging that there are wider social and environmental influences that shape human sensory and perceptual experience, and that these influences remain obscured when the subjective self is centralised. This led to a geographic shift from embodiment to emplacement, and the idea that, "To be is to be in place" (Casey, 2009, p. 14). Seamon (2018, p. 2) claimed that, "We are always emplaced in that we always already find ourselves present to some world, whether that world is relatively temporary (attending a meeting in an unfamiliar city) or permanent (tending a family farm one's entire life)".

The concept of emplacement has also emerged in other disciplines. Philosopher Malpas (2001, p. 231) added that,

What we are as living, thinking, experiencing beings is inseparable from the places in which we live - our lives are saturated by the places, and by the things and other persons intertwined with those places, through which we move, in which our actions are located, and with respect to which we orient and locate ourselves. 
Meanwhile, anthropologist Pink (2015, p. 28) claimed that emplacement, "Attends to the question of [human] experience by accounting for the relationship between bodies, minds, and the materiality and sensoriality of the environment". In this manner, she claimed that emplacement has replaced the notion of embodiment and offers a particularly salient approach to theorising and doing sensory research.

\section{The influence of society and non-human entities}

In an effort to decentralise and re-emplace the subjective self, social scientists have focused their attention on intersubjective experience and the influence of social systems and structures. Individuals can be affected by other people producing sights, sounds, and smells (Hage, 1997; Law, 2001; Wise, 2005), or through the nurturing embrace of a loved one or a violent physical assault (Classen, 2012). People may also affect others through simple environmental actions such as turning on lights, leaving clutter, or playing music (Evans, 2006; Saxbe \& Repetti, 2010); or they may implement more substantial longlasting structural changes. Geographers Smith and O'Keefe (1996) and Harvey (1996) argued that capitalist modes of production (including agricultural practices, urban development, and industrialisation) have shaped rural and urban experiences by limiting wilderness and changing the climate, to altering the texture of the terrain and how people live out their daily lives. These changes can evoke positive or negative emotions and memories and affect peoples' health and how they feel toward a place.

Sociospatial experience is also intersubjectively shaped by language, as people construct a shared reality or collective ontological worldview with others. Geographer Tuan (1991) claimed that language is central in communicating social ideas and plans for how space should be used and adapted, and for whom and what purpose. He says that language has 'metaphorical power' through the way it is used to represent the world and make specific elements visible. Thus, everyday language use through casual conversations, political rhetoric, texts, films, media, and social media impact how one's environment is perceived, valued, and lived in, and shape what is considered 'appropriate' or 'normal' behaviour within a specific locale. However, embodied affective experiences also continue to shape human intellect and cultural norms as individuals challenge social worldviews that don't align with their own unique perception of reality. 
Equally influential are social constructions of territory, citizenship, and belonging. Citizenship or residency status, and an individual's legitimacy in the eyes of other residents and citizens, can enhance or restrict access to places, systems, and resources (Askins, 2016; Kale et al., 2020; Staeheli, 2010). Social identities of race, class, gender, sexuality, and religion further impact where individuals can or cannot travel, how they express themselves in public, and how they are treated by others. Thus, as geographer Conradson (2005, p. 340) claimed, the self is generally understood "not as an autonomous, tightly bounded entity but rather as something that emerges within and through its relations to other people and events". Anthropologists Howes and Classen (2014) and Valli (2015) therefore argued that an inter-subjective framework is needed which shifts from phenomenology to draw upon social and cultural theory.

Places are also comprised of non-human entities; the recognition of which has recently led to a flourishing body of research on 'more-than-human worlds' (Abram, 1997; Bawaka Country et al., 2016; Bridge \& Smith, 2003; Gorman, 2017; Larsen \& Johnson, 2016; Whatmore, 2002). As with NRT and social theories, more-than-human theories challenge phenomenology and embodiment for being too localised and partial, and disregard "any vantage point that purports to take in the world at a glance" (Whatmore, 2002, p. 7). Focusing on human interactions with commodities, geographers Bridge and Smith (2003, p. 258) claimed that, "The production, exchange, and consumption of commodities are constitutive of social relations ... Commodities 'make a difference' to the way social processes play out". Geographer Swanton (2016) also claimed that material objects and atmospheres, including buildings, clothing styles, food, music, language, and smells can shape social relations and play into the enactment of race and racial difference, and geographers Phillips, Evans, and Muirhead (2015) claimed that ordinary everyday objects have the potential to inspire curiosity and enchantment.

Geographic terrain and wildlife also play a role in the production of social life. Mountains may provide protection from advancing warfare, fertile soil can enable a rise in agricultural practices, and access to rivers and oceans creates unique opportunities for trade (Marshall, 2015). Meanwhile, animals bite, evoke allergies and phobias, provide emotional support, and have the potential to reduce loneliness and anxiety (Gorman, 2017; Milligan \& Bingley, 2007). Wildlife can also shape cultural norms and traditions (Bawaka Country et 
al., 2016; Mirante, 2006). In a 2006 article published online by Guernica Magazine, Mirante (the director of an information-sharing project in Myanmar) wrote about the brass neck rings worn by girls and women in Myanmar's Lahwi Kayan tribe. Whilst there are multiple reasons as to why these rings may be worn, she claimed that,

Some claim that the practice was meant to protect women from tigers, which are known to seize their prey by the scruff of the neck. In any case, the custom grew to have strong implications of power rather than helplessness, and the rings were an aspect of one of the modern world's few matriarchal cultures. While the neck-rings and similar coils on arms and legs hampered mobility, the men of the Kayan tribe did help out with childcare and everyday drudgery, and value the opinions of those who wore the weight of metal.

Thus, a fear of wild animals and the innovative use of objects like metal coils influenced how women physically moved their bodies and engaged with their surroundings, and the way that cultural gender roles and social power dynamics were enacted. In this manner, geographer Whatmore (2006, p. 602) claimed that a more-than-human focus, "Shifts the register of materiality from the indifferent stuff of a world 'out there', articulated through notions of 'land', 'nature' or 'environment', to the intimate fabric of corporeality that includes and redistributes the 'in here' of human being".

\section{Tensions of agency}

The idea that non-human entities influence human experience is not new. Geographers Larsen and Johnson (2016, p. 151) claimed that,

As the geographical self has been defined, places require human agents. Without human agency, places do not exist (Casey 2001, 684). Many Indigenous ontologies, though, hold a contradictory position. Not only does "place come first," as Aboriginal Australian author Tim Winton put it (Reimer 2008), but place has agency independent of human embodiment or awareness and, in fact, human embodiment and awareness are an extension of the agency of place.

New Zealand's Indigenous Māori and Myanmar's Chin community also expressed these animist beliefs, acknowledging that each independent entity in the world (including the weather, oceans, rivers, mountains, flora, fauna, and humans) exhibited its own unique life 
force and contributed to the nature of a place and the events that occurred there (Harvey, 2005; Smith, 1994). However, largescale British colonisation of Māori and Chin people and their conversion to Christianity during the 1800 s, and a steady rise in atheism in New Zealand (Stats NZ, 2019a), may have shifted these traditional understandings of place as autonomous.

The agency of place is also evident in Western philosophy. In the early middle-ages German philosopher Magnus argued that people were connected to the places where they were born as their environment shaped their physiological characteristics and capabilities. This argument formed part of a wider body of literature on environmental determinism, whereby physical environments were understood to shape unique human traits (Cresswell, 2013). Biologist Darwin's (1859) theory of evolution supported Magnus' ideas as he argued that natural evolutionary processes lead to unique place-based traits which can help various life forms to adapt to specific locales to enhance their chance of survival. However, Darwin adopted an absolute deterministic view, which diverged from Magnus' suggestion that humans also have a degree of 'free will' and can manipulate their environment (Cresswell, 2013).

These tensions between environmental determinism and free will have evolved to twentyfirst century geographical debates on place agency versus human agency, centralising the question, to what extent do environments shape people, and to what extent do people shape environments? (Ash \& Simpson, 2016; Larsen \& Johnson, 2016; McCormack, 2017). Such questions are important to consider in studies of place-attachment and wellbeing, as whilst other people and more-than-human entities can influence how an individual feels, human agency indicates that one can also control their environment and influence others to some degree, and therefore potentially moderate one's responses to external stimuli, the feelings evoked, and the generation of a sense of wellbeing.

Building upon the notion of more-than-human worlds and place-agency, geographers McCormack (2017) and Ash and Simpson (2016, p. 63) have advocated for a postphenomenological approach to understanding human experience, which focuses on the "excessive world that lies outside of the human-environment correlate but which is central to shaping human capacities, relations and experiences". This extension beyond the human 
body is important in enabling a greater understanding of how individual lived experience is linked to broader phenomena within a wider socio-ecological system. Shifting from embodiment to emplacement makes space for these diverse spatial entities and relationships to be articulated and understood. However, individual sensory and emotional experience is not only shaped by proximate entities and relationships, but also by distant events and places (Amin, 2004; Marston et al., 2005; Massey, 2005). Relationships are also dynamic (Amin, 2004; Thrift, 2008), constantly changing and altering place and raising the question as to where and when an experience of 'emplacement' might extend. This question is teased out in the discussion below.

\section{3:2 Thinking relationally}

\section{Contesting spatial boundaries}

For many English speakers, the use of the term 'emplacement' will likely evoke an image of an object (often heavy artillery) entrenched or carefully positioned in place. There is a sense of rootedness and stagnation connected to this image, an inherent feeling of immobility and resistance, and an implied territorial defensiveness - the idea that the subject of emplacement rightfully belongs to this location and ought not be disrupted nor imposed upon. In this sense, emplacement accords with earlier humanistic geographers understandings of place as static and bounded (Buttimer, 1980; Casey, 1996; Tuan, 1974).

However, in recent years these stagnant well-defined understandings of being-in-place have been challenged by post-structural theorists who argue that place is relationally constructed via dynamic internal and external relationships, networks, and flows. Geographer Cresswell (2013, p. 218) said, "Rather than thinking about the inhabited world as a set of discrete things with their own essences (this place, different from that place), we can think about the world as formed through the ways in which things relate to each other". Thus, the entities, activities, and rhythms of a place are not operating in a closed circuit, and cannot be taken-for-granted, but are constantly shaping and being shaped by other external forces (Amin, 2004; Cresswell, 1996; Massey, 2006; Sibley, 1981). The idea that places are closely connected to and shaped by other places is particularly salient in the 
context of refugee resettlement as people migrate between places, bringing with them new cultures, ideas, practices, and objects that can influence people and places in various ways.

This shift to thinking relationally has posed challenges to the hierarchical and concentric notion of geographic scale, whereby local, national, and global places have been talked about as distinct entities with varying degrees of influence, power, and political importance. In 2005, geographers Marston, Jones and Woodward suggested that retaining a focus on scale played into notions of place as 'fixed' 'predetermined' and 'essentialist'. Instead, they proposed a flat ontology which completely does away with scale to focus on spatial mobility and openness. Thus, different sites of spatial activity and meaning are connected to and influential of one another, yet there is no overarching political framework that divides and governs space. They describe this ontological shift as a process of deterritorialisation.

The suggestion that scale and territory are obsolete in a fast-paced interconnected modern world has been strongly contested by other human geographers, political scientists, and place-attachment theorists. For instance, geographer Casey (1997, p. xiii) claimed that, Certain devastating phenomena of this century bring with them, by aftershock as it were, a revitalized sensitivity to place. Precisely in its capacity to eliminate all perceptible places from a given region, a prospect of nuclear annihilation heightens awareness of the unreplaceability of these places, their singular configuration and unrepeatable history.

Meanwhile geographer Paasi (2004, p. 542) said,

Scales are not fixed, separate levels of the social world but, like regions/places, are structured and institutionalized in complex ways in de/reterritorializing practices and discourses that may be partly concrete, powerful and bounded, but also partly unbounded, vague or invisible. Scales are also historically contingent; they are produced, exist and may be destroyed or transformed in social and political practices and struggles.

Political scientist Kogl $(2008$, p. 143) also argued that scale and place retain their significance as, "The human body is always local, living a particular life in a particular place, with others, for better or worse”. Meanwhile, psychologist Lewicka (2011, p. 209) 
claimed that, "Abundant research results indicate that attachment to numerous places continues to be strong", and geographer Seamon (2014, p. 44) claimed that, "Even as globalization and flows undermine some places today, these same processes strengthen other places and facilitate new kinds of places". In this manner, theories of mobility and openness may misrepresent the actual lived experiences of people, and undermine the value that they place in familiar multiscalar territorial structures (Jones, 2009).

Recognising the political and emotional importance of place, and the fact that individuals and groups still staunchly defend meaningful places along territorial boundaries, has led many geographers to seek a 'middle-ground' between theories of free-flowing networks and more rigid sociopolitical structures. Whilst Massey (2005, p. 192) defined space and place as 'open', she retained the concept of territory, and described thinking relationally as "a politics of outwardlookingness from place beyond place". Sociologists Jessop and Brenner and geographer Jones (2008) moved beyond the retention of place to suggest a polymorphic framework where territory, place, scale and network (TPSN) are all considered central to spatial relations and sociospatial organisation. Building on this TPSN framework, Jones (2009, p. 489) then advocated for the concept of 'phase space', which "acknowledges the relational making of space but insists on the confined, connected, inertial, and always context-specific nature of existence and emergence". He claimed that (p. 494), "Mobility and fluidity should not be seen as standing in opposition to territories and we should, therefore, not be forced to adopt a "networks versus territories' scenario".

Indigenous and non-Indigenous researchers in Australia have taken a similar approach to recognise diverse entities as interrelated and mobile, yet also grounded in spatial praxis and local history. Drawing on the 'gurrutu' kinship system of the Yolnu people in Australia, Bawaka Country et al., (2016, p. 460) have claimed that, "While in much literature relationality is implicated in a notion of openness, gurrutu's relationality is strongly placebased. It flows, it emerges, but not in an infinitely open way. There is structure, pattern, meaning to its emergence". In this context, emplacement is important to consider the unique histories, experiences, and patterns which structure daily life; yet, rather than framing individual experience as though it is confined to the inner-workings of a neighbourhood or city, individuals also need to be emplaced within the wider open global processes that contribute to and challenge local ways of life. 


\section{Movements, flows and moments of rest}

As indicated through the relational focus on movement and flow, humans and other spatial entities are also emplaced within time - a measurable continuum which is essential for growth, development and change (Casey, 1996; Ingold, 2008; Massey, 2005; Pink, 2015). Physicist Hawking (2009) argued that, so long as the universe continues to expand, time will always flow forward and never backward. He claimed that humans experience this arrow or direction of time through an understanding of cause and effect - the knowledge that one action sets another in motion. This continuous chronological perception of time enables individuals to draw upon memories to navigate present circumstances and events, and to consider how their experiences and actions might influence what happens next.

Relational geographies (either implicitly or explicitly) follow this arrow of time, linking the cause and effect of different place-based interactions to a process of becoming, and describing people, places, and non-human entities as always evolving and always moving toward the future (Amin, 2004; Massey, 2005; Thrift, 2008; Wright, 2015). To express the mutually-influential relationships between diverse spatiotemporal entities, Bawaka Country et al., (2016) talked about the process of becoming as 'co-becoming'. In their aforementioned research with the Yolnu people, they claimed that (p. 464), "Yolnu tell us that knowing and valuing place/space comes from living within it, learning (hearing, feeling) the language of its soils and winds and birds, and in becoming together". Geographer Thrift (2008, p. 10) also claimed that, "The human body is what it is because of its unparalleled ability to coevolve with things".

Yet, whilst movement, fluidity, and co-becoming are essential to a relational ontology, the focus on time and flow as uninterrupted, and on change and movement as constant, has also been critiqued. Geographers Marston et al., (2005, p. 423) expressed concern over "the increasingly popular practice of representing the world as strictly a jumble of unfettered flows", and Thrift (2008, p. 10) claimed that,

Embodiment includes tripping, falling over, and a whole host of other such mistakes. It includes vulnerability, passivity, suffering, even simple hunger. It includes episodes of insomnia, weariness and exhaustion, a sense of insignificance and even sheer indifference to the world. In other words, bodies can and do become overwhelmed. 
Drawing on the work of sociologist Urry (2003), geographer Jones (2009, p. 497) also claimed that cosmopolitan things, "Can knock up against each other in all kinds of interesting and networked ways, be sticky, gel, and get anchored or 'moored' through power, political strategy, and acts of social resistance. Relational entities are thus not always hypermobile". This point is salient for former refugees as, despite being relatively mobile, they may also spend a significant amount of time waiting at borders and in refugee camps, and struggle with limited mobility during resettlement (Kale \& Kindon, 2020).

When it comes to human emotional and cognitive experience, time can also become muddled. People talk about 'losing track of time', 'going back in time', and feeling like 'time is standing still'. As mentioned in Chapter Two, memories or flashbacks which are triggered by sensory stimuli (particularly those associated with PTSD, or other degenerative conditions such as dementia) can severely disrupt one's perception of time and sense of spatiotemporal continuity. Some memories may be missing or disordered, while others may be altered or intensified, leading people to feel as though they are reliving the past in the present (Hinton et al., 2004). These experiences do not indicate that time itself has stopped flowing forward, but rather that individual subjective experience does not always correlate to an objective and rational reality.

Humans also structure time by creating their own reliable daily rhythms and routines. Geographer Seamon (1980) described these rhythms as a type of social dance or 'placeballet'. Geographer Harvey (1994) also noted their choreographed nature, and claimed that rhythms and routines were socially organised and co-ordinated through the division of the day into hours, minutes, and public timetables (influencing when people work or catch public transport). Meanwhile, sociologist Lefebvre (2004) and cultural theorist Potts (2015) distinguished between social and cosmic rhythms, or as Potts (p. 551) defined it, "Between the 'linear tick tock' imposed by the punch clocks of capitalist modernity and the cyclical repetitions of nature". They claimed that everyday life is polyrhythmic, characterised by multiple overlapping beats and pulses, some of which are healthy and others pathological.

These time-structures or patterns belie the expression of place as a fleeting event or momentary occurrence (Casey, 1996; Ingold, 2008; Massey, 2005), as they bring a sense 
of stability and predictability to peoples' lives, enabling them to orientate themselves in a dynamic ever-changing world (Edensor, 2010). Individuals maintain a reasonable degree of confidence that summer will arrive at a particular time of the year, and that their favourite coffee shop will open in time for them to buy a beverage en route to the bus, which will depart at a particular hour. Whilst these rhythms and routines will subtly, or perhaps abruptly change over time, their general reliability on a day-to-day basis offers an illusion or perhaps a feeling of spatial coherence and place essence. Whilst this feeling is at odds with the rational explanation of place as emergent and always becoming something different, illusions and feelings are worth acknowledging as Thrift (2008, p. 13) claimed that, "How things seem is often more important than what they are".

Subjective experience aside, it is also evident that material entities are not always moving or changing at a constant speed, or in a uniform way. Whilst a big cosmopolitan city like London is fast-paced with extensive internal and external migrations, networks, and flows and noticeable growth and change, other places, such as the small mountainous villages in Chin State, Myanmar, are slower-paced, more homogenised, and less well connected to the international community. This does not mean that their histories are uncontested, their positionality in relation to other places is unimportant, or that landscapes are static and unchanging (far from it). Rather, it suggests that the nature of relationships between places differ, and these differences must also be accounted for. As geographer Jones (2009, p. 493) claimed, "The obvious danger of translating uniqueness into one-region-tells-all scenarios is certainly an issue for relational thinkers critically to consider”.

Factoring in the uniqueness of each relationship is also important in highlighting powerimbalances. Mutually co-becoming does not necessarily mean becoming equal. Geographer Murdoch (2006, p. 20) claimed that, "Relational space is a 'power-filled' space in which some alignments come to dominate, at least for a period of time". Thus, even relationships can be hierarchical and structured, bringing further doubt as to whether relational thinking can be post-structural, or whether elements of structure remain an important part of a relational ontology (Jessop et al., 2008; Jones, 2009; Paasi, 2004). 


\section{3:3 An emplaced model of place-attachment and wellbeing}

Drawing upon the discussion above, this final section incorporates ideas of emplacement and relationality into a visual model. The purpose of this model is two-fold, designed to guide my research practice - helping me to think through what an emplaced relational methodology might look like, and to analyse my research findings - serving as a reminder of the vast array of dynamic interrelations that influence everyday experiences of placeattachment and wellbeing. Two existing relational frameworks have strongly influenced the development of this model, including Māori health practitioner Durie's (1985) earlier mentioned wellbeing model 'Te Whare Tapa Whā', and Canadian environmental psychologists Scannell and Giffords' (2010) 'Tripartite Model of Place-Attachment'.

Te Whare Tapa Whā is both emplaced (contextualised within Aotearoa New Zealand and Māoritanga, Māori culture), and relational - linking individuals to the land, society, and spiritual realm and connecting physical, mental and emotional health (Figure 3:1). Rather than conceptualising humans as being in place, Māori ontology entails active processes of participation with place and a sense of co-constituting and co-becoming through the past, present, and future. In this manner it has similarities to other Indigenous understandings of place and place-based experience, including the Yolnu people in Australia (Bawaka Country et al., 2016) and the Anishinabek people in North America (Wilson, 2003).

Te Whare Tapa Whā is particularly significant to note in this study, as many New Zealand health policies and practitioners have drawn on Durie's (1985) model, and this holistic relational way of thinking is becoming more common in the mainstream health sector (New Zealand Government, 2018). Former refugees seeking healthcare in New Zealand will therefore likely be directly or indirectly affected by these ideas. Having grown up as a fifth-generation Pākehā with Māori relatives and Māori concepts integrated in my schooling, this model is also intertwined with my own epistemological understanding of health and wellbeing and will undoubtedly have a subjective influence upon how I analyse the research findings. 


\section{Figure 3:1: Te Whare Tapa Whā}

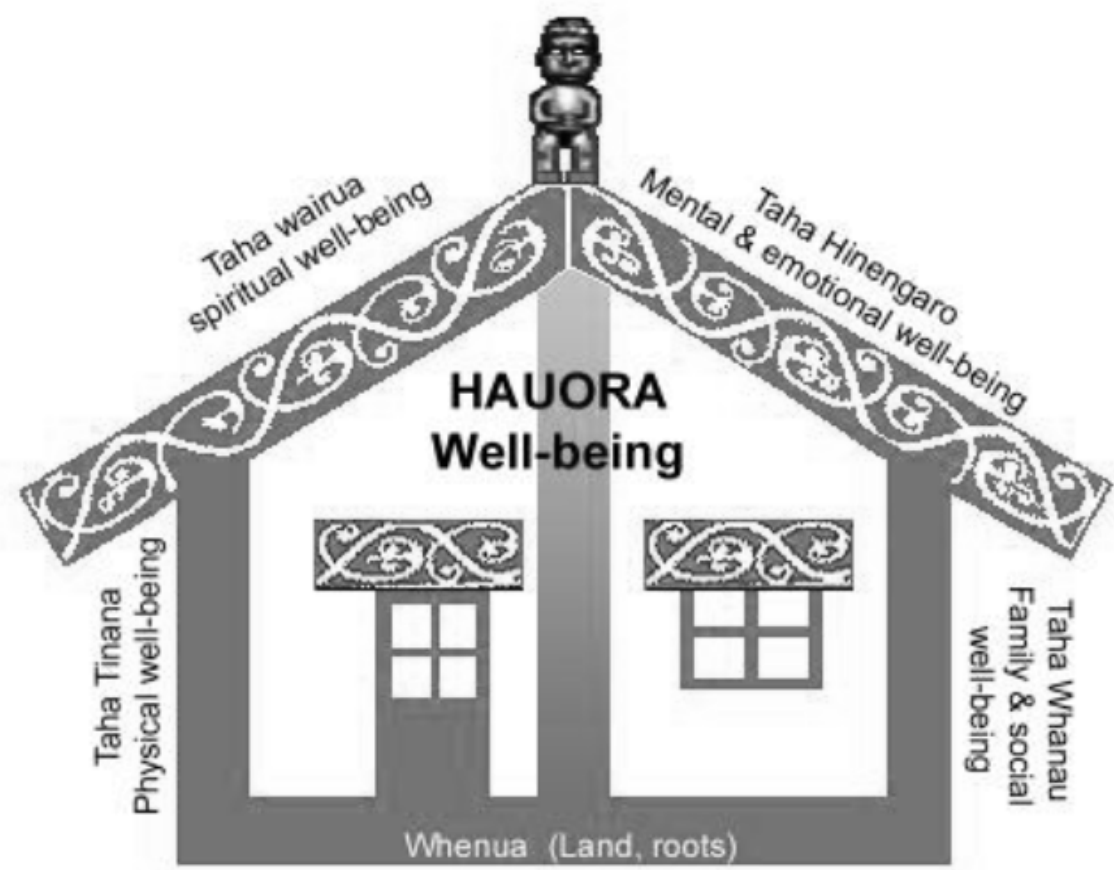

(Source: Durie, 1985)

Another influential framework has been Scannell and Gifford's (2010) 'Tripartite Model of Place-Attachment'. Integrating place-attachment theory from the late 1970 s to early 2000 s (as discussed in Chapter Two), the Tripartite Model includes dimensions of person, place, and process (Figure 3:2). The 'person' dimension focuses on who is attached, exploring individual experiences, connections, and memories, and collective experiences, meanings, traditions, cultures, and religions.

According to the authors, the 'place' dimension then concerns the 'object' of attachment and the different geographic scales at which attachment can occur (Scannell \& Gifford, 2010). Social attachment refers to an individual's connections to their family, community or wider society through social networks, shared beliefs and experiences, and feelings of familiarity and belonging. Meanwhile, physical attachment refers to an individual's connection to their surrounding environment - including both 'natural' formations and built structures (Scannell \& Gifford, 2010). Note that the terms natural and nature are contested in geography as nature may also be influenced by humans, who are themselves part of the natural world (Ginn \& Demeritt, 2008; Harvey, 1996; Smith \& O'Keefe, 1996). 
To understand the intricacies of how people connect to places, a third dimension of 'process' is included to explore the roles of affect, cognition, and behaviour.

This framework is valuable in that it acknowledges that attachments are formed between individuals and diverse environments, and through interactive feelings, thoughts, and behaviours. However, whilst the Tripartite Model provides a clear indication of key relational factors to consider in studies of place-attachment (and could be useful in studying holistic wellbeing), several shortcomings remain. The first limitation is the author's reference to place as an 'object' of affection. As mentioned above, geographers and Indigenous peoples have argued that place is not simply a passive object waiting to be explored, adapted, and loved by people. Instead it has its own agency and plays an active role in shaping human experiences and affections (Ash \& Simpson, 2016; Bawaka Country et al., 2016; Larsen \& Johnson, 2016; McCormack, 2017).

Figure 3:2: The Tripartite Model of Place-Attachment

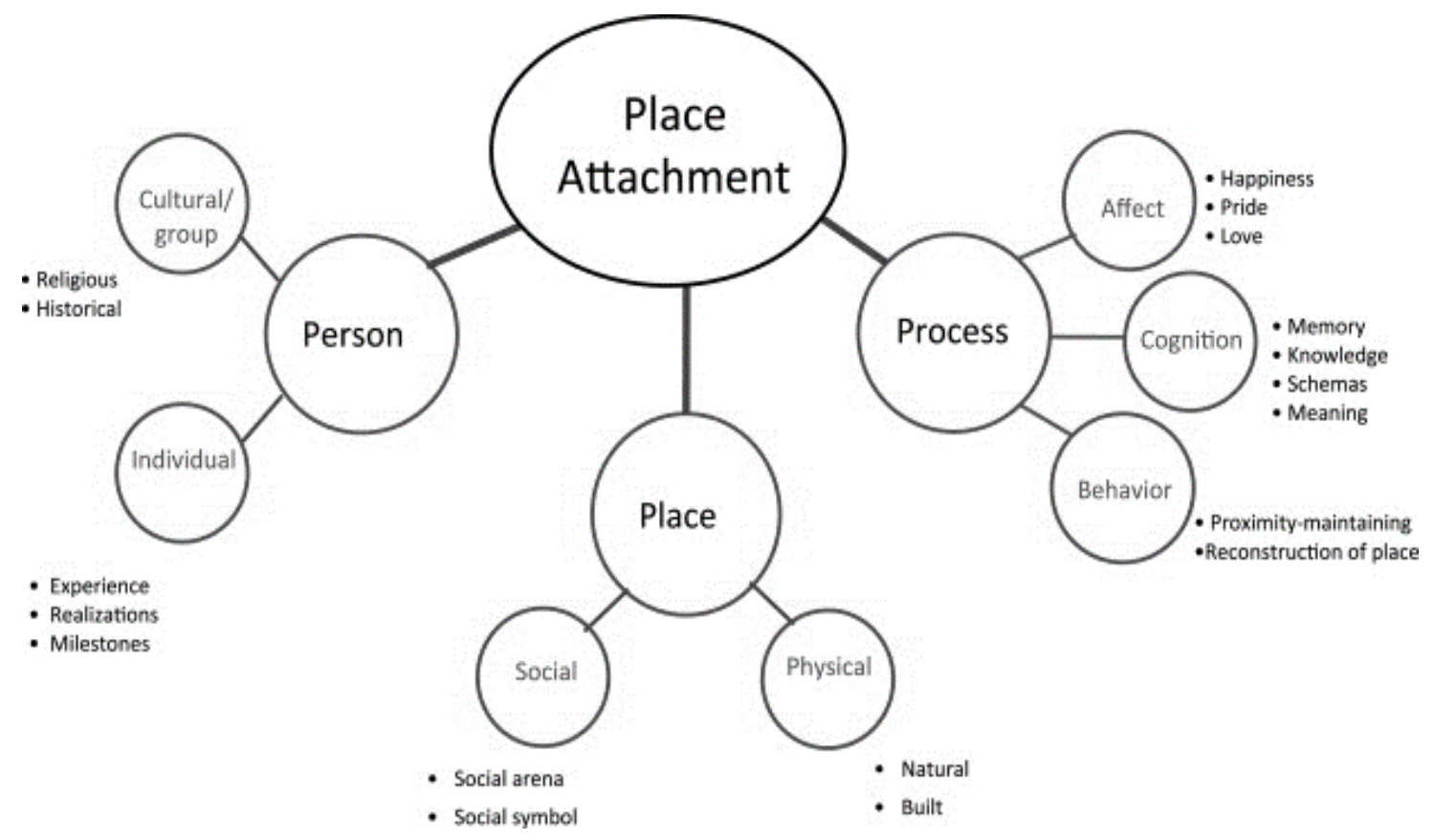

(Source: Scannell \& Gifford, 2010) 
Two key elements of the 'place' dimension are also absent, the first being the non-human element, including the flora, fauna, spiritual entities, and everyday objects that humans encounter and interact with on a regular basis (Abram, 1997; Conradson, 2005; Gorman, 2017; Swanton, 2016; Whatmore, 2002). The second missing element is 'virtual places'. As mentioned in the previous chapter, mobile communication technologies play a pivotal role in enabling individuals to maintain transnational networks and plurilocal placeattachments (Elliot \& Urry, 2010; Marlowe, 2019; McNeill, 1994). An expanding body of work in psychology and recreation studies also shows that individuals may become attached to online places (such as homes and cities created in computer games) as an escape from reality or due to games and virtual places evoking a positive emotional experience (Coulson et al., 2019; Plunkett, 2011).

The process of sensory perception is also absent, and there is a need to acknowledge that emotional experiences are not always positive (Blunt \& Dowling, 2006; Manzo, 2005). Scannell and Gifford (2010, p. 3) do discuss this in their article, yet only depict positive affective experiences within the Tripartite Model.

A final, substantial, missing element of the Tripartite Model is the dimension of time. Whilst temporal factors of place-attachment such as residence-time (Hay, 1998; Porteous, 1976; Relph, 1976; Steadman, 2006), and familiarity (Fullilove, 1996) could be explored through the dimension of process (understanding the passing of time through active participation and investment in a place), explicitly drawing out the temporalities of placeattachment is important to highlight the idea that processes are not always fluid and uninterrupted (Marston et al., 2005). People pause and rest (Thrift, 2008), memories become fragmented and disordered (Hinton et al., 2004), and, as habitual creatures, humans also create patterns and cycles, trying to maintain and recreate that which existed a fleeting moment before (Harvey, 1994; Lefebvre, 2004; Potts, 2015; Seamon, 1980). An explicit focus on time is crucial to understanding the implications of these stumbles, pauses, cycles and necessary moments of rest and reflection, and the impact that temporality has on feeling well in place.

To capture the value of Te Whare Tapa Whā and The Tripartite Model, and also include those components which are obscured or missing, I develop a new 'Emplaced Model of 
Place-Attachment and Wellbeing' (Figure 3:3). This model aims to connect the sensory, emotional, cognitive and behavioural process which shape feelings of attachment and wellbeing to the physical, social, virtual, and spiritual (or metaphysical) environment. It further aims to connect local place-based relationships to the external spatial networks and flows through which they are formed and contested, and to recognise that these spatial ensembles (Jones, 2009) co-evolve and co-become through the passage of time. Hence my inclusion of 'power negotiations' in the process dimension. Whilst useful for the purpose of analysing research data, it is important to note that this model is limited in its capacity to enhance understanding of sensory and affective experience or 'the feeling of what happens' (Damasio, 2000; Thrift, 2008). The challenge of how to communicate and understand non-representational experience in textural or visual form is explored in more depth in the following methodological chapter.

\section{Figure: 3:3: An Emplaced Model of Place-Attachment and Wellbeing}

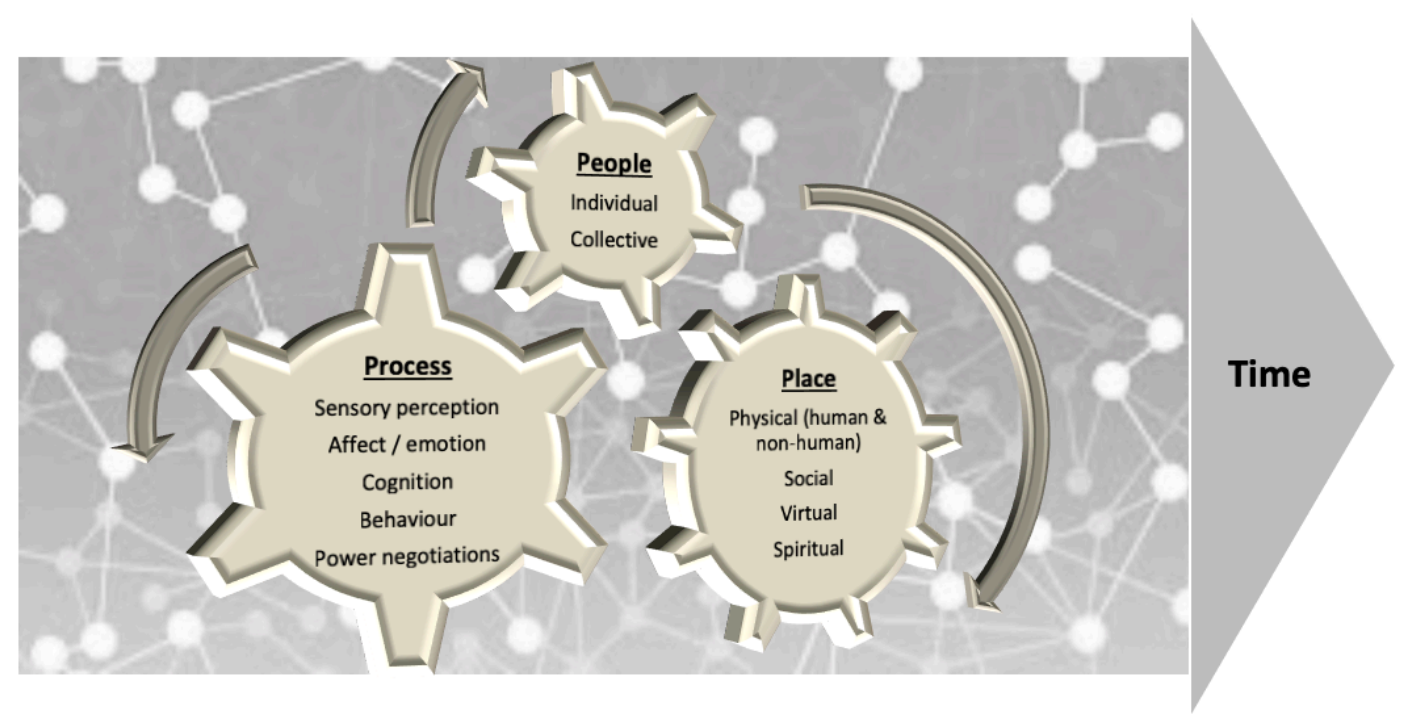

(Source: Author, 2020)

\section{3:4 Key learnings}

The key point to take away from this chapter is that individual subjective experience (in this case the experiences of former refugees) does not occur in a void. Embodied sensation, affective feelings and emotions, cognitive thought processes, and adaptive performative behaviours are relationally generated, emerging from the interactions between individuals 
and their social, spatial, and more-than-human surroundings (Bawaka Country et al., 2016; Cresswell, 2013; McCormack, 2017; Thrift, 2008). To understand how everyday multisensory experience shapes feelings of place-attachment and wellbeing for refugees resettling in Nelson, it is therefore necessary to move beyond the humanistic focus of phenomenology and embodiment to spatially and temporally contextualise lived experience - to emplace the sensing, knowing and active self (Pink, 2015; Seamon, 2018; Thrift, 2008). This emplacement can draw attention to the way that individuals affectively process and emotionally respond to different environments and form meaningful sociospatial and temporal relationships.

Because places exist in an extensive global network where people, commodities, and information regularly cross borders, encounter strange, curious, and unknown others, and (re)make taken-for-granted landscapes and local ways of life, individual experience also needs to be transnationally or plurilocally emplaced (Massey, 2005). This is particularly important in refugee resettlement research, as the literature in Chapter Two demonstrated that former refugees and migrants simultaneously negotiate multiple place-based events, relationships, and identities (Marlowe, 2019; Tolia-Kelly, 2008). Geographer Amin (2004, p. 37) also claimed that, "Increasingly, cultural attachment to a given city or region is defined through plural spaces of attachment".

Whilst some geographers have advocated for a 'flat ontology' where place scales and borders become obsolete (Marston et al., 2005), relational flows between material entities and dynamic places do not imply a process of deterritorialisation (Bawaka Country et al., 2016; Jessop et al., 2008; Jones, 2009), and at times may even reinforce existing boundaries between self and other, or perceived 'insiders' and 'outsiders'. This structural reinforcement often emerges when relationships become imbalanced, volatile, or oppressive, or when other entities are perceived to pose a threat to a perceived and valued way of life (Harvey, 1994; Murdoch, 2006). Thus, I retain the use of the terms scale, place, and territory whilst also acknowledging that places are open, interconnected, and always changing. This ontologically aligns my research with the work of Jessop et al., (2008), Jones (2009) and Bawaka Country et al., (2016). 
A final key point concerns the concept of time. I accept that, objectively, places and people are always evolving, flowing, and moving forward (Amin, 2004; Massey, 2005; Thrift, 2008). Thus, places can have no spatial coherence, essence, or permanent form. However, time, like place, is socially structured and experienced in multiple often conflicting ways. If lives play out around hourly, weekly, and seasonal routines and cycles (Harvey, 1994; Lefebvre, 2004; Potts, 2015; Seamon, 1980), and if time is felt to be moving too fast or slow or fragmented, then these subjective realities also require reflection, as they can profoundly impact how an individual feels in and relates to place. As Buttimer (1980), Lewicka (2011), Parr (2010) and Seamon (1980) suggested in Chapter Two, the loss of predictable time structures or time-space routines can be equally as disorientating and disconcerting for an individual as spatial displacement - casting time as a necessary dimension through which to frame future discussions of refugee resettlement, placeattachment and wellbeing. 


\section{Chapter 4: A Participatory Arts-Based Approach}

Building upon the epistemological understanding that human experience and knowledge are relationally constructed between individuals and their spatiotemporal surroundings, I developed a participatory arts-based research approach. This approach enabled the research participants and myself to actively engage with the places to which they felt attached in Nelson, and to co-produce creative research that was grounded in, and reflective of, everyday lived experience. Drawing upon our 'multisensory mapping project' (discussed below), this chapter highlights the benefits and challenges of doing multisensory research (Pink, 2015). Specifically, I discuss a series of preliminary 'scoping' interviews with the eleven Nelson resettlement practitioners; and meetings, site-specific interviews, mapmaking activities, painting workshops, and a public art exhibition with the twelve female Chin and Kayan participants. I also discuss processes of data coding, 'emplaced' data analysis, and research dissemination. This participatory arts-based approach was particularly valuable in moving beyond metaphorical language and the privileging of visual knowledge in Western epistemologies, and understanding how affective multisensory experience shaped participants' feelings of place-attachment and wellbeing.

\section{4:1 Research epistemology and methodology}

\section{Theorising participation in place}

To understand the multisensory qualities of place and the affective experiences, emotions, and memories which intersubjective sociospatial encounters can generate, geographers Thrift (1996) and McCormack (2005) suggested that researchers need to step back from the discursive academic world of symbolic representation and abstract theorising to centralise in-the-moment happenings, feelings, and relationships. Cultural ethnographer Vannini (2015, p. 317) also advocated for an immersive approach to research that seeks to stimulate events, perceptions, and emotions which entangle the researcher, research participants, and their wider surroundings in a mutually transformative relationship. He claimed that, "Non-representational ethnography emphasizes the fleeting, vivacious, lively, 
embodied, material, more-than-human, precognitive, non-discursive dimensions of spatially and temporally complex lifeworlds".

Such approaches are built upon the premise that, "Embodied engagement fosters knowing" (Bawaka Country et al., 2016, p. 463), and that people are always emplaced in a wider environment which influences their feelings, thoughts, and behaviours (Casey, 2009; Pink, 2015; Seamon, 2018). To conduct research that centralises emplaced relational understandings of knowledge construction, sensory researchers are adopting participatory approaches, whereby they immerse themselves within the everyday routines, rhythms, and events of place alongside participants (Longhurst et al., 2009; Pink, 2015; Spinney, 2006).

Evolving through several decades of participtory approaches in the social and environmental sciences and development studies, 'participatory geographies' gained traction in the early 2000s (Kesby, 2007; mrs kinpaisby, 2008; Pain \& Kindon, 2007; Wynne-Jones et al., 2015). Geographers Pain and Kindon (2007, p. 2809) claimed that participation is important as, "Knowledge is made through research processes rather than there being a singular version of the world awaiting detection, and participatory research not only allows for, but embraces, multiple realities". Acknowledging this plurality, Wynne-Jones et al., (2015, p. 218) added that, within participatory geographies, "A key epistemological goal has been to question and destabilise traditional barriers between 'expert researchers' and 'researched communities' to enable spaces for collaboration, negotiation and the co-construction of knowledge".

Thus, participatory research reflects a feminist commitment to sharing power with research participants and to honouring their unique knowledge, relationships, and nuanced experiences (Coghlan \& Brydon-Miller, 2014). Many participatory researchers also adopt a transformative orientation toward enhancing social justice and equality (mrs kinpaisby, 2008). However, other social scientists have critiqued the idea that participation disrupts normative power dynamics in research, claiming that the researcher is always in a position of power, and that participation may serve to enhance and legitimise harmful academic or political agendas (Cooke \& Kothari, 2001; Kapoor, 2005; Kothari, 2001). To address these valid concerns, research transparency and reflexivity is needed to highlight and negotiate 
inherent power imbalances and build mutual trust between research stakeholders (Cahill et al., 2007; Kindon, 2012).

One advantage to researchers and participants co-participating in research is that it can foster a sense of mutual recognition and 'feeling-in-common' (Wright, 2015). Thus, rather than relying upon their assumptions of what participants' described sensory experiences may have felt like, researchers can feel these experiences for themselves (Longhurst et al., 2009; Pink, 2015; Spinney, 2006). Participation also offers researchers an opportunity to enhance their spatial awareness or 'spatial sensitivity' (mrs kinpaisby, 2008), and to recognise place as an active research participant, capable of powerfully shaping research processes and outcomes (Bawaka Country et al., 2016).

Whilst spatial sensitivity arguably constitutes geography's main contribution to wider participatory research approaches (Kesby, 2007; mrs kinpaisby, 2008), senstitivity to time is also significant. Temporal factors which influence participatory research practice and outcomes may include institutional and finanicial pressure to conduct and publish research quickly, so as to be more productive. There may also be a sense of urgency around timesentitive sociopolitical issues (mrs kinpaisby, 2008), whereby researchers may feel rushed because crises are unfolding now and solutions are needed immediately. The question remains as to what impact these time pressures may have upon the building of research relationships and scientific rigour (Ferguson, 2011; Kale, 2017; O’Neill \& Hubbard, 2010). Equally pertinant is the question of how time is felt by researchers, participants, and nonhuman entities - a point which I return to later in this chapter.

\section{Positioning the researcher as apprentice}

In practice, multisensory participation enables the researcher to become 'similarly situated' to the research participants; to effectively see through the eyes of another or walk a mile in someone else's shoes. Anthropologist Pink (2015, p. 59) claimed that,

By attempting to become similarly situated to one's research participants and by attending to the bodily sensations and culturally specific sensory categories (e.g. in some, but not all cultures, smell, touch, sound, vision, taste) through which these feelings are communicated about and given value, ethnographers can come to know about other people's lives in ways that are particularly intense. 
In this manner, the role of a participatory researcher has been likened to that of an apprentice, who observes a particular skill or behaviour and learns the significance of different actions through practice (Ingold, 2000; Pink, 2015).

Understanding the researcher as an apprentice provides a novel way to reconceptualise the power dynamics between researchers and participants. In traditional research relying solely on interviews and observation, the subjectivities of the researcher are prioritised throughout the research process as they design, facilitate, and analyse the questions, methods, and data. Yet, through apprenticeship, the participant leads the researcher to reposition their vantage-point and enable them to become aware of new ways of feeling and interpreting their surroundings. Thus, knowledge is intersubjectively constructed as the researcher realigns their way of perceiving and thinking to more accurately reflect the experiences of those being studied. This dynamic process requires an open (in Massey's 2005 sense of the word), unbounded methodological framework, whereby the specific methods used rely in part upon the direction of individual participants, as well as the opportunities or limitations imposed by the surrounding environment. Sensory participation researchers may thus have methods in mind, but also be open to new ways of doing research, and to moments of surprise and unpredictability (Hahn, 2006).

For multisensory researchers, conventional techniques such as interviewing can be limited. Geographer Conradson (2005, p. 346) claimed that, "In addition to inevitable difficulties with recall of past events, there is a sense in which the embodied and emotional immediacy of particular relational encounters may be elided or even lost in a subsequent retelling. The account is always given after the event". One of the ways in which he suggests that this issue might be addresed is to, "Accompany an individual during their engagement with a landscape, perhaps joining them on a walk for instance, whilst being attentive to the immediate and more gradually emergent effects of these encounters".

Walking has become one of the main methods in participatory sensory research. In France, the 'Centre de recherche sur l'espace sonore et l'environnement urbain' (CRESSON), which is based in the School of Architecture in Grenoble, has produced a large body of work using methods of 'urban transects' (Melemis \& Tixier, 2010). Whilst the concept of urban transects is relatively flexible, for CRESSON this method generally involves 
researchers walking alongside locals through different landscapes to capture different 'ambiences' - physical characteristics and nuances of place, and 'atmospheres' - feelings derived from unique times and spaces (Adey et al., 2013). These ideas may be traced back to the romantic notion of the 'urban flaneur', perusing through the city simply for the pleasure of coming to know its sights, sounds, smells, tastes, and textures (Murail, 2017).

Transect walks are also popular in the UK. In 2008 geographer Tolia-Kelly facilitated a study with migrants walking together and exploring their sensory affective experiences in the Lake District, and in 2010 sociologist O'Neill and geographer Hubbard implemented an 'ethno-mimesis' project whereby refugees and new arrivals guided residents in a walking event through the city in the English East Midlands. They claimed that (p. 46),

While walking should not be privileged as a way of knowing, it has certain sensate, embodied, relational and collective attributes which rendered it particularly useful as a means of exploring the importance of being-in-place among a group whose lives are often depicted as markedly transnational.

Thus, walking with participants enabled the researchers to become aware of the specific landmarks, objects, and events that captured the attention of individuals, and to better understand the effects different stimuli had on personal relationships with unique places.

Other mobile approaches have included cycling and dancing. By training and riding with racing and touring cyclists in the UK and France, geographer Spinney (2006) was able to see the sights of the cycleway himself and feel the rhythms, pain, and elation that comes from intense physical activity. He was thus more attuned to the vestibular, proprioceptive, and interoceptive experiences described by the other cyclists, sensing the movements of the bicycle, feeling the rhythm of the journey through his muscles and joints, and understanding the physiological condition of the body-in-motion. Meanwhile ethnomusicologist Hahn (2006) explored how culture was embodied through Japanese dance, and geographers Thrift (2000) and McCormack (2008) have explored dance as an affective embodied way of knowing oneself and others.

Researchers have also explored sensory participation in-situ. On their website, the 'Slow Research Lab' (2018), a multidisciplinary research and curatorial platform based in the Netherlands, claimed that, 
Slow Research Lab brings its knowledge and network into deep, site-specific engagement with locations that have special resonance with our mission and areas of inquiry. In-situ dialogues are short-term, intensive 'activations' of those sites through curated discussions, performance, sensory activations, food experiences, and more.

Rather than moving through and comparing different spaces and places on any given route, these researchers focus on a single destination selected for its pre-existing value. Another participatory study that focuses on activating specific sites is geographers Longhurst, Johnston, and Ho's (2009) research, whereby migrant women in Hamilton, New Zealand cooked traditional food from their native countries in their homes, and ate together with the researchers. Choosing whether to adopt mobile or site-specific participatory methods may come down to the type of activities that one wishes to focus on, as cycling inevitably entails movement and eating is generally enacted in-situ.

Yet, becoming similarly situated to participants does not necessarily qualify the researcher to know what others are feeling or thinking. Some form of interpersonal communication is still needed to share individual subjectivities and understand the similar and dissimilar ways that people interpret shared experience. Anthropologist MacDougall (2005, p. 116) claimed that in order to comprehend what others are trying to convey, "We may need a 'language' closer to the multidimensionality of the subject itself - that is, a language operating in visual, aural, verbal, temporal, and even (through synesthetic association) tactile domains". 'Language' may be a confusing term to use here, seeing as MacDougall is making a clear distinction between linguistics and other material forms of communication. However, the idea that affective multisensory experience can be expressed non-verbally opens up new possibilities for understanding lived experience in research.

\section{Communicating multisensory experience through art}

In the social sciences, art practice, particularly that which employs forms of visual creation such as photography, video, sketching, painting, and map-making, has become a popular way to communicate lived experience (Bartos, 2013; Jones \& Jam, 2016; Kale, 2018; Kindon, 2016; O’Neill \& Hubbard, 2010; Sensory Journeys, 2017; Tolia-Kelly, 2008). In geographer Tolia-Kelly's (2008) nurturing ecologies project with migrants in the UK, and geographers Askins' and Pain's (2011) UK research with young people of African and 
British heritage, participants and artists produced paintings to share their sociospatial experiences with the researchers. My Masters' research with former refugees and host society residents in Wellington also used painting to explore participants' multisensory experiences and negotiations of home and belonging (Kale, 2017; Kale et al., 2019).

Another project exploring multisensory experience was facilitated by artist Nold and used practices of map-making. Nold collaborated with the UK sustainable transport charity 'Sustrans' and four schools in Bristol in 2016 to facilitate a year-long project which focused on the question, 'Do people have different sensory experiences and emotional relationships with their local area depending on their mode of travel?' (Sensory Journeys, 2017). Participants drew their sensory experiences on a map of their school transport route (Figure 4:1), providing a novel way to relate to specific places and activities.

Figure 4:1: Sensory Journeys

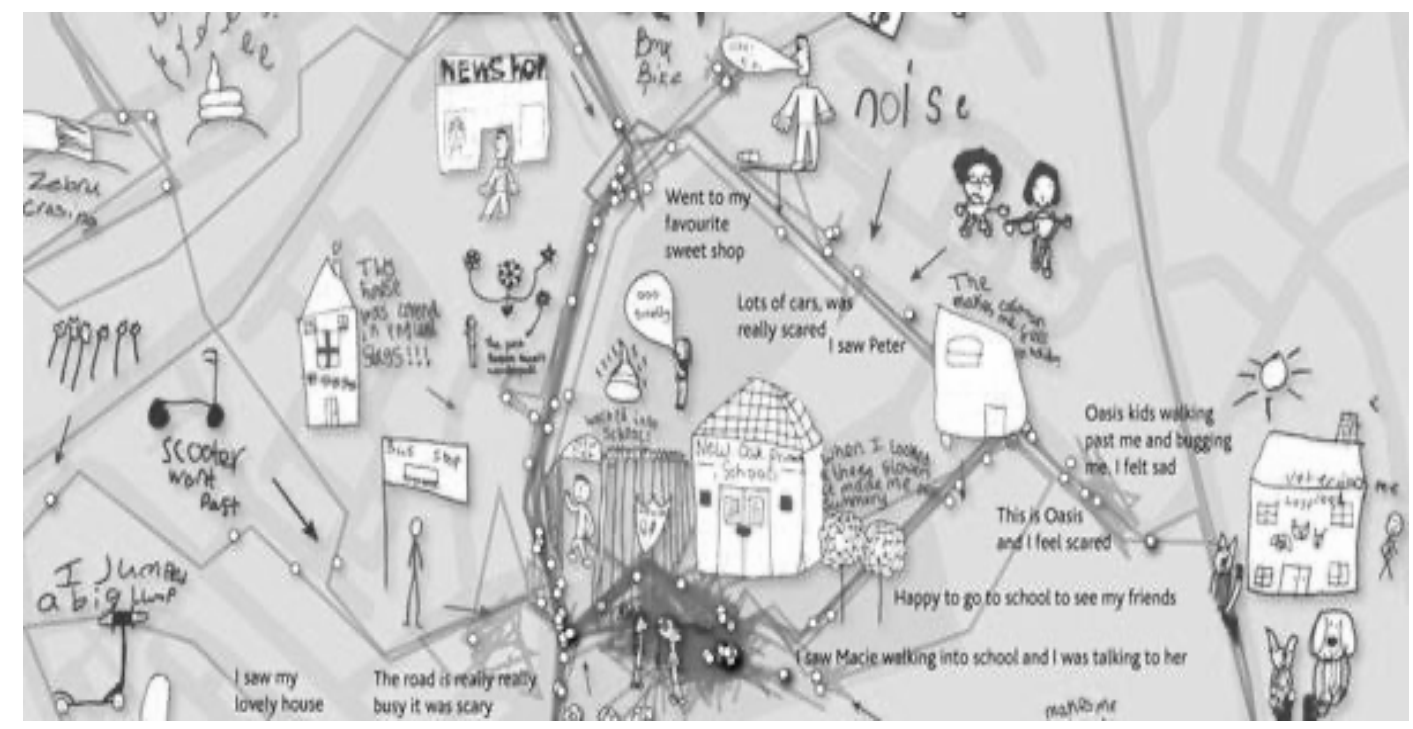

(Source: http://www.sensoryjourneys.net/)

Meanwhile, other mapping techniques have centralised the subjective self and focused on affective emotional experience. This was evident in migration researchers Oliveira, Meyers, and Vearey's (2016) 'Queer Crossings' project, where participants mapped their place-based experiences onto bodies (see Figure 4:2). Both Nold and Oliveira et al.,'s mapping approaches are valuable in relational research where direct embodied experience and more-than-human places are being explored together. 
These visual methods are particularly useful in working with people who speak different languages, as they provide an alternative (non-verbal) means of communication (Ferguson, 2011; Ramos, 2004). Creating artwork can also enable individuals to choose how they portray themselves to others. For former refugees, who are unfairly homogenised and misrepresented in dominant political and media discourse, this agency or 'artistic license' is important in deconstructing harmful refugee stereotypes and presenting nuanced counter-narratives which demonstrate individuality and feeling. Such counter-narratives have been produced by former refugees in Wellington (Kale, 2018); elderly street artists in Lisbon (Lorenz, 2015); and prisoners in Lithuania (Hicks et al., 2017). However, emotive artwork may also generate feelings of pity (Pussetti, 2013), and reinforce negative stereotypes of refugees as powerless and traumatised (Ford, 2012).

Sociologists Lyon and Back (2012) have also argued that, when an individual is collecting and generating sensory data, they are focused on specific stimuli and other senses may inadvertently be obscured. Yet, geographer Bartos (2013) has challenged this suggestion in her photography research with New Zealand schoolchildren, claiming that the participants in her study were aware of multiple senses even when they were focused on their cameras, and that the photographs taken and shared were imbued with other sensory memories, providing significant insight into participants' multisensory relations with the places they were portraying. A similar phenomenon was observed in my Masters' research, where the visual and tactile method of painting did not obscure other senses, but rather elicited multiple sensory perceptions and memories (Kale, 2017; 2018). These studies indicate that multisensory experience is not necessarily limited during art-based research; however, it remains important to consider how the tools being used may influence unique experiences, memories, and representations (Askins \& Pain, 2011).

There are also limitations to using visual methods when trying to convey a multisensory experience or a specific taste, sound, smell, texture or feeling. As mentioned in Chapter Two, there have been multiple critiques in geography of visual experience being prioritised at the expense of the other senses (Brown, 2016; Gorman, 2017; Porteous, 1990; Relph, 1994; Rose, 1993). In a quest to move beyond the visual, smellscape researcher McLean (2017) adopted a mixed-media approach whereby participants created tactile smell-maps of different cities (Figure 4:3). 
Figure 4:2: Body Mapping

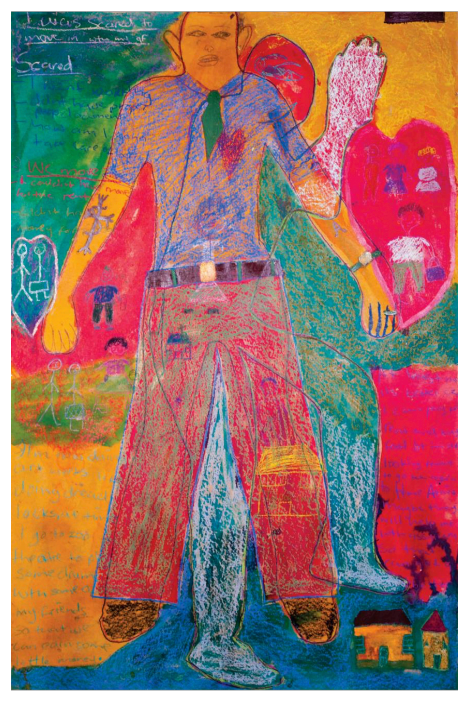

(Source: Oliveira et al., 2016)
Figure 4:3: McLean's Marseille Smell-Map

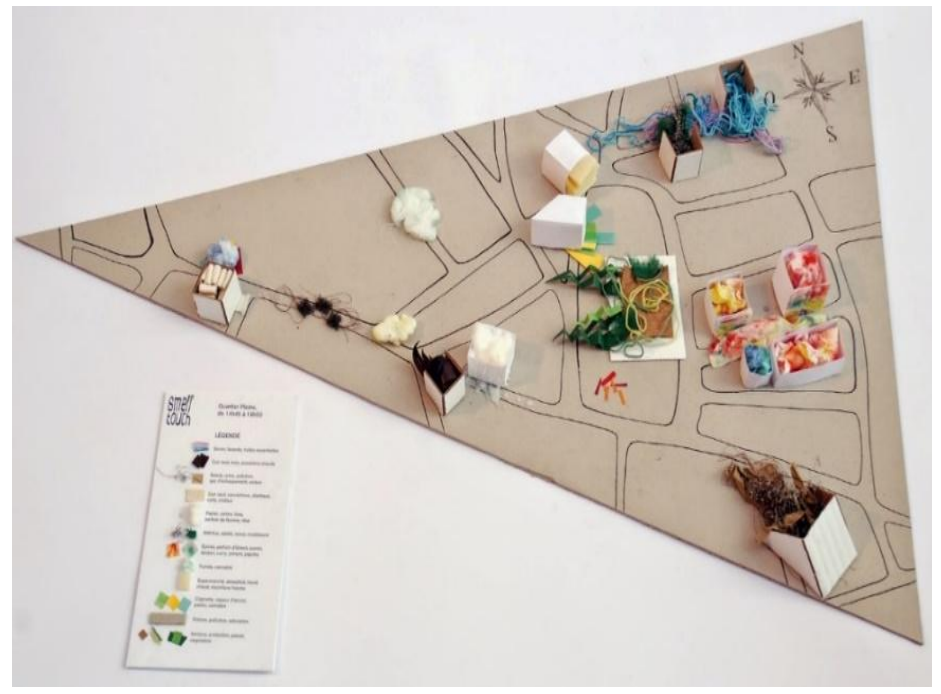

(Source: McLean, 2017)

Other researchers have opted for multi-media approaches, using audio and video as ways of navigating and exploring place (Butler, 2007; Gallagher \& Prior, 2014; Lobo \& Barry, 2019; Pink, 2015). Anthropologists and media theorists have argued that moving audiovisual images can evoke multisensory memories for viewers, who relate their own experiences to those onscreen (Marks, 2000; Pink, 2015). In this manner, viewing a video can stimulate both psychological and corporal experience (MacDougall, 2005), and increase awareness, compassion, and a sense of intimacy towards others (Biella, 2008). In geographers Lobo and Barry's (2019) research with former asylum seekers and refugees in Australia, participatory video was also used to explore participants' subjective embodied experiences and transgress barriers between participants, researchers, and the wider community. However, these approaches also fall short of capturing specific nuanced multisensory stimuli, as the viewers' memories and imaginings will not necessarily open them up to new cultural or geographical smells, flavours, textures, and temperatures.

Drawing upon participatory researchers' recognition that a wealth of knowledge lies outside of academia (Wynne-Jones et al., 2015), I also explored multisensory community exhibitions for ideas on how to move beyond the visual. During a 2018 trip to San Francisco, California, I visited a museum called the 'Exploratorium'. Here the public interact with exhibits for an embodied experience which connects them to the processes of 
creating art, technologies, and cultures, rather than offering disembodied abstract representations of the world (Exploratorium, 2018). Building on this hands-on approach, I decided to facilitate a $\mathrm{PhD}$ project where the participatory semiotic academic methods of creative mapping and painting could be coupled with the generation and collation of other sensory objects and displayed in an interactive multisensory exhibition.

\section{4:2 The multisensory mapping project}

\section{Aims and objectives}

Between May 2018 and March 2019, I facilitated a PhD project in Nelson which I refer to as, the 'multisensory mapping project'. Framing this project as a distinct phase of the $\mathrm{PhD}$ research made it clear to prospective participants what their involvement would entail. Adopting a relational epistemology and participatory arts-based methodology, as discussed above, I aimed to work with local refugee resettlement practitioners and former refugee participants to co-produce creative research that was sensitive to place, time, culture, and power dynamics. To address the research questions outlined in Chapter One, the project had three key objectives. These objectives were for me as the researcher to:

1. Draw upon the experiences, knowledge and expertise of Nelson resettlement practitioners to learn about the current approaches and practices being employed to support former refugees as they resettle

2. Work with participants to learn about their everyday multisensory experiences of place-attachment and wellbeing in Nelson

3. Experiment with immersive, creative new methods to communicate and disseminate embodied multisensory experience

These project objectives also related to my wider $\mathrm{PhD}$ research objectives, which included contributing to theoretical discussions on refugee resettlement, place-attachment, and wellbeing; expanding methodological ideas around designing, facilitating, and analysing emplaced multisensory research; and practically addressing local concerns around refugee mental health issues and a lack of mental health care in Nelson (Nelson Multicultural Council, 2012; Ward et al., 2018). 


\section{Preliminary 'scoping' interviews}

After obtaining University ethical approval at the end of May 2018 (Appendix One), I travelled to Nelson to begin generating research data. My first step was to conduct a series of preliminary 'scoping' interviews with resettlement practitioners to explore how placeattachment and wellbeing were (or were not) being attended to in Nelson refugee resettlement. I also questioned how local initiatives were familiarising former refugees with their new multisensory environments. Practitioners' local knowledge and expertise helped me to guide my research design and ensure that the research would be beneficial for those involved (Pain \& Kindon, 2007; Participatory Geographies Working Group, 2020; Wynne-Jones et al., 2015).

In total, one month was spent contacting practitioners and conducting and transcribing their interviews. After researching resettlement organisations online, I visited the Red Cross, Nelson Multicultural Council, Victory Community Centre, and English Language Partners, to introduce my project and invite managers and staff members to participate in hour-long semi-structured interviews (Dunn, 2016; see Appendix Two). Key informants from these organisations then helped me to connect with an individual who worked with refugee-background families at a local school, a self-employed psychologist, two occupational therapists specialising in holistic approaches to wellbeing, and a group of local residents who run a workspace called Community Art Works. An informal meeting was held at Community Art Works to discuss potential painting spaces for the project, and ten resettlement and wellbeing practitioners volunteered to be formally interviewed. Between them, these practitioners had a broad range of experience in housing former refugees, enhancing access to education, employment, transport, and healthcare, and supporting social interactions and involvement in culture and the arts.

Due to the professional nature of the information being shared, interviews were facilitated in participants' offices. The manager or an acting representative from each organisation was asked to sign a consent form giving permission for their staff to be interviewed and for the names of their organisations to be published, and each individual signed a consent and confidentiality form before I began an audio-recorded interview (Appendices Three and 
Four). To protect confidentiality, resettlement practitioners are not named in this thesis and are referred to by the organisation they work for or the services they provide.

Through the relationships and rapport formed during these interviews, practitioners became invested in the project and played a key role in connecting me to local refugeebackground communities and helping me to recruit participants (Bradshaw \& Stratford, 2010). Their early involvement paved the way for continued interest and later reengagement with research outcomes, and will be important in enabling the research to be practically applied (mrs kinpaisby, 2008).

\section{Participant recruitment}

The second stage of data generation began in November 2018 with the recruitment of participants. To generate interest in the project I produced a flyer with key details and requirements (Appendix Five). The project was advertised under the title, 'A Sense of Nelson' and the flyer stated that participants must be a former refugee from Myanmar; have New Zealand permanent residency or citizenship; live in Nelson; identify as female; be aged thirteen or over; and have basic English. Two family members then volunteered as interpreters, enabling women with limited English to also participate. Some comments may be indirect as a result of this interpreting, and particular responses may have been withheld by the women to avoid embarrassment or family tensions (Temple \& Edwards, 2006).

Considering legal status was important as individuals who do not have New Zealand permanent residency or citizenship (including short-term residents, tourists, and asylumseekers), may face unique challenges in developing attachments to places they may soon be leaving. Whilst attachments are not always dependent upon residence time (Kale, et al., 2019; Lewicka, 2011), including these experiences alongside the experiences of people who are relatively settled in Nelson would make for a more complex study. Therefore, I focused solely on those who had the legal right to stay in New Zealand. This temporal distinction also led to the criterion that participants must live in Nelson City, as visitors passing through or stopping to work awhile might experience Nelson in different ways than local residents (Hay, 1998; Porteous, 1976; Relph, 1976; Steadman, 2006).

The decision to work with girls and women was partly grounded in resettlement literature, which demonstrated that refugee-background women are more likely than men to be 
isolated in their homes because of limited access to transport, domestic responsibilities and limited English language ability (Bose, 2014; DeSouza, 2011; Kale \& Kindon, 2020; Martin, 2008; Swe, 2013; Urry, 2012). By bringing female former refugees out of their homes into a participatory research process within Nelson City, I hoped that their confidence might be enhanced and their relationships with the city might be strengthened. Resettlement practitioners also indicated that women may feel more comfortable discussing ideas of embodied sensory experience with a female researcher than men, although as sociologist Oakley (2016) mentioned when reflecting upon critiques of her own feminist research practice, being female doesn't necessarily mean being relatable to participants of the same gender, as intersections of race and class may also play a role in casting the researcher as an 'insider' or 'outsider'.

To distribute the recruitment flyer, I asked resettlement practitioners to share copies through their networks. I also posted an image of the flyer on Facebook and asked for Nelsonians who had contact with individuals from Myanmar to send me a message. This outreach resulted in two months of meetings, coffees, e-mails, and phone calls with 'people-who-knew-people', until I eventually connected with three key informants who passed on the details of potential participants. This networking approach was effective as former refugee communities were familiar with the organisations and individuals approaching them and knew them to be trustworthy. Thus, my research came across as credible and my key informants could vouch for the research integrity (Bradshaw \& Stratford, 2010). Whilst I initially had concerns over the length of time it took to connect with willing participants, time was crucial in fostering strong relationships with the Nelson community and garnering support for the research (mrs kinpaisby, 2008). After receiving details of individuals who were interested in participating in the project, I contacted each girl and woman via phone with a research invitation. By late January 2019, twelve people had volunteered to participate, including Mu Pli, Ruth, Little Ruth, Ngai Ciang Sung, Mai Aye Aye, Sui Sung, Elizabeth, Ngun, Zai Zai, Jenavi, and Sui Tin Men.

\section{Initial meetings and focus group}

I began the project by facilitating two separate hour-long meetings with Mu Pli, Ruth, and Little Ruth at their homes. These participants became involved earlier than the rest of the 
group as Mu Pli and Little Ruth were both students and needed to finish the project before starting polytechnic and college (high school) in early February (see Appendix Six for meeting, interview and workshop schedules). I then facilitated a focus group with the nine other participants at Ngai Ciang Sung's home in Toi Toi near the Nelson CBD.

These meetings each followed a similar format. After making our introductions, I shared information about the research and time was set aside for any questions or concerns that participants had regarding the research methods or practices that may be culturally sensitive (Dowling, 2016; Howitt \& Stevens, 2016; Kindon, 2016). Once the girls and women were satisfied with their expectations of the research, I helped them to sign consent and confidentiality forms, clarifying any text that they were unsure of (Appendix Seven). Participants were under no obligation to sign the consent forms or continue with the research if they wished to cease involvement at this stage. It was made clear that participation was voluntary and individuals were free to leave or retract their data an any time up until the $30^{\text {th }}$ April 2019, after which time I planned to begin writing up the research findings and preparing conference presentations and journal articles.

Demographic questions were also filled out to provide insight on who the participants were, where they came from, and what types of activities they regularly engaged in around Nelson City (Appendix Eight). This information was useful in helping me to understand the commitments that participants were juggling alongside the research, and to consider how we might weave the interviews and workshops into their busy education, work, childcare, and social schedules. Demographic information also provided key contextual detail to be analysed alongside the data generated through other research activities.

During the meetings and focus group (and many of the later interviews and workshops) we shared food which I provided, or which participants offered to me as a guest in their homes. Eating together created a relaxed atmosphere and enabled participants and myself to become similarly situated through shared visceral experience (Hayes-Conroy \& HayesConroy, 2010; Longhurst et al., 2009; Pink, 2015). This shared experience helped us to build rapport, and, as was evident in my empirical Honours and Masters' research, data collected and generated under these circumstances was far richer than in activities where food and drink was not provided and the process was treated more formally (Kale, 2017). 
I then worked with participants to help them to map their experiences of displacement and place-attachment onto street maps of Nelson (Figure 4:4), assisting with English vocabulary and spelling when needed. The boundaries of the map used for this activity roughly aligned with the official Nelson City boundaries (though were slightly off due to the difficulty in accessing appropriate maps for the purposes of the project). This parameter, "Covers a land area of $442 \mathrm{~km}^{2}$ from Champion Road, Stoke in the southwest, where it has a boundary with Richmond in Tasman District, to the Bryant Range in the east and Cape Soucis (Raetihi) in the north" (Nelson City Council, 2018). Drawing upon their memories of past experiences in Nelson, participants made notes to explain the multisensory stimuli that they had encountered in these places and how these stimuli had made them feel. Whilst there may have been limitations drawing on memory (and potentially forgetting, romanticising, or denigrating particular places), these were familiar places that participants regularly visited and were layered with meaning (Fullilove, 1997; Hage, 1997; Mathisen \& Cele, 2020), making them memorable.

Figure 4:4: Street Map of Nelson City

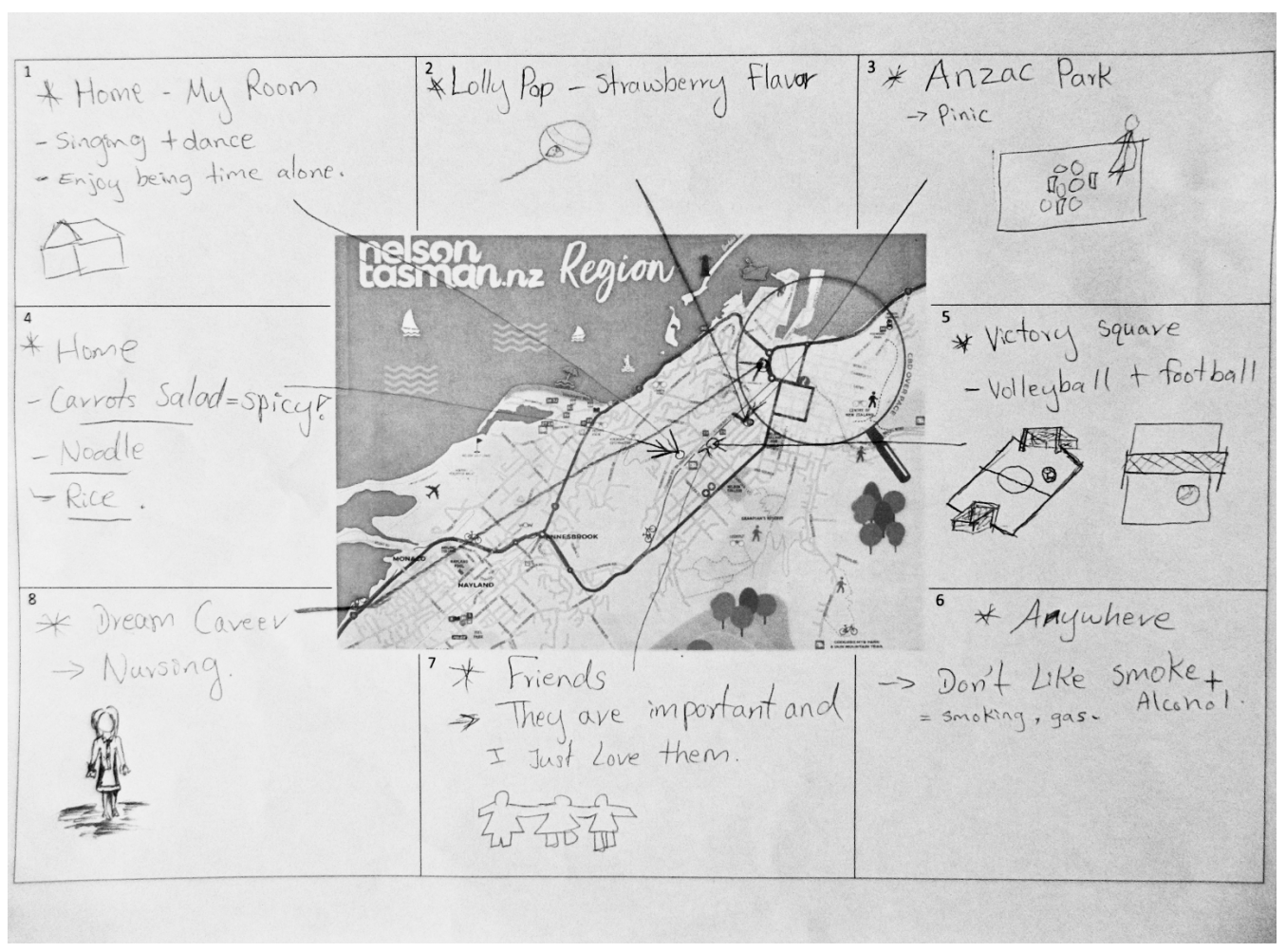

(Source: Map by Mu Pli) 
I then encouraged participants to choose one of the places to which they felt attached to visit with me for an individual site-specific interview. They selected a date and time that suited them to meet, and I asked them to consider the types of activities that they normally engaged in within that place so that we might pursue these activities together. These choices enabled participants to have greater control over the research process, and challenge some of the power imbalances inherent in participatory research (Kesby, 2007; Thomas et al., 2015; Valentine, 2005). I then coordinated with participants to make the necessary arrangements for transport and organised any resources needed for the activities before meeting each individual again in person.

Participants also mapped and discussed places that they visited outside the city in the wider Nelson / Tasman region. Whilst we didn't have the time, funding, or transport available to make overnight excursions to visit these places together, I later collated their city maps and made a large regional map on which all of the visual data of place-attachment was represented (Appendix Nine). This process enabled me to see that the places to which participants felt attached were not only concentrated around homes and neighbourhoods (the most commonly researched scales of place-attachment; Lewicka, 2011), but spread across the region, indicating a significant level of mobility and variability within the types of environments which had become meaningful.

\section{Site-specific individual interviews and multisensory mapping activities}

Whilst participants choosing different places for their interviews made for a 'messy' approach to fieldwork in terms of logistics, planning, and methodological consistency, this

approach was integral to understanding their everyday lives and unique place-based experiences. During the interviews, questions were asked regarding the specific places in Nelson to which participants felt attached, or displaced. All interviews were audiorecorded, except for one where the participant chose to have her answers recorded by hand (Dunn, 2016; Valentine, 2005). Drawing on ideas from Oliveria et al.,'s (2016) mapping project, I also worked with participants to help them to sketch or write down the multisensory stimuli they perceived, and the memories and feelings these stimuli evoked, onto a non-descript outline of a body (see Figure 4:5). Photographs were taken with participants' permission. 
Figure 4:5: Site-Specific Body Map

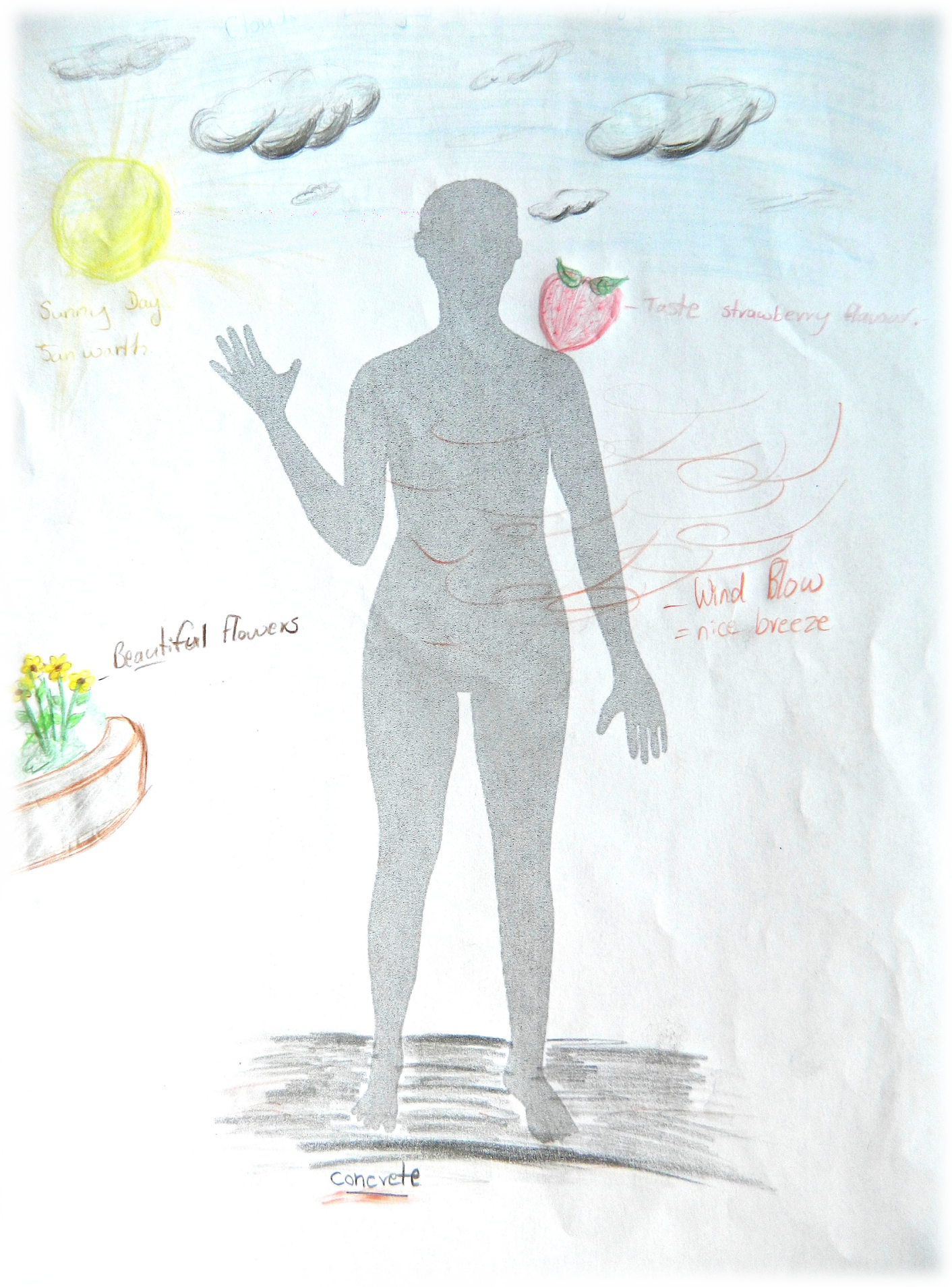

(Source: Body map by Mu Pli) 
My first interview was with 19 year old $\mathrm{Mu}$ Pli, whose family had relocated to New Zealand through the family reunification programme six years earlier and acquired citizenship. Mu Pli had recently finished College and applied to start a course in Nursing at the local Nelson Marlborough Institute of Technology (NMIT). She also was involved with a worship team at her church and acted as a community youth leader. We met at her house in Toi Toi and did the interview in her living room over a shared lunch before waking to one of her favourite places, ANZAC Park, in the CBD (Figure 4:6).

Several days later I met with 28 year old Ruth and her 14 year old niece Little Ruth at their new home in Stoke (Figure 4:7). Ruth and her husband had come to New Zealand as quota refugees five years prior to her interview and were New Zealand permanent residents. Ruth was a stay-at-home mother to three young boys and a caregiver to Little Ruth, who had arrived in New Zealand two years later through the family reunification process and was also a permanent resident. Unfortunately Little Ruth's parents and other relatives remained in Myanmar and there was some concern over their welfare. Aunt and niece chose to do their interview together at their house, where Ruth's husband was available to translate when needed and she could breastfeed her baby and keep an eye on her young sons.

My next interview was with 33 year old Ngai Ciang Sung and her neighbour, 42 year old Mai Aye Aye. Both women were originally from Chin State, had come to New Zealand as quota refugees over twelve years ago, had obtained citizenship, and lived in Toi Toi with their husbands and children. A place that Ngai Ciang Sung felt attached to was her family home (Figure 4:8). During the interview, the three of us sat on the living room floor and shared coffee and home-made 'Chin-style' doughnuts. Ngai Ciang Sung's young children played as we talked. She explained that her home was important because it made her feel safe and relaxed; however, she also enjoyed being outside gardening and camping.

After the interview, Mai Aye Aye took me next door to visit her kitchen (Figure 4:9) where she enjoyed cooking and looking out the window at the beautiful views of the hills. She said that she hoped to one day own her own food cart selling traditional Chin food; however, in the meantime she enjoyed going into the city to eat Thai food. 
Figure 4:6: ANZAC Park

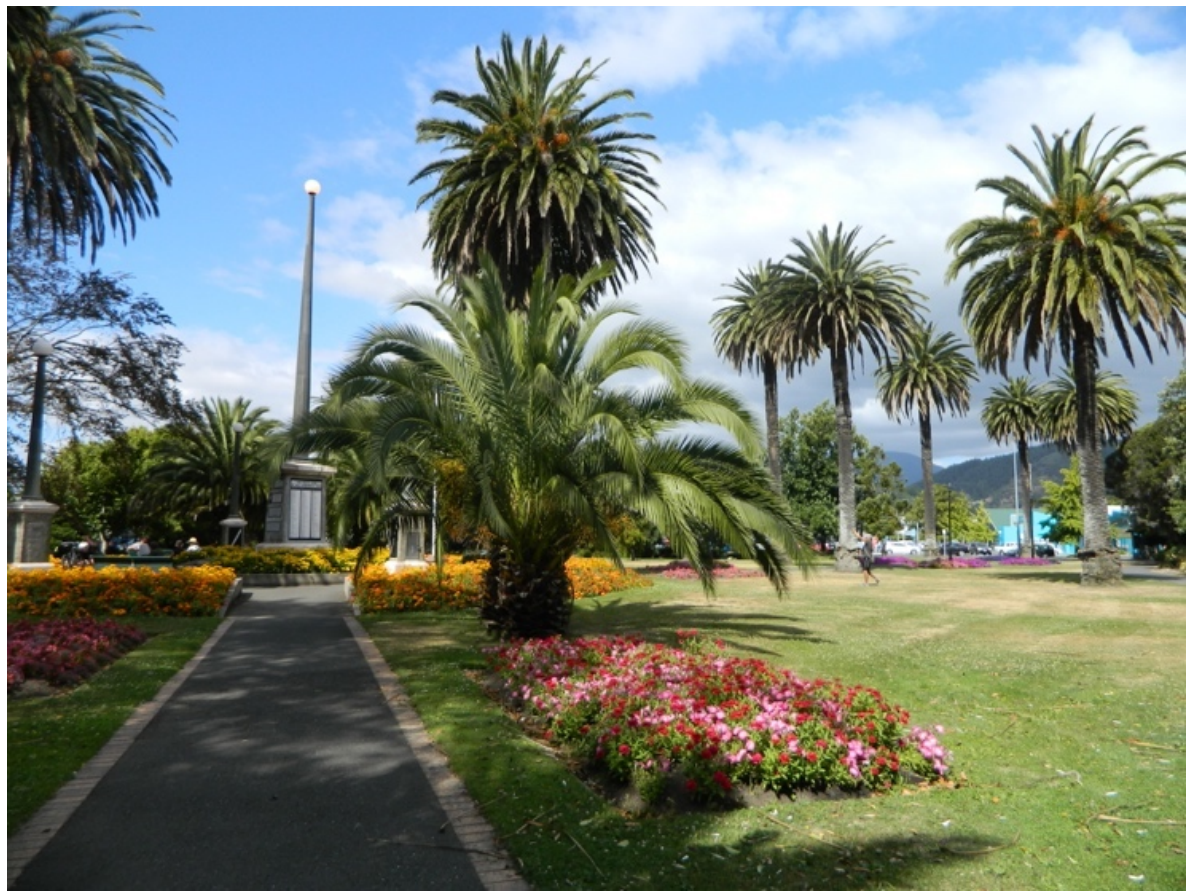

(Source: Photo supplied by the author, 2019)

Figure 4:7: Ruth and Little Ruth's House

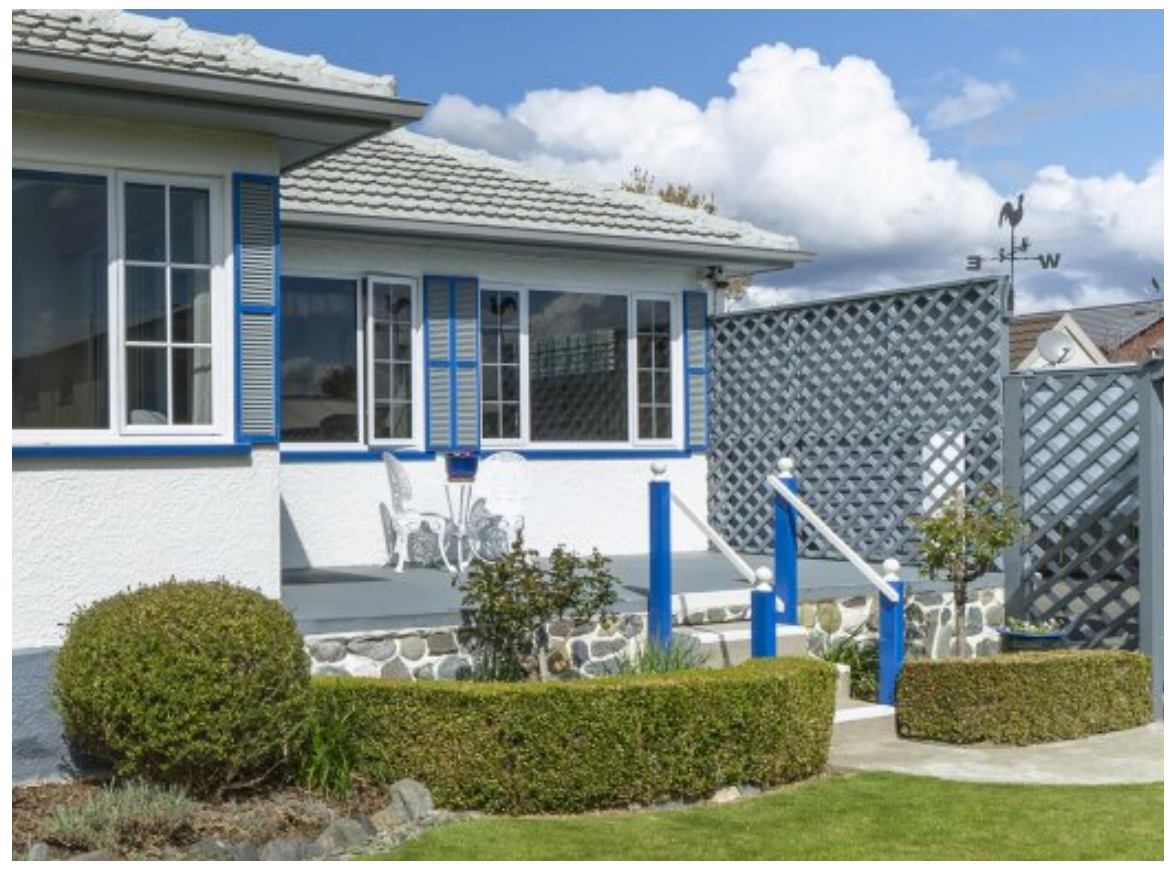

(Source: Photo supplied by the author, 2019) 
Figure 4:8: Ngai Ciang Sung's Living Room

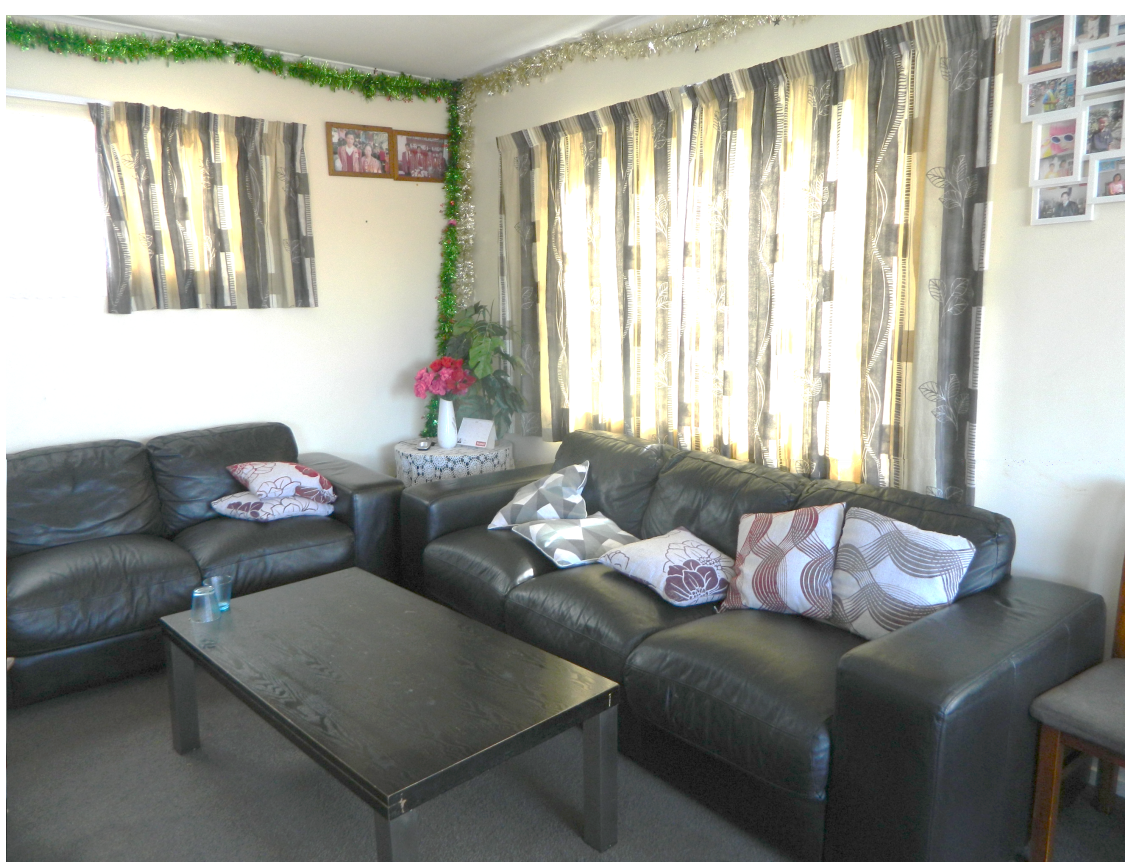

(Source: Photo supplied by the author, 2019)

Figure 4:9: Mai Aye Aye's Kitchen

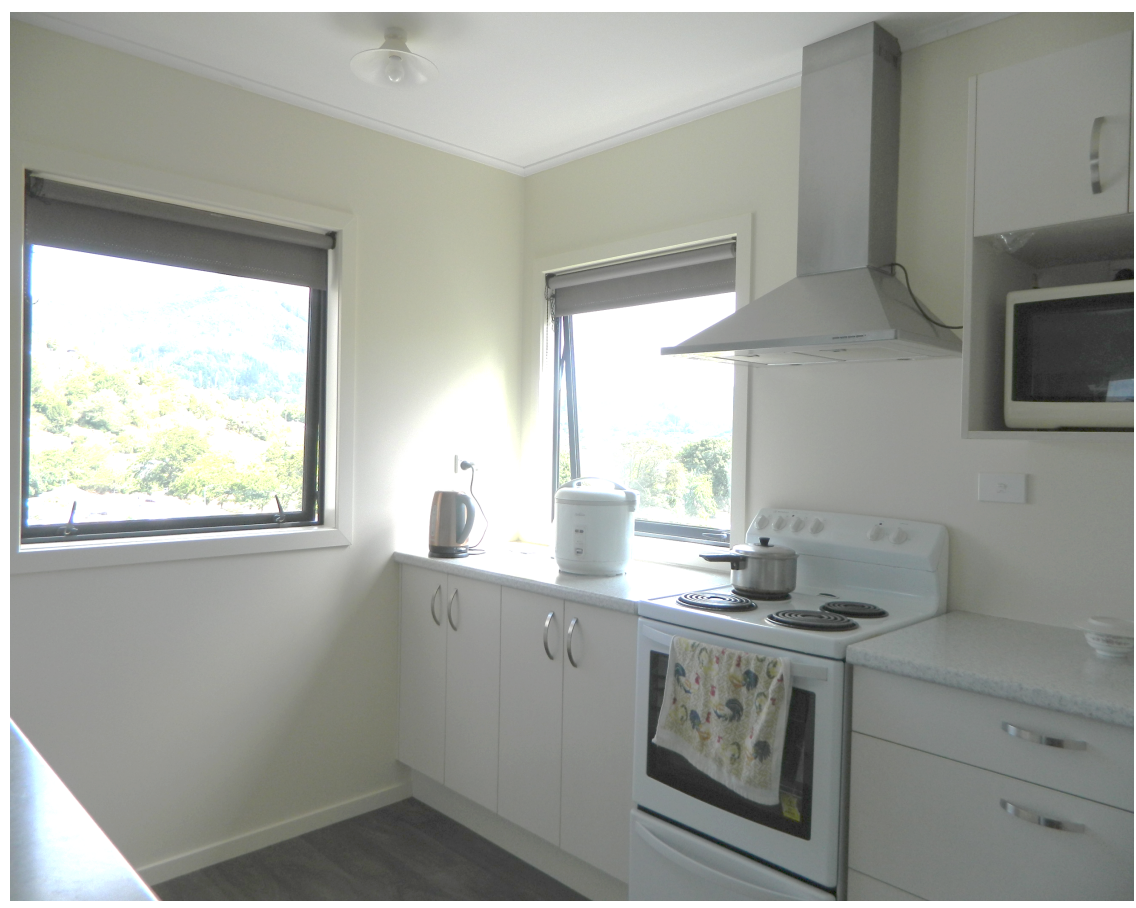

(Source: Photo supplied by the author, 2019) 
Several days later I returned to Toi Toi to interview 69 year old Ngun at her home. Ngun had only arrived a few months earlier, joining her husband, children, and grandchildren through the Family Reunification process and obtaining permanent residency. Ngun's daughter Dwat joined us to interpret and her five year old twin grandsons played music and ran around the living room whilst we talked. After sharing some fruit we walked out to the garden (Figure 4:10) to map the different stimuli, and Ngun and Dwat showed me the different herbs, vegetables, and flowers that they had planted.

47 year old Sui Sung also chose to do her interview at home so that she could show me her garden (Figure 4:11). Sui Sung had lived in Nelson for twelve years with her husband and children, coming to New Zealand as a quota refugee and later obtaining citizenship. We talked inside over coffee before wandering around the backyard to check out her vegetables. Sui Sung talked me through the different kinds of plants and showed me a poster she had on her kitchen wall with images of different vegetables and their English names. We then explored the front of the house where she had planted flowers and discussed her idea of having hanging flower baskets up the side of the fence.

After visiting Sui Sung I walked down the road to Iang Chin Sung's house. Iang Chin Sung was 34 years old and had lived in Nelson for the past six years. She had New Zealand citizenship, along with her partner and children, and had arrived through the annual UN refugee quota. She had previously worked at the Nelson fishing company Sealords during the Hoki season, though had to stop when her baby was born. She said that she felt attached to her home where she felt safe with her family (Figure 4:12), and we made coffee and talked in the kitchen whilst she fed her baby.

The following morning I interviewed Elizabeth at her home and explored her vegetable garden, tasting different plants as we walked (Figure 4:13). Elizabeth was 45 and had been in Nelson for longer than the others, arriving as a quota refugee fifteen years ago. She also had New Zealand citizenship, and lived in Toi Toi with her husband and children. She enjoyed spending time in her garden and walking up the hill behind her house to look at the view over the ocean. She spent a lot of time involved with her church and interpreting for other members of the Chin community. 
Figure 4:10: Ngun's Garden

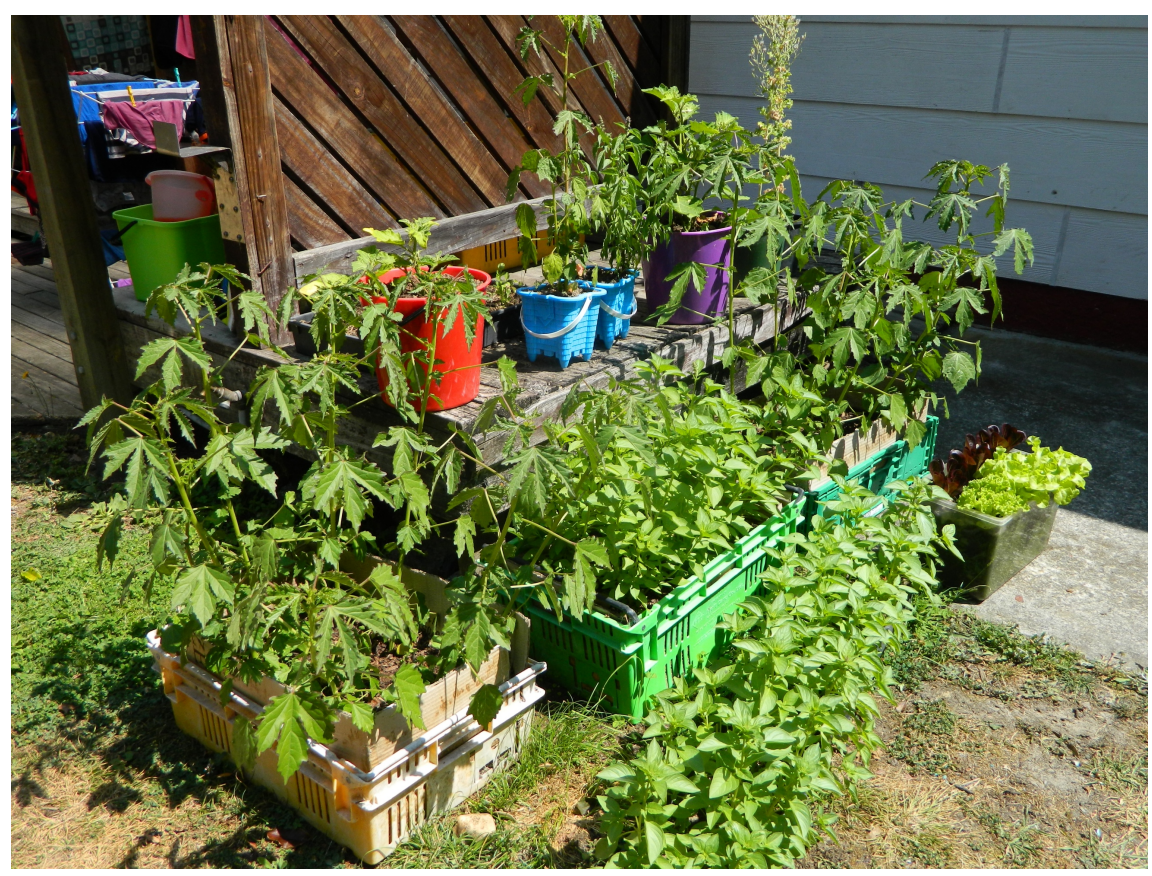

(Source: Photo supplied by the author, 2019)

Figure 4:11: Sui Sung's Garden

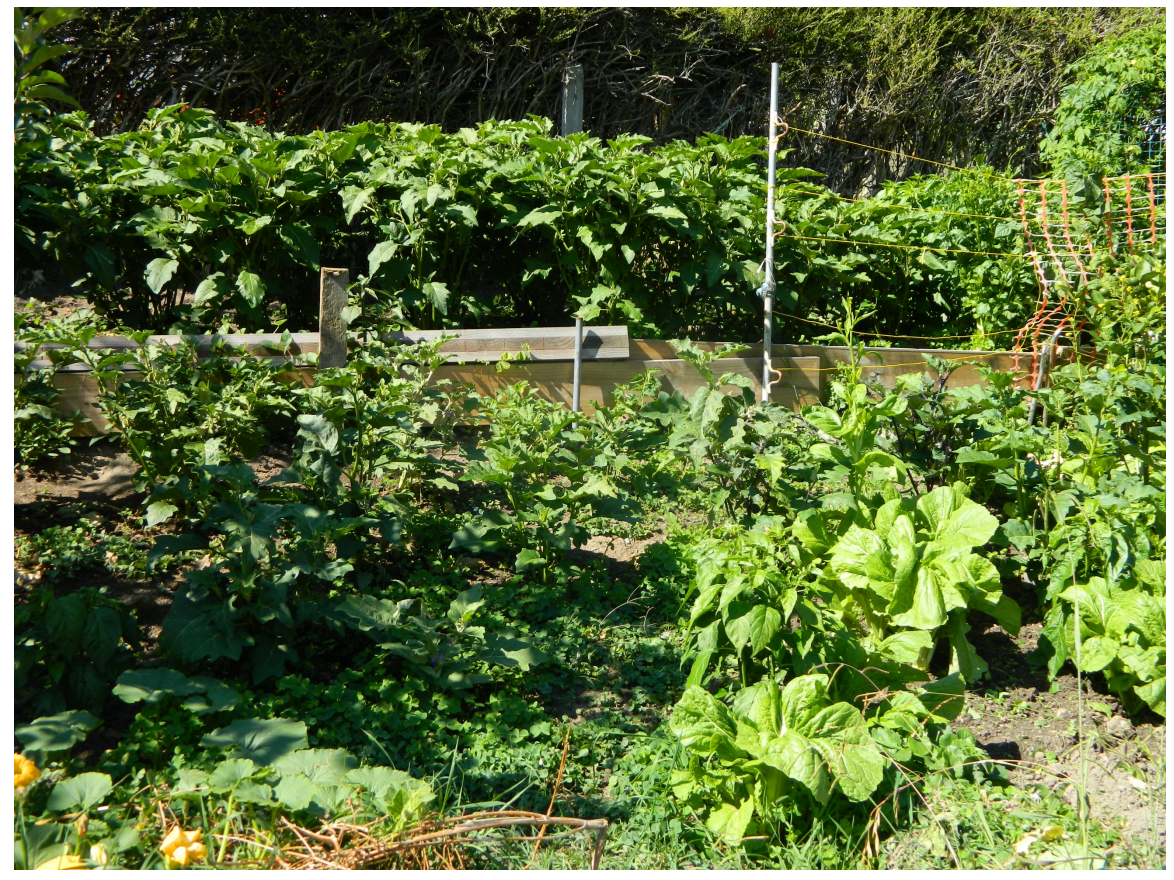

(Source: Photo supplied by the author, 2019) 
Figure 4:12: Iang Chin Sung's House

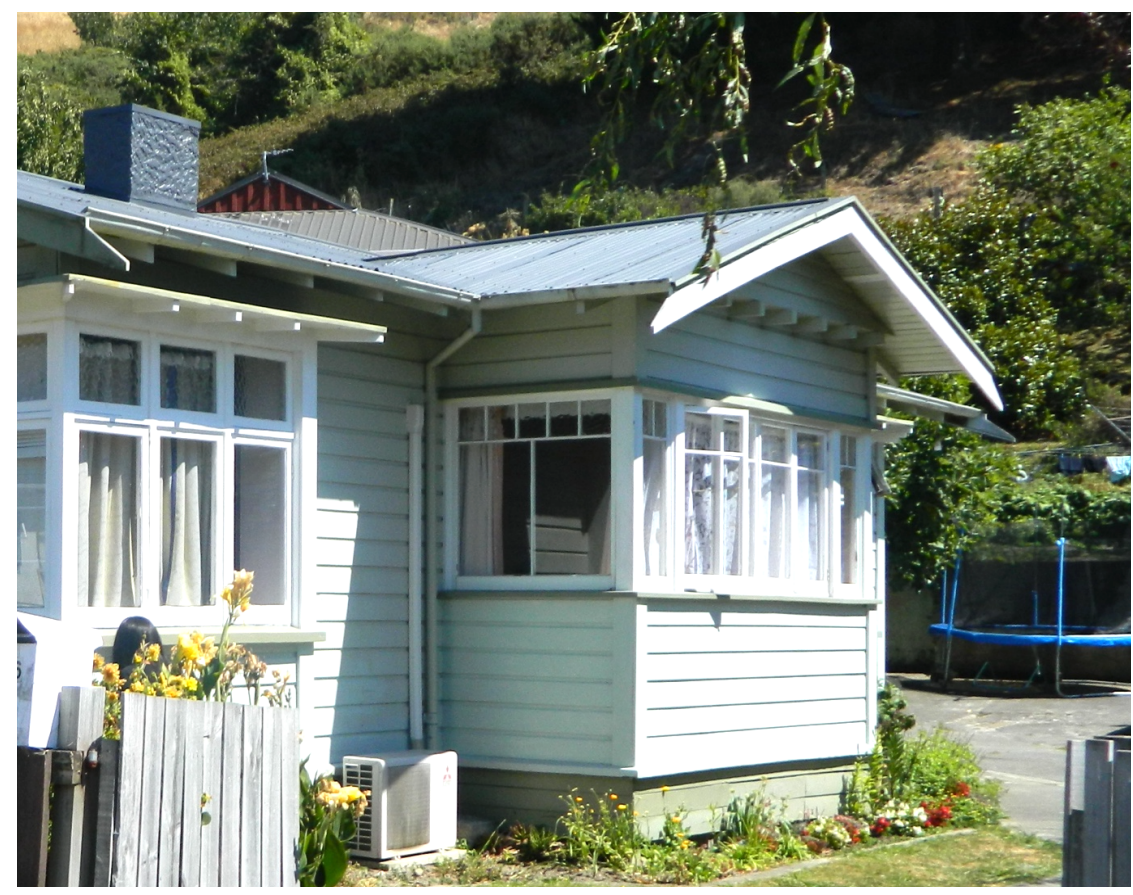

(Source: Photo supplied by the author, 2019)

Figure 4:13: Elizabeth's Garden

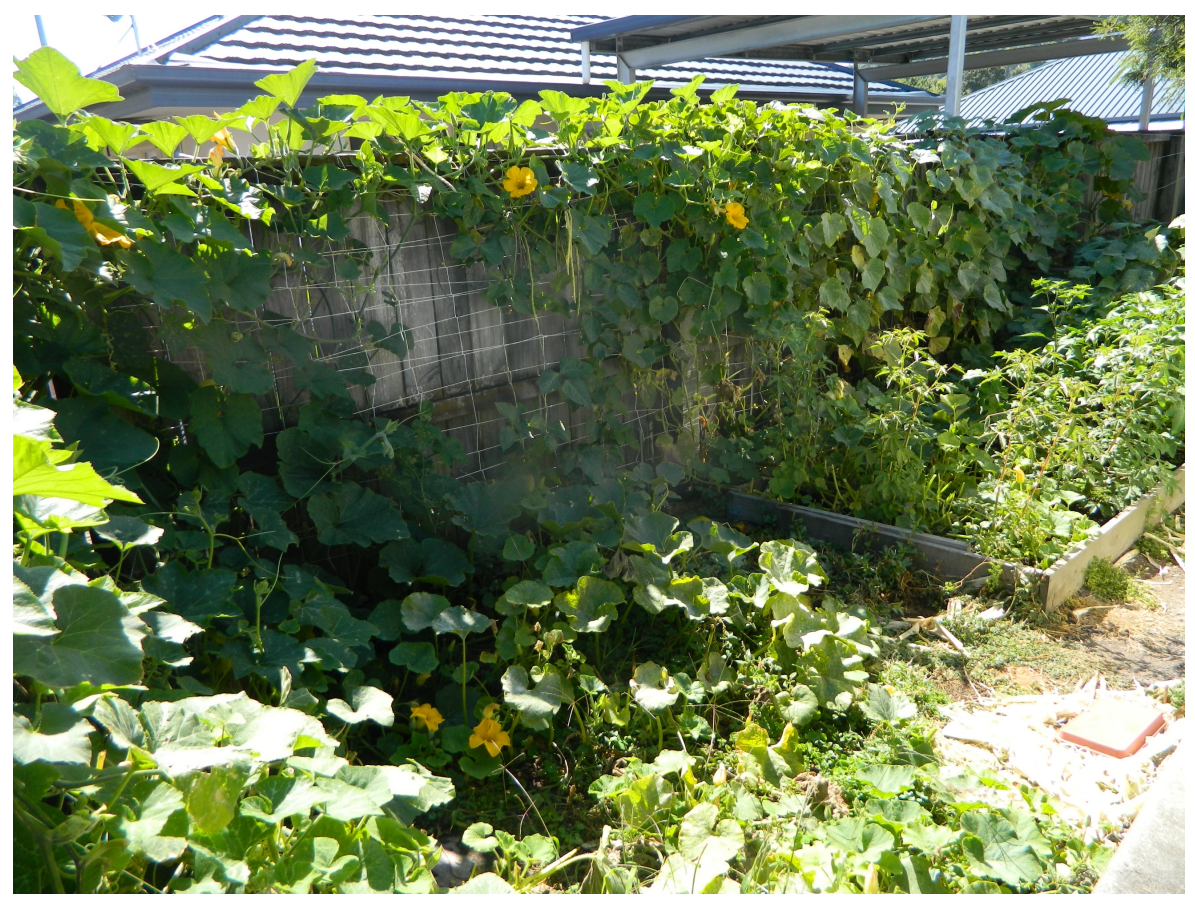

(Source: Photo supplied by the author, 2019) 
My next interview was with 34 year old Zai Zai at Broads Fields on the corner of Vanguard Street and Franklyn Street (Figure 4:14). Zai Zai had arrived in New Zealand as a quota refugee and lived in Nelson for nine years with her husband and children, all of whom now had New Zealand citizenship. We sat together under her favourite tree while we talked. Zai Zai enjoyed relaxing in the shade away from the busy city and said that the hills and the acorn tree reminded her of her home in Chin State. After we finished our mapping, Zai Zai dropped me off at her friend Jenavi’s house.

33 year old Jenavi had moved to New Zealand as a UN quota refugee a few years prior to Zai Zai, but originally came from the same rural village in Chin State. She lived in the Toi Toi area with her young children and had obtained New Zealand citizenship. A place to which she felt attached was her garden. Like many of the other Chin women, she had planted a mix of vegetables from New Zealand and Chin State. She also showed me her flowers (Figure 4:15), which she was worried about due to a strange texture growing on the leaves. To avoid the intense mid-day heat we did the interview inside. Jenavi gave me some of her favourite coffee to take home before driving me to Sui Tin Men's house.

37 year old Sui Tin Men came to Nelson as a quota refugee twelve years ago from the same village as Zai Zai and Jenavi. She has New Zealand citizenship and lives in her own home in Whakatu, Stoke with her husband and children. During our interview Sui Tin Men showed me around her garden (Figure 4:16) and together we discussed the negative aspects of spraying chemicals on the plants to remove the insects. She picked me a Chin-style cucumber from her garden to eat, slicing it up and showing me how she would prepare it with salt and chili and leave it in the refrigerator to cool.

Unfortunately, we could not visit all of the places that participants mentioned feeling attached to in Nelson. A major challenge in conducting site-specific interviews concerned the time, logistics, and resources involved in travelling to different locations. Many of the research participants were juggling study, work, and childcare and found it quicker, easier and more cost-effective to meet at their homes in between social events and school pickups. However, it became apparent that the sites which the girls and women had chosen were not only convenient, but also the places which they frequented regularly and where they had invested the most time and energy. 
Figure 4:14: Broads Fields

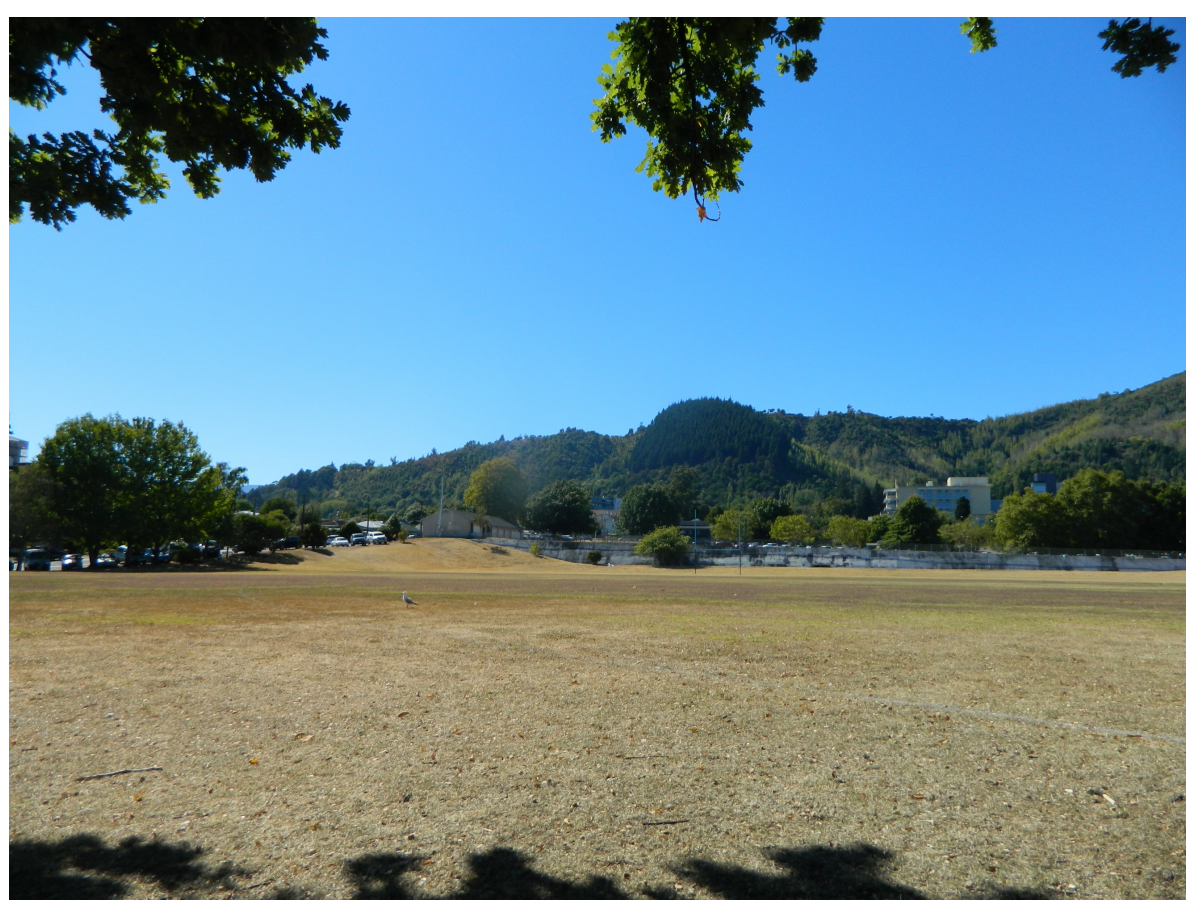

(Source: Photo supplied by the author, 2019)

Figure 4:15: Jenavi's Flowers

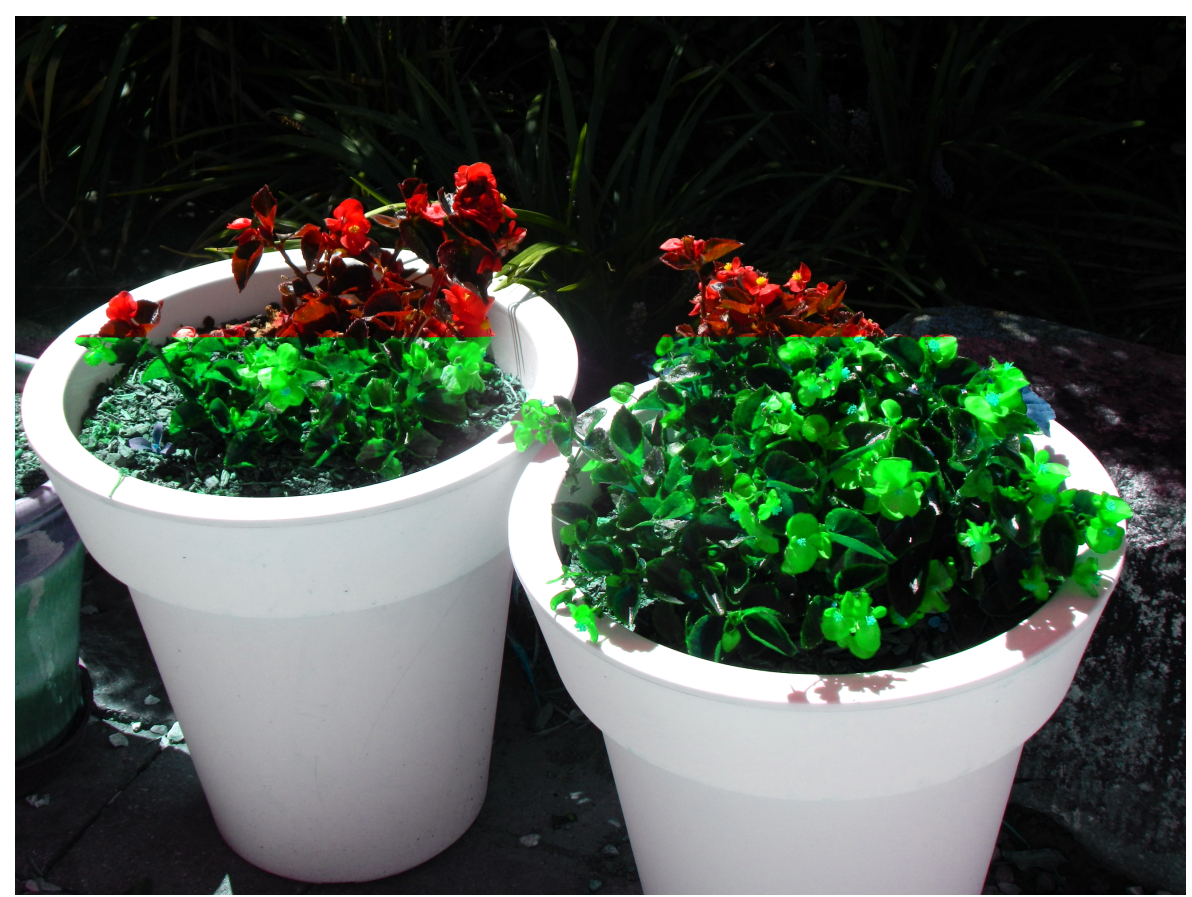

(Source: Photo supplied by the author, 2019) 


\section{Figure 4:16: Sui Tin Men's Garden}

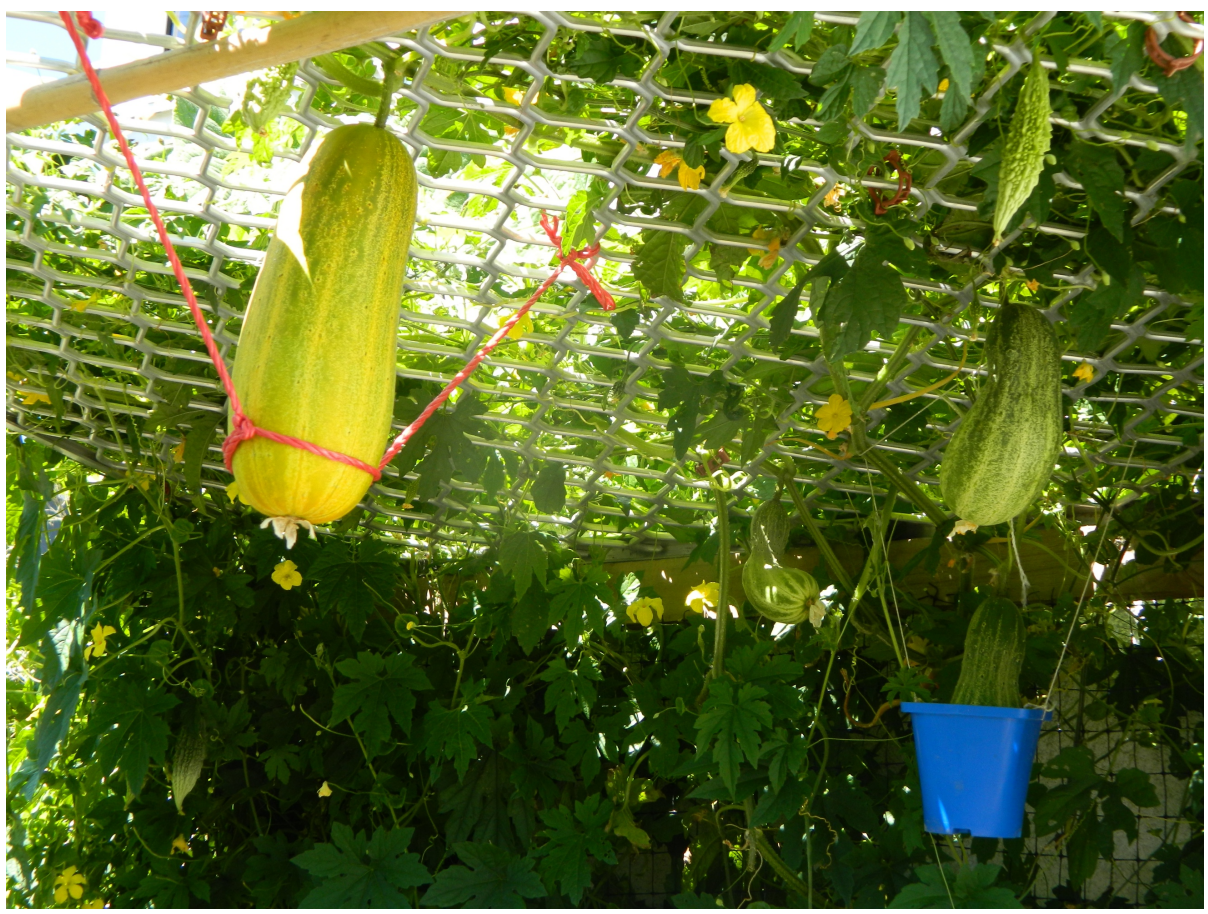

(Source: Photo supplied by the author, 2019)

Visiting these places together enabled me to become similarly situated to participants and step back from abstract representations to gain a more embodied intuitive understanding of their lived experiences (Pink, 2015; Thrift, 1996; Vannini, 2015). It also helped us to overcome language barriers. For example, when Sui Sung forgot the English word for 'coal' during her interview, she removed a handful of sooty black embers from her fireplace to show me the resource that they used for cooking in her village in Chin State. Reflecting on this interaction later, I wrote,

The soot stubbornly stuck to her hands, imbuing her gestures with the familiar smell of fire and demonstrating a tactile relationship with nature that was far removed from the experience of turning a plastic dial to ignite an electric oven. Watching her dust coal off her hands, I was reminded of countless summers barbequing food down the Marlborough Sounds, and became momentarily lost in a vision of mesmerizing flames dancing over an iron grill, the heat dispersing into the summer breeze and the smell of the smoke permeating clothing, hair, and skin (Fieldnotes, 11/02/2019). 
Several participants also faltered when trying to find the words to describe a specific flavour or sensation. Elizabeth led me out to her garden and handed me a torn green leaf with a warning to 'only take a small bite'.

Trusting Elizabeth's knowledge of edible plants, I tentatively bit the leaf. I immediately felt an uncomfortable tingling sensation on my tongue and lips, which built in intensity and left my mouth burning. I looked up to see Elizabeth grinning at me with a look that said, 'I did warn you', and despite a slight pang of regret I felt satisfied that, in this fiery visceral moment, we clearly understood one another (Fieldnotes, 12/02/2019).

Whilst these experiences did not teach me exactly what it felt like to be Sui Sung, Sui Tin Men, or Elizabeth, and whilst there was room for misinterpretation and the projection of my own emotions onto the women's experiences, these interactions were significant in building a new relational understanding and connection between us. They also highlighted the value of using the body as an instrument of research (Hahn, 2006; Longhurst et al., 2009; O’Neill \& Hubbard, 2010; Pink, 2015; Spinney, 2006; Tolia-Kelly, 2008).

The process of writing or drawing what they saw, heard, and felt on their body maps also prompted the girls and women to make new spatial connections which they hadn't raised during our oral interviews. For example, through the process of mapping the sounds in Jenavi's garden we came to discuss how the hum of the cicadas shaped her perception of time - specifically when to work and when to rest. Mapping the tastes and smells of Sui Tin Men's garden then prompted discussions around the use of pesticides and the ill visceral feeling this evoked during her pregnancy. Thus, the maps served their purpose well as a tool to facilitate deeper engagement with and awareness of time and place.

\section{Painting workshops}

After the interviews, I organised space and resources for painting workshops and invited participants along to paint their experiences to be displayed in a public art exhibition. The main workshop was held at the Victory Community Centre in Toi Toi (Figure 4:17), and facilitated as a group activity to create a relaxed, fun environment and enable participants to elicit new memories and ideas through shared practice and dialogue (Kale, 2018). Whilst I had considered working with Community Artworks, their venue was not as accessible as the community centre, which was situated within the neighbourhood where 
most participants lived and thus reduced travel time. The centre was also familiar to participants as a hub for refugee-background communities and well-equipped with public meeting spaces.

However, there were some participants who were unavailable at the time for which the workshop room was booked. Mu Pli joined me in a couple of earlier one-to-one painting workshops at the Victory Community Centre before she started her polytechnic course. To accommodate Aunty Ruth and Little Ruth, who lived in Stoke, I booked a space in the art rooms at Nayland College (Figure 4:18). This venue was selected as Little Ruth was preparing for her first year at Nayland and was nervous about starting a new school. Since the school was walking distance from her home, having use of the art rooms there meant that we could walk Little Ruth's school route together, and, being a former Nayland student myself, I could familiarise her with the different buildings before her first day.

\section{Figure 4:17: Painting at Victory Community Centre}

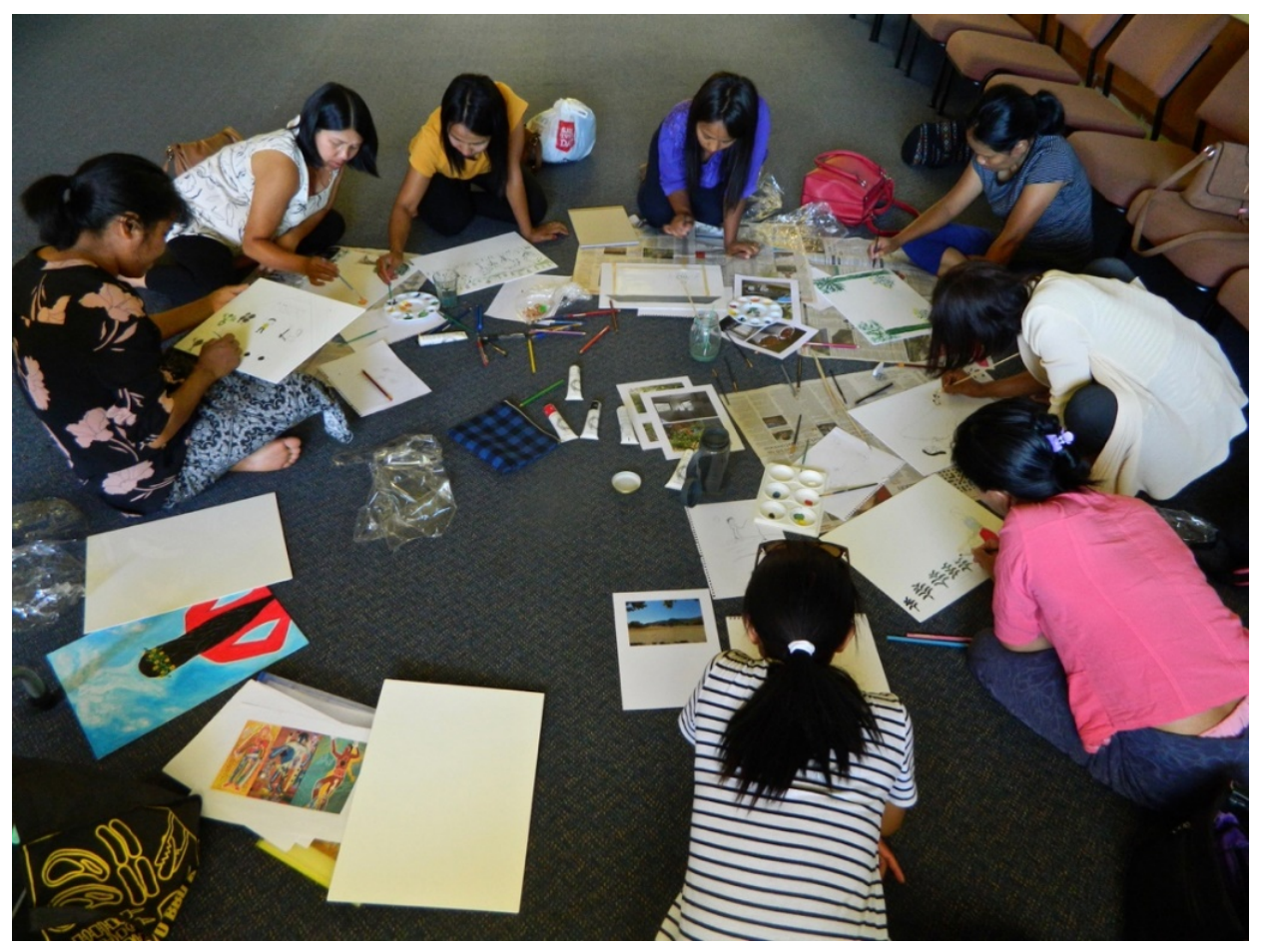

(Source: Photo supplied by the author, 2019) 


\section{Figure 4:18: Painting at Nayland College}

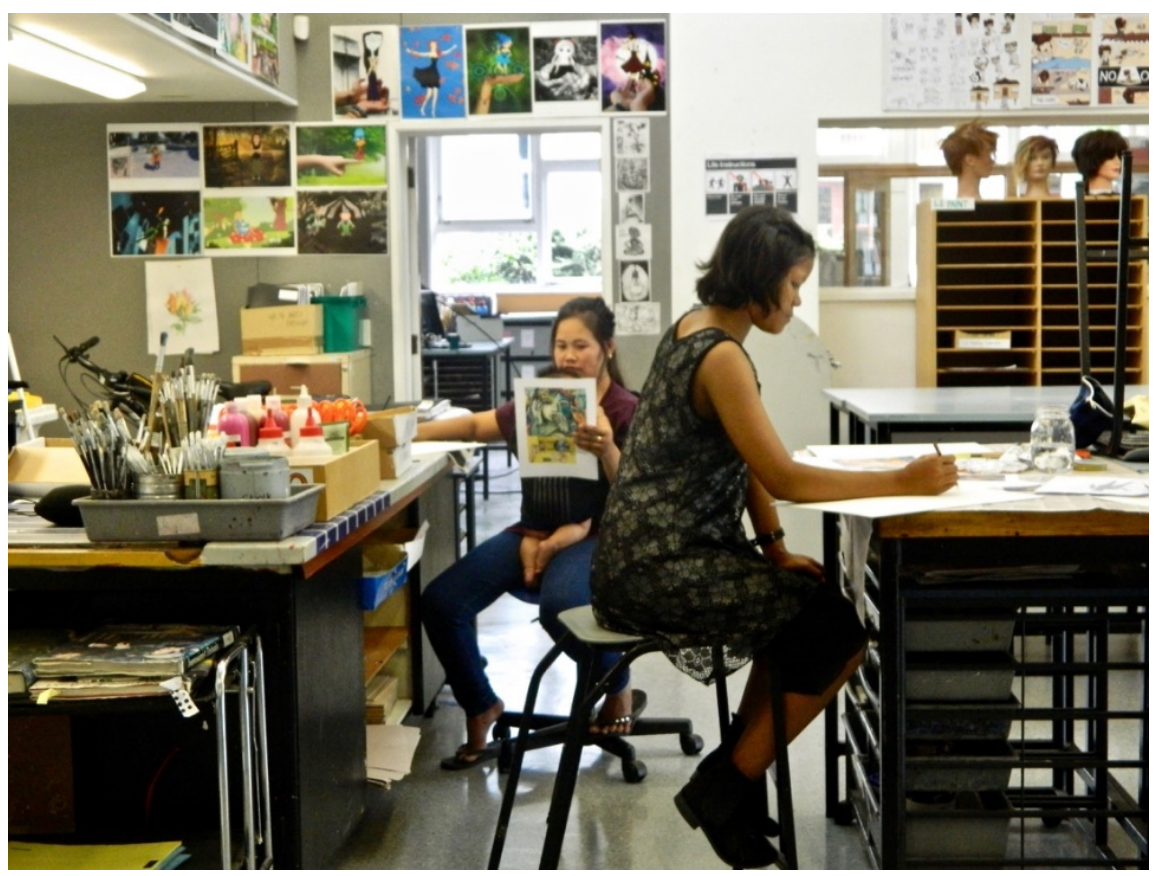

(Source: Photo supplied by the author, 2019)

As with the creation of the body maps, painting offered participants the time, space, and process for deeper reflection on their embodied relationships with Nelson. After choosing a specific place to illustrate, they decided upon the composition of the artwork and what specific aspects of place were important to include or leave out (Crang 2010; Kindon, 2016; Oldrup \& Carstensen 2012; Pink 2007). This process of composing or framing an image was significant in highlighting the nuanced ways in which participants perceived, used, and valued place, demonstrating their individuality and challenging dominant homogenising refugee discourses.

However, painting also came with technical challenges for participants and imposed its own representational limitations (Askins \& Pain, 2011; Kale, 2018). In particular, applying thick dark paint was scary as there was a concern that any mistakes would be permanent. To avoid this perceived problem, participants tended to thin down the paint, work with lighter colours, and tentatively apply their brush to the canvas to create a washed-out image that could easily be changed or painted over. For Little Ruth, this cautious approach resulted in her hair being painted an auburn colour rather than the dark brown she had mixed on the palate (see Figure 5:3). Yet, participants also said that they enjoyed the 
process of painting, and one woman asked if I might return to paint with her again someday - not for research but just for fun.

Using funding that I obtained through the 2018 Rosemary Seymour Award, I purchased supermarket vouchers for each of the participants and presented these vouchers at the end of the workshops as a koha - a gift to show my appreciation for the time and knowledge shared. I then printed participants' descriptions of their work onto plaques for the exhibition, framed the paintings, and confirmed the exhibition space and a time for an exhibition opening night.

\section{Collecting multisensory objects}

To enable the exhibition space to be multisensory and immersive, and to engage the audience in an embodied relational way, I also spent time collecting objects. I had initially hoped to collect these objects with participants during the interviews; however, half of the participants chose to paint places which we hadn't visited together, and items like flowers had to be purchased on the eve of the exhibition opening to ensure that they were bright and fresh. It would have been possible to bring participants together after the interviews and workshops to help me to collect these items; however, these tasks were arduous and time-consuming for the girls and women (who had already volunteered a significant amount of time to the project), and too costly for me to provide transport to re-visit interview sites or visit new places. For these reasons, I collected the objects and set up the exhibition on participants' behalf.

In addition to flowers we had seashells which connected participants to the smell of the ocean; spices imbued with the familiar scent of traditional Chin cuisine; a bowl of acorns for viewers to engage with Zai Zai's tactile experience of the acorns at Broads Field; and a miniature garden where viewers could plant seeds and get their hands in the dirt. Sound buzzers were also used to share native Myanmar birdsongs, bible readings, and church gospel music (Figure 4:19). Unfortunately, I couldn't access native bird calls or church proceedings in person so instead recorded short sound clips from publicly accessible (noncopyrighted) content online. The sound buzzers worked surprisingly well for the purpose of our exhibition, and several viewers specifically mentioned that they appreciated being able to hear the landscapes which were visually expressed in the paintings. 
Figure 4:19: Multisensory Exhibition Objects
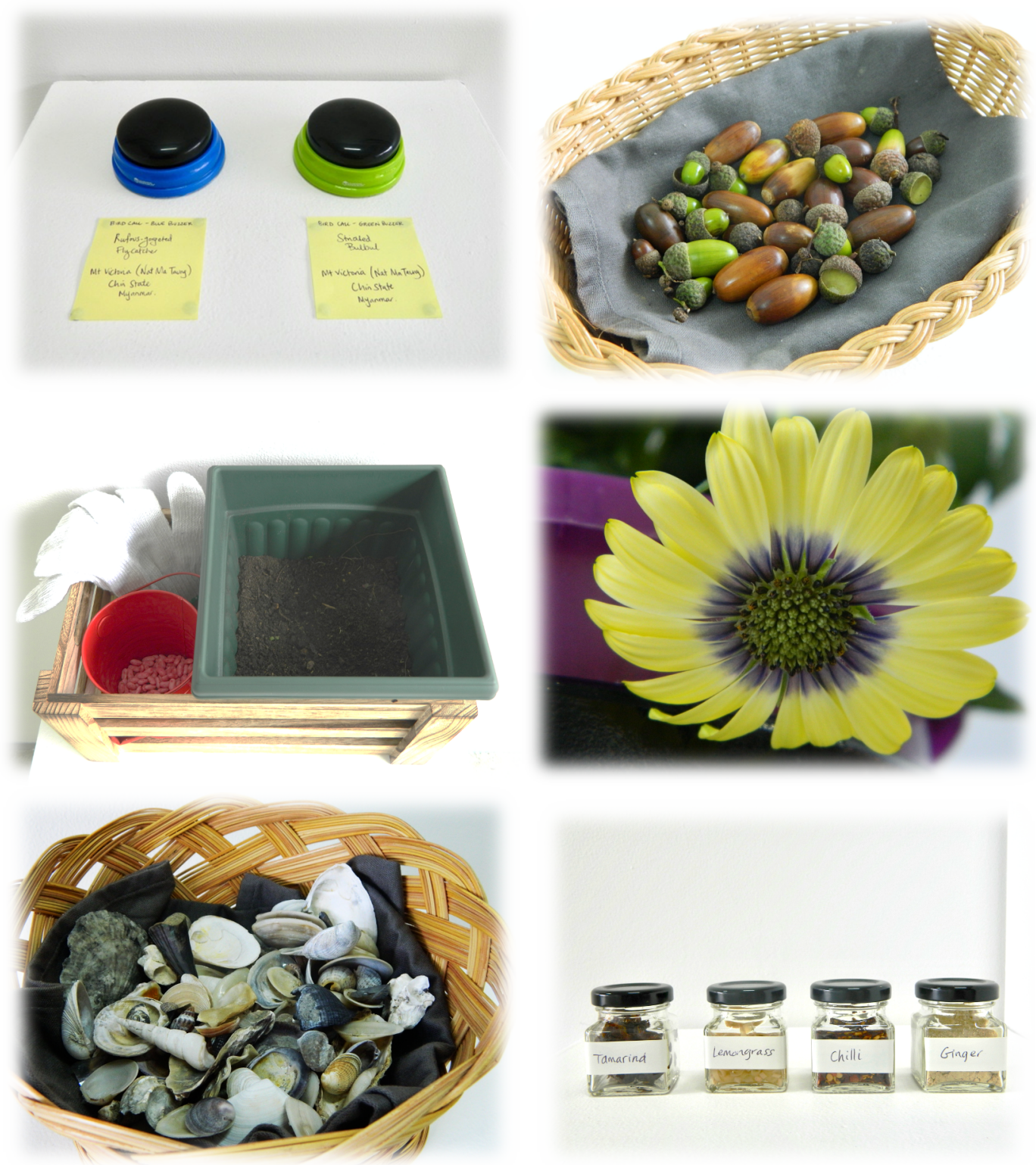

(Photos supplied by the author, 2019) 
In total, four months were spent on this second stage of fieldwork. Whilst this made for a rather compact, intensive study it helped me to build on public interest and maintain momentum. In this manner, the project took on a life and vibrancy of its own, drawing in a huge amount of local support and twice as many participants as I'd originally expected. However, the downside was having very little time in-between interviews and workshops for me to reflect on the processes and data being generated, and to rest.

To address these reflexive concerns, I set aside two hours an evening, three times a week, where I would walk up into the Ngawhatu hills behind my Nelson home and process my thoughts, shake off any worries, and find my own therapeutic-feeling place to re-energise. These walks became interwoven in my research process and the beautiful views, warm sunshine, and peaceful quiet of Ngawhatu inspired a unique understanding of the temporalities of place-attachment which later became a central strand of this thesis.

\section{The Multisensory exhibition}

In March 2019, I publicly exhibited participants' paintings and objects at the Nelson Marlborough Institute of Technology (NMIT) in their 'G-Space Gallery' in the CBD (Figure 4:20). The venue was offered to us free of charge, and NMIT helped us to advertise the exhibition to the public through the dissemination of a flyer (Appendix Nine). A reporter from the local newspaper also published an article about the research online and in-print to encourage Nelsonians to come and view the exhibition (O'Connell, 2019). On the first wall of the gallery, I displayed three posters with the research abstract, the collective street map showing the sites to which participants felt attached in Nelson, and the preliminary research findings (Appendix Nine). The framed paintings were then mounted on the walls and accompanied by plaques that described each participant's multisensory experience of place-attachment in their own words. Paintings were accompanied by the objects to share associated sounds, smells, and textures.

I then hosted an opening night celebration. Due to a terrorist attack against New Zealand's Muslim community only days earlier, I had some reservations about the timing of this event. Whilst participants were not Muslim, they were from a vulnerable minority group and many former refugees had been caught up in the attack and communities were hurting (Radio New Zealand, 2020). Other multicultural events around the country had been 
postponed for fear of further attacks, and I questioned whether raising the visibility of these girls and women might potentially expose them to undue harm. Yet, people around the country were also calling for social unity and positive multicultural stories, and we had worked hard to create a counternarrative to the fear, hatred, and discrimination explicit in the attack. We thus continued as planned, out of respect for those affected and in contribution to the strengthening of our multicultural communities.

Because there were no restaurants or caterers from Myanmar in the Nelson region, and the women themselves did not have the time or resources to cook for a crowd, we settled on Thai food, which offered a similar taste. I gave a short speech on the research themes and the multisensory mapping project. Participants were not expected to speak publicly (many of them were shy and lacking confidence in their English language abilities), and ethically they were not under any pressure to identify themselves as part of the project. However, they enjoyed speaking privately to members of the Nelson public (Figure 4:21), and a couple of the girls and women claimed that they felt proud of their artwork.

\section{Figure 4:20: The Exhibition at G-Space Gallery}

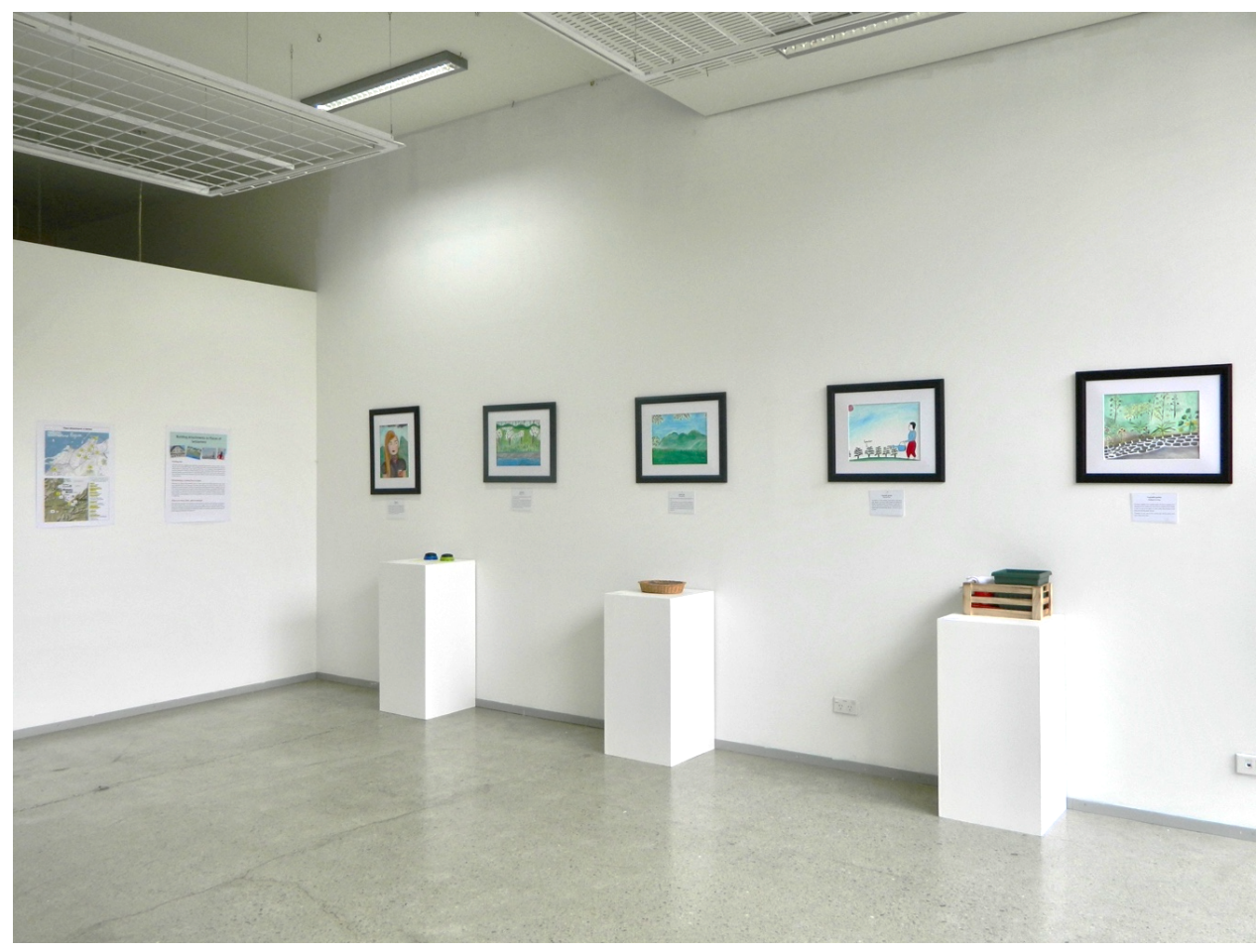

(Source: Photo supplied by the author, 2019) 


\section{Figure 4:21: Nelson Exhibition Opening Night}

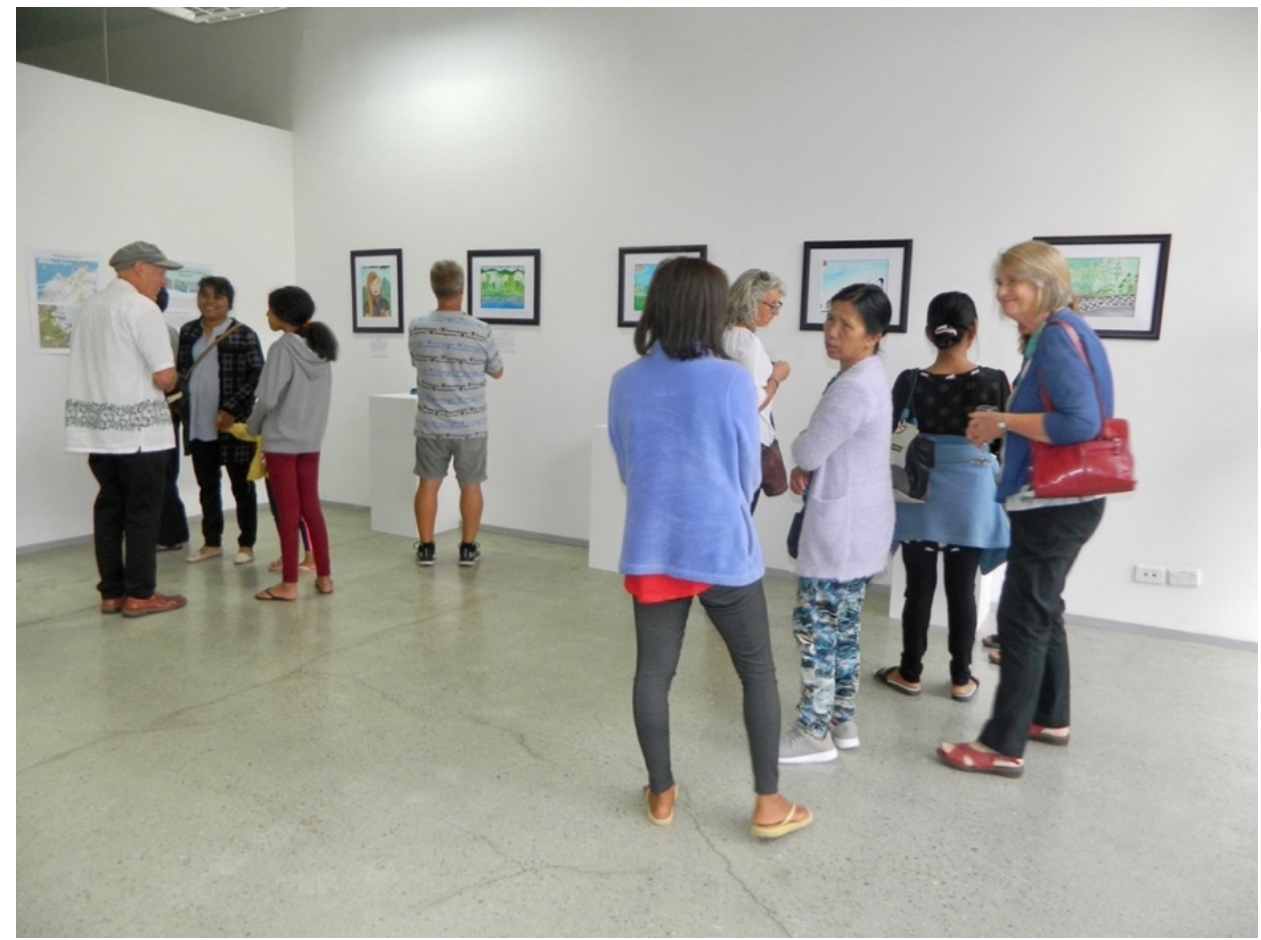

(Source: Photo supplied by the author, 2019)

This sense of pride was further evident through comments that participants made, saying things like, “The paintings look so good!", and their actions in pointing out their paintings to people viewing the exhibition and taking selfies beside their work. Hearing the womens' children exclaiming, "My mum painted this one!" further demonstrated the cumulative effect of pride being felt by those who were emotionally invested in the women's success and happiness. Elizabeth added that she was happy being viewed as an artist whose story was worth listening to, as opposed to being seen as a refugee, supporting the idea that participatory research can be empowering and transformative (mrs Kinpaisby, 2008).

For the exhibition audience, the paintings and objects raised awareness of the girls and womens' presence in Nelson and their involvement in local activities. Upon viewing the paintings and the place-attachment map, several Nelson residents said things like, "They [the women] go camping at Quinney's bush?! That's where I went camping as a child!', or, "Oh I'm glad they have been to Rabbit Island, it's great out there!" They were also smelling the spices on display and saying that they remembered travelling to new places 
themselves and being overwhelmed by foreign smells and flavours. Thus, whilst the diverse exhibition stimuli pulled into sharp relief the multiplicity of difference that is present in Nelson and the individuality of former refugees, it simultaneously evoked a feeling of commonality and a shared identity as Nelsonians.

The following day I facilitated a seminar for local NMIT students and members of the public who were interested in participatory arts-based research. The exhibition was then left on display for a week to reach more viewers, and a comment book was set up to record public feedback about the research project.

In November the exhibition was also displayed at the Dunedin City Library (Figure 4:22) as part of the 2019 Centre for Global Migrations Conference. I facilitated a public seminar at the library, speaking to the theme of refugee health and wellbeing. These events were followed by two further exhibitions of the paintings at Victoria University in Wellington, at the 'Learning Together in Aotearoa' Forum on December $6^{\text {th }}$ and $7^{\text {th }}$, and the University's first Diversity Festival on December 14 ${ }^{\text {th }}$ (Figure 4:23).

Figure 4:22: Exhibition at Dunedin City Library

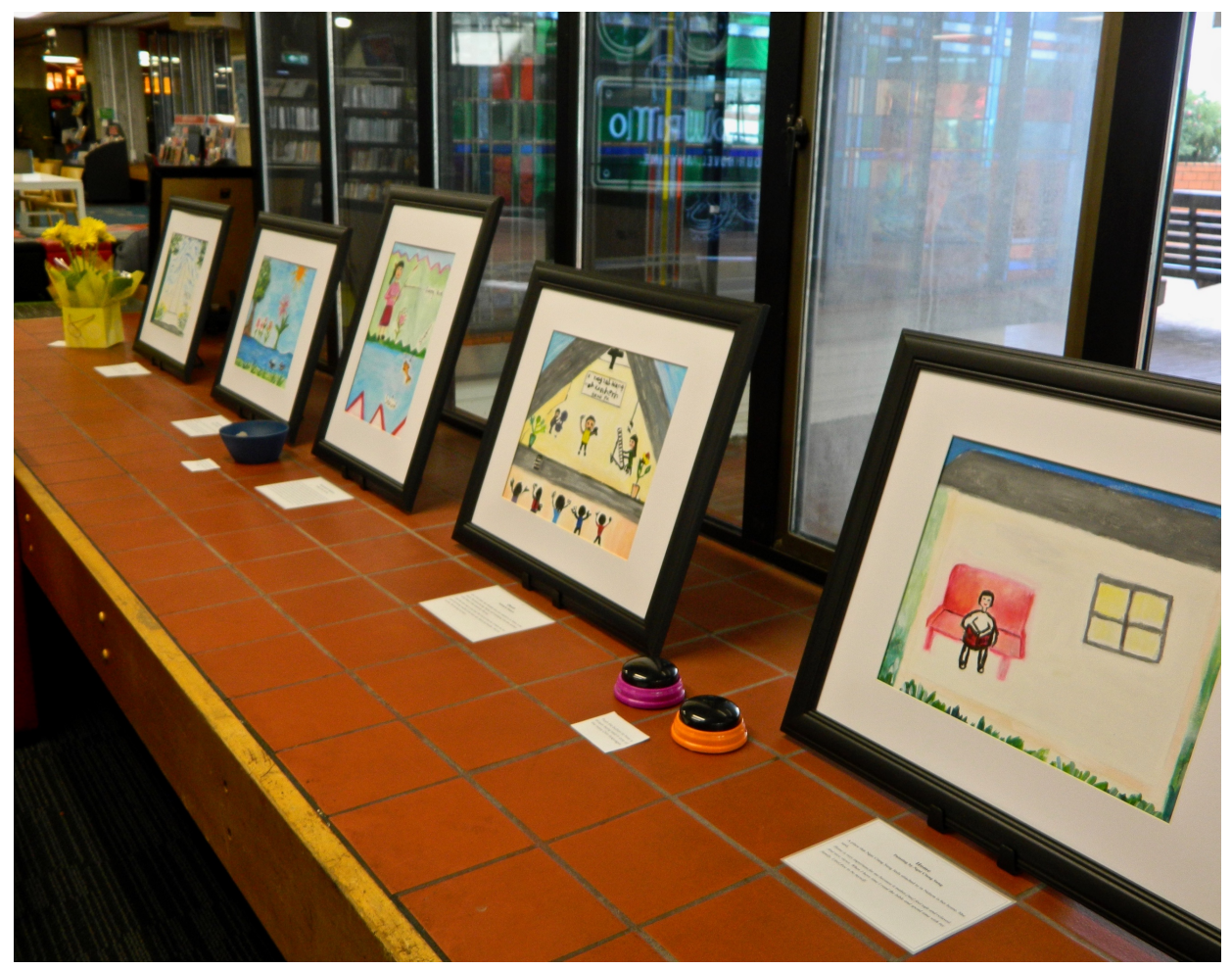

(Source: Photo supplied by the author, 2019) 
Figure 4:23: Wellington Forum and Diversity Festival Display

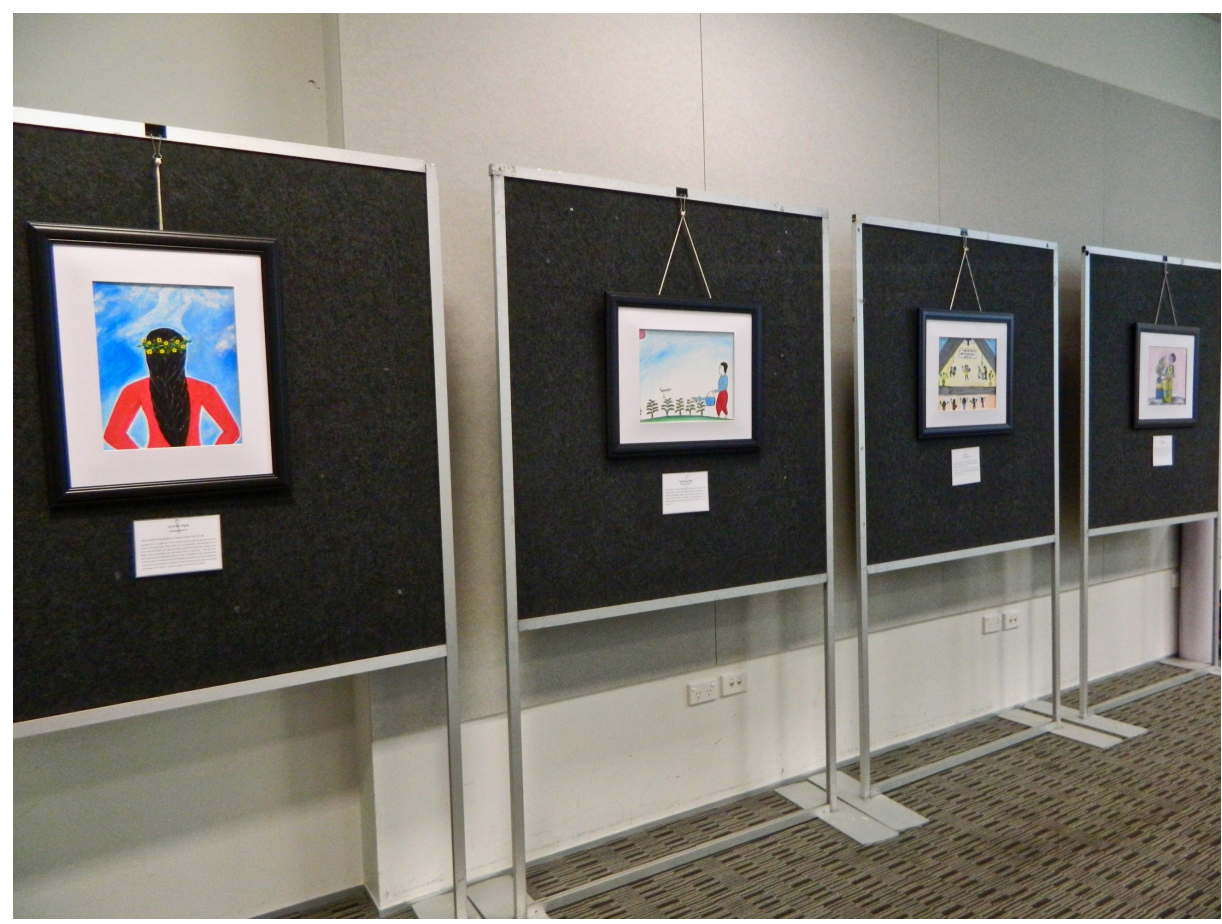

(Source: Photo supplied by the author, 2019)

Despite my careful curation, I failed to anticipate how the exhibition might change during its short time on display. In the Nelson gallery, the vibrant yellow flowers wilted in the evening, reviving themselves each morning when the sun streamed through the window. The green acorns turned various shades of ochre and burnt umber, and several of the seeds that viewers had planted in the miniature garden began to sprout beanstalks. On one occasion I had to change the lemon grass in the spice jars as it had developed a thin film of mould, and every time a breeze blew, I caught a faint whiff of the ocean coming from the seashells. In addition to these changes, the Dunedin exhibition was in a library which had its own dynamic sights, sounds, smells and events which viewers had to navigate.

This place agency was further evident as I transported the artwork and objects between different locations. In my reflections on the Dunedin exhibition I wrote,

Dragging a large suitcase of paintings down the street was no easy feat; not to mention the undignified debacle of heaving it up multiple hills whilst wearing a billowing dress. The bag was half my weight and at least half my height. The wheels were broken and I would drag the case for awhile, my arm and shoulder 
muscles straining, then stop and push it, bruising my knees as I stumbled over cracks in the concrete. In the hot weather I began to sweat, cursing the decision to facilitate an art project instead of simply conducting interviews. As the humdrum of the city ebbed and flowed in and out of my thoughts, the soundscape was disrupted by the loud melody of Chin gospel music - coming from one of the sound buzzers in my backpack. Trying desperately to turn it off, I knocked the spice jars that were to accompany Ruth's painting of her kitchen, and wound up covered in flakes of chili and smelling like ginger. By this stage I was unsure if I was facilitating an exhibition or part of it; parading a unique array of unfamiliar sights, sounds, and smells through the city (Fieldnotes, 03/12/2019).

These evolving circumstances and material interactions served as a reminder that research emerges through a dynamic emplaced relationship which is inescapable from the events and rhythms of everyday life (Lefebrvre, 2004; Pink, 2015, Potts, 2015; Seamon, 1980). Reflecting back on the aforementioned need for temporal sensitivity in participatory practice, it is important to question how research is impacted by daily and seasonal cycles. Furthermore, how do people, events, and conversations flow? What happens when researchers stumble, get stuck, and experience fatigue? (Marston et al., 2005; Thrift, 2008), What habits, routines and rhythms are being co-produced (Edensor, 2010; Seamon, 1980), or disrupted through research practice? And what implications do these affective temporalities have upon research processes and outcomes? I continue to reflect upon these questions throughout this thesis.

\section{4:3 Data collation, analysis, and dissemination}

\section{Transcription, collation, and analysis}

Upon completion of the interviews and workshops, audio-recorded data was transcribed verbatim. A 'Codebook' in the form of a matrix (Appendix Ten) was used to distil and synthesise the transcribed data and identify key themes (Cope, 2016). Three types of codes were used, consisting of manifest 'in vivo' codes - identifying the terms and expressions commonly referenced by participants; descriptive codes - which expand on in vivo codes to explore obvious themes and patterns concerning who, what, where, when, and how; and 
latent analytic codes - chosen to link descriptive codes to existing ideas and theories for the purpose of contextualising the data in a wider conceptual framework (Cope, 2016).

Data from the practitioners scoping interviews was organised into three categories consisting of social environment, physical environment, and wellbeing. These particular categories were established to understand the types of place-based experiences that former refugees have in Nelson, and the types of services available to support them through these experiences and enhance their wellbeing. Data from the multisensory mapping project (including my reflexive writing based upon my own research experiences and the notes that participants had made on their maps) was then organised into five categories. These categories consisted of 'phenomenological experiences' (such as sensory perceptions, memories and emotions), 'social experiences' (including the ways that social norms and cultural imaginings shape sensory experience), 'technological experiences' (concerning the role of mobile communication technologies in shaping place-based experience), 'environmental experiences' (looking at sensory place characteristics such as terrain and climate), and wellbeing (detailing feelings of health and wellness in place). These categories enabled deeper explorations of the people, place, and process dimensions outlined in environmental psychologists Scannell and Gifford's (2010) Tripartite Model and my emplaced model of place-attachment and wellbeing in Chapter Three.

To collate and analyse the visual images from participants' maps and paintings, I took a similar approach to the earlier textual thematic analysis, examining the artwork and noting key themes. I then linked these notes back to the coded interview transcripts and research reflections. In this manner, I was able to connect Mu Pli's symbolism of yellow flowers in her painting of ANZAC Park with her interview discussion around feeling intrigued by the flowers, and my own experience of seeing these flowers at the park when we visited together. These visual, oral, and experiential forms of data generation were then woven together in my coding matrix through the emerging concepts of 'beauty', 'enchantment', and 'happiness'. Thus, thematic visual analysis was partly guided by my own interpretation of the images, but also heavily influenced by participants' prior discussions on what was significant to them in the places they had painted, and their actions in attuning my attention to specific details during our interviews. 
Connecting the imagery to our earlier discussions and experiences in this way was important, as Sociologists Oh (2012), and Oldrup and geographers Carstensen (2012), and Rose (2012) have claimed that oral and textual discourses are necessary to contextualise imagery within a specific time, place, and culture and enable insight into the social significance of visual content. Additionally, I suggest that this process also enabled insight into the more-than-human significance of visual content.

Because I did not assume specific senses would be discussed, or aim to separate the senses, the coding process also provided scope to move beyond a Western five sense framework to include more visceral and proprioceptive experience and to consider how the different senses might be interwoven (Bodhi, 2000; Champagne, 2004; Steiner, 1981). This approach was significant as it led to an understanding of the different atmospheres and rhythms of place, which are comprised of multiple overlapping senses and movements, and enabled me to revise Scannell and Gifford's (2010) model with a greater emphasis on the temporalities of attachment. I then analysed the coded data through my emplaced conceptual framework, considering the spatiotemporal, sociocultural, and more-thanhuman influences which shaped participants' (and my own) embodied research experiences, interviews, maps, and paintings.

\section{Dissemination}

In addition to the community art exhibitions, I disseminated my research at various academic events. Early and ongoing academic dissemination was important to ensure research rigour and enhance credibility and trustworthiness - opening up ideas for scrutiny and feedback (Bradshaw \& Stratford, 2010). On a 2018 trip to the UK, my supervisor Professor Sara Kindon and I co-presented a seminar at the University of Glasgow in Scotland at one of their academic 'Testing Grounds' sessions. We also gave a joint presentation at Northumbria University in Newcastle England, before joining my second supervisor Dr. Polly Stupples and co-convening three sessions at the 2018 Royal Geographical Society with the Institute of British Geographers Conference (RGS-IBG) in Cardiff, Wales. I presented two papers at the RGS-IBG conference, one of which I later published in the Journal of Environmental Psychology (Kale, 2019). Sara and I then 
recorded a Podcast about our individual research projects for the RGS 'Ask a Geographer' series (Royal Geographical Society with IBG, 2018).

Following the RGS-IBG conference, I attended a residency with the Visual Research Network (VRN) in Brighouse, England, and presented a paper at their 2018 conference in Manchester. I then returned to New Zealand to share my RGS-IBG paper with the Nelson resettlement practitioners, to offer them the opportunity to engage with any my analysis and make any changes or recommendations before I integrated this work into my thesis. I also presented this paper at the 2018 Otago University Centre for Global Migrations Conference in Dunedin, which was attended by resettlement researchers, practitioners, and former refugees.

In a second trip to the UK in 2019, I presented a paper at the UNESCO RILA Spring School in Glasgow, Scotland. Along with Dr. Melike Peterson from the University of Bremen in Germany, I then co-convened two sessions at the 2019 RGS-IBG Conference in London. I acted as chair at one of the sessions and presented a paper. I also became involved in organising and hosting the second annual Visual Research Network (VRN) residency and conference in Manchester in September.

On my return journey to New Zealand, I connected with a development organisation in Chiang Mai Thailand, who work with refugees (predominantly from the Karen and Shan communities) who are fleeing across the Thai / Myanmar border. Through this organisation I visited migrant communities living on the outskirts of Mae Sot, and refugees living in Mae La Refugee Camp. This experience was not officially part of my research, and thus I did not apply for ethical clearance or conduct any formal interviews. However, my experiences offered me valuable insight into the broader political context in which my research is situated, and a greater understanding of the journey that many Myanmar refugees courageously embark upon en route to New Zealand. I therefore make reference to this trip throughout my thesis, though refrain from using any direct quotes, names, or photographs.

At the start of 2020 I then worked with the Victoria University communications team to produce a promotional research video to generate further interest in my research (Victoria University of Wellington, 2020). Unfortunately, other planned events for 2020, including 
the American Association of Geographers (AAG) Annual Meeting in Denver, Colorado, and a final re-engagement with research participants in Nelson were cancelled due to the novel coronavirus and subsequent national lockdown in New Zealand. This community reengagement is important to enable research findings to be applied in practice, and I hope to still pursue this at a later date.

\section{4:4 Key learnings}

Because people, places, and relationships are dynamic and complex, I agree with anthropologist Pink (2015) that there should not be a set methodological template for doing multisensory research. However, the participatory arts-based approach and unique methods explored in this chapter were valuable, and may be useful to alter or build upon in other research contexts. Of particular significance was being together with participants in the specific environments which we were discussing. These emplaced interactions enabled us to move beyond metaphorical language to communicate in a multidimensional way, using the materiality of bodies and objects in time and space to intuitively understand complex (inter)subjective experiences (Longhurst et al., 2009; MacDougall, 2005; Pink, 2015). Such immersive research also enabled us to challenge vision as a privileged vehicle of knowing in Western epistemologies (Trnka et al., 2013), and explore the multisensory events, rhythms, and experiences which shape everyday life.

Bringing three-dimensional objects into the exhibition space further supported this shift beyond the visual, attuning the exhibition viewers to the sounds, smells, and textures which had become an important part of place-making and home-making for the girls and women as they resettled in Nelson. However, these basic exploratory methods could be expanded upon, and I provide suggestions for future multisensory exhibitions in Chapter Nine.

It is important to clarify that moving 'beyond the visual' does not mean omitting that which is seen, and the practices of map-making and painting in this research helped to further attune myself and participants to our surroundings and prompt deeper spatiotemporal engagement and reflection. The paintings also offered a unique way to disseminate the research beyond the academic sphere and share participants' experiences 
with the wider Nelson community, weaving their stories into a wider spatiotemporal narrative and challenging the notion of refugees as unfamiliar or threatening 'outsiders'. Two other salient points which emerged through this research process were the need for temporal sensitivity in participatory research (mrs kinpaisby, 2008), and greater sensitivity to non-human entities - including the tools and objects that are used within research practice (Askins \& Pain, 2011), but also the food that is shared (Longhurst et al., 2009), and the terrain, climate, flora, and fauna which may guide research in nuanced and unpredictable ways, disrupt plans, or support particular ways of co-constructing and disseminating knowledge (Bawaka Country et al., 2016).

Because participatory research is inextricably linked to these wider spatiotemporal relationships, events, and rhythms, there is an inherent messiness to it that, to some degree, researchers need to take in their stride. Having a relatively open and fluid methodology that embraces multiple realities and recognises moments of rupture and surprise as part of the research process (Pain \& Kindon, 2007) is possibly both the greatest asset, and the greatest challenge, in co-producing scientifically rigorous and emotionally and socially meaningful research. 


\section{Chapter 5: Sensory Memory and the (Re)Creation of Home}

Building upon the data generated through participants' interviews, maps, and paintings, I discuss the research findings in the following chapters, beginning with a focus on sensory memories and practices of homemaking. Participants commonly claimed that they felt drawn to specific multisensory environments in Nelson which evoked positive memories of their former homes and helped them to feel emotionally connected to the places where they grew up. These nostalgic experiences enabled them to feel safe and relaxed in a new place and to maintain a sense of self-identity. However, they also prompted feelings of homesickness and a sense of being out-of-sync with one's surroundings. Loud sounds, strong smells and movements, and particular visceral sensations could also trigger violent and fearful memories of home and exacerbate resettlement challenges. Exploring this ambivalence between the past, present, and future, I argue for a more fluid temporal approach to place-attachment and wellbeing, which enables individuals to mediate between different times and places. I then draw upon geographer Blunt's (2003) concept of 'productive nostalgia' to understand how the girls and women are actively (re)creating feelings of homeliness and regaining a sense of safety, stability, and happiness in Nelson.

\section{5:1 Feelings of nostalgia}

\section{Remembering home}

For the Chin and Kayan participants, the experience of moving to New Zealand and becoming attached to new places was entangled in memories of distant homelands and personal understandings of what home is, what it feels like, and what it should be. To provide insight into the diverse places which individual participants were remembering as 'home', I begin this chapter with a short introduction to the places in Myanmar to which participants are historically connected, and which affected their sense of place in Nelson. I also introduce other places in which participants made homes en route to New Zealand.

Myanmar is a Southeast Asian nation bordering India, Bangladesh, China, Laos, and Thailand (see Figure 5:1). Archaeological evidence shows that humans inhabited this 
region as early as 11,000 BCE (Cooler, 2002), though the country's modern formation can be traced to the rise of the Pagan Empire in the late $12^{\text {th }}$ century (Harvey, 1925). Modern day Myanmar has a population of 51 million, comprised of over 140 distinct ethnic groups. Of these groups, 135 are officially recognised by the Bamar (Burmese) government and are divided into eight main ethnicities named Bamar, Chin, Kachin, Kayin (Karen), Kayah, Mon, Rakhine, and Shan (Oxford Burma Alliance, 2018). Myanmar is also home to the ethnic Rohingya; yet, despite centuries of Rohingya habitation in the state of Rakhine, the Bamar government does not recognise Rohingya as citizens and this group is considered by the UN to be 'stateless' (Oxford Burma Alliance, 2018). Other unrecognised minorities consist of immigrant populations from India, China, Nepal, and Europe (Smith, 1994). These distinctive ethnic groupings and politics are reflected geographically. The country is divided into fourteen different territories, seven of which are referred to as 'regions' and are predominantly inhabited by the dominant Bamar (who make up roughly two thirds of the total population). The remaining seven territories or 'states' are divided amongst and named after the seven other official ethnic groups (Oxford Burma Alliance, 2018).

Of the twelve research participants, eleven identified themselves as 'Chin'. The Chin people are of Sino-Tibetan origin and are composed of a number of sub-ethnic groups including the Asho, Cho, Khumi, Kuki, Laimi, Lushai, and majority Zomi group. For centuries they have inhabited the mountain ranges in Myanmar's Western Chin State, commonly referred to as Chin Hills (Minority Rights Group International, 2018). The capital city, Hakha, is situated at an altitude approximately $6000 \mathrm{ft}$. above sea level. Here, the hot summers, mild winters, and rainy seasons reflect the warm tropical monsoon climate that is typical of Myanmar, although the high altitude can also bring cooler temperatures than are experienced in the lower regions (Scarlis, 2010). Residents are primarily involved in agriculture, farming, and freshwater fishing (Frissard \& Pitts, 2018).

The New Zealand city of Nelson, where participants have resettled, is also a mountainous agricultural region, with forests, farmland, fisheries, vineyards, and access to rugged bushland (New Zealand Trade and Enterprise, 2016). Whilst this region has a cooler temperate oceanic climate than Southeast Asia, the city is situated on the coast at a lower altitude than Chin State and has the most sunshine hours in New Zealand (NIWA, 2018). 
Figure 5:1: Ethnic States and Regions of Myanmar

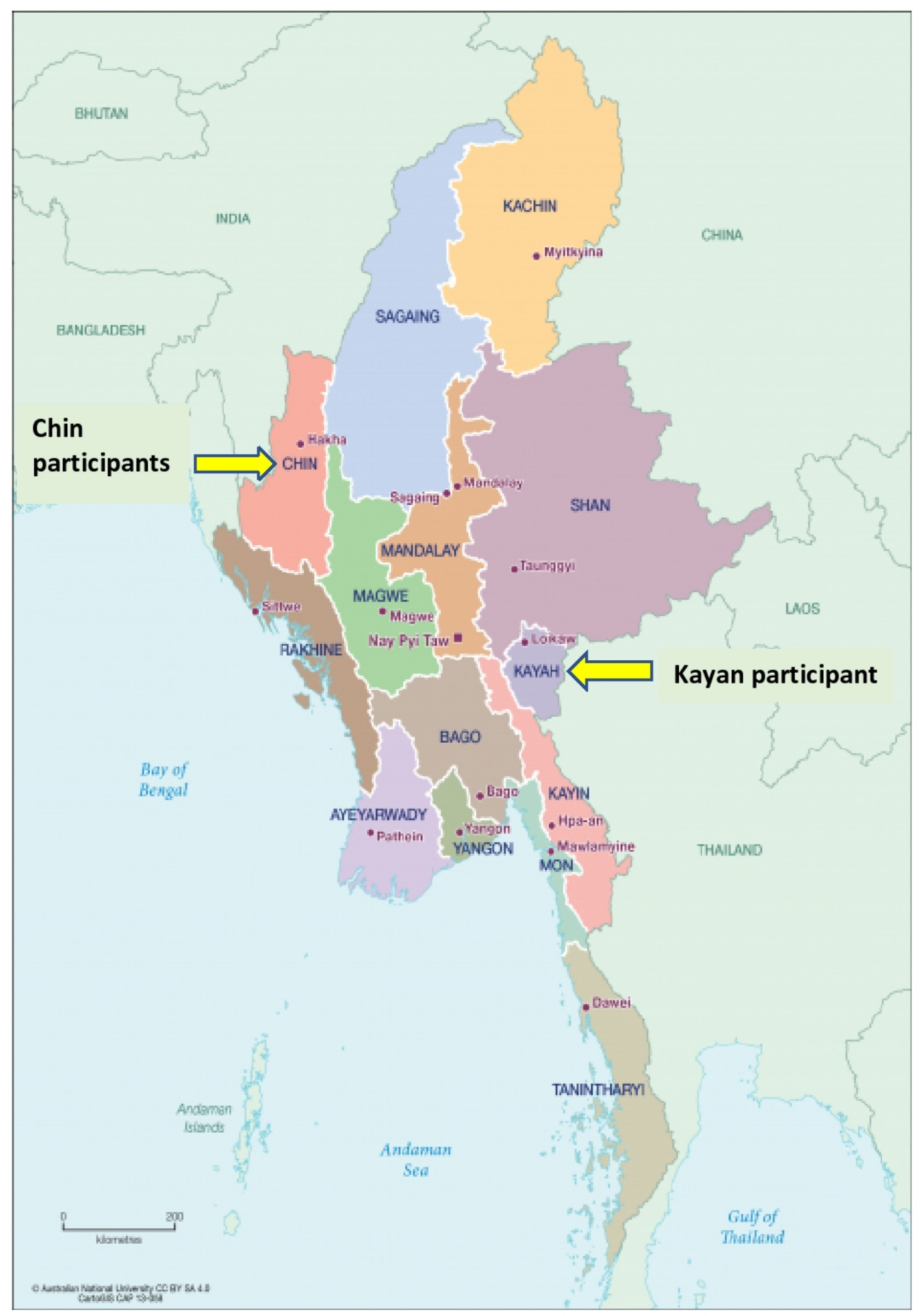

(Source: Australian National University, 2019) 
In her interview, Iang Chin Sung said, "I am from Chin State and the weather there is very similar to the weather in Nelson. The mountains are also similar". One of the resettlement practitioners said, "One of the reasons that Nelson was chosen as a place for Burmese is that those particular people have come from the hill areas and there is a similarity". On her street map, Ngai Ciang Sung wrote, "I like Nelson because it reminds me of Chin State. The houses, mountain, people are nice". Sui Tin Men said, "Sometimes we go to the Queens Gardens [a botanic park in Nelson] ... I feed the birds and the fish, it reminds me of Chin State" (Figure 5:2). Little Ruth added that she enjoyed walking up the mountains, seeing the flowers, and listening to the birds at Whenua Iti (a youth development course in Lower Moutere, a 40-minute drive from Nelson City) (Figure 5:3). She had completed a course here the week before I interviewed her and claimed that, whilst the birds in New Zealand sound different from the birds in Myanmar, the mountains reminded her of time spent with her family back home. She said, "I can still remember we went to the river and looked for fish or stuff. Yeah. And then we walked up the mountain".

\section{Figure 5:2: Feeding Birds at the Queen's Gardens}

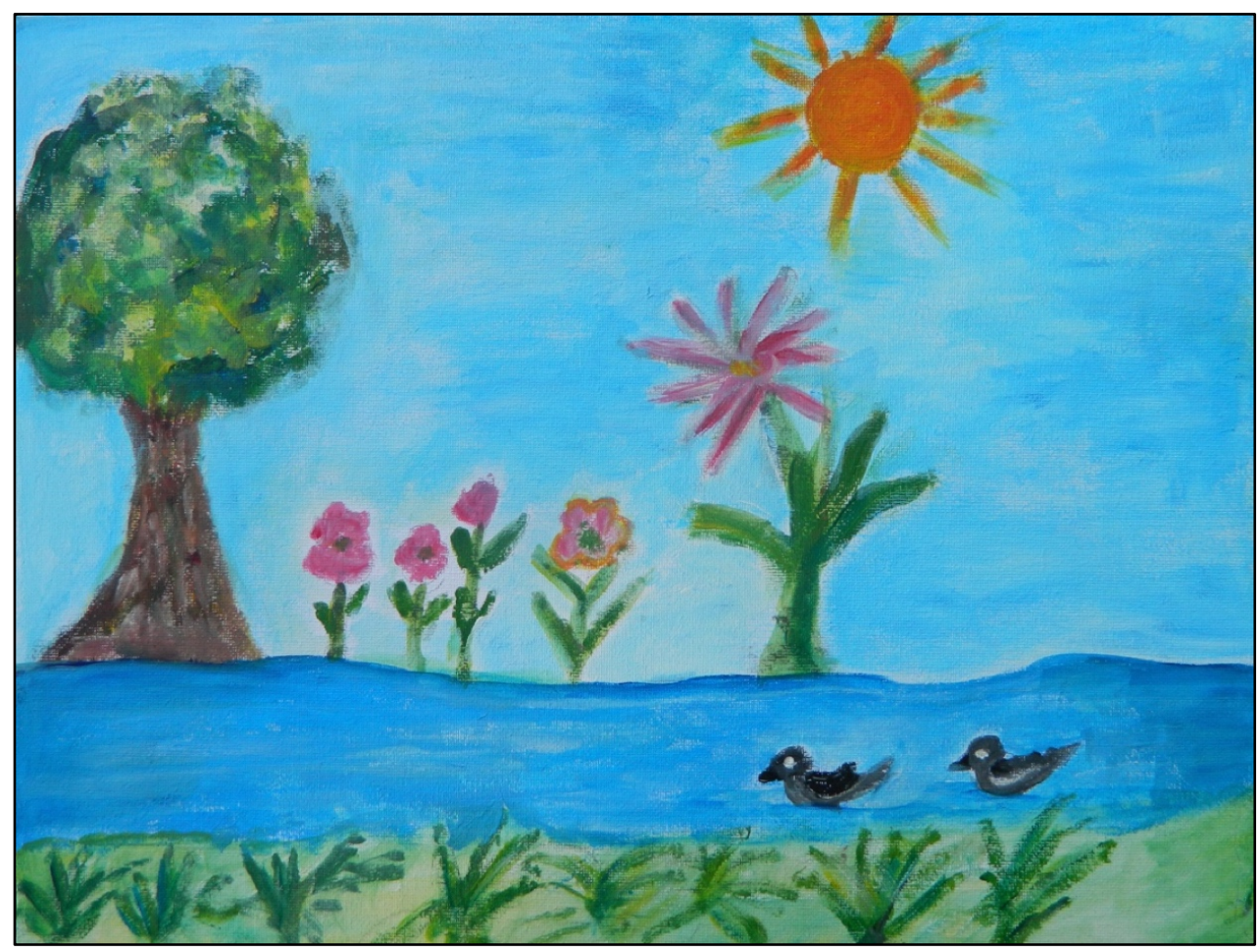

(Source: Painting by Sui Tin Men, 2019) 
Figure 5:3: Walking up the Mountain at Whenua Iti

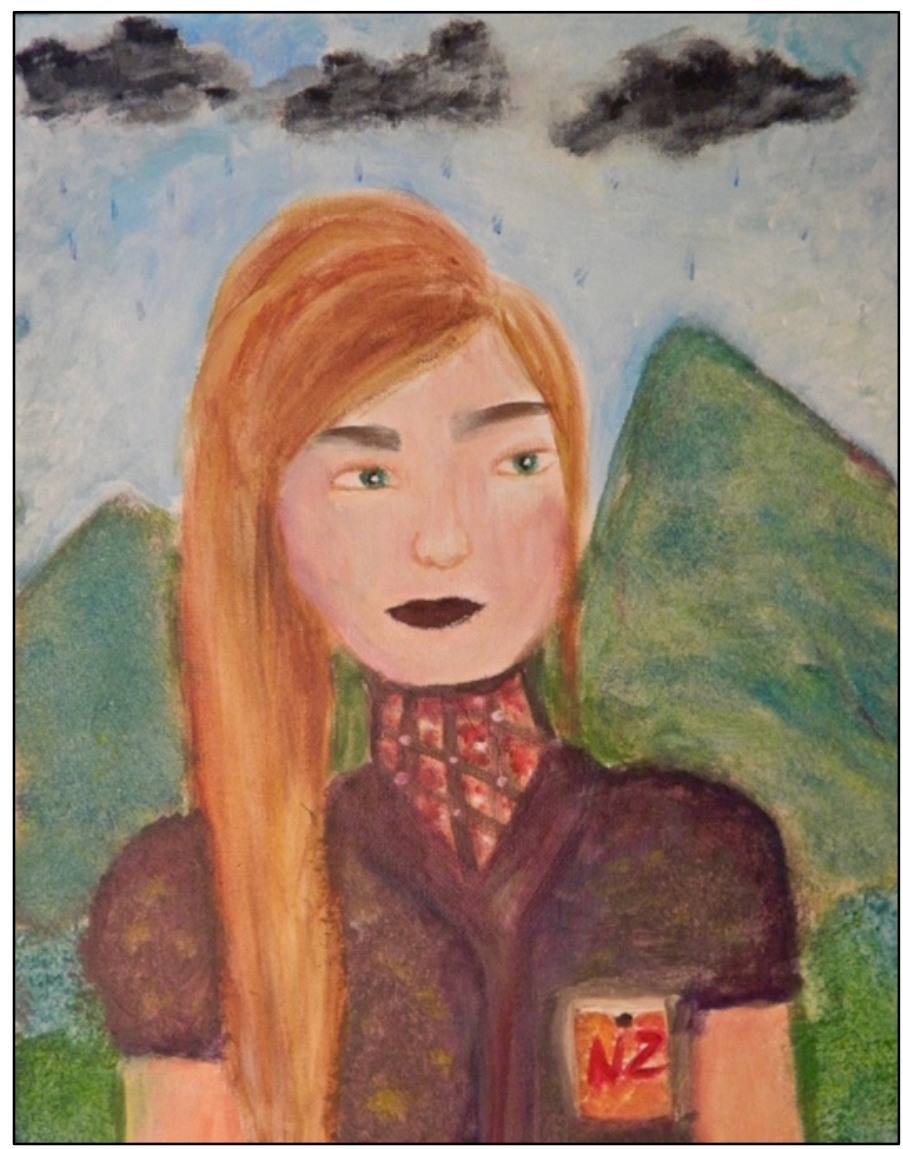

(Source: Painting by Little Ruth, 2019)

Some of the Chin participants had also lived in other countries. To escape persecution and prevalent military abuses during Myanmar's civil war (discussed in more detail in the following section), many Chin people fled across the border to the Mizoram state of India. Some also ventured into Thailand and Malaysia (Human Rights Watch, 2009). Iang Chin Sung said, "Before we moved to New Zealand we lived in Malaysia for about ten years. It is very hot there". Elizabeth also said, "We came from Malaysia, and in Malaysia there are many high buildings". These new place-based experiences were equally significant in shaping participants' conceptualisations of home and expectations for settling in Nelson, and continue to feature throughout this chapter.

The twelfth participant, Mu Pli, identified as 'Kayan'. The Kayan are a sub-group of Red Karen (Karenni people) and are made up of the Kayan Lahwi (Padaung), Kayan Ka Khaung (Gekho), Kayan Lahta, Kayan Ka Ngan, Kayan Gebar, Kayan Kakhi, and Bwe 
(Kayaw) tribes. Kayan make up the dominant ethnic population in Myanmar's small eastern Kayah state (UNHCR, 2014). However, Mu Pli left this state as an infant and spent the following six years in Nai Soi Village in Northern Thailand, before relocating to 'Big Refugee Camp 1' on the Thai / Myanmar border for another six years. Like the Chin participants, Mu Pli noted similarities between the landscape in Thailand and Nelson. She said, "I don't think I see the beach or the ocean [in Thailand], but I do see rivers. Yeah 'cause I lived near rivers and the cliff and the mountains. Surrounded by a little forest behind the house, my home [was] up the mountain".

\section{Seeking familiar places}

Remembering these homely places often evoked warm feelings of nostalgia for the girls and women and enabled them to slip back into a happy time in their life and momentarily let down their guard and relax as they struggled with the confusion of trying to navigate a new city (Akhtar, 1999; Volkan, 1999). Zai Zai, said, “When I arrived at Nelson ... everything is new. So, I'm look around Nelson, I can't remember where I go somewhere! Today I went to somewhere, and tomorrow again, but I can't remember! Everything is new, so, how can I say ... My mind was lost!" To re-orientate herself in space and find somewhere that felt safe and familiar, Zai Zai would visit Broads Field to sit under a particular tree (Figure 5:4). She took me to this location during her interview, and pointing above us she said,

In New Zealand the flowers and tree are not in Chin State. Different kinds, but this tree, we have the same one! Yeah we used to play this game [picks up two parts of an acorn]. This is the plate, and we dig out [the acorn] and this is the cup. Then we put something in here, so this is the plate with the food ... I really miss this tree!

When I asked how she felt being in this place and remembering her childhood, she said, " $I$ feel really peaceful and my mind is really clear. And I can think another kind of new [thoughts]... any problems I can decide it". Thus, being somewhere that felt familiar involved less effort and stress, freeing up space in her mind to think more clearly and feel more in-control of her life. It may also have given her the opportunity to reflect upon other aspects of the past that were weighing upon her mind, and to process any feelings of trauma, loss, or grief (Coughlan \& Hermes, 2016; Room to Heal, 2018). 
Figure 5:4: Playing with the Acorns at Broads Field

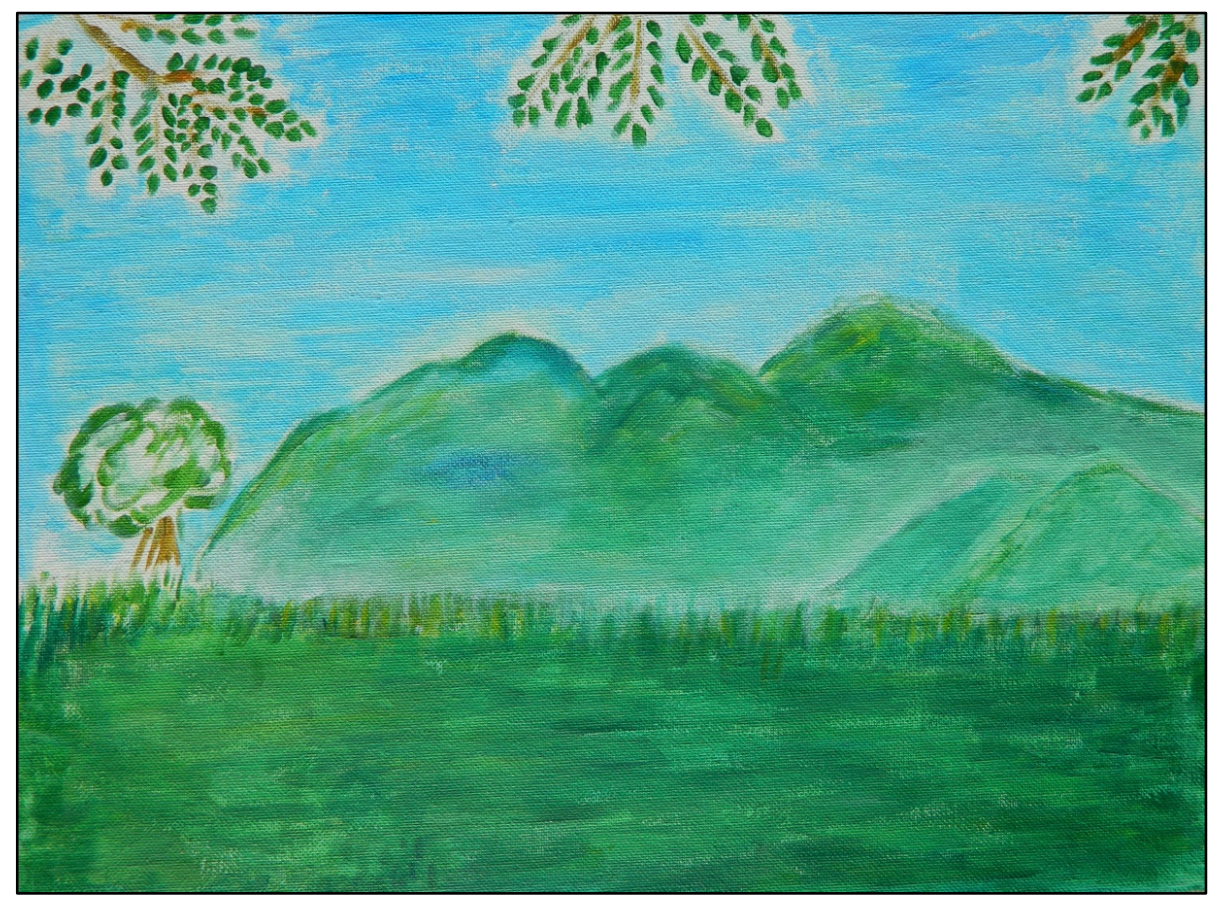

(Source: Painting by Zai Zai, 2019)

Being somewhere familiar also helped Bhutanese former refugees in Nelson to feel relaxed. One of the refugee resettlement practitioners whom I interviewed said,

We used to run a programme where we took the seniors out on monthly road trips ... We went out to Rabbit Island and they sat in the sand and told stories about their home ... about the [refugee] camps. And bringing them together and sitting by the ocean, there was just a sense of being relaxed, and freedom. They talked and reminisced and told stories. Another time when we took them up the hills, they were really reminiscing about growing up in Bhutan.

In this manner, memory can be understood as both temporal and spatial; being inspired by material interactions between bodies and objects, comprised of memorable past narratives, and having a transformative effect upon an individuals' present emotional state and outlook on life. As Blunt (2003, p. 717) claimed, nostalgia not only encompasses a passive act of remembering, but is also 'productive' and "orientated towards the present and future as well as towards the past". 
However, nostalgic memories could also be regressive or pathological and lead to feelings of homesickness, and sometimes a desire to physically return to the lost homeland. When I asked Mu Pli if there was anything that she missed from Thailand, she said, "I'll say the family members and the friends I made. They are going different pathways. That is hard"'. Iang Chin Sung said,

The most difficult thing about leaving Myanmar is that I miss my family. Some of them still live in Chin State. I call them on the phone sometimes to talk. I also miss the food. We can cook Chin food here but there are some things that we can't buy, so I still miss the food.

As mentioned in Chapter Two, feelings of longing for a distant home are well theorised in refugee resettlement research, with particular emphasis on the loss of people, houses, neighbourhoods and culture (Blunt \& Dowling, 2006; Fitzpatrick, 2002; Kale et al., 2019; Kibreab, 2003). However, participants also missed the local terrain and flora and fauna, and rather than focusing on nostalgic memories of the house-as-home, they primarily reflected upon the landscapes of their local villages and regional states. Mu Pli claimed that, in addition to missing her family and friends, she missed,

Not a home [house], but more the village I lived in with the people I hang out with. Those were the times I enjoyed the most and remember most ... [and] the forest. Because in the forest you can find food like bananas or like some other fruits like star fruits and things like that. I love going into the forest with my brother especially and then just take the food home and everyone enjoys the food. Yeah, forest was one of the most favourite places that I love to go.

Meanwhile, Mai Aye Aye said that she missed her family farm, even though it smelt like 'cow poo'. She also missed foraging for food and claimed that, "I want to eat eggplant, in Myanmar it is very long one, here [in Nelson] is very short. I'm looking ... after that, I want to eat banana, in Myanmar there is banana tree, here I am looking, looking, I miss the banana tree". Zai Zai said, "When I was young we go to a forest and climbing a tree and picking a plum and other kinds of fruit. I miss it". Elizabeth added, "You know, in New Zealand we stay home [in a house] a lot. In the village [in Chin State] we go out more and collecting food or collect the firewood for family. We work more in the forest". 
In their research of rural out-migrants who had left Vermont in the $19^{\text {th }}$ and $21^{\text {st }}$ centuries, geographer Morse and historian Mudgett (2017, p. 96) claimed that these geographic relationships remain under-explored. They argued that, "Migrants moving away from rural areas may have deep attachment to non-human aspects of places, in addition to the social worlds they leave behind, a point overlooked in much of the migration research". Whilst these authors have welcomed the emerging body of literature on recreating gardens and green spaces in critical geographies of home (Collins \& Kearns, 2007; Conradson, 2005; Coughlan \& Hermes, 2016; Milligan et al., 2004; Sampson \& Gifford, 2010), they also stressed the need for further research on attachments to rural landscapes, mountains, rivers, and endemic flora and fauna.

Emotional geographic connections had also been overlooked in Nelson resettlement praxis. Whilst several practitioners claimed that former refugees had mentioned missing a specific climate or terrain, others said that they hadn't considered the connection to place, and made comments to the effect that they assumed this wasn't as important as the relationship to people and culture. One practitioner said, "I've not heard anyone talk about missing place. Missing their environment. It might be that I just haven't heard”. Another added that, "It's more about people than place. Family disconnections. And food! Sometimes food is something that comes up. Not so much a physical landscape”.

Not all former refugees will have a strong emotional attachment to the outdoors or consider rural environments to feel homely. There may also be differences with regards to class and gender and who has access to different outdoor places in some cultures. However, for those who do feel connected to nature, their memories of a unique landscape or terrain can significantly impact the way that they perceive new places. One of the practitioners in Nelson said,

I've spoken to people who are rural Burmese, a lot of rural Burmese who miss the rural lifestyle. To a point where I can remember a conversation I had with a guy, who's probably in his forties, who said that if he didn't have children here he would do anything he could to go back to Burma to be back in that rural life that he grew up in and still dreams of ... The pace-of-life. You know, we are so time-focused [in New Zealand], and this is what we find we struggle with [for] many of our clients, especially new clients, is that they are not time-focused and driven. And we come from 
a society where it is all about timing. And that's a real clash ... I've spoken to people who really do miss the physical geography of what they still call home.

This longing to be elsewhere may prevent former refugees from participating in their new communities and investing time and energy into developing a bond with new places (Rosbrook \& Schweitzer, 2010). Thus, whilst Rishbeth and Powell (2013, p. 175) claimed that remembering landscapes can be "a restorative means of envisaging how everyday life continues beyond the strangeness", nostalgic memories can also "sometimes exacerbate the negative shock of the new" and hinder processes of resettlement and place-attachment.

\section{Feeling out-of-sync}

As indicated in the practitioner's comment above, former refugees may also miss a certain 'lifestyle' and 'pace-of-life', or familiar habitual way of living. Psychologist Lewicka (2011, p. 226) claimed that, "When forced to move, people lose not only their social contacts or the familiar view from the window, but they must rearrange their entire set of daily routines and adaptations, and shift to entirely new habits". One of the local resettlement practitioners in Nelson supported this claim, saying that, "The initial shock or trauma of arriving here [in Nelson] is the main thing. It's the first big thing in settling in. You know, getting used to the rhythms and routines of a new place, country, and lifestyle”. These rhythms and routines have also been neglected in resettlement research, although they have been explored in other studies of everyday life in rural and urban spaces (Edensor, 2010; Frydrychova et al., 2017; Lefebvre, 2004; Levin \& Norenzayan, 1999; Lowin et al., 1971; Potts, 2015; Seamon, 1980).

A foundational theory concerning place rhythms in geography is Seamon's (1980, p. 159) concept of 'place-ballet'. Seamon claimed that, over time and in relation to their surroundings, people develop unique bodily movements and habits (which he described as body-ballets), as well as predictable daily patterns (or time-space routines). These subconscious ingrained behaviours occur alongside, and at times intersect with, the habitual movements and daily routines of other people, developing a naturally choreographed 'place-ballet'. He said that the regularity of these interactions, "May also produce a climate of familiarity which participants appreciate and to which they grow attached". Other influential work in this area are phenomenologist Merleau-Ponty's (1962) 
ideas of habit, and a recent collection of studies in geographer Edensor's (2010) book, 'Geographies of Rhythm: Nature, Place, Mobilities and Bodies'.

The rhythms and ballets of the city are often perceived to flow faster than those in rural places (Frydrychova et al., 2017; Lowin et al., 1971), and can create a sense of desynchrony for those migrating between rural and urban environments. Some cultures are also understood to be more fast-paced or time-focused than others (Levin \& Norenzayan, 1999), and a faster pace of life may be associated with a heightened sense of vibrancy or vitality, which can be viewed as positive or negative depending on what people are used to and their personal preferences (Kale et al., 2019; Kamri-McGurk, 2012).

For example, Elizabeth claimed that she immediately felt disappointed by the lack of movement and excitement when she first arrived in Nelson. After leaving her small village in Chin State she had spent a year in Kuala Lumper (a city in Malaysia with a population of approximately 1.8 million), and had become enthralled with the excitement of living in a thriving urban metropolis. She said,

Kuala Lumper is a very busy place, [with] so many people. Like restaurants, cafes everywhere and um, you know, like karaoke and many shopping malls. So, um, when I arrive in New Zealand I thought New Zealand was going to be like that, like Kuala Lumper. But when I arrived [in Nelson] it's very different. And I think, oh, it's like my village [in Myanmar]! No tall buildings. I thought it was going to be a big city. First thought, Oh! It's going to be boring!

This perception of small cities or rural areas as boring is common amongst former refugees resettling in New Zealand, a country with a land mass of $268,021 \mathrm{~km}^{2}$ and population of five million (Stats NZ, 2019b). One of the resettlement practitioners said, "I've heard it from other migrants who come from big cities, and in Nelson it's like wind whistling down the streets. Like, where is everybody?" Another practitioner, who had recently worked with members of the Colombian community, shared a similar experience, saying,

[Colombians] can be very colourful with their clothing and their Sambas and bands and the football team, and it's all a bit dull here and that can be a bit of a pull down. And I think, you know, compared to perhaps the colour and the vibrancy that they are 
used to in their cities and on their billboards, it's a chaos of colours in many places so I guess that's a big stimulation difference.

Burmese former refugee participants in Kamri-McGurk's (2012) research in Porirua, Wellington also talked about how New Zealand felt too quiet, boring, and lonely after they had spent time living in Malaysia, and a resettlement practitioner in my Masters' research (Kale et al., 2019) claimed that many former refugees preferred to live in Auckland (New Zealand's largest and most multicultural city) to retain the sense of vitality to which they are accustomed.

However, Zai Zai disagreed that Nelson was dull and boring. In contrast, she found it to be too big, busy, and crowded. She said, "The town, I'm not really comfortable, not comfortable ... [it's] busy. Like um, this [sitting in the park] is relaxing. So, town is too many people. I don't want to go in crowded places". Iang Chin Sung said, "I don't like to go to the nightclubs. Some people drink and smoke and there is dancing, it is very loud". She added that,

I am worried when I am driving. Sometimes the other drivers are dangerous, and the roads are busy and loud. In Myanmar I didn't drive. Most people in Myanmar are walking, biking, some have motorbikes and sometimes there are cars, [but] the roads are not busy.

Ruth was also overwhelmed by the traffic and claimed that, "I don't like Waimea Road because too many car, busy road”. Navigating dynamic new rhythms and 'busy' places can be stressful. It can also demand high levels of attention and concentration, which could diminish a person's energy levels and leave them feeling exhausted or fatigued (Hammitt, 1982; Kaplan, 1978). These feelings could potentially discourage individuals from accessing certain parts of the city and engaging in public life.

The girls and women also felt out-of-sync with Nelson's seasonal rhythms and cycles (Lefebvre, 2004; Potts, 2015). Ngun said, "In Myanmar we have three seasons. Rainy season, summer, and winter. In winter very cold like New Zealand. Rainy season always raining, no cold, just raining. Summer is hot”. Over time, the girls and women had harmonised their bodily rhythms, social practices, and routines with these seasons (Seamon, 1980) and instinctively come to know what to expect and what was safe. Sui Tin 
Men said, "Sometimes [we grow] corn, sometimes rice. Sometimes uh June, July, August, you know fruit or vegetable a lot in the farm, and eat corn and pumpkin, cucumber, bean. A lot a lot. But, uh, summer and no more. Gone”. Meanwhile Iang Chin Sung said,

Two places that I felt attached to in Myanmar are my house and the river. It would take us two hours to walk from my house to the river, then two hours back home. We would do this a lot in the summer, but in the rainy season we can't go because the river is too full and it is dangerous.

When the women moved to New Zealand, these reliable synergies and expectations were disrupted. A couple of participants expressed confusion over why their plants in Nelson weren't growing as they expected, showing me the leaves and suggesting that perhaps it was due to the drought that we were experiencing. Jenavi also talked about how the months of the year were now out of sync with the temperatures she expected to feel. She said that in Chin State, "November, December is cold. Different to New Zealand, here is hot".

These geographic differences also affected the rhythms and routines of local flora and fauna, and each participant's spatiotemporal relationship with these non-human entities. When we were out mapping the multisensory experiences in her garden, Jenavi mentioned that her mother never wore a watch in Myanmar because she knew it was lunchtime when the cicadas started to sing. She had become accustomed to these audible rhythms of when to work and when to rest in the rice fields, and thus, when the cicadas in Nelson started to

sing before noon, her perception of time was disrupted. These bittersweet memories and moments of discord reminded the girls and women how geographically and culturally removed they were from their former homes, and during their interviews they often trailed off their stories and gazed into the distance, or mentioned that someday in the future, it would be nice to return and visit Myanmar.

\section{5:2 Navigating fearful landscapes}

\section{“I don't want that to happen again"}

Whilst the girls and women spoke very fondly of their homes, nostalgic memories could also be romanticised. Drawing on the work of Anthropologist Tonkin (1992), landscape 
researchers Rishbeth and Powell (2013, p. 172) argued that, through new experiences, "Memories of place can be heightened or assigned new relevance, reshaping a narrative that can promote idealised memories of past experiences and places". As our interviews progressed, participants' nostalgic memories of the past became interspersed with more fearful memories of a violent and fractured sociopolitical and geographic landscape, and villages, mountains, and forests that were no longer safe nor even habitable (Blunt \& Dowling, 2006; Hinton et al., 2004; Kale et al., 2019).

In my interview with Ruth and Little Ruth, they quietly discussed how loud noises triggered memories of people "shooting each other" in their village in Chin State. I could see that Little Ruth felt uncomfortable with this memory as she subconsciously dropped her gaze and began to shuffle around in her seat. Specific sounds also evoked troubling memories for Elizabeth. As I was about to conclude her interview, she suddenly exclaimed, "I have one, uh, sad memory". She then angrily described the terror that came with hearing a knock at the door late at night and associated this sound with the arrival of the Bamar army, who would come to her village in Chin State to conduct raids,

As you already know, the military uh, rules Myanmar. And oh, it is horrible, horrible. I don't want to [be] there. You know, every night in the village they go house to house to see any visitors. At night, you all sleep peacefully and then, ВООМ! ВООМ! BOOM! Knock at the door. That makes us worried a lot. Sometime what they see in our house they just took it. I hate it so much! They um, you know, they just took somebody's stuff like their belonging ... In the other village sometime, the soldiers fight the village leader ... they fight them, they kick them, they bleed. If the military came to our village everybody is scared. They do whatever they ask. If they, like for example, 'bring me ten chicken for military to eat', and you find only five chicken, they fight them. They kick them. They use with the gun [Shows hitting motion] ... 'You don't do what I say', you know? No human rights. No rights. We must do what they say. They act like they are the king.

She added, "I don't like it, I don't want that to happen again, that kind of memory".

In 1824 Myanmar, then known as Burma, was colonised by the United Kingdom of Great Britain (UK). Ethnic tensions soon formed over rising anti-colonial sentiments and contradictory ideologies of governance. When Burma gained independence from the 
British in 1948, and the dominant Bamar ethnic group came into power, supporters of communism and many ethnic minorities believed they were being treated unfairly and excluded from gaining political power (Oxford Burma Alliance, 2018). This discontent led to ongoing insurgencies, which the Bamar government responded to with force.

In a desperate bid to escape the violence, many ethnic minority families fled into the rainforests, where they struggled to find food, grappled with malaria, and had no access to medical care (Smith, 1994). The Bamar army forced others into government designated relocation zones, where they had little opportunity to make a living and lacked the social networks that provided safety and emotional support (Smith, 1994). From these relocation zones (and in many minority states) there have been horrific reports of sexual violence perpetrated against women and girls by government troops (Shan Women's Action Network \& Shan Human Rights Foundation, 2002). Chin residents have also reported increasing systematic sexual violence against men (Physicians for Human Rights, 2011), and the Bamar government has further been accused of recruiting young boys as disposable soldiers (Smith, 1994).

On November $7^{\text {th }}, 2010$ Myanmar held its first elections in two decades and transitioned to a civilian-led government (Oxford Burma Alliance, 2018). In 2011 the new government began a process of political, economic, and administrative reform. These reforms included the establishment of the National Human Rights Commission, general amnesties of more than 200 political prisoners, institution of new labour laws, and relaxed media censorship. Whilst these changes were initially viewed as progress towards a peaceful future, the elections were claimed to be unfair as the same Bamar military generals continued to rule as civilians, and ethnic persecution and violence continued (Oxford Burma Alliance, 2018). Zai Zai said, "The [Bamar] government is not really good. Now Myanmar is democratic nation, but only the name. How we are living in Myanmar, there is a lot of suffering, people killed each other and ... uh, how can I say, children, kids, sex assault and killed. Too many, too many”.

The year that these purported 'reforms' took place, Freedom House (2011) ranked Myanmar as the third most repressive country in the world after North Korea and Somalia. In a damning report the following year, Human Rights Watch (2012, p. 300) claimed that, 
"The Burmese military continues to be responsible for abuses against civilians in conflict areas, including forced labor, extrajudicial killings, sexual violence, the use of "human shields," and indiscriminate attacks on civilians". By the end of 2016 the UN had documented 490,289 refugees from Myanmar, making it the eighth largest refugeeproducing nation in the world (UNHCR, 2016).

Whilst the girls and women had physically escaped the war, they retained the sense of fear that such harrowing experiences had evoked. Drawing on geographer Hadi Curti (2008) and anthropologist Kidron's (2012), memory research, geographers Drozdzewski, De Nardi, and Waterton (2016, p. 451) claimed that, "The experiencing body becomes the site of conflict and postmemory, where memory and reverberations of a violent past and loss are embedded in somatic modes of being". Or, as an individual in the New Zealand Red Cross Youth Resettlement Review explained, "You have seen something happen but now you have come to a different country it is always going to impact you. Your past is always your past and will always be there" (O’Connor, 2014, p. 30).

This spatialising of fearful memories within the body could be problematic, as geographer Pain (2001, p. 911) argued that fear has "tangible and serious effects on social interaction, use of space and quality of life". As mentioned above, several of the participants tried to avoid places where there were loud noises (such as the city, nightclubs, and roads). This was partly due to the desire to recreate a familiar homely feeling of a quiet rural place, though it may also serve to avoid the loud unexpected noises imbued with memories of warfare (Hinton et al., 2004).

Visceral stimuli such as the clenching and rumbling of an empty stomach could also evoke fearful memories of poverty and hunger. Elizabeth said,

In Chin State, food is very difficult. Most of it we just collect from the forest and many hills, its difficult just to plant plantation. So, food is very difficult, we collect in the forest one day you know, not enough for a week or something like that. So, that's ... the place [used to be] very peaceful but the food very difficult to live. But in Myanmar, like down, down, down, like Mandalay, more food to eat, more vegetable. They have, [that part of] Myanmar is very flat so they can do planting whatever they want ... But not in Chin cause hill, too many hills. 
With the confiscation of ethnic-minority group's land by the Bamar Army, and the additional challenge of trying to grow and collect food during times of warfare (Smith, 1994), residents often went hungry. Little Ruth added that when living in Chin State, “We have to work hard for our stomachs".

The girls and women also spoke about the sound and movement of the wind in Nelson evoking 'scary' memories of natural disasters. Sui Sung said,

[In Myanmar] my husband sometimes three or four months he is gone to work so [I] stay in mum's house. Because I am scared for night-time. I am very scared! Why? Sometimes it's very windy WOOO00!!! Very scary! You know W00000!!! Too windy. I'm very scared. Sometimes you know it's raining too much. Sometimes lightening coming ... АНHНH!!! Very scared!!! ... Sometimes near my house, before I'm not too much scared, but um it start for I'm scared when near my house big tree is too much windy is blew over and house is broken. One [neighbor] ... he is not died but ... [indicates that he's injured his leg] ... It's very scary. Is before I'm young, I think ten year old this one. My neighbour, yeah, I think ooooh, very scary wind.

Cyclones, floods, and landslides are common in Myanmar, and past largescale events of this nature have been particularly devastating (International Federation of Red Cross and Red Crescent Societies, 2008; Thomas, 2016). For participants from small rural communities, these natural disasters could be equally as frightening as warfare.

Mu Pli claimed that,

[In Thailand] our roof is made of leaves that we made our own ... so it could blow off. Or, like, it was raining too heavy and could create a hole in our roof and it would leak and that gives us problems because that means we have to rebuild that. For the leaves then we have to go out into the forest and collect leaves and also bamboo, collect that from the forest. Everything from the scratch basically and we're making them our own ... But it was pretty scary sometimes when there is bad weather coming. We will go to the home, our friend's home or neighbours home that is the most stable. We will go and stay underneath their house, just so everyone is safe from the bad weathers. That was a scary time ... If the wind blows really hard here [in Nelson] I do get scared. Other than that, I feel very safer here. 
Fire had a similar impact. In February, part-way through the multisensory mapping project, a huge forest fire broke out in the neighbouring district of Wakefield, lighting up the sky at night and filling Nelson City with thick dry smoke. The girls and women acknowledged that the fire prompted no immediate threat to their houses and families in the suburbs of Toi Toi and Stoke; however, the view of the burning hills and the visceral inhalation of smoke evoked troubling memories for individuals who had previously experienced devastating fires or other smoke-related incidents. Mu Pli said,

I used to see this person back in Thailand with a ... I think he smoked all the time, but he got like a hole in his throat ... so, it really scared me. Whenever I smell smoke, my memory that will just drop back and I'm just like no ... that's part of it. And another thing is like when I breathe it in I don't feel good. Like, it makes me cough, it feels unhealthy, bad for my health.

On her street map she extended this dislike for smoky places beyond the smell of cigarettes to include any kind of smoke or gas that might evoke an unpleasant unhealthy smell and visceral coughing sensation. Sui Tin Men also claimed that, "Sometime one family and one people [in Myanmar] went to a fire in farm. And big tree a little bit dry, dry, dry. One weeks, two weeks dry, and fire start. And my family and one [other] family, one people go [to] the fire. And very hard. Women, and, too hard. I don't like". As with the lack of research on nostalgic memories of rural landscapes and non-human entities, traumatic memories of natural disasters are under-explored in resettlement research. However, these experiences can also take an emotional toll on former refugees and impact everyday resettlement and wellbeing.

\section{Hope and empathy}

Whilst fearful memories could be uncomfortable, they also helped participants to feel relatively safe in Nelson and hopeful for a less violent and more prosperous future. Several of the girls and women claimed that the silence at night and the absence of sporadic gunfire and armed military men walking the streets was comforting as it offered a sense of peace that had eluded them during times of conflict. When I asked Elizabeth if she felt safe in Nelson she exclaimed, "Oh yes! Yeah. A big difference. You know, if that kind of thing [violence] is not happening, of course, everyone like to stay [in] their own home. [But] as 
a mum especially, I feel [that Nelson is] safe for my children. Most important is my children”. One of the practitioners said, "We did research about aging in Nelson, and [the former refugee participants] said they feel safe here and they don't want to go back. They want to see their family, but they don't want to go back and live in that unsafe scary place”.

Participants also said that they felt thankful for the abundance of food, stable shelter, and opportunities for education and employment to which they now had access. Mu Pli said,

I guess that food here in New Zealand is better ... in terms of we can get more food. Here it is like 4 or 5 eggs we can eat a day, or just for breakfast! But back in Thailand, even if we have just one egg we will have to share with the whole family. In that sense I can say that everything is so much better in New Zealand ... [And] I feel safer here [in my Nelson house] because it's more covered and bigger and it looks more stable. It feels more stable. I think, what would it be like if I hadn't moved down to New Zealand? I guess keep studying. I have no clue what job I would have got, other than helping out in the house. The future is very blurry when I think about if I'm still there [in Thailand]. It's very hard to get a job back there so I don't see any ways of getting to the future ... In [New Zealand] I see a lot of future for me. I just see a lot of paths that I can take. I see choices that I can make. In terms of school there are many careers I can study for. And I can have any goals that I want, there are many things I could try ... I'm just happy with my life now. But there are areas to improve on, personal growth, things I want to achieve. Hopefully I'll be able to do them.

Hope is defined by health economists Pleeging et al., (2019, p. 2) as, "A strong desire that is actively pursued, while it is uncertain that this desire will be fulfilled". Researchers have claimed that high levels of hope can enhance an individual's emotional ability to cope with stressful situations (Ciarrochi et al., 2007; Fredrickson, 2001; Valle et al., 2006), and take an active role in cognitively setting and achieving long-term goals (Snyder et al., 2002). Thus, psychologist Jevne and self-help author Miller (1999) claimed that hope is an essential aspect of living a quality and meaningful life, particularly for refugees who face a myriad of resettlement challenges. 
Geographers Pain and Smith (2008, p. 7) claimed that, "Fear and hope are two sides of a single coin; they cannot be uncoupled but one is often more visible than the other". Similarly, geographer Wright (2008, p. 223) said,

Resisting fear cannot be associated only with meeting fear head-on. It also needs to be recast as the generation of hope, of creating empowered subjects and of generating alternative realities that make fear, if not redundant, no longer central to the way that people live their lives.

Whilst decentralising fear and focusing on hope is pivotal in finding ways of 'getting to the future', in practice this could be extremely difficult for the girls and women as their memories of a violent and fearful place were not only remnants of a troubling past, but harsh reminders of the threatening reality still faced by many of their loved ones. Elizabeth said, "Now [the conflict] is not happening. I mean still happening some place though. Yesterday I read the news and some place happening. You know, we can't say it's safe. Still one month later, or three month later, it's still happening”. Ruth claimed that her brother-in-law (Little Ruth's father) had recently broken his leg in Myanmar, and said,

This why I worry ... And the food, no. Money, my husband give me the money take care ... send money back. Buy food my country. And my niece daddy he can't work. No working only stay at home ... My family is happy but I worry this (gesturing towards her niece) daddy and ma.

The precarious conditions in which some of the participants' relatives lived was evident during my 2019 visit to Mae La refugee camp on the Thai / Myanmar border. Through an interpreter, a resident from the camp claimed that they were struggling with a decrease in food rations, the inability to leave the camp and make a living, substance abuse, ongoing health problems, and mounting pressure from the Thai Government for asylum seekers to return home. Meanwhile, those who had remained in or returned to Myanmar found their land confiscated and their villages destroyed. They also had to cope without electricity, infrastructure, or jobs, and endure ongoing persecution and the threat of being maimed or killed by landmines (Field notes, 19/09/2019). Whilst participants were hopeful for their own future in New Zealand, and hopeful that their distant loved ones may be able to one day share that future with them, it was easy for this fear-for-others to remain a central and dominant aggressor in their lives. 
Feminist scholar Ahmed (2008, p. 10) claimed that, "Emotions are quite sociable. We are moved after all by the proximity of others. We feel with and for others". Such sociability and concern for others was also evident in Zai Zai's comments about the Wakefield fire. She said, "That day [when the Wakefield fire started] I'm really worried about people. And oh! Our Nelson! How?! I'm really sad from Wakefield people, I'm really sad”. Whilst participants were drawing upon personal memories of how it felt to be in a dry hot fire, and what it meant to be forced from one's home, this recognition of and feeling with and for others prompted members of the Chin community to donate a substantial sum of money towards assisting local Wakefield residents who had been displaced. In this manner, participants and the wider refugee-background community were building on relatable emotions of fear, loss, and hope to connect to their new city and fellow residents (Waite et al., 2014). As devastating as these events can be, the tendency to come together and take responsibility for 'our Nelson' may be an important step forward in emotionally unifying diverse ethnic groups and paving the way for collaboration to build a safe, hopeful, resilient community.

\section{5:3 Maintaining a sense of homeliness}

\section{Mediating between the past, present, and future}

These ambivalent feelings between the past, present, and future highlight the importance of setting aside time and space for former refugees to mediate between different experiences. As mentioned in Chapter One, current refugee mental healthcare in New Zealand has focused on such mediation through Western practices of counselling and talking through painful memories (New Zealand Red Cross, 2018; RASNZ, 2018). Whilst talking about the past may relieve tension and generate hope, remembering is not always easy and during the interviews the girls and women did not always feel comfortable sharing traumatic or sensitive stories. This could be compounded by having English as a second language.

When I asked Ngai Ciang Sung and Mai Aye Aye if they considered themselves to be refugees now that they lived in New Zealand, Ngai Ciang Sung paused for a long time and then said, "We don't really want to talk about that". Meanwhile, Mu Pli said, "I don't think about it [Thailand] a lot of the time. But if something reminds me of it I'll get into it 
and reflect on how it used to be back there ... But mostly I try to remember only good memories". Suppressing or forgetting the past may also be beneficial in enabling some individuals to move on with their lives and beyond traumatic events. However, if overwhelming sensory stimuli and negative memories of home or feelings of loss are not addressed during the initial stages of resettlement, they can also build up over time and have a negative long-term impact upon wellbeing (Hinton et al., 2004).

Talking also does little to compensate for the desired people, places, rhythms, and lifestyles that have been lost through forced displacement and resettlement. Because memory is embodied and relationally constituted through interactions with the material world (Blunt, 2003; Drozdzewski et al., 2016), a more tangible process of healing and recovery may be needed which centralises everyday multisensory and emotional experience. There is also a need to shift beyond the focus on trauma to recognise that positive nostalgic memories have an important role to play in healing and feeling well.

One practical way in which participants mediated between the past and present was through video-calling family via Skype, Facebook and WhatsApp. Ngun said, "We have one [son] in Myanmar, so we show him our house, our flat, we record like this [with the webcam], so we show him”. Mu Pli also said that she would video-call family in other places. 'We show each other, 'it's sunny here today, look, look!' That's what I do, 'Have a look it's so beautiful!' And we do compare the place that they live in and here. 'What's it like there? This is how it's like over here'. That's how we just keep talking'. Zai Zai said that she even live-streamed her wedding to include those who could not attend in person. She said, "When we have our wedding ceremony and they want to see, can show them the place we live. This is our city Nelson. And, 'Oh! It's like our city in Chin [State]','.

These multidimensional global communication networks helped participants to see and hear distant people and places and maintain some of the intimacy that long-distance relationships might otherwise come to lack (Elliot \& Urry, 2010; Marlowe, 2019; McNeill, 1994; Ozkul, 2013). One practitioner claimed that, "Things like Skype and Facebook, these are critical ... It's essential. That communication, however it happens, is vital to their wellbeing. And it is a really important visual and auditory requirement for wellbeing I'd suggest in most cases”. In refugee resettlement researcher Marlowe's (2019, p. 179) study 
of social media and forced migration in New Zealand he also said, "All participants noted how being connected to those they are separated from by geographical distance was essential to well-being and participation in resettlement contexts".

Being able to share their new experiences with distant others also had a positive influence on participants' relationships with Nelson as they were actively interacting with their new surroundings and familiarising themselves with their new environments through the process of filming. However, they were also distracted from their present location as they maintained a virtual presence in a distant homeland. This distraction could have a similar detrimental effect to losing oneself in nostalgic memories and lead to feelings of homesickness and a desire to be elsewhere. Yet, in Marlowe's (2019, p. 179) aforementioned research he claimed that, "While there are potential concerns that ongoing transnational interactions could be a disincentive to engage with everyday lives and local politics in countries of resettlement, participants were nearly unequivocal of its importance to meaningfully settle in New Zealand”.

\section{Enacting the past in the present}

Another way in which participants were mediating between different times and places was by bringing homely practices imbued with particular senses into new places of settlement (Collins, 2008; Hage, 1997; Kale et al., 2019). This approach centralised hope and the 'productive' potential of nostalgia rather than lamenting the impossibility of returning home (Blunt, 2003). From the moment they arrived in Nelson, the girls and women had recreated positive aspects of their former homes by playing traditional music, speaking Indigenous languages, setting up cultural objects around their houses, and cooking familiar dishes. Ruth wrote on her street-map, "I like cooking in my kitchen. I like cooking the beef and spicy". This sentiment was further reflected in her painting of throwing spices into a pot in her new kitchen (Figure 5:5). Iang Chin Sung also said, "Our food tasted hot. It is different food from New Zealand, but its okay because I can cook the same food here as well in my kitchen". Reflecting on a refugee cooking initiative in the UK, one of the practitioners said that traditional cooking had, "Made a big difference, both in the smells in their kitchen and the taste in the food they were eating, and it just was a real comfort". 
Figure 5:5: Cooking Spicy Food in the Kitchen

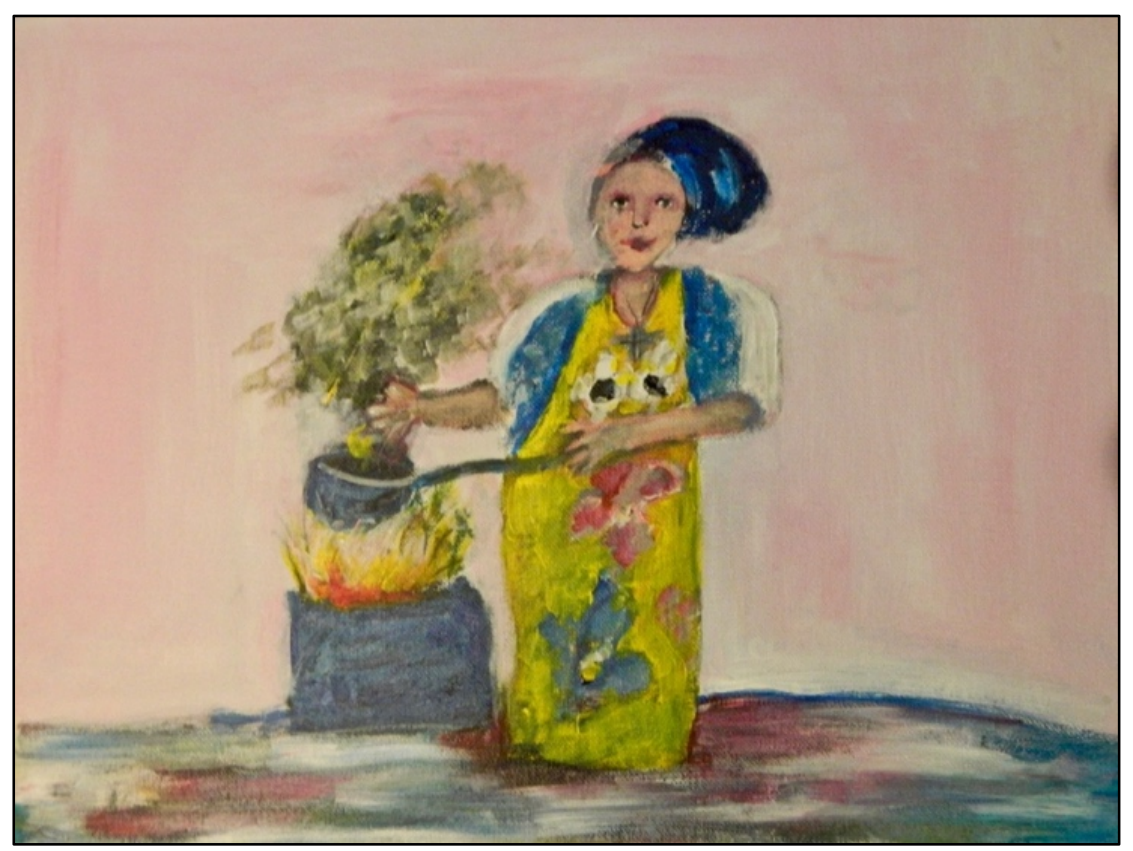

(Source: Painting by Ruth, 2019)

Participants also engaged in familiar outdoor activities such as fishing at the Nelson Vickerman Street Lay-up wharf (Figure 5:6), walking in the hills surrounding the Nelson CBD, taking day trips to the beach at Rabbit Island or the Maitai river, and camping at popular local holiday places like Ruby Bay or Quinney's Bush (See the place-attachment map in Appendix Nine). Zai Zai said, "In the camping we cook food, we have a BBQ, and we swim ... My kids really like outside and they also really like going camping”. They also worked hard to cultivate their own vegetable gardens in Nelson, and Ngun claimed that she didn't use the gardening gloves that her daughter had bought her, because, "In Myanmar we just use our hands!" (Figure 5:7). These experiences were similar to those of other former refugee women from Myanmar, who had continued familiar gardening practices from home as they resettled in Porirua (Kamri-McGurk, 2012).

The desire to recreate gardens and get one's hands into the soil during rural to urban resettlement has also been well documented overseas. Recent studies in this area include sociologists Coughlan and Hermes' (2016) research with Somali-Bantu former refugees in New York in the US; interdisciplinary scholars Abramovic, Turner, and Hope's (2019) research with Burmese former refugees in Canberra, Australia; and sociologist Biglin's 
(2020) research with former refugees from diverse African countries in the UK Northwest. Practice-based initiatives in the UK also include artist Barnes' (2018) Companion Planting project in Bristol, and Room to Heal's (2018) therapeutic gardening project in London. On their website, Room to Heal (2018) claimed that,

For many Room to Heal members, life 'back home' involved working the land, tending to plants and working on farms or smallholdings. Violent rupture from the land and the challenges of life in the urban jungle of London mean that gardening together as a group can take people to a happier time of life, connecting people to their pre-traumatised selves. Relating to life through the metaphor of nature, the changing seasons, the responsibility of tending to something, slowly helps to heal the "shattered self" that had been deeply damaged through torture.

During my 2018 UK research trip, I visited one of the Room to Heal gardens and spoke with an on-site therapist. She stressed that their successful resettlement outcomes were due to the holistic, integrated nature of their services, whereby individuals were not just accessing counselling, but simultaneously addressing aspects of their emotional, mental, and physical health through meaningful gardening, socialising, talking, and sharing food. This integrated approach established a sense of community, and, rather than positioning former refugees as vulnerable traumatised individuals, focused on their role as active community members with the ability to transform their own lives and the lives of others (Field notes, 11/09/2018).

\section{The challenges and benefits of recreating aspects of home}

As could be expected, there were also significant challenges in recreating familiar experiences in a foreign geographic and social landscape. One of these challenges involved the different tools and bodily movements (or body-ballets; Seamon, 1980) involved in fishing. On several occasions during the interviews, the women had jumped up out of their chairs to act out the specific movements involved in fresh-water fishing in Myanmar. They used their whole bodies to demonstrate how they would hold a woven basket in the river and shake a rock above a fish to direct it into the basket. They also demonstrated the contrasting jerking-and-reeling arm and hand motions needed to hook a fish on a rod when fishing in the ocean the 'New Zealand way'. 
Figure 5:6: Fishing by Sealords

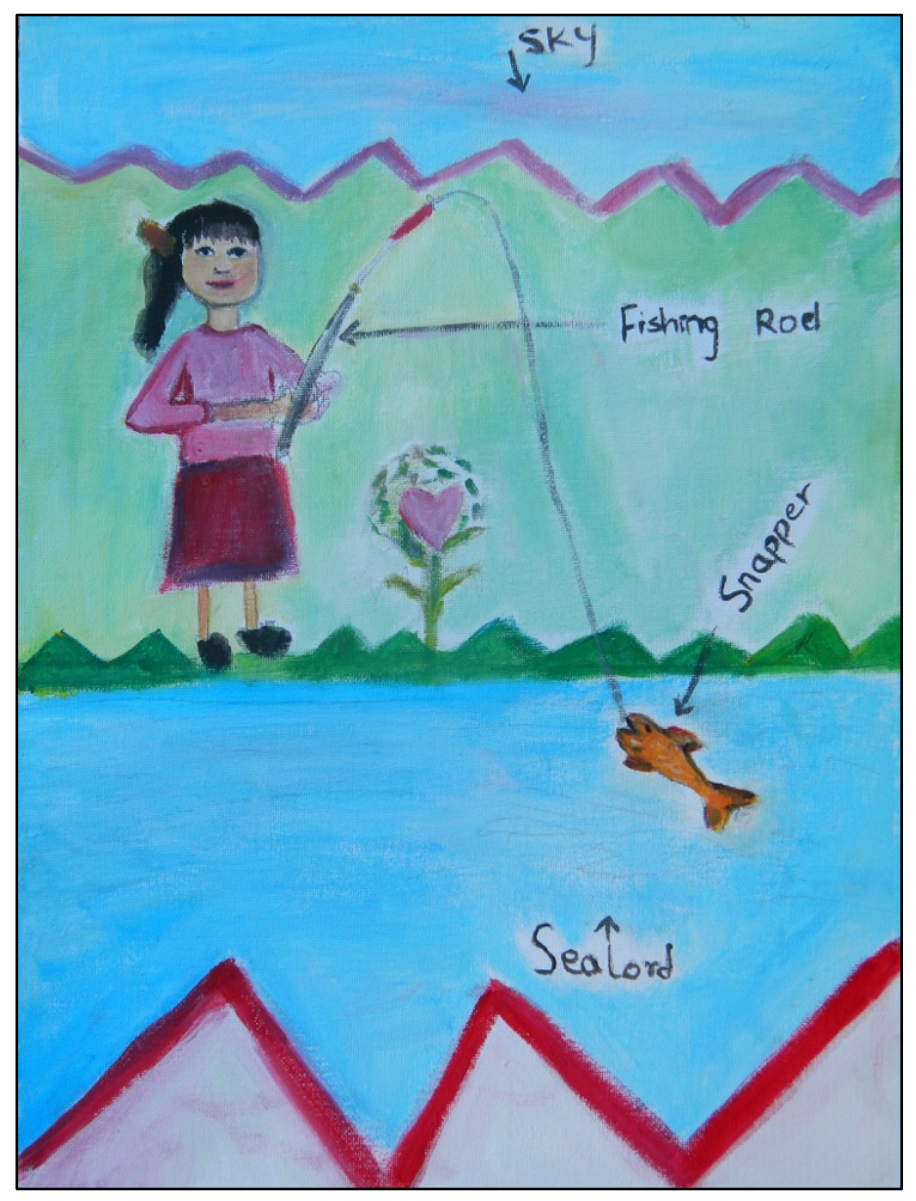

(Source: Painting by Iang Chin Sung, 2019)

Figure 5:7: Gardening without Gloves

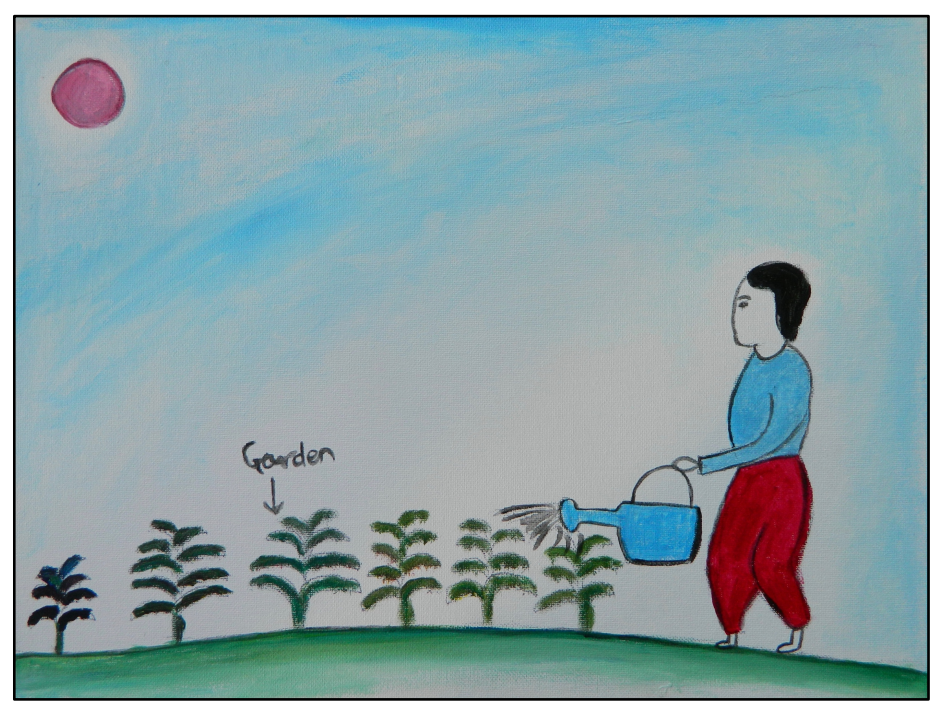

(Source: Painting by Ngun, 2019) 
These enactments drew upon vestibular, proprioceptive, and interoceptive senses (Champagne, 2004), and explored the familiar and unfamiliar ways in which bodies move through space to optimise a specific place-based activity (Seamon, 1980). By standing alongside the women and copying their movements, I realised how daunting a new place could be when ingrained habits, such as the unique flick of a wrist that I had spent years perfecting to cast my own fishing line, suddenly became redundant.

Another significant challenge was that participants struggled to grow familiar produce as seeds endemic to Myanmar were difficult to import due to New Zealand's strict biodiversity regulations. Nelson's temperate oceanic climate and different soils and wildlife also made growing tropical produce problematic, and different flora and fauna and new ways of cooking altered traditional flavours. Sui Tin Men claimed that, "Sometime uh, fire cook taste better". Elizabeth agreed, saying,

Yeah in my country we use the firewood we roast like a meat or corn or potato in the ash. And also like we roasted the birds, the wild birds. And that is more tastier, like natural way ... we have some kind of trap, like um in my country we have flower is called rhododendron. That is our Chin national flower and we have lots in the forest. The birds like to suck the pollen and then uh, we like put a trap in there. Ah, natural, birds so yummy to roast. You can't see in New Zealand!

Elizabeth also demonstrated how different plant life could hinder the re-creation of traditional celebrations like Christmas. She claimed that,

In the village [in Myanmar], Christmas time is the favourite time for every children. In Christmas there is Cherry Blossom everywhere and it makes people happy, Oh, this is Christmas time, you know, of the season ... All those in high school in the city they all come back to the village for Christmas to spend time with the family. Everyone see each other, and we prepare for Christmas and go Christmas carolling house to house with our friends. And, oh! That is the most fun time. Yeah, in our life! ... And especially Christmas time lots of food. Like culture food. Sticky rice, with the banana leaf, that kind of special food ... That is childhood memory. Is the best. Nothing to worry about ... but [in Nelson it's a] different feeling.

She explained that they still had carols and nice food in Nelson; however, without the cherry blossom and banana leaves that characterised a unique geographic and seasonal 
experience, Christmas didn't feel like Christmas. Zai Zai said, "We celebrate not really like Myanmar because the place are different and our environment are different people".

The girls and women acknowledged that there would always be aspects of home that they would miss, and which could not be re-created in New Zealand. However, being able to continue other traditional practices and ways of life was a significant way of coping with the stress of a new place, continuing one's sense of self-identity, culture, and connection to home, and forging new spatial relationships. In this manner, the girls and women were developing and maintaining attachments to multiple places simultaneously and enhancing a plurilocal and transnational sense of self-identity and belonging.

\section{5:4 Key learnings}

Returning to the research sub-questions introduced in Chapter One, it is clear that sensory memory plays a key role in shaping participants' feelings of place-attachment and wellbeing in Nelson. Because memory is embodied and relationally (re)constructed through everyday multisensory experiences (Blunt, 2003; Drozdzewski et al., 2016), individuals cannot simply forget or discard their former homes and identities, as they cannot separate themselves from the lived experiences and instinctive habits and routines which make them who they are. Thus, the aim of resettlement should not be to encourage former refugees to simply start over and create attachments to a new place, but to enable them to mediate between past, present, and future experiences, needs, and desires so that they can maintain valued aspects of their identity, manage grief, and regain a sense of safety, stability, and happiness in new places (Jones, 2011; Rosbrook \& Schweitzer, 2010; Sampson \& Gifford, 2010). Rishbeth and Powell (2013, p. 170) claimed that, "Theories of migration and place attachment need to encompass a flexible understanding of past and present landscapes, one which allows for ongoing development of transnational identities and a "multiplicity of trajectories" (Massey, 2005, p. 55) at a range of national and local scales".

In considering the places and homes from which former refugees have migrated, and to which they may still feel attached, I agree with Morse and Mudgett (2017) that more attention needs to be given to the non-human elements of place. For individuals with rural 
upbringings, such as the Chin and Kayan participants, the feelings of happiness evoked through a view of the mountains or the joy of collecting fruit from the forest may be equally as important as the value derived from one's sociocultural relationships or a relationship with a house or neighbourhood. There is also a need to consider the temporal rhythms, cycles, routines and paces-of-life with which individuals have become familiar in the past (Edensor, 2010; Harvey, 1994; Lefebvre, 2004; Potts, 2015; Seamon, 1980); and with which they may feel out-of-sync in new places. This point is particularly significant as place is not static, and participants were not missing a simple snapshot in time, but rather a unique rhythmic flow of events which brought a degree of structure and meaning to their lives.

Finally, it is important to recognise that reflecting on the past is not always regressive or passive, and for former refugees it is not necessarily pathological or traumatic. Through the generation of hope (Pain \& Smith, 2008; Wright, 2008), and the (re)imagining and (re)enacting of familiar and homely practices in new places, both nostalgic and fearful memories have the potential to be productive and transformative (Blunt, 2003). Thus, for some individuals, the best way to enhance feelings of wellbeing in a new place may not be through therapy or counselling, but getting outside and getting one's hands into the soil, or letting one's body yield into the familiar and comforting rhythm that comes from walking in the mountains. 


\section{Chapter 6: Therapeutic-Feeling Places and Solitude}

Participants' initial shock of displacement and ongoing nostalgic and fearful memories of home were compounded by other 'scary' sights, sounds, smells, tastes, textures and feelings in a strange new city. Whilst they also mentioned having positive experiences in Nelson, the girls and women tended to avoid the Central Business District (CBD) and instead frequent therapeutic-feeling places such as homes, gardens, parks, beaches, and spiritual places which made them feel safe and happy. They also appreciated taking 'time out' from society to spend private time alone or with their family, and framed these intimate solitary experiences as central to their wellbeing. Drawing on geographic theories of therapeutic landscapes (Conradson, 2005; Gesler, 1992), and psychology research on solitude (Long \& Averill, 2003), I argue for a more balanced approach to refugee wellbeing in this chapter, whereby urban stressors and the difficult work of resettlement are tempered with moments of rest and rejuvenation (Phipps, 2019b), and greater opportunity for emotional, physical, cognitive and spiritual renewal (Burger, 1995).

\section{6:1 Developing a sense of Nelson}

\section{'It is scary for us'}

Nelson City is often described as a peaceful picturesque place to raise a family or to retire. However, as demonstrated in the previous chapter, it can also be a scary place. In addition to feeling out-of-sync with their surroundings and over or underwhelmed by a new paceof-life, participants struggled with the sound and tempo of a foreign language. Ngai Ciang Sung said that when she first arrived in Nelson, "I don't really understand the language. So hard for me. I just only know yes and no". Ngun added that, "We [my family] are a little bit scared because we can't speak English and we don't understand English, so it is scary for us". When Ngun's daughter Dawt (who acted as our interpreter) relayed this comment to me, she claimed that a lack of English had led to some significant misunderstandings. She said, "When they [my mother and siblings] arrived [in New 
Zealand] they miss[ed] the aeroplane. So, they just stay in Christchurch airport and we are so worried, we are so scared!"

As Ngun indicated, speaking a foreign language was equally problematic to listening to one. Anthropologists Trnka, Dureau, and Park (2013) claimed that, in addition to a sense of seeing or hearing, individuals also have a sense of being seen or heard by others. For the girls and women, the fear of being seen or heard as a foreigner or someone 'different' was entangled in their memories of ethnic and religious tensions in Myanmar. Zai Zai said,

We are Christian, but ah, not-Christian people really hate, they really hate Christian people. So, I don't want to go in that place ... I mean other place, other city [in Myanmar]. When I visit another city ... how can I say ... we are different skin and look like different face. So, when they see us, she will, they know she is Christian. And they don't want to talk.

These prior discriminatory experiences meant that participants were acutely aware of sounding, looking, and feeling different as they settled into Nelson, and were understandably concerned about how they might be perceived and treated by local residents.

Whilst Nelson does not have the same level of violent ethnic discrimination which plagues Myanmar, there remains an unpleasant underbelly of racism directed toward 'foreigners'. During a conversation with a Māori man whom we encountered during Mu Pli's interview at ANZAC Park, he asked us if we would leave the park and go somewhere else. In response, a second man claimed that we had as much right as anyone to be in this space, and we agreed that we would stay and continue our mapping. Noting Mu Pli's unique Chin features, the first man then asked her where she was from and repeatedly questioned whether she could understand him, while referring to me as sister and praising the pounamu (traditional Māori greenstone pendant) that I wore around my neck. In this manner, he positioned himself and I as kin and Mu Pli as an outsider. These aural and visual reminders of one's difference are not problematic within themselves, though processes of social differentiation which place greater value on particular ways of sounding or looking can lead to discrimination (Howes \& Classen, 2014; Law, 2001). When such 
sensory norms are then connected to ideas of nationhood and who belongs in a particular place or not, xenophobia can emerge.

Ten out of the eleven resettlement practitioners whom I interviewed mentioned that they had witnessed or heard of incidents of verbal racism or aggression directed at former refugees. One practitioner claimed that, "A lot of it is by young people. I mean, there is an anti-refugee feeling out there anyway, like 'you're taking our jobs', 'go home', 'we can't house our own' - that sort of stuff". Elizabeth said, "Sometimes young people they don't like Asians, so, you know ... [I'm] scared ... I just don't feel [safe] but I don't hate them. Because, when you were young, everyone does silly things. I don't hate them, but I feel unsafe”. A practitioner from Victory Community Centre also added,

I think another difficulty in a real community way is local New Zealanders being uncomfortable with people of other ethnicities and particularly with people who have English as a second language, or when their English is almost non-existent. An example of that is, we have a playgroup here and in the last few years we were really conscious how many Burmese have moved on from the formal refugee process to being locals. And we invited them to come and be part of the playgroup, but the local people were finding that uncomfortable and so we had this incredible situation where the Burmese and the Nepali started coming with interpreters, but then the Pākehā parents stopped coming. Which was a real shame because we just thought this would be a wonderful opportunity, in an informal way, and everyone having a little child to bring them together ... so that's been really hard.

These uncomfortable, scary experiences could make former refugees feel unwelcome in the city and may act as a deterrent from participating in the public sphere.

'Scary cities' have been a key point of interest in Western geography. Along with the emergence of 'emotional geographies' at the turn of the century, there were a flurry of conferences, special issues, and books which explored geopolitical landscapes of fear and mistrust. In 2001 Bannister and Fyfe edited an Urban Studies Special Issue on 'Fear and the City', and in 2003 Shirlow and Pain edited a Capital and Class Special Issue on 'The Geographies and Politics of Fear'. A series of sessions on 'Scary Cities' was held at the 2008 annual meeting for the Association for American Geographers in Boston, Massachusetts, which led to England and Simon's special issue in Social and Cultural 
Geography in 2010. This fearful focus has become more nuanced over the past decade; interwoven with explorations of hope which draw out a more ambivalent and precarious experience of the city (Pain \& Smith, 2008; Wilson, 2008).

The nuances and complexity of everyday emotional relationships were further demonstrated by the girls and women, who often counterpointed their negative social experiences with positive ones. Elizabeth said,

[At] Nelson airport our sponsor [Red Cross volunteers] came and meet us and take us to a home. And they gave me, when I saw the place, our home, I was crying cause they, you know, our sponsor prepared all the living stuff, you know, and they said 'Oh, welcome home'. This is your home. And I feel very happy and there was happy crying. They welcome us like a family you know, and I didn't know them personally but the way they welcome us it feels like family and I felt very happy ... I feel very safe in Nelson and the people are nice. When you go out somewhere and get problem and say please help, everyone helps. They are looking for, they understand people very well. And um, they support a lot. So, I really like Nelson.

Jenavi added that, "Here in New Zealand people are very nice and very kind and I feel happy ... When we meet outside, 'hi, hi, how are you?' it's very nice".

Whilst these scary/safe experiences draw heavily on visual and auditory experiences, other sensory experiences also contribute to an individual's perception of the city (Law, 2001; O’Neill \& Hubbard, 2010). One of the resettlement practitioners said,

Do they [former refugees] feel safe? That's going to really affect how comfortable they feel. Compared to where they were on some levels, physical danger yes, but there may be other things they perceive as threats. That would be a really interesting topic to explore I think. Sensory issues around safety.

In addition to the concerns around language, participants were worried about the uncomfortable smells, flavours, textures, and visceral experiences of New Zealand cuisine. Little Ruth said, "At the airport I don't really like the sausage and pie. They [the Red Cross volunteers] gave it to me, but I don't like it". Meanwhile, Elizabeth found it strange that New Zealand food was presented on a plate as separate items, rather than being mixed together and served on rice. She said, 
Like, you know the beef? Roasted beef and beans here, and food here [shows everything separated out]. [It's a] different style of eating ... And no rice. Without rice, how are we going to eat it?!! Yeah, like in Malaysia we eat fried chicken or curry, but no curry or chicken [when we first arrived in New Zealand], just beef with the gravy and [it's] very different you know.

Sui Sung added that,

[New Zealand] is very difficult for food. First time I come and the Auckland Mangere camp I don't like for eating, it's very difficult! Like only rice and meat and other [kind of cooking] very difficult! Eating is ... ah, I'm crying, I'm hungry, I don't like ... I'm not wanting. Very difficult because I'm pregnant with Samuel. Too difficult for the eating. I don't eat for one week. Only milk, only drinking milk, and I'm vomiting too. Only drink milk ... A whole week! Very difficult. It's [the] taste, the smell I don't like.

Whilst unfamiliar tastes and smells can be troubling for any individual arriving from a new country (Kale et al., 2019; Law, 2001; Longhurst et al., 2009), participants also highlighted specific culinary challenges for women. Through connecting her negative food experience with her pregnancy, Sui Sung demonstrated that nuanced perceptions of place are further influenced by biology and unique physiological changes. Sui Tin Men also said that the smell of pesticides made her feel nauseous when she was pregnant, and she didn't like the way that chemicals changed the taste of the produce.

The women also had trouble with iron deficiency - a prevalent female health issue in New Zealand (Southern Cross, 2019). Zai Zai said, “When I went to New Zealand, I have to go to hospital for a blood test. Most of our Chin women, are really low iron they said. Because we can't eat red meat ... Lots of people we can't buy because we don't have enough money for red meat". This deficiency resulted in feelings of fatigue and exhaustion, further impacting the women's negative embodied experiences and impeding their mobility and access to, or enjoyment of, the city.

Whilst each sense alone may appear innocuous - a different accent, a feeling of being judged or threatened, difficult smells and flavours, or a sense of fatigue - the combination of unfamiliar, unsafe, and uncomfortable stimuli may be overwhelming and individuals may retreat to places which feel safer (Coughlan \& Hermes, 2016; Sampson \& Gifford, 
2010). For many of the research participants, this meant withdrawing from the Nelson

CBD. One of the resettlement practitioners said,

You walk down Trafalgar Street and you don't see, I mean how many ethnic people do you see? There are the tourists, but you don't see a whole bunch of Burmese or Nepali or Bhutanese or whoever walking down there. People are constantly surprised that we are the third largest diverse town in New Zealand.

This lack of public visibility had raised concerns for practitioners that former refugees may be isolated in their homes and lacking access to important institutions and support networks (Rosbrook \& Schweitzer, 2010). Yet, participants were still accessing the services they needed or liked in the city. Mai Aye Aye enjoyed going out for Thai food and Jenavi said, "I like to go shopping and like modern looking place ... Nelson, Richmond. I don't go to much shopping, just sometimes". The girls and women also felt comfortable in their local suburbs of Stoke and Victory (Toi Toi), both of which have a range of accessible public amenities. Thus, whilst former refugees were not always visible in the $\mathrm{CBD}$, that is not to say that they were not engaging in urban life or participating in the public sphere, but rather that certain urban areas felt safer and more accessible than others.

\section{'In the one suburb is good for me'}

Participants claimed that they felt comfortable in the suburbs because everything felt close. Sui Tin Men said, "I am happy living in Nelson ... not in Auckland, it's too big. It takes too long to get places. Nelson is good because everything is close and not far away”. Elizabeth added,

Yeah, you know I really like my house, the place. Because close to school, and also close to hospital, to my doctor. In the one suburb is very good for me. When I have emergency, I can ride to hospital, you know, and I think it's important that, how to say, close to live with the shopping... You know when I was younger each village [in Myanmar] was one mile or two mile we were walking.

Because of the limited infrastructure and ongoing warfare in Myanmar (Schwab, 2013; Smith, 1994), long-distance trips had previously left the women at the mercy of soldiers and storms and taken a physical toll in a proprioceptive and interoceptive sense, where muscles flexed and tightened and bodies became weary under the weight of bags and 
children. In contrast, the girls and women found living close to important institutions and social services in Nelson to be safer and less strenuous on the body. This closeness was particularly important for those individuals who couldn't drive, or who found the busy roads to be overwhelming and preferred to walk.

In their refugee resettlement research with Karen participants in Calgary, geographers Wood, McGrath, and Young (2012, p. 27-28) claimed that, "There is a relationship between physical and community infrastructure and the emotional attachment of refugees to their new home ... The geography of the city matters, particularly the proximity of public amenities and the ease of mobility". In her study on place-attachment and mobility in Göteborg and Umeå in Sweden, geographer Westin (2015, p. 734) also claimed that,

Most people's daily lives are fairly local; most people live with or near their immediate family (partner and children), and their children's daycare and school are often in the neighbourhood or at least situated within the municipality. We do not travel too far for daily shopping or leisure activities, and work is often the most distantly located activity. Thus, important people in our life and important activities are local, which might be reflected in high place attachment.

The women claimed that being in close proximity to their children was also important. Elizabeth said, "If my children go to school too far away it makes me worry, are they going to be okay? Or, if something happens, emergency, I think how can I go there?" Whilst this desire for physical closeness may be an inherent part of motherhood, or parenthood, it takes on added significance when understanding that children in war-torn Myanmar are often not safe and parents may need to find their children and escape at short notice. Toi Toi was particularly attractive in addressing these concerns, as the kindergarten, school, and community center were located in the heart of the neighbourhood and easily accessible from many of the women's homes.

Whilst strong neighbourhood attachments have been shown across a diverse range of people, a couple of studies have indicated that women have a stronger sense of territorial belonging or attachment to local residential areas than men (who are generally more mobile) (Gustafson, 2009; Westin, 2015). Because the majority of the women in this study were stay-at-home mums, they did not travel into the CBD for work and their daily 
routines centered around shopping and school drop-offs or visits with neighbours in their suburbs, making these local areas more familiar and comfortable.

Yet neighbourhoods can also feel scary, and as a lower socio-economic area, Toi Toi has a reputation as the 'bad part of town'. During her interview, Sui Sung mentioned a spate of violent neighbourhood attacks which had changed the way that she perceived her street. She claimed that whilst she usually felt safe and comfortable at home, she was scared because her car had been smashed while it was parked on the roadside nearby. She said,

In my home I have no garage. This one is difficult to drive my car. It's broken ... Other people they [shows smashing motion], and I'm scared ... I know three or four people coming [shows smashing motion again] ... I'm only telling my husband, only, why I don't talk, I'm scared ... I'm looking out my window thinking nooooo! One hour, two hour, in the morning. Two or three times. I'm see it only one time but my car broken two or three times... I don't know who are their names the people, I'm not sure for the police. I dunno. But girl and boy ... they [laugh] 'hahahaha', BAM, ping! [Shows smashing motion].

Remembering the sound of cruel laughter, smashing glass, and pinging metal made Sui Sung flinch while she demonstrated how she had peered out of the window whilst trying to hide herself from danger in the shadows of her curtains. To avoid these scary experiences and the stressors of the city, participants would often seek out specific places which had a more relaxing therapeutic and rejuvenating feel. Primarily these consisted of the girls and women's family homes, though outdoor and spiritual places were equally as important.

\section{6:2 Seeking therapeutic-feeling places}

\section{The safety and comfort of home}

One of the main factors that made homes conducive to a therapeutic experience was a feeling of safety. Mai Aye Aye said, "Home is very important for me because it makes [me] feel safe and relaxed". Studies of home as a safe space have noted multiple pathways through which positive feelings of safety might develop, with one key aspect being a sense of familiarity. Because most people spend a significant amount of time at home, the house becomes a familiar place through which an individual can instinctively move and develop 
habitual rhythms and routines. In their study with home-care workers for elderly patients in the UK, health researchers Martin et al., (2005, p. 1901) claimed that, "In some circumstances the emotional significance and the physical familiarity of the home setting were seen as most conducive to patients' psychological and functional rehabilitation". However, familiarity and routine can also become monotonous and boring, and they continued saying, “ $\ldots$ at other times it was escaping the confining walls of the house".

The girls and women also felt safe at home because the people with whom they lived were familiar, and they had the opportunity to be close to their family and strengthen familial bonds. Iang Chin Sung added, "Outside I am happy too, but my house feels safe for my family and I am happy living here with my husband, children, and mother. Just eating, sleeping, and being inside". Participants in Sampson and Gifford's (2010, p. 126) study with former refugee youth in Melbourne also discussed the importance of being in close proximity to family. The authors claimed that,

Places of family togetherness featured highly in settlement landscapes and photonovellas reveal these as important sites for reaffirming relationships and for displaying cultural artefacts, family photographs and other objects of cultural familiarity that together create a sense of belonging and being at home.

This togetherness is particularly important for former refugees who have been separated from family members and systems of social support. However, it has also been noted that intimate family relationships are not always safe, and domestic violence is a significant threat within some former refugee households (Nelson Multicultural Council, 2012; Ward et al., 2018), as well as in the households of other New Zealanders (James, 2010).

Proximity to household amenities was also an important aspect of safety and comfort. Sui Sung said that she felt safe in Nelson because her toilet and shower were located inside her house (compared to the outdoor bathrooms in Myanmar) and she no longer had to walk out in the dark at night to do her ablutions. Iang Chin Sung also said that in Myanmar, "The toilet and bathroom were outside and the water was cold, especially in the winter. We would boil the kettle on a fire to heat the water. Having the bathroom inside in New Zealand houses is better". Mu Pli added, "The water you know, supplies are so much better here, you don't have to go all the way to a public place to use it". 
Several of the women stood up during their interviews and acted out methods of how they had collected water and washed in their villages, demonstrating how they would hold a cold garden hose to spray themselves, and use their upper-body strength to hoist a bucket of water up from a well before heating it on the fire. They agreed that showering or bathing indoors in Nelson was an improvement as the water felt warmer upon their skin and this method was less taxing on the bodies of the elderly and infirm, who might struggle to lift a heavy bucket. Thus, there was a general feeling of comfort because of the structural way that Nelson houses enabled basic needs to be met with minimal physical exertion.

Participants also spoke about the importance of electricity in helping them to feel safe and comfortable at home. When Mu Pli reflected upon her life in Thailand she said, "[Now] I think I would be so scared to live there because I got so used to electricity here. It's dark back there! Electricity also offered sources of heating to which participants had previously lacked access. Zai Zai said, "In Myanmar we are really poor ... Poor means when we get really cold we can't buy warm clothes". As previously mentioned, the New Zealand climate is comparatively cooler than Myanmar, and the use of electric heat pumps and heaters in New Zealand houses thus provided a welcome sense of warmth for the women and their families. However, these positive warm feelings could not be taken for granted either, as New Zealand has significant issues with cold damp mouldy housing which can be extremely detrimental to wellbeing (Evans, 2006; Nelson Marlborough Health, 2016).

Homes (and cities) could also feel uncomfortable due to people being messy and the inevitable build-up of dirt and decay. Reflecting on her experience in Myanmar, Zai Zai claimed that, "The first place I don't want to go to is the ... dirty place. Some place is really dirty, a lot of rubbish in the city”. This dirt and mess could make participants feel stressed or lead to them avoiding otherwise enjoyable places. Similar correlations were found in Saxbe and Repetti's (2010) research, where clutter in the home was a significant source of stress for married women, and Sampson and Gifford's (2010) research, which found that former refugees were actively seeking out clean and beautiful places.

Despite the many factors which could potentially prevent homes from feeling therapeutic, the girls and women described their experiences at home as 'happy'. Sui Sung claimed that, "[In] my home I am very happy”, and Mai Aye Aye wrote on her street map, "Home 
is very important for me. Because very warm and very good view. My children also happy”. Drawing on Psychologist Fredrickson's (2004) work, geographers Scoffham and Barnes (2011, p. 537) claimed that,

We take happiness to mean a state of flourishing that involves a sense of personal fulfilment within a shared moral framework. This definition recognises that happiness is a relative rather than an absolute notion. It occurs in various environments and can arise from a variety of stimuli.

Feeling happy is significant in terms of enhanced mental health and wellbeing, as positive emotions can influence individual thoughts and behaviours. Scoffham and Barnes also claimed that, (p. 540), "People in a positive state of mind show an increased preference for variety and a greater willingness to accept difference. Exploration, imagination, inquisitiveness, playfulness and the ability to make relationships all appear to be enhanced". Fredrickson (2004) further argued that positive emotions enable individuals to develop positive thought patterns which can equip them to cope with stressful or traumatic events and circumstances and generate a feeling of wellbeing, which in turn further enhances a feeling of happiness.

\section{Healing and rejuvenating land, sky, and seascapes}

Participants also spent a significant amount of time relaxing, exercising, and playing outdoors. Zai Zai said that she enjoyed, "Thinking under the tree and with fresh air", and Mu Pli wrote on her body map that she liked the wind blowing because there was a "nice breeze". She also wrote on her street map that she enjoyed playing volleyball and football at Victory Square. Similarly, Little Ruth wrote, "I do like to stay outside [in] my garden and have some oxygen and look after my three little brothers", and added, "My favourite sports is volleyball and football". The older women were not as interested in group sports, but enjoyed walking in the hills, cycling, and taking their children to play at the playground. Mai Aye Aye said, "I like ride bike because it reminds me of Myanmar", and Ngai Ciang Sung said, "Playground is important for me because I can spend time with [my] children. Hearing the children makes me happy”.

Ngun also claimed that she felt, "Happy in the beach ... it is relax[ing]", and on her street map Elizabeth said that she liked to go fishing in the Kahawai season and enjoyed 
dragging the fishing line through the water because it 'feels good'. Zai Zai added that she liked to go camping because, "We play with the beach and looking at the crab. My kids like to play with the mud". Meanwhile, Sui Sung claimed that when she felt upset, gardening helped her to feel better (Figure 6:1), and Jenavi wrote on her body map that she enjoyed the feeling of the compost and the water when gardening. Elizabeth also wrote that she enjoyed making fertilizer and watering her plants.

These multisensory multi-textured experiences are often overlooked in the literature on therapeutic green and blue spaces, which tend to prioritise the visual aesthetics of place. Geographer Brown (2016, p. 307) claimed that,

Even the term 'greenspace' implicitly and narrowly privileges the visual by framing the environment through colour, when the materialities of environments making them green (or indeed brown, blue, grey, purple, and so on) often include patterned stone or hardcore, undulations of earth and tree-roots, pockets of mud, puddles, and carpets of vegetation or concrete, which have to be bodily negotiated.

Yet, multisensory textured experiences were significant in connecting the girls and women to their former rural agricultural practices from home, as well as connecting them to a new place and ensuring that they remained active and healthy.

One of the resettlement practitioners said,

I don't think we make nearly enough use of the wonderful natural landscapes that we have here [in Nelson], in normal health. It's a fantastic resource and it's really underutilised and I would imagine, if people had the opportunity to get down to Rabbit Island in the car and have a picnic with some friends for two or three hours, kicking a football, kids running round, and swimming, that could just be hugely valuable because it connects them with the ground, with the sea, with a place they feel relaxed in and safe in and it's fun. Fun and laughter are really important, and getting their feet on the ground, into the soil.

Connecting with the ground, or what this practitioner referred to as 'grounding', is a practice which has recently been adopted as a controversial form of environmental therapy (Chevalier et al., 2012). Grounding (or earthing) is based upon the theory that the human body is electrically conductive, and that, by having direct contact with the earth's surface, 
the body reacts to the presence of environmental electric fields by absorbing the grounds free electrons. This reconnecting with the ground is said to improve sleep and reduce pain, amongst other physical and psychological health benefits.

Whilst this theory is heavily debated by skeptics (McDuffee, 2015; Novella, 2012), it has inspired local practices of beach-walking as part of a pilot initiative called 'Stress Busters'. Stress Busters was founded by two of the practitioners in this research, a local occupational therapist and psychologist, and worked closely with Nelson's Colombian community to combat the high levels of psychological distress and ill-health within this population. In a conjoined interview, the practitioners said, "We do Stress Busters here in different forms. We provide mindfulness and muscle relaxation etc., etc., but we also do beach walking now ... After every course, every time, people rate beach walking over and above, as more effective than anything else". Thus, the simplicity of getting outside, being active, and connecting with the ocean or the earth is significant, and regardless of the scientific cause of feeling relaxed, participants expressed a positive emotional outcome.

Beautiful views could also be therapeutic. Reflecting upon her first impression of Nelson, $\mathrm{Mu}$ Pli claimed that,

The first thing was when I was on the plane. I just saw the landscape and it was all so square. When you look down, it was so beautiful. It was so square and neat and beautiful ... I just looked down and was like wow! And the ocean! I could see around the ocean and it was so big, I was like wow! Look at the little land there ... I don't think I see the beach or the ocean [in Thailand].

She also said, "Especially on a sunny day I'll look up at the clouds, and in Thailand I used to do that too, just spend time looking at the clouds. And, I dunno, it makes me happy when I look at the clouds" (see Figure 6:2). Meanwhile Sui Tin Men mentioned that she had never before seen snow and that she felt drawn to the stunning vista of the Tasman Bay mountain range (which stretches from Lookout Range due West and continues northwards towards Mount Evans). She said, “[In] 2006 I came [to Nelson] and [it was] very cold, July. Very cold in Nelson and it look[ed] different. White colour on mountain. And I look, morning time, white colour, oh! Different. Chin State no snow. Mountain all white colour and 'Oh! Nelson!','. 
Figure 6:1: Feeling Better in the Garden

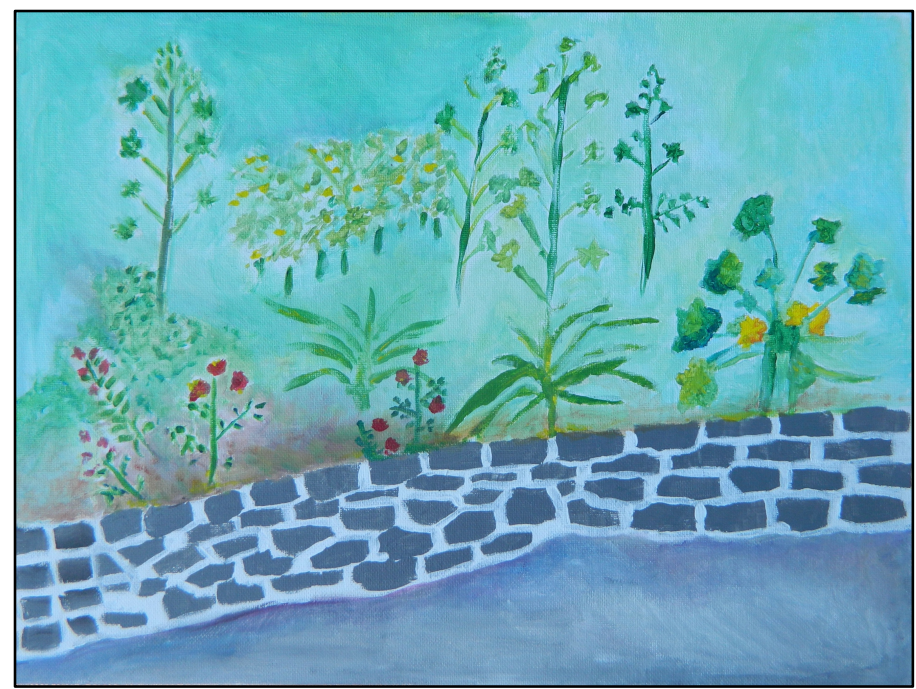

(Source: Painting by Sui Sung, 2019)

Figure 6:2: Watching the Clouds at ANZAC Park

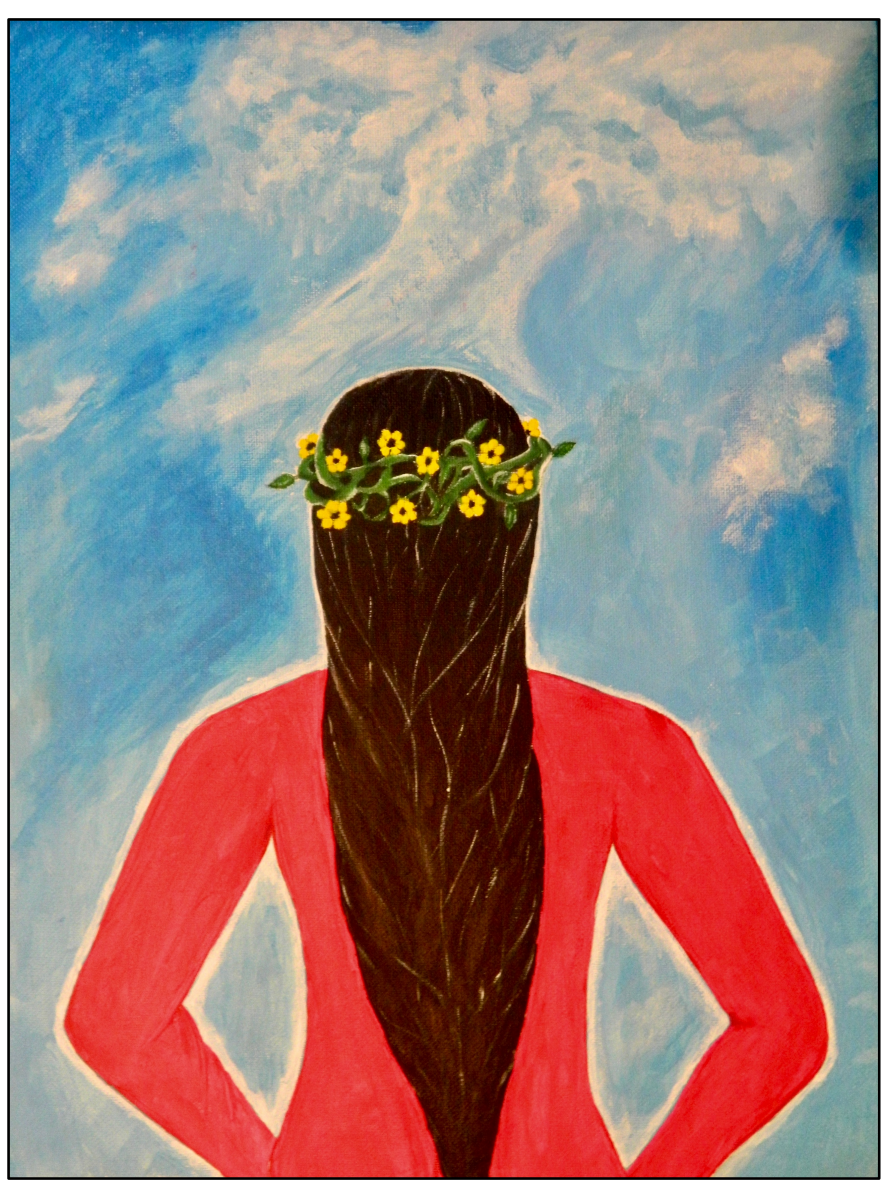

(Source: Painting by Mu Pli, 2019) 
Researchers in environmental psychology have claimed that access to pleasant outdoor views can enhance everyday wellbeing and recovery from illness (Moore, 1981; Ulrich, 1984; Verderber, 1986; West, 1986). Recreation theorist Hammitt (1982) and Environmental psychologist Kaplan (1978) also argued that, when individuals are in places which they find naturally beautiful or fascinating, they are not working as hard to stay focused or motivated and are therefore more likely to feel alert, energetic, and enthusiastic. Hammitt and Kaplan's arguments were also supported by environmental psychologists Hartig and Staats (2006) study with Swedish University students, which found that fatigue was alleviated and attitudes were more positive when participants were walking in pleasant outdoor spaces. Thus, for former refugees who may be feeling anxious, stressed, or worried, these views could play a prominent role in their ability to heal from pain and trauma and feel rejuvenated after stressful urban encounters (Coughlan \& Hermes, 2016).

Views were also important in helping the women to feel connected to the wider world beyond Nelson City. Ngai Ciang Sung claimed that she enjoyed walking up the 'Centre of New Zealand' (a relatively steep half-hour climb to the nation's geographic centre and a popular scenic lookout point). She said, "This place [the Centre of New Zealand] is not important but just when I'm [walking up there] it makes me happy. I like the views. Mountain is very important for me because it make me feel more connected to everything”. She explained this feeling of connection further by saying that when she stood on the ground (at sea-level) she could only see her immediate surroundings, but when she climbed a mountain she could see the horizon; a vision of endless sky, land, and sea that connected her, and Nelson, to the world and everything in it.

This sense of connection was important to participants who had spent a significant portion of their lives living in remote villages and isolated refugee camps. Mu Pli said,

[In Thailand] I used to think we were the only ones on the planet because we are so isolated from other countries. Although we get indications, you know, for me personally I didn't think there are so many more people and so much more places and countries in the world. I was really surprised when I learned that cause all I ever saw were the people around me, my village, just the family, relatives, and between the Big Refugee Camp 1 and Nai Soi Village that is where I mostly went back and forth. 
Thus, expansive views helped participants to feel less alone in a new place, whilst also enhancing a sense of curiosity about what or who else was out there. A participant in geographer Bell et al.'s (2015, p. 62) UK research on coastal blue space also mentioned this sense of connection to 'something bigger' and said, "So to have that space and realise that there's a bigger thing out there than you ... It kind of puts things into perspective”.

Whilst views of distant horizons could evoke a sense of curiosity for the unknown, so too could the easily overlooked sights, sounds, and smells of local flora and fauna. Jenavi claimed that the colour green was significant (see Figure 6:3), and explained that,

New Zealand tree is, I think twelve months, some leaves is always green. It's very good. Myanmar is, some season all leaves are down so is looking not nice. But here always is very green. So, I love so much this green. When leaf grow up again, this time is very nice to look.

She added that she enjoyed listening to the unique New Zealand birdsongs, saying, "Some birds sound the same but most sing different in Myanmar”. Meanwhile Mai Aye Aye claimed that, "I like to go to [ANZAC] park because it makes me happy. I like the shade under the tree. I like spring-time because the flower is pretty and smells nice" (see Figure 6:4). Mu Pli added, "We don't have many flowers back in Thailand, so flower is what I'm intrigued to. Like really interested in. I'll just stare at the flower, and I don't mind that". Meanwhile, on her street map, Little Ruth wrote, "The park is my favourite place to play or draw something that I [find] interesting”.

Philosopher Zuss (2012, p. 122) claimed that curiosity occurs through questions, which "arise from the filaments of our senses". Geographers Phillips, Evans, and Muirhead (2015, p. 2346) added that, through the act of being curious and exploring one's surroundings, "Ordinary objects, now the subject of interest, and fascination, become extraordinary, even enchanting". The notion of enchantment has also been explored by political theorist Bennett (2001) and cultural geographers Geoghegan and Woodyer (2014). Bennett (2001, p. 5) claimed that to be enchanted is, "To be struck and shaken by the extraordinary that lives amid the familiar and everyday ... enchantment entails a state of wonder . . . a momentarily immobilizing encounter; it is to be transfixed, spellbound". 
Figure 6:3: Enjoying the Green Leaves in the Hills

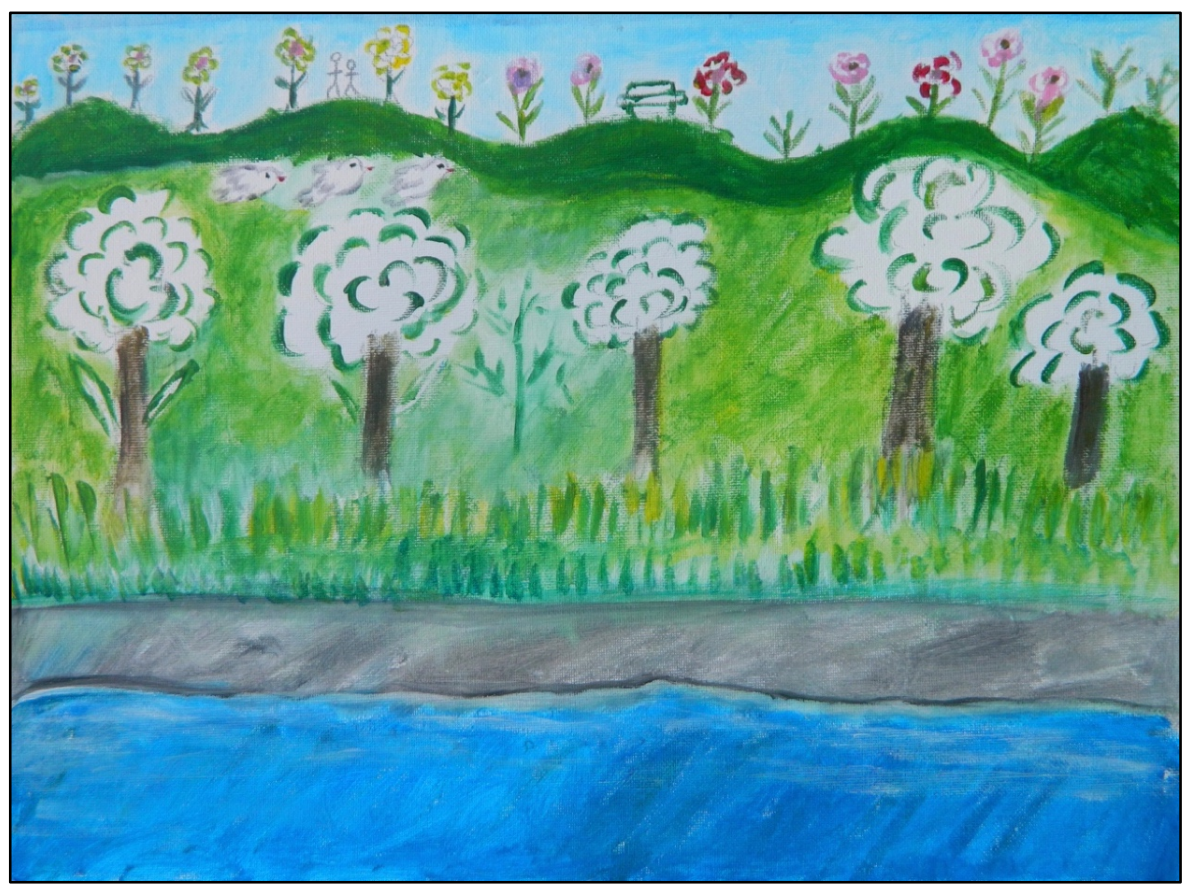

(Source: Painting by Jenavi, 2019)

Figure 6:4: Spring-Time at ANZAC Park

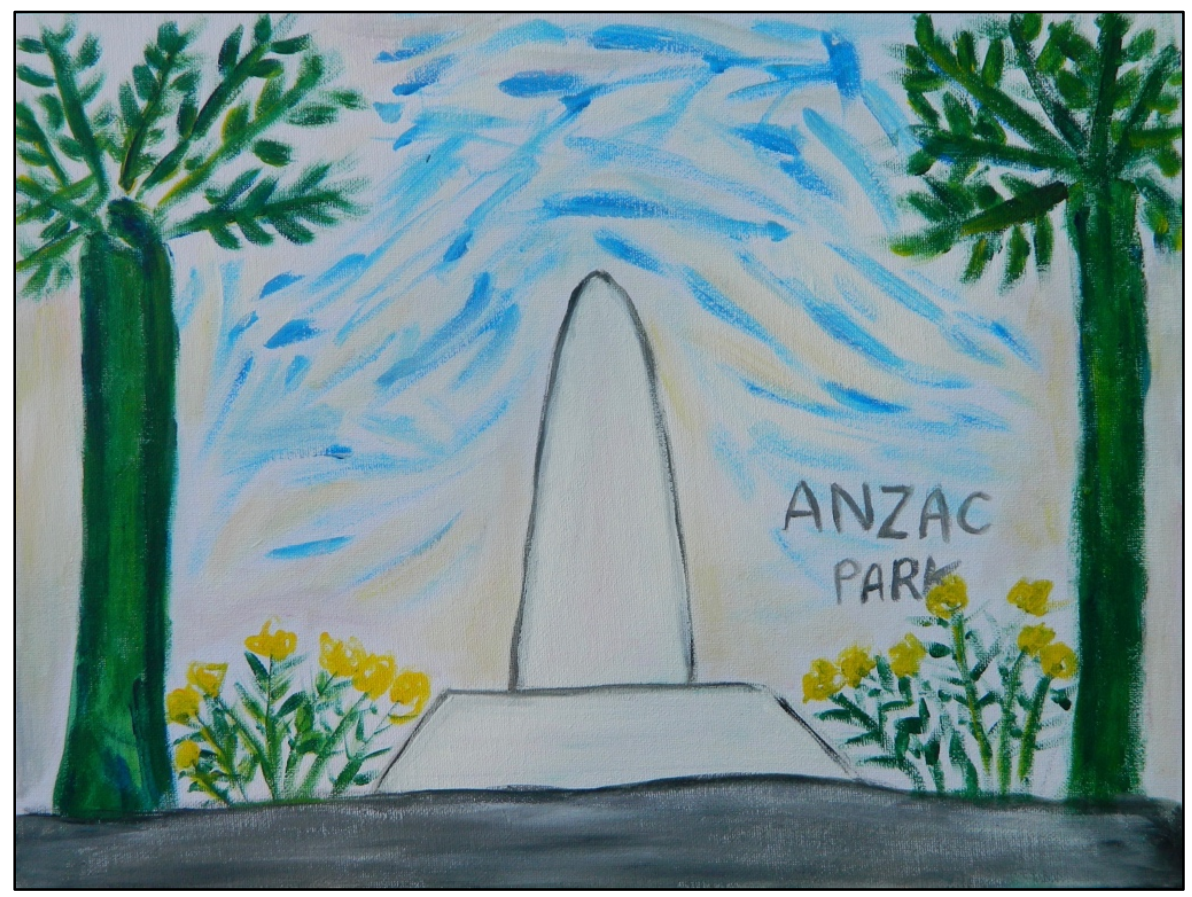

(Source: Painting by Mai Aye Aye, 2019) 
Both curiosity and enchantment occur during the disruption of that which has come to be taken-for-granted, and have been framed as a recasting of alternative realities and new possibilities. In his research on sensing ruins, geographer Edensor (2007) claimed that the habitual nature of everyday rhythms and routines can desensitise people to their environment, and a break from these rhythms and routines can be reinvigorating and foster a feeling of delight. Geoghegan and Woodyer (2014) also suggested that adults often get trapped in familiar routines and lose the sense of wonder for everyday life that is exhibited by children. However, a break in routine (such as taking time out from the city) can reenchant individuals as they are exposed to new experiences. These enchanting interactions have significant value in terms of wellbeing, and Geoghegan and Woodyer (2014, p. 220) argued that, "Dwelling in the small and seemingly inconsequential often affords respite and strength in abject situations; fleeting moments that can accumulate and sustain hope in times of real difficulty".

This enchantment with the birds, leaves, and flowers also highlights the role that nonhuman entities play in the co-constitution of a therapeutic experience (Conradson, 2005; Gorman, 2017). However, as Gorman (2017) pointed out, human-animal interactions may not necessarily be therapeutic for the animal, and there can be multiple problems and risks involved when engaging with wildlife. Elizabeth wrote on her body map that she felt stressed by the bugs eating the vegetables that she had planted in her garden, and Little Ruth wrote on her body map that, despite Whenua Iti being a place to which she felt attached, there was the, "Smell of dead animals ... Sandflies bit on my body ... I hurt my ankle ... [and] I feel so freezing cold".

Not everyone will find outdoor places to be therapeutic and studies have suggested that places like parks and woodland can also have a negative effect on place-attachment and wellbeing. In Milligan and Bingley’s (2007) study of children's interactions with woodland areas in the UK, some participants felt uncomfortable due to the texture of the dirt and the sight of the insects. They also expressed concerns over being in a dark, dense, isolated area where they felt vulnerable and unable to access help or medical care if needed. Collins and Kearns (2007) also argued that the therapeutic value of beaches can be romanticised and not everyone will feel comfortable on the coast. They claim that potential risks to wellbeing in these places - such as drowning - may be overlooked. 
Little Ruth wrote on her street map, "I'm not interested to go at the beach because it too hot and the water are salty". Coming from a landlocked state in Myanmar with only freshwater access, the saltiness of the ocean was often a point of disdain for participants. In addition to the strange texture of salt drying upon one's body, Elizabeth claimed that New Zealand fish were 'stinky' and Sui Sung said that New Zealand fish were, "Different for eating, because Myanmar is fresh water [and] this one is ocean, saltwater. Fish, mussels, everything tastes different".

Many of the participants were also afraid of the ocean because they couldn't swim. One woman said, "I like views. When we go on the hill and to look [at] the ocean, this makes me feel happy. But I don't like too much to go [to the] beach, just to look from away ... For me is not, we can't swim. So, for me is not happy to ... Just I go away and look from the mountains". At the time of our interview I didn't question her further; however, I later found out that her husband had drowned in a local fishing accident. Thus, whilst many people (including myself) take for granted the cathartic rhythm of the waves and soothing feeling of being enveloped by water, others may be apprehensive of such sensations.

\section{Spiritual places}

The girls and women also spoke of the value of spiritual places. Sui Sung said, "I' $m$ very happy when I pray in my house. Sometimes you know I'm very sad, sometimes I'm very worried. This time, I pray and cry and pray and cry and pray then am very happy". This cathartic process of crying and praying involved an inward-focus where Sui Sung was somewhat oblivious to external sounds, movements, and sights and overcome with the proprioceptive and interoceptive feelings of her body tensing and convulsing with sobs, and the heat and taste of salty wet tears upon her cheeks. During our interview she cupped her hands in front of her, kneeling and gently rocking as she talked about how she prayed.

Elizabeth also spoke about her relationship with God as cathartic and healing; however, the places and embodied experiences through which she connected to the spiritual realm were different from Sui Sung's solitary crying and praying at home. She said, "I feel like when [I] sing to God loud, like God can hear me. It makes me feel good and strong; and feel in my heart safe, blessed and healthy. I have no worries; God can heal our heart". She painted a picture of members of her church congregation singing and dancing together 
(Figure 6:5), depicting a loud, energetic, emotional experience which viscerally swept her up in the moment and helped her to feel invigorated. In this manner, healing for Elizabeth was as much a social experience as it was personal or spiritual, with the painting expressing a sense of rhythm and synchronization that exists between individual churchgoers, connecting each person present to something greater than themselves.

Whilst Mu Pli did not specifically mention the need to heal, she too claimed that feeling connected to a religious community and place of worship was important. She said,

I went to church and listened to gospel and that's something I believe in right now ... I'm just really connected to this you know. And whether by going to church and living the life of a Christian, I think that, especially emotionally and spiritually I am living a healthy life basically. I believe that. Going to church like that will help me with that you know. Church is like my strong foundation.

Christian and Muslim immigrants in geographers Agyekum and Newbold's (2016) study in Ontario expressed a similar sentiment, and claimed that an enhanced sense of connection to people and to God could decrease a feeling of loneliness and help them to feel as though they were part of a family or community. Other studies have suggested that this sense of spiritual/social connection can decrease negative emotions, increase positive emotions (Koenig et al., 2012), and foster feelings of empowerment and greater psychological stability (Oman \& Thoresen, 2003). Thus, religious or spiritual experience has the potential to aid recovery, but also to develop strong supportive and caring relationships - both important aspects of wellbeing. However, like homes and outdoor areas, these places of worship are not therapeutic in and of themselves and their value cannot be assumed.

Because therapeutic experience comes from a relational encounter between individuals and other people or non-human entities (Conradson, 2005), it is important to consider how everyday spiritual encounters might transform ordinary urban or rural environments into therapeutic places. Whilst Ngai Ciang Sung said that she enjoyed going to church and singing, dancing, and listening to sermons, her painting depicted her reading the bible at home (Figure 6:6). She said, "When I have time I read the bible and spend time with my family". As mentioned above, Sui Sung also liked to pray in her home, and added, “Sometimes I'm sad, I go to my garden. Pray in my garden much better. Much, much, 
better". In this manner, the women were taking control of their own wellbeing through transforming homes and gardens into spaces for spiritual engagement and reflection.

Other former refugees in New Zealand have also talked about practicing religion at home or in the city, as part of their normal daily routine. In a 2015 pilot painting workshop that I facilitated in Wellington, five Ethiopian and Eritrean participants of mixed ages, genders, ethnicities, and religions painted pictures and discussed ideas of what health and wellbeing meant to them. One Muslim man said,

Spirituality is very, very important. That's how I was brought up, praying five times a day ... Your body must be clean all the time, you have to wash your legs, hands, and faces when you want to pray ... it helps my body, it feels like a nutrition. It fills my body and my brain and very much helps my wellbeing and health.

Similarly, in geographers Dyck and Dossa's (2006, p. 699) study with female migrants and refugees in British Colombia, the authors also claimed that religious practice was woven into everyday routines and chores. They said,

It is through the temporality and spatiality of prayer, embodied in women's practices, that we see women re-inscribing the landscape - extending the healing practices of a 'healthy home' into public space. As they offer prayers in times of crisis, sadness or sickness in homes and neighbour-hood space, they further build the home and neighbourhood as healthy space—in material, social and symbolic dimensions.

The therapeutic value of everyday spiritual encounters is recognised in Māori health models (Durie, 1985; Pere, 1997); though remains neglected in New Zealand's dominant Western biomedical approach. This omission could in part be due to the dominant group of New Zealanders (including myself) identifying as atheist or agnostic (Stats NZ, 2019a). However, participants' comments and paintings demonstrate the intrinsic value of embodied multisensory religious practice in helping them to feel relaxed, rejuvenated, healthy, and connected to other people or places. Thus, the therapeutic value of crying, praying, dancing, singing, and purifying or washing one's skin should not be ignored, and may be particularly important for individuals who do not separate their personal and cultural identity from these embodied everyday religious routines. 
Figure 6:5: Listening, Praying, and Singing at Church

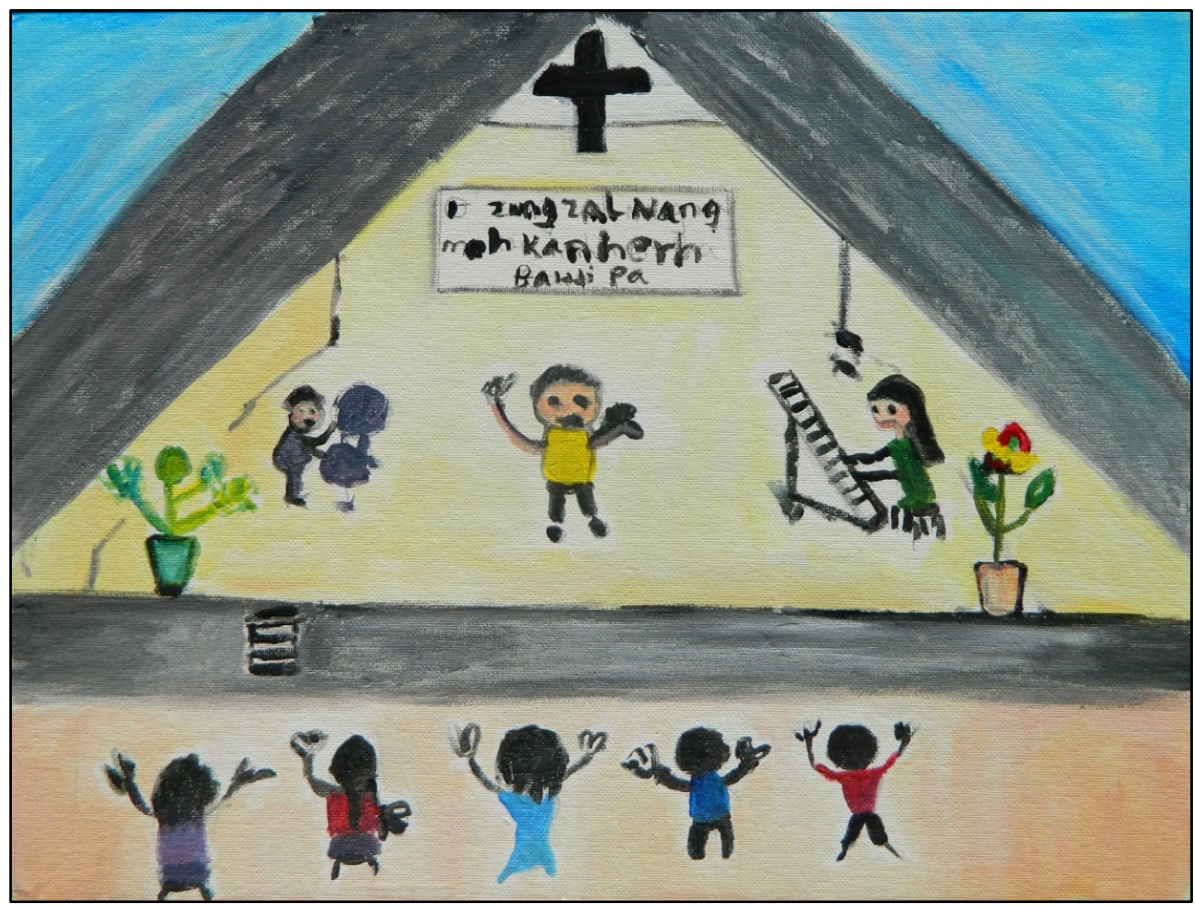

(Source: Painting by Elizabeth, 2019)

Figure 6:6: Reading the Bible at Home

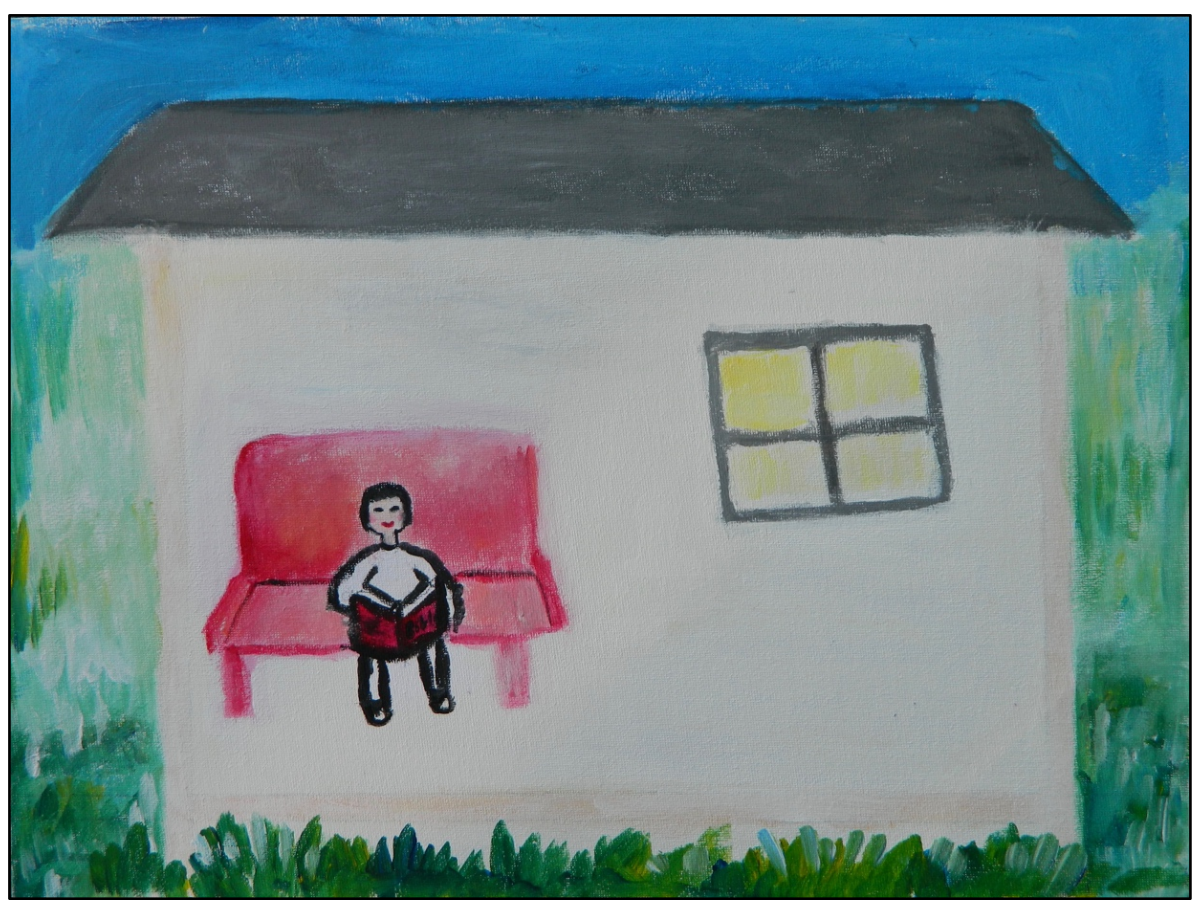

(Source: Painting by Ngai Ciang Sung, 2019) 


\section{6:3 A desire for solitude}

\section{'Just enjoying time being alone'}

Another important aspect of therapeutic places, which has received limited attention within the geographic literature, is the concept of solitude - or the positive emotional experience of spending time alone somewhere. Mu Pli said,

My most comfortable place is my home in my own room. Cause that's where I spend most of my time just being alone. Just look through social media or watching YouTube or singing sometimes, dancing on my own! And yeah, just enjoying time being alone in my own room when I have spare time.

Former refugee participants in geographer Biglin's (2020) research on therapeutic allotments also mentioned that they appreciated spending time alone, as did young people in geographers Milligan and Bingley's (2007) study on restorative places, and elderly individuals in Conradson's (2005) research with guests at a respite care center in Dorset.

Conradson (2005, p. 337) claimed that, in seeking therapeutic landscapes, "The opportunity to achieve a measure of change in one's everyday sociality has also often been significant. Many urban dwellers, for example, experience the relative solitude of rural settings as a favourable contrast to the rhythm of their everyday lives". Whilst he hints at the salience of solitude in everyday geographies, Conradson is one of the few geographers who have elaborated on the idea that time spent alone may be central to healing or other forms of personal and social development and wellbeing, although geographer Tuan (1976, p. 270) has also claimed that, "In solitude a person creates his own world; safe from another's gaze he seems to sustain the existence of all that he sees. All people need privacy; the degree and kind vary".

Positive experiences of aloneness have also been overlooked in refugee resettlement research, where the focus is overwhelmingly on social participation, contribution, integration, and connection (Askins, 2016; Blunt \& Dowling, 2006; Kale et al., 2020; Rishbeth et al., 2019; Strang \& Ager, 2010). Because there is such a strong political, economic, and social incentive to get former refugees active in their new community, seeking solitude or retreating away from the public sphere can be viewed as regressive. 
There is often a negative assumption that former refugees who are not visible in the city are suffering from trauma or not making an effort to contribute to their new society (assumptions which are generally inaccurate) (Ford, 2012); or, that due to the current climate of fear around refugees, they are being intentionally excluded by other residents (which unfortunately does occur) (Hynie, 2018; Kale et al., 2020).

These negative conceptualisations of being alone are further problematised in theories of 'social isolation', whereby an individual's exclusion from the sociocultural, political, and economic sphere has been shown to adversely affect their access to local resources, support networks, and opportunities (Rosbrook \& Schweitzer, 2010). Social isolation has also been linked to troubling or painful feelings of 'loneliness' and decreased individual and social wellbeing. Psychologists Long and Averill (2003, p. 22) claimed that, "In contrast to solitude, loneliness is generally seen as a negative emotional state which most people seek to avoid; it indicates deficiencies in the number or extent of one's social relationships".

Experiences of social isolation and loneliness are real threats to refugee wellbeing, and theories of social connection deserve the attention that they receive in current resettlement research. Indeed, my own research has frequently argued for greater relationship-building initiatives between former refugees and their new communities (Kale \& Kindon, 2020; Kale et al, 2019; 2020). However, the pressure to be socially engaged when one is dealing with significant disruption, loss, and overwhelming new environments can sometimes be too much. When I asked Mu Pli if she spent time socialising with other New Zealanders outside of the Kayan community, she said,

I'm not someone who likes to go out often ... and they [New Zealanders] tend to like to go out so much. It's not so bad but personally I think if I'm friends with someone, they will go out so much and invite me all the time and I will feel bad like rejecting saying, "oh no, I need time out".

This comment resonated with psychologist Burger (1995), wildland recreation theorist Hammitt (1982), and psychologists Long and Averill's (2003) claims that, when an individual is struggling with significant social and environmental stressors, being alone may not be viewed as a negative experience, but rather as a welcome form of relief. However, Long and Averill (2003, p. 30) also cautioned that, "The voluntariness of the 
episode [of solitude] is perhaps the single most important factor distinguishing between positive and negative experiences of aloneness". Thus, in understanding the benefits of solitude, spending time alone must first and foremost always be understood as a choice.

Whilst solitude or 'time out' is not talked about in Nelson resettlement praxis, practitioners also expressed an interest in pursuing this conversation. One practitioner said,

I think that the sense of taking time out and reflecting is a very Western thing, and I'd be really interested to see what people [the former refugee participants] say in regard to that, because I think having time to reflect and to centre yourself can be a luxury. And when people are tied up in life, and just getting through, and busyness ... I'd be really interested to see what people say about it, whether they take time out to reflect in that way.

Thus, in what follows I attempt to loosely define solitude and highlight the key benefits that participants have gained in seeking a moment alone somewhere.

In psychology and related social sciences, solitude has been conceptualised in a number of different ways. Most commonly this term refers to the physical distancing of oneself from other people (Burger, 1995; Conradson, 2005; Long \& Averill, 2003; Westin, 1967). For some individuals it may mean completely withdrawing from society and living a reclusive life in the wilderness, though often people will seek temporary moments of solitude in between social commitments and public events (Long \& Averill, 2003; Hammitt, 1982). How regularly an individual chooses to socially engage or spend time alone can depend upon a wide array of social, geographic, economic, and emotional variables, including how welcome they feel somewhere, their proximity to public spaces and access to transport, and the funds they have available to spend on social activities. It may also depend upon personal preference. Individuals with introverted personalities may be content on their own, yet those who are more extroverted may crave the stimulation and energy generated through intersubjective interaction (Burger, 1995; Conradson, 2005).

Solitude could also mean being alone in a public place where other residents are nearby but not necessarily interacting (Burger, 1995), or spending intimate time 'alone together' with a partner, family, or close friends (Hammitt, 1982). Thus, the aim of seeking solitude is not necessarily to avoid all human interaction (although this may be preferable at times), but 
rather to avoid the types of public spaces and social interactions that one finds stressful, with the intention of re-engaging with society when one feels comfortable doing so.

As suggested by Conradson (2005), solitude may also mean an escape from the rhythms and stressors of the city. When discussing wilderness solitude (time spent alone or alonetogether with others in remote rural places) Hammitt (1982, p. 480) claimed that,

One of the principle values derived from wilderness is escape - an opportunity for temporary release from the rules and pressures of everyday life. Solitude in this situation refers to escape and isolation from certain social structures and environments, rather than isolation from individual people.

Meanwhile, other theorists suggest that solitude may not only require an escape from people and pathological city stressors, but from all external entities, objects, and sensory stimuli, pathological or not. By attempting to block-out anyone or anything that may be considered distracting, individuals can become attuned to their inner senses and thoughts and more mindful of their emotional state (Nguyen et al., 2018). These sensory deprivation practices may be common when meditating; however, it is more likely that individuals will seek out or create places which are imbued with pleasant sensory qualities and pursue activities which make them feel relaxed, happy, or inspired; positioning solitude not just as an escape, but as an opportunity to take control of one's environment, express oneself, and be creative (Altman, 1975; Long \& Averill, 2003).

\section{Privacy, autonomy, and freedom}

When an individual is feeling overwhelmed by social and environmental stressors, there are two key advantages that solitude has to offer. The first is privacy. Public law professor Westin (1967, p. 7) defined privacy as, "The claim of individuals or groups to determine for themselves when, how, and to what extent information about them is communicated to others, and from others to them". This concern over the intersubjective exchange of information highlights a desire for some level of choice or control over oneself and one's environment, including the company that one keeps and the way in which one is being represented or perceived.

Privacy was particularly important for the girls and women as it offered an escape from the usual constraints and expectations that required individuals to act a certain way in public. 
In particular, participants appreciated having privacy to cry and relieve feelings of anger or frustration. As Sui Sung mentioned, she would often pray and cry at home and in her garden. Having these private places was important as, with the exception of young children, females who openly cry in New Zealand may be labelled as irrational or attention-seeking and males are generally considered weak (Suckling, 2016). These negative perceptions of vulnerability play into a wider cultural proclivity for strength and resilience and a stoic 'she'll be right' care-free New Zealand identity (McCool, 2017).

For former refugees, emotional outbursts also run the risk of reinforcing negative stereotypes of being vulnerable or traumatised and a 'burden' on society (Beaglehole, 2013; Ford, 2012). Recent research on the concept of resilience has formed a powerful counter-narrative to the disempowering and misleading notion that all refugees are traumatised (Lenette et al., 2013; Munt, 2012); however, it also raises expectations of strength that may be difficult to maintain, and overlooks the cathartic value of crying in reducing everyday stress and tension. Burger (1995, p. 88) claimed that,

Although time spent with others typically is pleasant, concern for what others think and the constant monitoring of others' reactions can become emotionally draining. Even a short period of time by oneself before returning to social interactions can serve as a kind of emotional renewal.

Whilst social campaigns are beginning to challenge attitudes around how New Zealanders publicly deal with emotion (Health Promotion Agency, 2020; The Key to Life Charitable Trust, 2020), opting to have personal time and space away from others remains important for individuals to let down their guard and relieve stress free from social stigma. Psychologists Nguyen, Ryan, and Deci (2018, p. 104) claimed that, "People can use solitude, or other variations on being alone, to regulate their affective states, becoming quiet after excitement, calm after an angry episode, or centered and peaceful when desired".

The second key advantage of solitude is the opportunity to control one's external environment. In the absence of other people, one has less need to negotiate the use of space (Altman, 1975; although non-human entities may still pose some restrictions), and can create an environment tailored to their specific needs and multisensory preferences - 
defined as the types of senses that make them feel relaxed, comfortable, or alert (Champagne, 2004). Long and Averill (2003, p. 38) claimed that,

When in a situation of reduced social stimulation, the person whose characteristics facilitate feelings of comfort and control over his or her particular surroundings is more likely to find solitude rewarding than is the person prone to feel at the mercy of that specific environment.

Thus, the girls and women could get away from the strange new smells, tastes, and textures of New Zealand cuisine and cook their favourite dishes. Or, like Mu Pli, they could play their favourite music and sing and dance. Music psychologist MacDonald (2013, p. 7) claimed that,

Music ... acts as a stimulus that is distracting. When we are listening to our favourite music we are not attending to the noxious stimulus or at least attending to it with less intensity. Listening to our favourite music is also emotionally engaging so this emotional engagement draws us away from the noxious stimulus.

Geographers Kearns and Andrews (2010, p. 103) also said that music, "Can help change both us and the places we frequent. It can help create a mood or atmosphere in a place, where it represents an auditory backdrop to our daily lives”. Because of this transformative potential, one of the resettlement practitioners added, "Music is probably something that is under-utilised as a therapeutic modality. Singing is a fantastic one for anxiety as well”.

Being able to control their environment also meant that the girls and women could maintain their cultural identities in ways that they felt unable to in public. Elizabeth said,

In my village [in Chin State] ... my family going to celebrate Christmas everyone come to our house to help each other clean or decorating the house, helping for food at home, and the young people singing a Christmas song. But if we do here, we worry about neighbours!

Concern over neighbour's reactions to cultural food and music was significant considering the negative backlash that participants had sometimes received in Nelson for looking or sounding different. However, in the comfort of their own homes, they felt less pressure to behave a certain way and expressed a feeling of freedom. When talking about cooking in her kitchen, Mai Aye Aye said, "I feel free to be myself". 
Freedom can also inspire creativity. Long and Averill (2003, p. 26) claimed that, "The daily environment of most people is highly structured. If environmental influences are radically reduced or altered, mental structures, too, may be disrupted, thus allowing thought to become more fluid and differently organized". They also suggested that being alone enables individuals to try on alternative realities, which can potentially contribute to processes of creative self-development and transformation.

For former refugees who have spent time in overpopulated refugee camps and highly structured and oppressive detention centers, or who live in crowded homes and are used to being intruded upon, or intruding into the space of others, these solitary creative spaces can be significant. During the time he spent in Australia's controversial refugee detention centre on Manus Island, Iranian asylum seeker Behrouz Boochani (2018, p. 127) wrote,

For me, isolation and silence are the greatest gifts I could ask for. Faced with prisoners who reach out to their mates by yapping, yelling pointlessly and laughing loudly, I long to create, to isolate myself and create that which is poetic and visionary.

Yet, Boochani (p. 132) also highlighted the need for ongoing social interaction and support. He claimed that, "Probably the life most worth living is a solitary one. A quiet life, a lively existence, and a glorious one. But what a bitter life that is".

\section{Balancing alone-time and social participation}

Whilst solitude does come with a risk of loneliness, one is very rarely (if ever) completely alone. People have sociable memories and thoughts (Koch, 1994), and Long and Averill (2003 p. 28) claimed that, "Loneliness can be kept at bay as long as we are aware in some way that we have meaningful connections to other people". Assuming one hasn't deprived oneself of, or been deprived of, surrounding stimuli, then it is also likely that an individual will have some form of contact with non-human entities. The relationships that the girls and women had developed with the local flora and fauna in Nelson were significant in preventing feelings of loneliness; and as Christians, they also held the belief that they were always in the presence of God. Ngai Ciang Sung's experience of being alone on a mountain, but feeling connected to 'everything', further demonstrates an ontological awareness of being part of something intrinsically greater than oneself - of "co-belonging with the flesh of the world" (Hawkins \& Straughan, 2014, p. 132). 
In her comments above, $\mathrm{Mu} \mathrm{Pli}$ also talked about looking through social media and watching YouTube when she was alone in her room, and in the previous chapter participants spoke about their frequent use of social media to stay in touch with family overseas. Recent research on mobile communication technologies and transnational homemaking also highlights the value of social media in enabling individuals to feel connected to distant others (Marlowe, 2019; Ozkul, 2013). These moments of virtually beingtogether, yet still physically being alone, bring new, relatively unexplored dimensions to the concept of solitude, and require a much more in-depth look at how technology might keep loneliness at bay.

Yet, these spiritual, more-than-human, or virtual interactions are not the same as social contact, and participants always planned to re-engage with the Nelson community and city when they had the time and energy. This was evident in the womens' comments, as they spent their alone-time preparing for future social engagements. Sui Sung said that she liked to go "walking, and you know, I'm very like collect mussels! ... Too many! I get too many [mussels]. Bring them back for my friends and say, 'who would like?' Yes! Oh! I'm very happy". Even those individuals who had previously expressed a strong preference for solitude desired some degree of meaningful social interaction in their daily lives. Mu Pli said, "I do sometimes wish that ... I mean I love meeting people ... so I would appreciate it if people from other cultures want to be friends with me. I'm totally up for it'.

Continuing to strengthen local social connections during resettlement is particularly important as, if an individual does not have a supportive community or accessible city to return to, then what may have begun as a choice to spend time alone can quickly decline into a feeling of social isolation. Burger (1995, p. 96) claimed that,

It is possible for people to enjoy time alone and yet also feel that they do not have the kind of social relationships they desire. In fact, because people with a high preference for solitude often spend time by themselves they may be less successful at developing the kind of social relationships that prevent loneliness.

Therefore, a balanced approach to refugee wellbeing is needed, whereby individuals can engage in social life and then retreat, reflect and re-energise with the reassurance that even in their absence they are part of something meaningful, to which they can always return. 
Whilst international resettlement research continues to prioritise social participation and integration, a more balanced approach is beginning to be explored through concepts of 'labouring and resting', which were teased out during the 2019 UNESCO Rila Spring School in Glasgow, Scotland (University of Glasgow, 2019), and through Phipps' (2019b) De Carle lecture series in Dunedin, New Zealand. The concept of solitude was not explicit in these discussions; however, the idea of resting is synonymous with much of the existing literature on therapeutic landscapes and will be significant in future framings of solitude as a form of emotional renewal.

Indigenous models of wellbeing will also be central to a more balanced approach. The Māori models of Te Whare Tapa Whā (Durie, 1985), and Te Wheke (Pere, 1997) in New Zealand, and the 'medicine wheel' of the Anishinabek people in North America (Wilson, 2003) have long recognised that wellbeing is comprised of physical, emotional, mental, social, and spiritual balance, and that when one element is favoured or neglected, an individual's overall state of health suffers. These models also do not explicitly mention solitude or necessarily focus on taking 'time-out', but provide the wider wellbeing framework to which solitary experiences can contribute.

\section{6:4 Key learnings}

Whilst homes offered places of sanctuary for participants to rest and feel safe in Nelson, the girls and womens' spatial and spiritual encounters with beautiful views, diverse wildlife, and unique multi-textured terrain were also significant in evoking positive and comforting memories, and prompting new feelings of curiosity, enchantment, and connection. The ability to feel safe, enchanted, and connected through the senses is particularly important in refugee resettlement, as these experiences provide a possible means through which individuals can begin to form an emotional bond with a place; even when they have only recently arrived and are situated in an unfamiliar environment.

However, one of the main points that I want to draw out from this chapter is that places are not inherently healthy or therapeutic (Conradson, 2005), and what is beneficial to one individual may not be so for another. How an individual feels in a place depends on a wide 
variety of factors, including their unique memories, personal preferences, age, gender, ethnicity, class, ease of mobility, and changing physiology. Therefore, whilst, physical, social, virtual, and spiritual environments each have the potential to make former refugees feel well or attached to their new city, exactly how this potential is realised will differ for each individual (Collins \& Kearnes, 2007; Milligan \& Bingley, 2007).

Another point which I wish to elaborate on is the ambivalence and precarity of emotional people-place relationships, or the ability to fluctuate between positive and negative emotions as stimuli changes. This precarity becomes particularly salient in light of the discussion on solitude. Whilst therapeutic-feeling places often encompassed nontherapeutic elements, solitude enabled participants a greater degree of control over space to maximise their level of comfort. In this manner, solitude offers an opportunity to create a more customised therapeutic experience, allowing one to maximise a place's therapeutic potential and further enhance one's sense of wellbeing. Time alone for rest and emotional renewal is particularly important considering the difficult work and feelings of fatigue that participants faced in navigating unfamiliar places and languages and dealing with 'scary' racist and antisocial behaviour.

In advocating for a focus on therapeutic-feeling places and solitude, I hope to contribute to a more balanced approach to refugee resettlement. This balance not only means juggling the work of resettlement with time out to rest, or juggling social time and time alone, but also balancing these needs with other physical, mental, emotional and spiritual needs. In terms of researching holistic frameworks of resettlement, geographers and other social scientists also need greater balance in the way that they theorise lived experience. This means moving beyond the predominantly visual frameworks which have dominated work on 'green' and 'blue' therapeutic landscapes (Bell et al., 2018; Foley \& Kistemann, 2015), to focus on the multisensory and multi-textured experiences (Brown, 2016; Gorman, 2017) that make places feel scary, or that leave one feeling enchanted and curious about their place in the world. 


\section{Chapter 7: Time, Familiarity and a Sense of Belonging}

Despite the girls and women feeling instantly attracted to places which evoked feelings of homeliness, safety, and happiness, these in-the-moment experiences did not necessarily generate the same deep sense of familiarity, pride, and belonging that ongoing sociospatial engagement can foster (Hay, 1998; Lewicka, 2011; Porteous, 1976). In psychologist Lewicka's (2011) interdisciplinary literature review, residence time (the length of time that an individual has resided in a geographical locale) consistently rates as the most positive place-attachment indicator. Thus, former refugees who have been resettled for a significant period of time are likely to demonstrate stronger feelings of place-attachment than those who have only recently arrived. To understand how emotional sociospatial relationships are strengthened over time, this chapter explores participants' accumulating multisensory experiences over the days, weeks, months, and years of resettlement. I also discuss how ownership of, investment in, and nurture for a place over time can entangle people and places in mutually co-dependent relationships, whereby each becomes defined by and intimately connected to the other (Bawaka Country et al., 2016; Wright, 2015).

\section{7:1 Becoming familiar with new places and rhythms}

\section{Connecting to 'places of need'}

One of the reasons why time may enhance feelings of place-attachment is that it enables the opportunity for individuals to become familiar with their surroundings through exposure to new stimuli (Lewicka, 2011; Zajonc, 1968). As human geographer Seamon (1980) claimed, repetitive exposure to one's environment can lead to ingrained bodily habits and comforting place-based routines. In this manner, familiarity is felt in the way that one's body automatically knows when to turn or duck, or jump over a particular crack in the pavement. However, familiarity is also a cognitive process of acquiring environmental and social knowledge. Social psychiatrist Fullilove (1997, p. 1518), claimed that, "This knowledge accumulates through a complex, trial-and-error process of acquiring and processing sensory information to create cognitive models of the surrounding world". 
Anthropologist Hage (1997, p. 103) also talked about familiarity as 'maximal spatial knowledge': "Knowing what everything is for and when it ought to be used ... knowing almost unthinkingly where one is, and where one needs to go for specific purposes and how to get there". He claimed that this knowing also implies spatial control and fosters a sense of security. Geographers Mathisen and Cele (2020, p. 12) claimed that, "Gradually getting to know an environment, then, is connected to material aspects of place such as finding one's way and knowing where one is".

During the first six months of resettlement, local organisations and volunteers played an active role in orientating newcomers in Nelson and enhancing these processes of familiarity. A practitioner from the Red Cross Pathways to Resettlement Programme said,

The people that engage with them [former refugees] initially, that is our staff and volunteers, and other agencies and people from other agencies, that's where their connection to place happens... And that's everything from taking them to the Igeneral practitioner], taking them to the shop, having a picnic on the beach. You know, showing them how to cycle, where the cycle routes are. It's all of those hundred-andone things that our volunteers do to facilitate with the connecting of that wider place of Nelson. You could imagine if there wasn't that, some people would just close into their home and their immediate family.

In identifying the types of places to which newcomers were being introduced, one of the practitioners claimed that, "There are probably places of need, and places of pleasure, and places of everyday happenstance". Subsequent interviews tended to support this categorisation, and practitioners agreed that helping newcomers to become familiar with places of need - including homes, schools, workplaces, hospitals, social services, and shops - was a top priority.

When it came to connecting to her new home, Elizabeth said that her Red Cross volunteers helped her to learn about, "All the stuff, the pot, the rice, and the fridge. I never used a fridge before! ... [I] didn't have fridge and washing machine and stove”. These foreign objects initially held little meaning for participants; however, as they became familiar with the way that such material objects looked, felt, and operated they could appreciate their value. Sui Tin Men claimed that after learning how to use her oven it was much easier than 
lighting a fire back home. She said, "Fire is very hard, electric easy. Easy to clean". Meanwhile, Zai Zai had discovered a unique textural benefit to using her electric blender. She claimed that whilst vegetable soup in Myanmar is served with cut-up vegetables and can be difficult to chew, New Zealand soups are often blended which makes it easier for parents to share this dish with their young children. An element of enchantment with these objects potentially sped-up processes of familiarity as the women were engaged and eager to learn (Phillips et al., 2015).

Other initiatives that helped former refugees to become familiar with local systems and ways of life were pre-election meetings to discuss how to vote (a right that comes with permanent residency and citizenship), and parenting courses which helped newcomers to feel that they were giving their children the best possible start in their new country. Practitioners also highlighted the value of educating newcomers on health and safety procedures. One practitioner said, "What I've heard from Burmese people is around fires. When they are living in places in really cramped quarters, and in areas where houses are built really close together ... the impact of fire is really traumatic”. This point was reiterated by another practitioner who had previously worked at the Nelson Marlborough Institute of Technology (NMIT) and said, "When we had fire practices we had to make sure they [former refugees] knew before it was going to happen and let them know it was just a practice".

Meanwhile, a practitioner from the Victory Community Centre said,

One of the other things which we have done here is antenatal classes for those [for] who[m] English is their second language. With an interpreter ... one of the midwives said she had noticed that some of the women were having worse experiences here than they had in the refugee camps because they didn't understand, and it hadn't been explained, how the hospital system works. So, running those antenatal classes has been really important.

The fact that some women felt more comfortable giving birth in a refugee camp than in a modern Nelson hospital shows that a lack of understanding around local birthing practices significantly impacted their ability to feel in-control of the intimate situation at hand. Introducing the antenatal classes made a difference to how these women felt being in the 
hospital and removed some of the anxiety that came with trying to navigate unfamiliar spaces and practices during an already stressful time.

Together, these practical and informative meetings and courses and engagements with the city played a significant role in place-attachment as individuals gained confidence in navigating places of need and participating in social and political activities. Such experiences can enhance feelings of autonomy and belonging, and foster a sense of attachment through positive emotional and spatial associations (Mathisen \& Cele, 2020; Scannell \& Gifford, 2010; Tuan, 1974).

\section{'Places of pleasure and everyday happenstance'}

Practitioners and volunteers also helped former refugees to become familiar with 'places of pleasure' (commonly referred to in geography as leisure or recreational spaces). These places can be distinguished from places of need in that they are not crucial for human survival, at least not in the immediate ways that housing and hospitals are. However, as discussed in Chapters Five and Six, they can be significant in terms of everyday wellbeing. One of the practitioners said that, "English Language Partners might take them [former refugees] out to Cable Bay or a trip to Rabbit Island". The girls and women also spoke about exploring places like the Queens Gardens and the Maitai River with friends and family. Other recreational places included city venues such as local cafes, restaurants, the zoo, and the cinema. Little Ruth said that she enjoyed going to the movies with her local volunteer/friend, and claimed that she had recently watched a film called 'The Kid Who Would be King', which gave her courage to start her new school (she was due to start college at the time that I interviewed her).

These recreational places are also 'places of everyday happenstance', where chance encounters with strangers or unfamiliar objects and entities are likely to occur (Seamon, 1980; Stevens, 2007). Chance encounters may also happen in private places; however, one generally knows who (or what) will be in their house or office at any given time, and the regular movements that these familiar individuals and objects will make during the day. Visiting the cafe or the beach on the other hand offers greater potential to cross paths with new people or non-human entities (such as strange foods, plants, or animals). 
These spontaneous free-flowing places could also constitute what geographer Massey (2005, p. 141) refered to as sites of 'throwntogetherness', or the "coming together of the previously unrelated". Unexpectedly being thrown-together with others has the potential to evoke curiosity and enchantment (Geoghegan \& Woodyer, 2014; Wilson, 2017); however, it can also evoke fear. Elizabeth said,

Walking around, like for example, Maitai Dam, to get fresh air, but you know is ... sometimes you can meet bad people so I am scared to go around there ... Accident happen everywhere you know and you don't see when it is going to come. So, I go around there and it makes me feel unsafe.

It is difficult to prepare oneself for a chance encounter. However, through becoming familiar with the physical characteristics of places and who tends to frequent them, individuals may be able to build up confidence that, if an 'accident' did occur, they would know where to go or what to do to minimise harm and regain a sense of safety. Thus, even the unknown can be less scary in a familiar-feeling environment.

Mobility is integral to these processes of familiarisation, as movement enables individuals to actively expand their spatial experience (Gallagher \& Zahavi, 2008; Seamon, 1980), and "acquire our ever growing sense of what our world is like" (Johnson, 2007, p. 21). Philosophers Gallagher and Zahavi (2008, p. 98) have claimed that, "What we see and hear and touch and taste and smell (or avoid smelling) is shaped by what we do and what we are capable of doing - our pragmatic possibilities and the sensorimotor capacities of our bodies". Because bodies and sensorimotor capacities (the potential to move) differ for each individual, exposure to new stimuli and the opportunity to become familiar with diverse places will also differ, leading to unique place-based experiences. One of the practitioners said,

I know that English Language Partners take groups of [former refugees] for a walk around town, an orientation, but I don't believe they do it on their own ... I do think that many of them are well used to walking the distance. It's not that they can't walk the distance. Although there was quite an older group of refugees I saw one day ... 
For elderly people who have trouble moving, the relatively short trip into the city may be tiring. Several of the women had also been pregnant while they lived in Nelson and were now caring for small children, circumstances which also hindered mobility. Jenavi said,

You know, I have a lot of places [to go], but I have children. The children are still young so I don't do anything anymore. Some people say [they go to] Kaiteriteri, or something like that, but I never go. Just stay home and look after children. So, I dunno [this] place, just only I heard ... I would like to go.

As previously mentioned, prevalent female health issues such as anemia could lead to fatigue and also make 'walking the distance' problematic for some women, and mobility could also be curtailed by a lack of access to transport (Bose 2014; Kale \& Kindon, 2020; Martin 2008; Swe 2013; Urry 2012). One practitioner said,

Learning to drive is a huge problem. The Red Cross are putting through one person per family on the Open Road Programme, but often it's a man and the wife has to get the groceries and pick up the [children from] school ... But also, he [the husband] can't teach someone else to drive for another two years after that. So, it's six months to learn, six months to get your full license, and then two years after that! So, it is three years before he can teach [his wife]. Most of the Colombian men have their license already, but they can't teach their wives.

She added that accessing many of the beaches and wilderness areas could also be difficult as, "There is no bus service out there".

These physiological and social barriers are important to keep in mind as they suggest that some individuals may take longer to get to know a place than others, or that they may be restricted in the types of places (and people or non-human entities) with whom they can interact. These restrictions were also highlighted in sociologist Gustafson (2009) and geographer Westin's (2015) mobility studies, which demonstrated that women felt more attached to local residential areas than men, who were more mobile and had the opportunity to build attachments to more distant and diverse places.

These familiar places begin to build up meaning over time, as, whilst experiences may be fleeting, they draw in 'multiple temporalities' (Wilson, 2017). In this manner, an individual is not only experiencing the present moment in a place, but also remembering the last time 
they visited, or the time before that. They may also be thinking forward to future visits. In Mathisen and Cele's (2020, p. 12) research with young former refugees in Norway, one of their participants described regular walks through the neighbourhood with her father. The authors claimed that, "The walks they took together became loaded with positive meaning that was transferred to the places they visited. The physical environment became transformed from locations into lived places, as they were experienced with emotion". Such layering of emotional experience over time can sometimes lead to attachments with seemingly mundane objects. As one resettlement practitioner said, "We all get attached to the weirdest things, like the fish and chip shop you walk past every day, do you know what I mean? To everybody else it means nothing but to somebody it means the world. It's that smell, it's that colour". However, these places also changed over time, meaning that familiarity with place was an ongoing process extending far beyond the initial few months of resettlement.

\section{Navigating dynamic environments}

During their initial sociospatial orientations, practitioners helped newcomers to navigate the changing characteristics of place by orientating them with Nelson's unique seasonal cycles. These temporal orientations were important in assessing risks and maintaining a sense of comfort, safety, and good health. As mentioned in section 6:2, maintaining warm dry homes in the New Zealand winter is a nationwide problem, and can be particularly problematic for families living in lower-socioeconomic neighbourhoods like Toi Toi (Nelson Marlborough Health, 2016). One practitioner said,

[Nelson's] cold houses and the areas in which cheaper housing is available means that they [former refugees] are living in areas in the shade. They can be colder older houses - that will all impact on their sense of wellbeing. I know what it's like when it's cold and your house is in the shade and how it feels.

Whilst the Red Cross and Victory Community Centre offer clothing and heaters to families in need, damp mouldy houses have been linked to asthma, respiratory infections, the exacerbation of chronic conditions, and poor mental health (Nelson Marlborough Health, 2016). These environmental conditions and bodily ailments could potentially hinder processes of place-attachment and belonging by evoking feelings of insecurity for those 
who feel the cold, smell the dampness, and have trouble breathing and feeling well in their homes.

Not being used to this type of housing and the New Zealand climate, former refugees often lack the tips and tricks that local New Zealanders have developed over the years to minimise mould and dampness (such as wiping down walls, using dehumidifiers, and blocking draughts under doors). Another practitioner said, "Their houses are cold ... they are not necessarily prepared for that with clothing, and don't know about warming and ventilating houses, you know, creating a healthy home”. Iang Chin Sung supported this claim, saying, "When we arrived in Nelson it was May and really cold. My second son was only six months old and it was cold in the house and outside. We didn't bring any warm clothes with us, but people helped". The sharing of local knowledge by long-term residents is helpful in these situations, yet understanding the best way to warm one's unique dwelling to their individual liking also takes time and what psychologist Fullilove (1997) described as a process of 'trial-and-error'.

Poor ventilation can also be a problem in the Nelson summer. Ngai Ciang Sung said, "I don't like to be inside in summer because it is too hot. I feel dizzy ... I like camping because fresh air". Ruth added that she only spent time in her kitchen in the morning or evening during the summer as it was uncomfortable to cook in the midday heat. Several of the women also mentioned that they changed their gardening practices to avoid the risk of heatstroke and sunburn. Whilst sunburn is a global phenomenon, a depletion of ozone (an atmospheric gas which absorbs harmful ultraviolet radiation from the sun) has contributed to New Zealand's melanoma cancer rate being two to three times higher than that of other Western countries (McKenzie et al., 1999; Nartey \& Sneyd, 2018). Thus, acts of self-care and protection that may be adequate in other countries are not necessarily appropriate in New Zealand and adapting to new seasonal behaviours may take time to get used to.

These experiences reiterate the idea that place is not simply an object of affection waiting to be perceived and adapted or loved by people, it is an autonomous entity in its own right, capable of impacting human bodies, emotions, thoughts and behaviours in various ways (Ash \& Simpson, 2016; Larsen \& Johnson, 2016; McCormack, 2017). Learning about these unique geographic cycles and the risks that they bring is a significant part of 
maintaining a healthy relationship with place, and feeling as though one can mutually coexist with their environment without one's skin blistering in the sun or airways contracting in the cold.

However, knowing the seasons was not only conducive to moderating risk, but also maximising enjoyment. In their paintings of ANZAC Park, Mu Pli and Mai Aye Aye both symbolically represented the vibrant yellow Marigold flowers which are planted in late spring by the Nelson City Council - an enchanting phenomenon that is lost during the winter (see Figures 6:2 and 6:4). Mu Pli had mentioned that she would go to the park specifically to see these 'intriguing' flowers and Mai Aye Aye explicitly stated that she enjoyed visiting in 'spring-time' because the flowers were 'pretty'.

I didn't become fully attuned to the significance of these symbols and comments until one day when I set off on one of my regular reflexive walks up Ngawhatu several hours earlier than usual. Knowing Ngawhatu to be a peaceful place from my many evening visits (as described in section 4:2), I was surprised to find myself feeling annoyed at the afternoon heat and wind and the unharmonious sound of unruly children, dogs, and scooters. Analysing these feelings as I walked, I realised that,

I was not only drawn to the spatial terrain, but a particular light that was quintessential of a Nelson summer sunset. It was that specific time of day in Ngawhatu when the dizzying temperature had dropped a few degrees, the wind had decreased to a gentle whisper, and the residents had disappeared inside their houses and left the streets quiet and empty, creating an alluring sense of stillness and calmness in the valley. Being there in the more-chaotic afternoon rhythms ruined the almost surreal magic that I'd come to expect as the day faded into dusk, and left me feeling somewhat disappointed in this place by which I had previously been enamoured (Field notes, 01/02/2019).

Ethnomusicologist Hahn (2006) claimed that such disorientating or surprising sensory experiences can jolt researchers into new understandings or ways of knowing. In this case, experiencing the multiple and diverse rhythms of place for myself was an important step forward in my research, as it highlighted the need for a more nuanced temporal understanding of place-attachment, whereby feelings toward place are understood to not 
only strengthen (or diminish) over time, but to constantly ebb and flow along with the shifts in social routines and seasonal cycles (Lefebvre, 2004; Potts, 2015; Seamon, 1980).

\section{7:2 Finding 'a comfortable place with each other'}

\section{Learning to be Kiwi}

Time also enabled greater familiarity and connections with local residents and 'Kiwi' (New Zealand) culture. As mentioned in the previous chapter, one of the biggest barriers to building cross-cultural relationships was language. Whilst several of the women had said that listening to and speaking a foreign language could be scary, Jenavi claimed that months of hard work attending English classes and learning the pitches, tones, and nuances of the English language had enabled her to communicate with her neighbours when she passed them on the street, and had been crucial to making her feel more confident and athome when navigating her way through her neighbourhood and city. Geographer Antonsich (2010, p. 648) claimed that shared language can, "Evoke a sense of community, the 'warm' sensation to be among people who not only understand what you say, but also what you mean". This understanding plays an important role in constructing a shared sense of reality (Echterhoff et al., 2009), and feelings of intimacy (hooks, 2009), and belonging (Antonsich, 2010).

Becoming familiar with New Zealand food over time also improved participants' gustatory and olfactory experiences. Jenavi said, "When I arrive the first time to here, no, I don't like very much to smell and everything. But now I like. I like everything Kiwi food”. Elizabeth added that when she first arrived in New Zealand, "We stayed there in Mangere camp for six weeks and for me the food was a nightmare. And after that we came to Nelson and can cook our food. We cook ourselves about a week and then I miss the Auckland food again!" These adaptations to Kiwi culture were important in helping the girls and women to feel like they were part of New Zealand society. However, such a focus on becoming Kiwi could also make participants feel pressured to assimilate and replace their own cultural traditions and ways of life with the dominant behaviours of the locals, effectively becoming one of 'them' (Blunt \& Dowling, 2006; McNeill, 1994). 
As in other resettlement studies (Arev, 2018; Beaglehole, 1990; Lewis, 2015; Rishbeth et al., 2019), processes of assimilation were visible through participants' choices of clothing. Almost all of the girls and women had described the traditional sarongs worn by males and females in Myanmar, and showed me beautiful woven fabrics or photos of these garments during their interviews. However, in Nelson they had bought Kiwi clothes to blend in with the locals. Specific traditional colours from Myanmar were also avoided to fit perceived New Zealand gender norms. Ngai Ciang Sung said, "New Zealand is different because men, boy you know, they not allowed to wear pink. But in Myanmar is okay, everyone has to ... [it] doesn't matter".

Concerns over clothing were also expressed by refugees resettling in New Zealand in the 1940s, 50s and 60s. Historian Beaglehole (1990, p. 56-57) mentioned the embarrassment that former refugee children felt when they were dressed differently to their schoolmates. Whilst she acknowledged that long-term residents may also be considered misfits due to their clothing, she said, "The difference becomes apparent, however, when one considers that the taunts suffered by some refugee children about their appearance were repeated in many other areas of their lives". In geographers Rishbeth et al., (2019, p. 130) study with asylum seekers and refugees in the UK and Germany, the authors also claimed that, "Difference in dress can lead to a greater (and problematic) feeling of hyper-visibility".

Some individuals may be happy to assimilate into (or partly assimilate into) a new culture, particularly if they wish to break ties with a painful or oppressive past or do not harbour strong attachments to their former way of life. A participant in social researchers Elliot and Yusuf's (2014, p. 109) New Zealand resettlement study claimed, "We need to move on or move away from the back-home politics and just focus on here. Simply to say, 'Here's our country, here's our home' - how can we be part of this?" Adopting the norms of another culture may also be empowering or liberating. In research with female former Eritrean refugees in Tel Aviv, Israel, anthropologist Arev (2018, p. 317) claimed that,

The right to choose their clothing - a right first emerging when they encountered Israeli society-becomes central for the refugee women. The comfort of the clothes is tied, first and foremost, to the absence of uniqueness. Their incidental and informal style allows the refugees to move away from the dimension of otherness in their identity and is thus an expression of an all-embracing Westernness. This shift is a 
political act that aims to expand possibilities and dissolve the old cultural gender order. The women will no longer be wrapped in traditional clothes that serve the community; instead, they become active players who carry on their body the values of independence and equality.

Age may also play a role in willingness to assimilate. Little Ruth said, "I don't miss the culture. I don't really care about that. But I know my aunty does". Those who are older, and who have spent longer familiarising themselves with unique cultural habits and traditions, are often more reluctant (or unable) to give up the homely practices that contribute to a sense of self identity and make them feel well and happy. Having spent less time being socialised into a particular culture, children may be less habitual and more open to change and often adopt the norms of the local society faster than their parents (Collie et al., 2010; Marlowe et al., 2014).

These intergenerational differences could sometimes cause tensions within families, and the women often felt pressured to assimilate through their children asking for Kiwi food to take to school (a point also noted in Longhurst et al.,'s 2009 research with migrant women in Hamilton). Jenavi said, "I can't cook ... My children like Kiwi food, and my son say mum, make food like that, and I say I can't". To appease their children, a local kindergarten teacher (who initially helped me to recruit research participants) claimed that former refugee parents purchased lunch packs from the dairy (corner store) down the road. These packs consist of low-nutritional, sugary snacks and can have negative long-term health implications and affect children's concentration and ability to learn. Unfortunately refugee-background parents were often unaware of these risks due to being unfamiliar with processed products and unable to read the dietary information printed in English. Thus, they were unwittingly trading the benefits of healthy home-cooked food for their children to feel accepted by their peers.

To minimise the pressure on former refugees to give up meaningful practices and traditions, yet still enable them to engage in local ways of life, resettlement literature has shifted away from theories of assimilation toward social integration. In this manner, "The challenge is best met not by making "them", like "us", but rather by creating a new, more capacious sense of "we"' (Putnam, 2007, p. 163-164). Thus, there is a greater emphasis on 
cross-cultural recognition and the sharing of knowledge and fostering of mutual respect for different people and ways of living (Askins, 2016; Kale et al., 2019; Strang \& Ager, 2010). These practices of social integration are also central to Nelson resettlement praxis, as evident through the wide range of cross-cultural initiatives discussed below.

\section{Fostering mutual recognition and respect}

To normalise diverse cultural sights and smells and tastes and accommodate an increasingly multicultural population, Nelson resettlement organisations host multicultural events in the city. A practitioner from the Nelson Multicultural Council stated that,

Events are probably the thing that we do the best, and most consistently is Race Unity Day and another food event - Tasman's Asian Night Food Fair ... We also do intercultural awareness courses. And they are attended more by employers, by community organisations, school teachers and that sort of thing, not so much by the refugees or migrants themselves. So, it's educating the community to try to generate a better understanding.

Cultural events such as Matariki (New Zealand's indigenous Māori New Year) are also directed at bringing diverse communities together to enhance cross-cultural understanding and build a shared sense of identity, and refugee-background communities often invite the wider community to their own traditional celebrations. The practitioner from the Chin community said, "Every year we have a cultural diversity day. And Chin students present their own dance, and sharing food, you know, different kinds of food. And parents also come and help us, and we have a show - a play for our custom”.

These events resonate with cultural theorists Christiansen, Galal, and Hvenegaard-Lassen's (2017, p. 601) ideas of 'organised cultural encounters', which aim to bring diverse individuals together "in close bodily proximity in order for them to experience 'the other' as a real physical presence beyond stereotypical representations from afar". The authors claimed that,

Organised cultural encounters are - to use a theatre metaphor - scripted events: time, place, roles and interactions are more or less strictly prescribed and regulated in advance of the encounter. The scripts are also tied to the particular social arena with which the encounter is associated. That is, organised cultural encounters take place 
within already established professional or institutional contexts, and are thus shaped in important ways by the existing norms, discourses, roles and hierarchies that govern these arenas.

Whilst the 'scripting' of such events may appear to lack cultural context and authenticity, there can be benefits to hosting these types of events. As one of the practitioners mentioned, there was an opportunity for cross-cultural education and understanding, which could take place in a safe controlled setting (Christiansen et al., 2017). Psychologists Brown, Perkins, and Brown (2003, p. 261) also claimed that, "Neighbourhood events and interactions that promote cohesion also allow residents to know and value the homes, sidewalks, parks, and shops that constitute the physical fabric of the neighbourhood, potentially enhancing place attachment".

As mentioned by one of the resettlement practitioners, cultural events also enabled former refugees to feel as though they had something valuable to contribute to the community. Reflecting on his experience in Tauranga (a city in New Zealand's North Island), he said, One of the things that made them [former refugees] most accepted into the community in Tauranga when I was living there was a cultural day. It was a festival where all the different Indigenous groups would come together in a park ... and they would have all these food stands. People loved to go there, and they would dress up, and there was beautiful dancing and costumes and things. It was just a fantastic way of them giving value to their community and people were thinking, actually, these people have something to offer, this is beautiful, this is fantastic, and normalising those people and things. So, I think those sort of community events are a wonderful way to not only make people more understanding and welcoming, but also because they are getting the applause they feel as though they are being accepted into the community as well.

In this manner, there was a sense of recognition that diverse individuals existed within New Zealand and could add value to society. Recognising diversity is important in enabling individuals to feel as though they belong for who they are, not just for how Kiwi they appear. As one of the participants in my Masters' research said, "I feel like I belong when my culture and who I am is appreciated" (Kale et al., 2019, p. 12).

However, as feminist theorist Ahmed (2000) and geographer Wilson (2017) argued, difference emerges from social encounters. Thus, events which promote diversity could 
also potentially remind individuals of how 'foreign' they are and exacerbate feelings of not belonging. Mu Pli said, "I don't actually make friends with people from [Nelson] ... I think I feel different". These cultural performances also risk promoting a superficial type of multiculturalism or touristic 'cosmo-multiculturalism' (Hage, 1997), whereby ethnicity or diversity is viewed as an object of consumption that can be enjoyed without having to get too close to or interact with ethnic or cultural 'others'.

To shift the focus away from ethnocultural differentiation, social science scholars have focused on the concept of 'conviviality'. Cultural theorist Gilroy (2006, p. 40) claimed that conviviality is a social pattern in which communities with diverse ethnocultural backgrounds live in close proximity without any (significant) issues or emphasis on skin colour. He said,

In this convivial culture, racial and ethnic differences have been rendered unremarkable ... they have been able to become 'ordinary'. Instead of adding to the premium of race as political ontology and economic fate, people discover that the things which really divide them are much more profound: taste, lifestyle, leisure preferences. By making racial differences appear ordinary and banal, even boring, convivial interaction ... has disseminated everyday virtues that enrich our cities, drive our cultural industries and enhance our struggling democracy so that it resists pressure to operate in segregated and colour-coded forms.

Such conviviality paves the way for the recognition of diverse others as similar in many ways. Multicultural theorist Noble (2009, p. 46) claimed that there is a need to distinguish between, "Recognition as (I am this, you are other), and recognition with, the mutual, collective fashioning which comes out of shared practice". Geographer Wright (2015) talked about this recognition-with as 'feeling-in-common' with others, or a type of mutual solidarity. Building on these ideas, geographer Askins (2016) discussed the importance of mutual recognition in moving beyond the labelling or categorising of others to viewing them as someone relatable - someone with the potential to be a friend. Such befriending is important as cultural theorist Probyn (1996) and geographer Antonisch (2010) claimed that belonging is not just about self-expression, but encompasses a desire for more than what is; a desire to be part of something greater than oneself. 


\section{Befriending and belonging}

Because of the focus on difference, scripted cross-cultural encounters are limited when it comes to bonding over similarities and forming intimate friendships. These cultural festivities also take place annually in Nelson, and are not designed to build the ongoing relationships that shape the rhythms and routines of everyday life or foster feelings-incommon. A practitioner from Victory Community Centre said,

I think that you can have all the formal structures that you want to help people to feel welcome and a part of a place, but it also needs to happen in that informal way. And it's easy for us to encourage people to, in this case Burmese, to participate in special events, either sharing their food or their dancing etc., but that's the sort of outward cultural expression and it doesn't necessarily bring people into a comfortable place with each other.

In aiming to bring people into a comfortable place with each other, practitioners were also facilitating inter-personal, informal everyday encounters. One Red Cross practitioner said,

We hold a few events per year, that's to bring our [local] volunteers and [former refugee] families together at somewhere to do something. The last one was down at the little train down at Tahunanui, and the next one is going to be down at Nature Land [the local zoo].

One of the practitioners also said, "We've got a programme that was starting, that hasn't started yet, and that is with Volunteer Nelson who are having a one-on-one sort of volunteering role. So, you know, it might be tree planting". As mentioned in previous chapters, planting or gardening is often used as a sociable befriending type of activity particularly in the UK where community allotments are common (Barnes, 2018; Biglin, 2020; Rishbeth et al., 2019; Room to Heal, 2018). In Barnes' (2018), Companion Planting Allotment Project in Bristol, children and adults from diverse backgrounds grow plants together as a way for parents to actively engage in their children's learning. On her website, Barnes said,

I called the project Companion Planting, which is a way of gardening where different plants are grown together that are of mutual benefit, because we feel that this idea echoes our ethos of bringing different people together to share skills and celebrate diversity in order to build stronger and healthier communities. 
Another practitioner added that, "The youth will go down and play volleyball. There is a group now getting together playing soccer, at the Girls College". Sport has drawn attention in refugee resettlement, as a way of bringing together people from diverse backgrounds who have a common interest, and who could participate in a shared activity together and foster a sense of collective identity (Mathisen \& Cele, 2020; McDonald et al., 2019; Spaaij et al., 2019). In health and sport researchers McDonald, Spaaij, and Dukic's (2019, p. 942) study with asylum seekers playing football in Australia, one participant claimed that,

When I came to this country, I just came by myself. I didn't know anyone here, so I think being, being a part of a soccer team and ASRC made me make friends and beside that even when we play a game, you still have to meet other people and have a conversation with another people. Another thing is a good opportunity to get to know a different area.

Whilst these activities enable individuals to become familiar with one another on a personal level, sociologists Spaaij et al., (2019) have cautioned that sport may not always be conducive to reciprocal exchange, and at times may serve to further assimilate former refugees into dominant cultural norms. Thus, there is a need to remain alert to the power imbalances that shape even the most convivial and friendly spaces.

Drawing on concepts of conviviality (Gilroy, 2006), and recognition-with-others (Askins, 2016; Noble, 2009; Wright, 2015), geographers Rishbeth, Blachnicka-Ciacek, and Darling (2019, p. 127) discuss these types of bonding or befriending schemes as a form of 'curated sociability', designed to boost confidence in marginalised or excluded individuals and enhance access to public spaces. They claim that, "The etymology of 'curation' is relevant here, deriving from the Latin curare "to take care". They add that, "Rather than simply providing organisational frameworks, the approaches we discuss are relational, considered practices of paying attention to the individual, which can make experiences of unfamiliarity less fearful". Thus, curation paves the way for more inclusive and hopeful ways of 'living together with difference' (Wise, 2005; Wright, 2015).

One Nelson programme that aimed to shrug off the lingering traces of formality that come with the act of curating, is a summer activity called 'Lark in the Park'. A practitioner from 
the Victory Community Centre said, "That is down at Victory Park. It relies on lots of volunteers to take turns [at providing adult supervision] and it's on Friday nights from five o'clock to seven thirty ... So that's another way those kids are mixing with other kids". Lark in the Park differs from other resettlement initiatives as it does not attempt to foster relationships through organised activities, but through offering a time and space for allinclusive, unregulated exploration, interaction, and play. Thus, it offers a more organic sense of place-making through happenstance or chance encounters. As mentioned earlier, these chance encounters may not always be positive, and may come with an element of risk. However, their free-flowing nature also creates space for an element of 'rupture' and 'surprise', and could pave the way for greater curiosity and enchantment (Geoghegan \& Woodyer, 2014; Wilson, 2017) and the formation of unlikely friendships (Askins, 2016). Such bonding initiatives are particularly valuable, as psychologist Lewicka (2011, p. 217) claimed that, "Strength of community ties has been consistently found to positively predict place attachment".

Becoming familiar and bonding with new places and people can help former refugees to feel more settled and may entice them to stay long-term. Sui Tin Men said, "In 2016 we visited Myanmar. I want to visit again, but not to live there. Living in Nelson is better". Ngun also said, "I'm happy here [in Nelson]", and Sui Sung said, "The future is Nelson". These comments reflected findings in labour and immigration researchers Searle, Prouse, L'Ami, Gray and Grunner's (2012, p. 108) resettlement study. The authors claimed that 97 per cent of former refugees had applied for or been granted citizenship in New Zealand after 10 years of entering the country as permanent residents. When asked why they chose to stay, "Most replied that they felt New Zealand was their home, they wanted to feel part of New Zealand society or because they had lived in New Zealand for a long time". Yet, simply being in a place or participating in existing activities does not necessarily lead to feelings of place-attachment or belonging. As sociologist Lefebvre (1974) and geographer Harvey (2003) claimed, equal access to urban life also involves increasing agency through enhancing one's ability to change the city and society; to be part of the making and (re)making of place. 


\section{7:3 Changing the city and society}

\section{Investment, nurture, and a feeling of pride}

On a local scale, the girls and women were changing the sensory landscape of their homes through playing traditional music, speaking Indigenous languages, displaying cultural objects, and cooking with familiar and comforting smelling spices. This was an important first step in place-making; however, there were significant barriers as to what could and could not be changed within the home, particularly for families who were renting from the government or private landlords (which included roughly half of the research participants). Due to restrictions that prevented renters from being able to remodel houses (or sometimes even hang pictures on walls) home ownership and the freedom (and prestige) that came with it had become an important goal for participants. Zai Zai claimed that, in the future, "I will work, saving money, buy a house ... When I'm older I need to have my own house. My own house”.

Unfortunately, purchasing property may not be financially viable for some time after former refugees arrive in New Zealand. Home ownership has also become an elusive dream for other working and middle-class New Zealanders in light of rising living costs and the current national housing crisis, which is significantly impacting the Nelson Tasman region (Community Action Nelson, 2019). This is problematic as home ownership has been found to be a consistent positive indicator of place-attachment, due to the stability, security, and freedom it provides (Brown et al., 2003; Lewicka, 2011).

Former refugee communities also faced the challenge of having to rent other important places, such as churches. The Chin resettlement practitioner whom I interviewed said, "It's the Baptist church, near the Court. We use that. Baptist church for Kiwi people in the morning, then we use in the afternoon. We share. We don't have our own church, so we just borrow. Hire". The girls and women were grateful for the provision of this building, yet their lack of ownership hindered the opportunity for any long-term investments and prevented them from making any major decisions about how rented places might be maintained and protected for future use. Such a lack of agency could diminish a feeling of stability (knowing that a landlord could force them out or sell the property at any time) and 
restrict them from freely expressing themselves and harnessing their creative potential to transform and alter their surroundings (Lewicka, 2011).

Yet, even without financial investment or ownership, there remains potential to alter place in meaningful ways. As mentioned in the previous chapter, all of the women (homeowners or not) had planted vegetable gardens in their backyards. Jenavi had also potted a row of flowers leading up the path to her front door, and Sui Sung had pointed out a vibrant cluster of flowers in her garden and described how she would like to plant similar bulbs in empty baskets that were hanging on the fence, to make the area feel brighter and more homely. The practice of gardening was beneficial as participants could create meaningful relationships with place through investing time and energy into cultivating the land. As mentioned in section 2:3, such investments can lead to a sense of ownership and belonging through the assumption that people have a right to enjoy the product of their labour (Hay, 1998; Porteous, 1976; Relph, 1976; Steadman, 2006).

Investing time and energy in gardening also contributed to a feeling of pride. In one of my research reflections I wrote:

Our interviews tentatively began with participants averting their gaze, moving slowly, and speaking quietly; unsure of how to act or what to say. It was clear that they were feeling shy about the interview process; however, when the conversation turned to gardening, these fragile encounters instantly adopted a vibrant new dynamic. The women were jumping up out of their seats and leading the way outside. They were commanding my attention, smiling, laughing, joking, and ready to teach me anything that I might possibly desire to learn about this place which they had planted, nurtured, and transformed. Their pride was written on their faces, and I felt it radiating in the spaces between us, tenderly interwoven with the magnificent canopies of green climbing over fences and spiralling down backyards (Field notes, 12/02/2019).

Geographer Morrison (2016, p. 104) claimed that, "Pride is unique among the emotions in the way it is tied to stake holding for one only feels pride (or shame) in people, events, or places in which one has a stake, through investment, ownership, or membership".

However, perhaps more salient than the time spent investing in these gardens (which alludes to a capitalist process of maximising one's returns and profiting from one's 
actions), was the time spent 'nurturing' and 'caring for' the land. This nurturing approach signifies a co-dependent, mutually beneficial relationship between people and place and centralises a process of connection (Bawaka Country et al., 2016; Buergelt et al., 2017). When speaking about a gardening project for former refugees in the UK, one practitioner said,

[Gardening] enabled them [former refugees] to grow their own food and so it meant they could reduce their costs, but it also meant that they got their hands in the dirt and they sensed that they'd arrived. A number of them described, actually I remember that sensory thing of connecting with the earth and growing crops and looking after them and everything, and, it really made them feel that they had arrived and they were connected now to the land. And that connection to the land for a lot of people, particularly from the rural areas, was really helpful.

Having the opportunity to plant trees and gardens thus encouraged former refugees to literally and symbolically put down roots in Nelson so that they could continue to grow, flourish, and feel as though they have a place to belong (Biglin, 2020; Coughlan \& Hermes, 2016).

\section{Becoming part of the fabric of place}

Equally important was the opportunity to invest in, nurture, and feel proud of one's neighbourhood and city. One way in which former refugees (and other residents) often achieve this is through employment or volunteering (unpaid community work). One of the resettlement practitioners claimed that, "All the refugees that I've ever come across always want to work and get employment". Jenavi said,

The first thing is I really want to [learn] good English ... When I think I have very good English I want to do job. And also, I want to do volunteer to do a lot of things ... I like volunteering just once a week or like that. Look [after] old people, and also, um, second-hand shop to do ironing, a lot of things ... I think New Zealand people is very nice and also, they do, a lot of people do volunteer. How good volunteer is!

Employment and volunteering enables individuals to actively contribute to and change the city and society in various ways, the first being to raise the profile of diverse ethnic groups and make multiculturalism more visible. Askins (2016) claimed that this visibility can 
support the emotional need for recognition, identifying former refugees as hardworking contributing members of society. It can also normalise the sight of different coloured bodies in public spaces and enhance the type of convivial culture that Gilroy (2006) spoke of, whereby superficial racial difference becomes banal to the point that it no longer has the power to discriminate.

The second way that employment enables change is through practice, whereby individuals can share their knowledge and skills, establish new relationships and networks, and enhance social capital (Lewicka, 2011). Then there is the product of one's labour, where the construction of a new building redefines the contour of the skyline, or the cooking of traditional cuisine in a new restaurant alters the smellscape of the streets. These subtle changes weave newcomers into the fabric of a city and enable them to see themselves 'mirrored' in their environment (Mathisen \& Cele, 2020). In this manner, they are able to leave a mark and become part of the material and historical narrative of place.

As geographer Antonsich (2010, p. 648) claimed, "This sort of economic embeddedness matters not only from a material perspective, but also in relation to make a person feel that $\mathrm{s} /$ he has a stake in the future of the place where s/he lives". For former refugees who have been torn from the fabric of their homeland, this weaving or embedding of the self into a new social, economic, and physical landscape may be pivotal in re-orientating themselves in time and space and reaffirming that they have some purpose or meaning in the world. According to feminist hooks (2009), such meaning is precisely why individuals strive to find a place to belong; to create "a life worth living" (Antonsich, 2010, p. 649).

Employment or voluntary work is not the only way to change a city or society, and creative art projects, music installations, and public activism also play important roles in enacting sociospatial (and political) change. Unfortunately, participants were unable to pursue many of these activities due to their busy domestic lives, and for the most part made do with altering their homes and gardens (although they all hoped to find work once their children were older or their English had improved). One obvious exception was the public exhibition of the girls and women's artwork from this research. Whilst their paintings were only on display short-term, for a while they had a presence in the city that wove their 
personal narratives into the wider fabric of Nelson, and enabled them to feel a sense of pride and self-worth (see section 4:2).

\section{Negotiating tensions of belonging}

Unfortunately, changing the city and society can create tensions with long-term residents, who may fear that the places to which they feel attached are becoming unrecognisable (Law, 2001; Kale et al., 2019; Waite et al., 2014). The perception of newcomers as a threat to a place or local way of life can lead to long-term residents becoming fiercely protective of 'their' neighbourhood, city, or country and hostile to development. Sociologist YuvalDavis (2011, p. 5) said, "In most extreme cases people would be willing to sacrifice their lives - and the lives of others - in order for the narrative of their identities and the objects of their identifications and attachments to continue to exist."

If long-term residents are not also supported to adjust to the sociospatial changes that arise with immigration and resettlement, then it is easy for resentment between different groups to grow. If left unchecked, such resentment can lead to further discrimination and exclusion toward newcomers and compound ongoing resettlement challenges. Geographers Mathisen and Cele (2020, p. 1) therefore claimed that, "Former refugee's belonging needs to be understood as relating to other people's understandings of their right to belong".

Yet, belonging is not only contested between long-term residents and new arrivals or 'insiders' and 'outsiders'; it is in a constant state of flux (Massey, 2005; Wright, 2015). Geographers Rishbeth et al., (2019, p. 132) claimed that,

Recognising the footprints that are made by various user groups, from asylum seekers and refugees to more established residents, involves recognising that public spaces are constantly changing. This responsiveness is integral to resisting a normative pressure for the strange to be assimilated into the known.

Other researchers who are resisting the pressure to assimilate the strange into the known are conceptualising belonging as an emergent process of co-constituting and co-becoming (Bawaka Country et al., 2016; Wright, 2015). In these ways, each object or entity (human and non-human) plays their part in making and remaking place, together. Geographer Wright (2015, p. 402) claimed that, 
There is no pre-existing world to be reflected, to be belonged to. Rather, the performances, practices and affects of belonging (re)make that world together, they bring it and themselves into being in different ways. This could be conceptualized as belonging as emergent becoming, a belonging based on relational ontologies.

Continuing with this vein of thought, belonging could also be conceptualised as an act of harmonising or attempting to harmonise with one's surroundings. In harmony (it may help to think through the metaphor of music), diverse elements are drawn together into a dynamic relational process whereby each retains its own value yet compliments the other and becomes something more in a lively and nuanced texturing of time and space. Psychologists Di Fabio and Tsuda (2018, p. 3) claimed that, "Harmony is an emergent order; it is not uniformity; it is a holistic perception; it is a dynamic equilibrium ... Harmony exists in a reality that is to be shaped every time". Interdisciplinary researchers Buergelt et al., (2017, p. 290) also argued that, "Harmony creates balance, union, synthesis, calm, tranquility and peacefulness ... accordingly, living in harmony is synonymous with health and well-being".

Thinking of physical entities harmonising in the symbiotic way that musical notes can do, highlights the curation, creativity, and work involved in an emergent process of belonging. In the often-quoted words of philosopher Plutarch, "Music to create harmony must investigate discord". Belonging is also premised on a process of investigation, of "trialand-error' (Fullilove, 1997). In this manner, there is an inherent messiness and frustration to it, and when things aren't working out well, or are taking longer than one expected, individuals may feel like they don't belong. One practitioner said, "They [former refugees] are learning the language and, particularly for older people, the longer it takes the more frustrating it is. Over time, unless things click into place it becomes very difficult”.

Thus, in addition to time, active engagement, and negotiation with others, feelings of belonging and place-attachment require a great deal of patience, which anthropologist Procupez (2015, s63) defined as, "Waiting while working to make something happen". Whilst the act of being patient or waiting may seem passive, geographer Kindon and I have previously drawn upon Procupez's (2015) work to argue that, "Passively and futilely waiting with no end in sight is very different to actively waiting and working to bring 
about change or accomplish a goal ... Such objectives are often only obtainable with a certain level of hope and patience" (Kale \& Kindon, 2020, p. 13; see also Jeffrey, 2010 and Pottinger, 2017).

Changing the city and society is a complex process, and newcomers leaving their mark will remain contested in many ways as individuals try to figure out how best to live together with their differences; how to harmonise. Perhaps the most optimistic way that change can be framed in this context is as an opportunity. One of the resettlement practitioners claimed that change is not only inevitable, but necessary if places are to accrue the specific qualities that an individual comes to holds dear. He claimed that the places we become attached to could "be anything":

It can be a street, you know, you walk up that street every day ... It can be the tree. Those are the real treasures I think. Then they go and knock it down, or cut it down! But I always say in that situation: It had to have changed from something to become that place that you remember it as. So, those people who took fancy in it then would have though the change is not right. But for you, when you experienced it, it was like well this is fantastic!

This point is particularly salient as it recasts change in a hopeful light, replacing fear of the unknown with the possibility for new opportunities. As mentioned in Chapter Five, possibility and opportunity are pivotal to place-attachment and wellbeing, as they provide what $\mathrm{Mu}$ Pli described as a way of 'getting to the future'. This focus on hope is not to say that feelings of loss are unimportant, but rather to suggest that the pain that accompanies loss is not necessarily all-encompassing. Even in the most upsetting of circumstances there may be space for more positive encounters and experiences to emerge.

\section{7:4 Key learnings}

Returning to the research sub-questions; repetitive encounters with multisensory stimuli influence individual feelings of place-attachment and wellbeing over time through fostering a sense of familiarity. As individuals regularly engage with their physical surroundings, strange-looking foreign-textured environments become recognisable, predictable, and easier to navigate (Fullilove, 1997; Seamon, 1980). This enables 
individuals to feel a greater sense of control over their movements and actions and a greater sense of safety and stability in place (Hage, 1997). Yet, places are also constantly changing and in addition to becoming spatially orientated, it is important for newcomers to familiarise themselves with a place's daily social routines and seasonal cycles if they are to maintain feelings of security as the warmth of spring bleeds into the blistering stickiness of summer and the shivering chill of winter.

Regular encounters with sensory others also helped to strengthen feelings of placeattachment and belonging through fostering familiarity and enabling individuals to bond and form new friendships. However, familiarity is only one step in the process of placeattachment and belonging. Former refugees (and other marginalised individuals) also need the opportunity to actively invest in and nurture their environment; to change the city and society (Harvey, 2003; Lefebvre, 1974), and become woven into the fabric of place. It is through these active practices of 'doing belonging' (Mathisen \& Cele, 2020) that placeattachment moves beyond an instant feeling of attraction or a positive emotional response to one's environment; and entails the formation of a meaningful committed relationship over time (Hay, 1998; Steadman, 2006).

Distinguishing between a fleeting feeling of place attraction and more sustainable feelings of place attachment is important, as these experiences meet different needs and differently impact wellbeing. Whilst attractive places (i.e. places that instantly feel homely, safe, beautiful or enchanting) can provide space for rest, reflection, and emotional renewal, the places to which one feels attached have the added benefit of fostering a sense of stability, identity, and belonging. Attractive places may lead to feelings of attachment, as they are likely to draw people back and enable familiarity and investment over time, though not every attractive place will become meaningful. An exception to this process may exist when a place holds symbolic meaning for an individual which links them to previous places and elicits a feeling of nostalgia (Akhtar, 1999; Volkan, 1999). In this case, an individual may feel as though they have already invested significant time and energy into a place through their memories of past attachments, as was evident in Chapter Five with Zai Zai's immediate connection to the acorn tree that represented her childhood. 
The final point which I want to stress from this chapter is the difficult (yet rewarding) work involved in belonging somewhere and becoming attached to place. The endless hours that the girls and women devoted to familiarising themselves with Nelson, learning English, trying new food, meeting new people, and tending to their gardens was crucial in fostering strong durable relationships with place. Likewise, the "hundred-and-one things" that practitioners and volunteers do to help former refugees to resettle in New Zealand cannot be undervalued. These exciting, frustrating, difficult affective processes, and the joy, sweat, and tears that are shared, are ultimately what bind individual entities and imbue place and life with feeling and meaning. 


\section{Chapter 8: Feeling Well in Place}

Drawing upon the key learnings from the previous chapters, this chapter argues for the theoretical and practical emplacement of refugee wellbeing. Specifically, I suggest that

refugee mental health needs to be recontextualised within the sensing feeling human body and the wider sociospatial, temporal, and more-than-human relationships, networks, and flows which shape the subjective emotional experience of resettlement. In what follows, I discuss participants' assessments of current mental health care in Nelson. I then build upon their earlier comments and artwork depicting the places and activities which make them feel (un)well, and explore the where, when, and how of refugee wellbeing, before questioning who is responsible for implementing and maintaining a decentralised relational wellbeing approach. In this manner, I tentatively begin to bridge the gap between resettlement theory, policy, and praxis, beginning with an ethics of responsibility and care.

\section{8:1 Emplacing refugee mental health}

\section{'The dark underworld'}

As mentioned in Chapter One, refugee mental health care in New Zealand primarily focuses on trauma recovery (New Zealand Red Cross, 2018; RASNZ, 2018); however, only a small number of individuals are diagnosed with a trauma-related illness such as PTSD (Javidi \& Yadollahie, 2012; McLeod \& Reeve, 2005). Many emotional and psychological health issues arise from post-settlement stressors such as being in an unfamiliar place, not knowing the language, facing unemployment and racism, and being separated from loved ones (Fullilove, 2004; Rosbrook \& Schweitzer, 2010). As highlighted in the previous chapters, additional challenges arise from uncomfortable foreign smells, sounds, and flavours, a new climate and terrain, and a new pace-of-life. These stressful everyday experiences can make former refugees feel out-of-place, disorientated, and homesick as they attempt to resettle. If left unchecked, they can also accumulate over time and lead to serious health issues such as depression. 
Despite participants in the Nelson Multicultural Council (2012) report and Ward et al.,'s (2018) study raising concerns over increasing mental health issues for former refugees in Nelson, little progress has been made to implement new solutions. At the time of this research, there were few mental health services available for residents to access in Nelson, and those that did exist were not being utilised by former refugees due to personal and cultural understandings and a lack of local knowledge around how to cope with diverse health-related issues. Language also posed a barrier, and one practitioner said, "I think it's been a real challenge around our primary health service, going to the GP, making appointments, just the bureaucratic system we've got and needing to organise an interpreter".

When I asked participants if they had people with whom they could speak if they felt upset, Mu Pli said,

At school there are like some website that the teacher told us we can go to, especially for like sexual abuse or if you are feeling depressed or need a counselor. They do tell us we can look up in internet and they do give us sources like paper source. I'm not sure where I keep them now, but I could just search up again.

This elusive website and some misplaced papers with the names of local counselling services was the only suggestion made as to where participants might access professional help, and Mu Pli claimed that she had not used these services and that Kayan youth and adults generally preferred to talk to other members of the community rather than strangers. Little Ruth said that the Chin community also, "Just talk to each other, help each other". Zai Zai agreed, saying that, "We help each other, go to their house and pray. And the community give them ... If your family in Myanmar one of them pass away and you feel really sad, then we go to our community people, we go and pray. And some, not a lot, we bring some money”. Supporting one another through prayers and financial aid was beneficial insofar as it enabled individuals and families to cope with unexpected or overwhelming events. It also helped them to feel a sense of the cathartic social and spiritual healing that was discussed in Chapter Six. However, upsetting personal experiences and memories were not necessarily being shared in these contexts, and when 
they were, there was evidence of stress being transferred to other community members. One resettlement practitioner (who was also a former refugee from Chin State), said,

They [other Chin community members] talk to me. I just listen, spend one or two hours, sometimes on the phone. Sometimes I visit them. They call and have a problem, 'Oh, come and visit', so I visit them. And I don't know who to talk [about] that to. That's why I need support too. Sometimes it's too much and sometimes I get stressed too. I just listen and whatever I can support I support them ... And sometimes I talk to their husbands too. Sometimes it is difficult for me to, sometimes my position is a little bit dangerous when I talk to their husbands. Sometimes they can get angry. But I say to them, 'I just want to see that your family are happy, that's the reason I come here, to see what I can do to help. To support your wife as well as you'. But when there is trouble and anger we say things that we can't control, and we can feel regret ... So, you know, I'm a social worker. But I'm not. You know what I mean? Like a social worker, but just a friend. I'm not social worker that can deal with the problem and everything, but my position is quite hard.

The stress of filling the role of a social worker without the necessary qualifications or supervisory support system, and whilst trying to maintain the role of a friend and confidante, is troubling. Individuals in such a position may find their own emotional and mental health to be at risk. Intervening in incidents of family aggression and violence also places these community members in situations where they themselves could become victims of abuse, and where abuse continues to thrive as no professional intervention or treatment is sought.

Sui Sung claimed that community support was also problematic as there was a lack of protocol around ethics and confidentiality which inevitably led to community gossip, judgement, and stigma. She said, "Sometimes, yeah [talking to friends] is good, but sometimes it's ... oh. Sometimes I'm talking to you, same this person Chin ... she is talking ... oh a secret this one! Tell everybody”. For anonymity, several of the women mentioned that church pastors were trustworthy people to speak to; however, the Chin resettlement practitioner claimed that,

In Nelson we don't have a pastor so that is why it is difficult. The Chin Glory Evangelical Church, now we are trying to get a visa for the pastor from Burma. And 
it's taken quite long now. I don't know when he is coming. We still waiting for Immigration to say yes. We really need that support. The New Zealand Government also needs to understand that we don't have a pastor here, no way to get him here, we need support. Especially from our own church. We need a pastor ... So, we can talk, who can support us when we are upset or things. Otherwise here we just support each other.

The lack of refugee mental health providers, internal community tensions, and a breakdown of traditional cultural and religious support structures was problematic. Several of the participants discussed times when they had felt sad, worried, and stressed in Nelson, and one individual claimed that a neighbour from the Chin community had committed suicide.

Local resettlement practitioners also mentioned that they had encountered or heard about former refugees suffering from varying levels of stress, anxiety, or trauma. The practitioners claimed that many former refugees were only accessing professional care when it came to 'breaking point' and were ending up in the local hospital which was unequipped to deal with such cases. For example, one practitioner said,

Mental health is an issue that is coming up a lot these days. And um, through the

District Health Board, well it's not really their puppy either so ... it's sort of a, well there is a real gap out there ... With the Burmese community, they sort of keep [their personal stress and trauma] quiet. For the males it manifests in depression, alcoholism and abuse, which all happens at home, unseen. Whereas with other groups it seems to spill out all over the place. But with the Burmese it's sort of like the dark underworld, you don't really know what is going on.

Other practitioners claimed that the wives and children of traumatised men often bore the brunt of this abuse, and that the suffering of female former refugees tended to be exacerbated by their heavy domestic responsibilities which often kept them isolated in their homes and distanced from potential support groups. Another practitioner said,

I do know that alcohol can be an issue, particularly for the men. Also, domestic violence is ... I have been involved with a couple of families where that has been an issue. And I think the underlying thing is that stress and loss of identity and fear; 'how are we going to survive'? 
These gendered dimensions are not unique to former refugee families, but, as previously mentioned, reflect nationwide issues of mental health, substance abuse, and domestic violence (James, 2010).

My findings suggest that Nelson's current mental health care system is inadequate in supporting former refugees. I therefore reaffirm the call made by participants in Ward et al.'s (2018) study for 'alternative therapies' in the health system. To alleviate some of the pressure upon individuals, communities, and the Nelson hospital, I argue that more preventative healthcare approaches are needed that aim to minimise everyday feelings of stress and anxiety and maximise the experiences that enable individuals to feel well and happy. This focus on prevention has also recently been identified as a necessary approach to broader health and wellbeing in New Zealand (New Zealand Government, 2018a), and this research contributes to this important nationwide discussion.

\section{Feeling well through bodies and places}

To prevent or minimise individual feelings of stress and anxiety, an ontological shift is needed. This shift needs to move from positioning refugee mental health as a psychological problem to be fixed through counselling or medication, to a questioning of what it is that is causing or exacerbating feelings of stress and anxiety in everyday life and actively addressing these causal relationships. As mentioned in Chapters Two and Three, the mind and body are not separate entities, and individual memories and thoughts emerge through one's embodied multisensory interactions with one's surroundings (Andrews et al., 2014; Butler, 1988; Damasio, 2000; Merleau-Ponty, 1962; Relph, 1976; Thrift, 2008). There is therefore a need to contextualise mental health within the sensing feeling human body, as well as within the physical, social, virtual, and spiritual places with which individuals engage on a daily basis (Atkinson et al., 2012; Conradson, 2005; Gesler, 1992). In other words, mental health researchers and practitioners need to recognise that pathological thoughts and memories are viscerally and proprioceptively felt and that, "Place matters' to health and health care" (Andrews et al., 2014, p. 211).

To corporeally and geographically spatialise mental health, I have drawn upon holistic concepts of wellbeing as theorised by Indigenous communities and researchers (Bawaka Country et al., 2016; Durie, 1985; Pere, 1997; Wilson, 2003), and more recently by 
geographers (Atkinson et al., 2012; Kearnes \& Andrews, 2010; Pain \& Smith, 2010). The potential of holistic wellbeing is still being realised in refugee resettlement literature (Biglin, 2020; Coughlan \& Hermes, 2016; Dyck \& Dossa, 2006; Rishbeth et al., 2019; Sampson \& Gifford, 2010), and arguably has yet to have a substantial influence on mental health care practice for former refugees in Nelson. However, this is not to say that the wellbeing turn has gone unnoticed by local practitioners, and one practitioner whom I interviewed said, "I think the Māori model, for me anyway, the Māori model of health is so much more relevant. I'm not a great fan of the biomedical model. And so, I can see a lot of synergy here [in refugee wellbeing] with Te Whare Tapa Whä". Rather, the question remains as to how to practically apply such models within the resettlement context.

Acknowledging that the wellbeing of former refugees (and other people) is emplaced in everyday multisensory experiences, events, and interactions is significant as the girls and women I worked with commonly related their personal feelings of stress, anxiety, and worry to their sociospatial encounters. As evident in Chapters Five, Six, and Seven, they felt uncomfortable with a different-sounding language, confused by unfamiliar streets, nauseous from strange-tasting foods and pesticides, overwhelmed by fast-moving traffic and the pace of urban life, cold due to a new climate, and upset by loud noises which triggered memories of violence and warfare. Individually, these unfamiliar, uncomfortable, and painful feelings could alter one's affective emotional state (Damasio, 2000; Hinton et al., 2004; Nguyen et al., 2018; Thrift, 2008). Together, as they accumulated over time, they significantly impacted how individuals related to and identified with place, leading to feelings of not-belonging and missing home.

To neglect these multisensory interactions is to obscure the affective relational origins of mental health (Andrews et al., 2014; Atkinson et al., 2012; Hinton et al., 2004), and to overlook ways in which stress could be minimised on a day-to-day basis. It is also to hide the positive place-based experiences that might serve to enhance wellbeing (Kearns \& Andrews, 2010; Pain \& Smith, 2010). For instance, the girls and women spoke of feeling happy when they viewed stunning landscapes and submerged their hands into the dirt, nurturing their gardens. Their curiosity was aroused by beautiful flowers, differentsounding birdsongs, and leaves that stayed green all-year-round. They felt free while praying, singing, and dancing, and appreciated the warm nostalgic feelings evoked through 
cooking their favourite dishes from home. These everyday multisensory experiences were therapeutic, healing, rejuvenating, empowering, and central to the construction of identity, belonging, and hope. Whilst such feel-good experiences are not a quick-fix for depression or trauma-related mental health issues, they are significant in regulating one's emotional and mental state and re-thinking what it might mean to live a happy and healthy life. Exploring a state of 'contentment' or 'wellness' (Pain \& Smith, 2010) is thus equally as important as understanding what makes an individual feel ill. Importantly it could provide a much clearer idea of how wellbeing might be realised in practice.

\section{The influence of distant others}

In addition to contextualising refugee mental health in the sensing feeling body and local environment (in this case Nelson City), it is also necessary to consider how individual wellbeing might be influenced by distant people, places, and events (Andrews et al., 2014). As discussed in Chapter Five, nostalgic and fearful sensory memories can connect individuals to their homelands and other important places and prompt them to enact the past in the present (Blunt, 2003). Through simultaneously inhabiting multiple times and places, the distant 'then and there' becomes mapped onto the proximate 'here' and 'now' and viscerally and affectively felt through loud noises, strong wind, rumbling stomachs, and wet salty tears; or through the taste of familiar spices, the warmth of the sunshine, or the memorable texture of an acorn.

Many of the homely practices that participants remembered and re-enacted in Nelson also relied on the physical import of international commodities. The women shopped at local Asian food stores for ingredients to cook traditional dishes, located imported seeds to grow familiar vegetables, and brought with them various material objects and clothing that were entwined with the identity of being Chin or Kayan. Whilst these objects were physically used and experienced in Nelson, they were produced elsewhere and imbued with distant labour economies, trade regulations and a unique ethnogeographic heritage, indirectly linking daily practices of wellbeing to wider spatial migrations, networks, and flows. This transactional web has been described by geographers Amin (2002) and Massey (2004) as a politics of connectivity. 
Increasingly, this politics of connectivity is also being played out online, through social media and other information sharing news sites and platforms like YouTube (which Mu Pli mentioned utilising during her moments of solitude). Resettlement researcher Marlowe (2019, p. 179) claimed that, "Social media effectively collapses physical geographies of distance through incorporating increasingly intimate transnational interactions and political commitments into everyday lives". As mentioned in section 5:3, the girls and womens' frequent videocalls abroad helped them to maintain important relationships, but also evoked fear and worry for the safety of loved ones who remained trapped in conflict and poverty. Their wellbeing was thus intimately intertwined with the wellbeing of others and the volatile politics of Myanmar's civil war, as well as being linked to the international resettlement system which they viewed as a pathway to family reunification. Thus, as Marlowe (2019, p. 180) claimed, "Refugee settlement is, for many people, an ongoing transnational experience". He added that, "For many refugees, regular and reliable access to transnational networks is a growing and an integral part of settlement that sustains and supports people's overall well-being".

Online communication has also indirectly influenced refugee wellbeing through the proliferation of (mis)information regarding the 'refugee crisis'. Whilst individual Nelson residents were responsible for the racist, exclusionary behaviour that they directed toward former refugees, and whilst their threats and actions were viscerally felt by the girls and women locally, such anti-refugee sentiment has emerged in New Zealand through the reverberations of politics abroad. Accessible international news and the far-reaches of social media into cities and homes have played a role in rising nationalism and protectionism around the world, manifesting in local contexts as 'bodily hurt' (Waite et al., 2014), and fractured sociopolitical relations. Thus, whilst the symptoms of stress, trauma and suffering are visible through individual emotions, thoughts, and actions, the roots run much deeper and are inextricably entangled in multiple places at multiple scales. 


\section{8:2 Harmonising with one's surroundings}

\section{Finding a comfortable rhythm}

For participants, feelings of stress and anxiety were not only spatial but temporal, evoked through displacement from familiar habits, rhythms, and routines, and emplacement in unfamiliar time-structures and place cycles (Edensor, 2010; Harvey, 1994; Lewicka, 2011; Parr, 2010; Seamon, 1980). This temporal dimension was evident in their discussions about feeling out-of-sync with the different seasons in Nelson, the cicadas singing at a different time of day, and the fast pace-of-life in the city. Whilst some of these experiences evoked a mild sense of disorientation and nostalgic memories of home, others, in particular the paceof-life, had a profound destabilising effect and continued to influence how participants engaged with different places decades after their initial resettlement. Thus, it is not only the rhythms of time, but the duration of different rhythms and duration of emotional responses to these rhythms that require attention (Adam, 1998).

As geographer Seamon (1980) and historian Parr (2010) suggested, becoming used to new seasonal cycles and social routines is difficult due to these patterns being ingrained in individual memories and bodily movements and performed almost subconsciously. Yet, to do and feel well in a new sociospatial environment, there is a need to adapt to the local time-structures and seasons and to replace old habits and routines with new ones. Parents have to take their children to school at a specific time, employees must arrive at and leave work at a certain hour, and many New Zealanders will need to alter the way that they care for their homes in the winter to prevent mould and dampness. Without making these changes, individuals will likely find themselves under-educated, unemployed, and possibly falling ill as the temperature drops.

Whilst such adaptations are difficult and not necessarily desirable, not all habits and routines must be changed, nor will every resident need to adopt the same uniform rhythm as their neighbours. Just as individuals negotiate the use of space with others and use space in nuanced ways, they also negotiate the use of time. As geographer Edensor (2010, p. 5) said, "The body also produces place as well as fitting in with it, and it may not keep in step or synchronise with regular beats". These temporal negotiations bring diversity and multiplicity to a city, meaning that residents do not simultaneously flow together in a 
singular coordinated rhythm (in the way that schooling fish do), but rather create overlapping beats and pulses that lead to everyday life being 'polyrhythmic' (Edensor, 2010; Lefebvre, 2004; Massey, 2005; Potts, 2015). This multiplicity can be harmonious, with the rhythm of the waves complimenting the birdsongs and the laughter of children to create a peaceful ensemble; and at other times discordant, with partygoers playing music into the early hours of the morning and disrupting the silence that other residents and sleeping wildlife might crave.

One of the ways in which the girls and women achieved some control over time (and space) was through their moments of solitude. Being alone (or at least distanced from society) meant that individuals no longer had to fit into the usual temporal structures and timetables, and could take 'time-out' to pause, reflect, rest, and relax. This retreat effectively enabled them to slow down for a while, to escape the fast pace of the city and the demands of community, family, social and domestic life. At other times it may have enabled them to speed up moments of dullness and monotony - to become more focused, creative and productive (Long \& Averill, 2003).

Such temporal control was important for those women caring for young children, juggling domestic life and other social commitments, and hoping to find employment. In their article 'Shadow Times: The Temporal and Spatial Frameworks and Experiences of Caring and Working', sociologist McKie, health researcher Gregory, and geographer Bowlby (2002, p. 897) argued that, "An enhanced recognition of the fluidity and praxis of caring and gender is necessary to support the evolving roles of parents, especially mothers who combine caring and working”. In addition to gender, unique cultural, and rural / urban, perceptions of time were significant, and these intersecting positionalities require greater attention as to how time might be structured differently and negotiated between unique individuals.

The subjective un-structuring and re-structuring of time reiterates calls by geographers Marston et al., (2005), Thrift (2008), and Jones (2009) to acknowledge that, whilst time always objectively flows forward (Amin, 2004; Hawking, 2009; Massey, 2005), life is not subjectively experienced by individuals as one fluid sychronised motion. Part of feeling well in place is therefore about finding a comfortable tempo, pace, or rhythm through 
which one can move, whilst also learning how to harmonise with the rhythms and routines of other individuals, wider social time-structures, wildlife, and cycles of daylight or the changing seasons (Buergelt et al., 2017; Edensor, 2010). As mentioned in Chapter Seven, harmony is not about uniformity, it is a 'dynamic equilibrium' (DiFabio \& Tsuda, 2018), an emerging interactive process through which unique sociospatial and temporal entities can find ways of being together, becoming together (Bawaka Country et al., 2016), and living together with difference (Wise, 2005; Wright, 2015).

On the other hand, as geographer Harvey (1994) mentioned, intentionally disrupting this harmony and adopting unconventional time-space practices may be an effective form of resistance and protest. Geographer Edensor (2010, p. 12) claimed that, "There is always the incipient tendency for entropy, subversion, breakdown and thus, arrhythmia", and also for what he called, 'rhythm resistance'. He continued to say that (p. 14), "In a polyrhythmic assemblage, rhythms influence each other, sometimes achieving eurythmia, where stability persists, and sometimes, arrhythmia, where they jar and clash". Returning to the idea that discord is a necessary part of creating harmony, arrhythmia may be a necessary part of achieving stability or a level of personal contentment and wellbeing, and is an important part of everyday placemaking and belonging.

\section{Sensory modulation}

Because bodies can become 'overwhelmed' (Thrift, 2008), people can become 'anchored' or stuck for a while (Jones, 2009), and there will be days when individuals feel or choose to be out-of-sync or arrhythmic with their surroundings, wellbeing needs to be considered as an ongoing emergent process and always in a state of flux (Atkinson et al., 2012). To help individuals to feel well as their surroundings change, several practitioners mentioned the value of 'sensory modulation' as an individual coping technique to manage stress and anxiety. One of the occupational therapists said, "In a very, very, simplified way, sensory modulation is that ability to use our senses to help modulate how we feel, and how we respond". She added that,

We want to give people that sense of control. It's about helping them develop that. I've identified what is helpful for me, so then I can do that myself. And not in any way to put down medication, because medication has an important role to play, but this is 
often the feedback we get, you know, this is something that I can do. It's my individual thing and I can share it with people in my life.

Most individuals modulate (or alter) their multisensory environment subconsciously through common everyday practices (Champagne, 2004). For instance, during our interview, Little Ruth made cold drinks to cope with the summer heatwave and claimed that, "I'm going a bit crazy, that's why we have a lot of ice and stuff [to cool down]". Meanwhile, Sui Tin Men said, "I like cleaning, wiping, doing the vacuuming. I feel more comfortable when my home is clean, then I can relax". One of the resettlement practitioners also mentioned that modulation practices can initiate or become part of one's daily habits and routines, subconsciously ingrained in the way that they interact with and create familiar places. She said, "I have a certain routine in the morning that helps me alert myself. It's different from the one I use at night. Now I don't think I'm doing sensory modulation, but I am, you know, I turn the lights down".

These mundane everyday actions entail a type of environmental therapy, whereby individuals are identifying uncomfortable or harmful aspects of their environments and altering these settings and their behaviours to treat associated physiological and neurological states and improve their lived spatiotemporal experiences (Randolph, 1987). However, individuals suffering from stress or trauma can be less attuned to their sensory needs and responses (Champagne, 2004). In these cases, there may be a need for some form of intervention to help former refugees to identify their sensory preferences and create prevention strategies that draw upon these preferences in times of anxiety, stress, or demotivation (Champagne, 2004; Te Pou o te Whakaaro Nui, 2018). District Health Boards in New Zealand (which are funded, monitored and managed by the government) offer sensory modulation as part of their mental health and substance abuse facilities ( $\mathrm{Te}$ Pou o te Whakaaro Nui, 2018); however, these services are not accessible to former refugees (or other New Zealanders) without a medical referral and could be (and arguably should be) more accessible within resettlement practice. 


\section{8:3 An ethics of responsibility and care}

\section{Placing wellbeing on the government agenda}

One outstanding question in regard to this nuanced everyday approach to wellbeing, is who is (or should be) responsible for supporting, funding, implementing, and maintaining such a decentralised relational approach? In the national He Ara Oranga wellbeing report, the New Zealand Government (2018a, p. 10) claimed that,

New Zealand's mental health and addiction problems cannot be fixed by government alone, nor solely by the health system. We cannot medicate or treat our way out of the epidemic of mental distress and addiction affecting all layers of our society. We need to ensure practical help and support in the community are available when people need it, and government has a key role to play here. But some solutions lie in our own hands. We can do more to help each other.

This statement raises three salient points, the first being that, "Government has a role to play here". Because society and sociospatial and temporal structures impact individual lived experience, then mental health and wellbeing should not be the sole responsibility of the individual. Governments rightly need to question the role that they play in contributing to residents' and citizens' feelings of stress and anxiety, and what they can do to enable diverse individuals to feel safe and well in place.

This responsibility has been conceptualised in feminist and geographical research as an 'ethics of care' (Askins, 2016; Fisher \& Tronto, 1990; Lawson, 2007; Popke, 2006). Feminist theorists Fisher and Tronto (1990, p. 40) claimed that caring is,

A species activity that includes everything that we do to maintain, continue, and repair our 'world' so that we can live in it as well as possible. That world includes our bodies, ourselves, and our environment, all of which we seek to interweave in a complex, life-sustaining web.

Geographer Popke (2006, p. 506) claimed that, "A care-centered theoretical perspective is thus premised on a relational conception of subjectivity, which stands opposed to the autonomous rational subject of individual rights and responsibilities". He added that (p. 507), "The key question is perhaps how an ethics of care can instil a sense of responsibility 
not only toward those with whom we have caring relationships, but also toward different and distant others".

Unfortunately, governments do not always accept responsibility for their role in civil wellbeing or provide the necessary resources to practically support the public health policies upon which they campaign. In Europe, geographers Sointu (2005) and Pain and Smith (2010, p. 89) have argued that wellbeing often becomes politicised through, "The neoliberal agendas of European governments who are keen to assign more responsibility for welfare to individuals". These concerns extend to New Zealand, where government funding and support for refugee resettlement and mental health services is limited and much of the care falls upon underpaid overworked practitioners and local volunteers. In speaking about the diverse Nelson clients with whom he worked in the mental health sector, one practitioner said,

It used to be that referrals were just from secondary mental health services... So we were only taking severe mental health, the top five per cent, and we've now been invited - I might add without any additional resources - to expand to moderate, which is the top twenty per cent. So, we are being asked to increase our services by a factor of, I don't know how many per cent, without any more resources. The usual story. Do more, on less.

However, governments can (and some do) care. Despite their many tendencies to disempower and harm individuals, attempts have been made by particular state leaders to welcome refugees. Over the past decade, the United States, Canada, Australia, New Zealand, and several countries in Europe have taken an active role in collaborating with local refugee resettlement and wellbeing initiatives, and in working together to promote the 'International Welcoming Network'. The aim of this network is to implement practical cross-cultural initiatives to strengthen community relations and to, "Create a world where everyone feels at home" (Welcoming International, 2020). Such international, multi-scalar approaches are vital to address the complex geopolitical events and networks of global, national, and local displacement and resettlement. 


\section{Strengthening social support networks}

The second salient point from the excerpt above is that, "We can do more to help each other" (New Zealand Government, 2018a, p. 10). This assertion may involve increasing recognition of, and with, diverse others (Noble, 2009; Wright, 2015), fostering convivial social spaces (Gilroy, 2006), enhancing practices of befriending (Askins, 2016), and increasing social capital (Kale et al., 2020; Strang \& Ager, 2010). In current New Zealand resettlement practice, social support networks generally operate through the Red Cross and its local volunteers, as discussed in Chapter Seven. The recently implemented CORS programme plays a similar role in orientating newcomers in place and connecting them to local services, as do local ethnic communities and religious groups.

Meanwhile, initiatives like Stress Busters in Nelson provide extra care and support for refugee wellbeing through stress reduction. One of the Stress Busters practitioners said,

There were quite a few [former refugees] being referred to the hospital and they felt like they couldn't cope with the numbers. And that's when the idea came up to have an intervention group where we would teach them skills and strategies to manage stress and anxiety. So, that's the focus of those groups.

Shared community spaces like the Victory Community Centre also provided key support services. One of the practitioners who worked there told me,

One of the things that I have been involved with is helping people to heat their houses. Yesterday I just gave two Chin women heaters. And ... having the Koha (donation) shed here has been a really good way for people to get access to free clothing ... The Burmese families are very comfortable coming and using that service. They feel comfortable coming and taking the food that is put out. The kai. So, I think that they've cottoned on to lots of the things that are now available in the community that helps them. They are not shy in coming forward for that sort of help.

Whilst Nelson offered a relatively wide and varied number of resettlement initiatives for a small city, responsibility for individual wellbeing not only falls upon NGOs, but also individual residents and citizens, acting in their capacity as family members, friends, neighbours, colleagues, and fellow humans. One of the resettlement practitioners reflected, 
I actually think that the Nelson community is incredibly welcoming. We see the generosity of people both in terms of their time and giving goods to people every single day. And I know that a lot of the former refugees that I have spoken to are intensely grateful for the welcome that they have had here. There are going to be individuals where that is not the case, but as a generalisation, it's fantastic what people do. I don't want to overstress it, but it really is fantastic what people do.

Māori scholars (and other individuals working with Indigenous epistemologies and methodologies) are also exploring the inclusivity of newcomers through the concept of 'manaakitanga' - whereby tangata whenua (the Indigenous people of the land) follow tikanga (local custom) and offer hospitality and care to visitors and welcome them into their whānau (family) (Buissink et al., 2017; Hazou, 2018; Hirini, 2016).

Yet, whilst many New Zealanders have reached out to volunteer for NGOs, and made an effort to acknowledge, include, and employ former refugees, encouraging individuals to care about or to take responsibility for the wellbeing of other people (and non-human entities) is not always easy, particularly in individualist societies where the self is often prioritised at the expense of the other (Fevre, 2000; Rose, 2002). Geographers Waite, Valentine, and Lewis (2014, p. 327) claimed that, "Such abstract ideals are difficult to mobilise in the context of individualisation in which the emphasis in contemporary life is on the significance of the personal rather than the social". This challenge becomes even greater when the 'other' is viewed as an 'outsider' or potential threat (Popke, 2006; Waite et al., 2014).

Some geographers have therefore shifted away from the idea of social responsibility or hospitality, to focus on processes of 'befriending' (Askins, 2016) and 'social connection', through shared interests such as painting (Kale, 2017), sports (Mathisen \& Cele, 2020), and gardening (Biglin, 2020; Rishbeth et al., 2019). However, even moving beyond responsibility to bond over shared interests can be difficult, and one of the resettlement practitioners noted, "I think a large number of the Pākehā community - the old New Zealanders or Nelsonians - have got to accept that we have a much more diverse community now. However, that doesn't mean to say that they are interested in connecting”. 
Rather than focusing on positive social connections, geographers Waite et al., (2014) suggested that maybe it is a shared recognition of the mutual capacity to hurt others, and be hurt by others that could ultimately bring people together. In this manner, people may bond over a shared feeling of suffering and injustice. Yet, this too has its challenges in individualist societies when one assumes their own actions to be justified and the actions of others to be the cause of their social ills. How to deconstruct these barriers between self and other remains an ongoing challenge; however, the current grounding of such discussions within relational, emotional everyday practice offers important potential, as geographer Askins (2016, p. 515) claimed that, "It is precisely the emotional that opens up the potential of/for making connections, and through which nuanced relationships develop, dualisms are destabilised, and meaningful encounters emerge in fragile yet hopeful ways".

\section{Self-care and being there for others}

The final point that I want to tease out from the statement above, is that some of the solutions to being and feeling well, "Lie in our own hands" (New Zealand Government, 2018a, p. 10). Individual responsibility, or self-care, is significant because participants in the He Ara Oranga report, and in the New Zealand Red Cross (2013) Refugee Health and Wellbeing Project, claimed that they wanted to be actively involved in their own recovery and everyday feelings of wellness. A desire for control over one's own health and wellbeing was also highlighted by the girls' and women's preference to keep certain matters to themselves and seek solitude when they felt upset, and by the practitioner claiming that sensory modulation had received positive feedback because it's "something that I [as an individual] can do".

For former refugees, who may have had their freedom of movement curtailed and bodies scrutinised at checkpoints and borders, and who may have been subjected to numerous invasive health-checks in refugee camps and upon arrival in their new country, taking control of one's own wellbeing may be particularly important. This desire for self-control could be even greater for women, whose physical, emotional, and mental health is often heavily controlled by the state, ethnic or religious communities, and family (through forced or denied pregnancies, abortions, or sterilisations; limited access to travel, doctors, and medication; and pressure around upholding honour and avoiding social stigma) 
(Pennington et al., 2018). By shifting away from a dependence on state or social care, former refugees can also demonstrate their independence and ability to manage their own affairs, deconstructing the notion of refugees as vulnerable, traumatised, and needy (Beaglehole, 2013; Ford, 2012).

It may also be the case that some former refugees have had trouble accessing healthcare in war-torn nations or remote villages and had to become self-reliant, or that they are used to non-Western forms of healing, making them wary or afraid of particular healthcare services. As mentioned in section 7:1, some refugee-background women felt more stressed giving birth in Nelson hospital than they did in a refugee camp due to unfamiliar sensory environments and sociocultural health-care structures, practices, and routines. This additional stress could lead to the avoidance of mainstream medical facilities. In many cultures mental health care may also be seen as irrelevant and accessing counselling services may have a stigma attached to it (Lauber \& Rossler, 2007), making it preferable to seek support elsewhere.

Taking control of one's own wellbeing is also important because lived experiences are nuanced and wellbeing is subjective. Thus, a top-down model of healthcare will not be effective for everyone, and different approaches will better suit different people (Collins \& Kearns, 2007; Conradson, 2005; Milligan \& Bingley, 2007; Tolia-Kelly, 2008). This subjectivity highlights the benefit of personalised approaches like seeking homely or therapeutic-feeling places, or adopting practices of sensory modulation, whereby the emphasis is on one's individual sense of wellbeing and the active modulation of one's environment to meet unique spatiotemporal needs. Rather than choosing between topdown or personalised care, it may be that an integration of different approaches is needed, whereby individual agency is expressed and supported, yet, institutional frameworks are also in place and accessible when needed.

In considering the responsibility of individuals to care for themselves, there is also a need to be mindful so that the negative aspects of individualism are not exacerbated (Fevre, 2000; Rose, 2002). That is to say that individuals should not exclusively focus on their own wellbeing at the expense of, or to the detriment of, others. The key factors which ultimately contributed to the girls and women feeling well in this research were their 
relationships - with family, friends, community, wildlife, the trees, mountains, and other far-off distant places. Being well thus relies upon one's investment into the wellness of others. If relationships with loved ones and society are neglected, if humans fail to nurture and care for the people and land that in return sustains them, then they ultimately undermine the foundations of their own wellbeing (Buergelt et al., 2017; Wilson, 2003).

These mutual, reciprocal practices of care and nurture were significant for the girls and women in this study. Nurturing, particularly through caring for their families and friends and tending to their gardens, was central to forming strong emotional attachments to place and feeling a sense of belonging. These attachments were formed through the joy that the women felt in creating something valuable; the time they spent familiarising themselves with the unique smells, sounds, textures, and rhythms of other people and non-human entities; and the pride that came with seeing their children, plants, and communities grow up strong and content. This being there with and for others (human or non-human) highlights an important aspect of co-becoming and becoming-well in place, which is finding meaning or purpose in one's life (Antonisch, 2010; Bawaka Country et al., 2016; hooks, 2009; Mathisen \& Cele, 2020).

In addition to asking where, when, and how people are being and becoming in place, it may therefore also be pertinent to question why they are being and becoming - what is it that, to them, makes their life worth living? When the challenge is to prevent suicide, selfharm, and feelings of depression and other mental-health related issues, then this question of meaning and purpose becomes central. Fortunately, as the girls and women have beautifully illustrated throughout this thesis, meaning can be found in all kinds of strange, wonderful, and scary places, and maybe the first step in re-approaching wellbeing is to reattune one's senses and feel the world anew, to recognise the value of those seemingly small and insignificant everyday encounters that make us who we are and make possible who we are to become.

\section{8:4 Key learnings}

In conceptualising preventative approaches to refugee mental health care, it is necessary to acknowledge that feelings of stress and anxiety often do not begin in the mind but are 
generated through interactive lived experience (with both humans and non-humans) and exacerbated by uncomfortable events and rhythms (both proximate and distant). For the girls and women in this research, their everyday multisensory experiences played a central role in their emotional relationships with, and attachments to, Nelson, and their general sense of wellbeing. From the stress of busy roads, strange-tasting foods, and foreignsounding languages, to the enchanting views of mountains and flowers and the safety of well-lit, stable houses, affective encounters with the material world shaped individual emotions, thoughts, and behaviours and vice versa. Multisensory bodies and places are thus integral to understandings of refugee mental health, and considerations of health care.

As a city, Nelson is particularly strong in its response to refugee resettlement, with a significant number of local initiatives and volunteers that assist newcomers to feel safe, happy, warm, valued, and as though they belong. However, more could be done at the state level to support and fund these initiatives, and more could be done to connect former refugees to the places that are important to them, and to think through the temporal challenges of settling into new seasonal cycles and social rhythms and routines. More could also be done to support the difficult process of navigating multiple times and places, as individuals work through nostalgic and fearful memories and live out their lives online, engaging in distant events and politics and fearing for the safety of their loved ones.

Whilst it is pivotal for residents and citizens to support one another through both positive and negative spatiotemporal processes of wellbeing, equally important is having the space and time to make one's own decisions and take control of one's own health. Rather than simply going with the flow, this means affording newcomers the freedom to also structure space and time in their own unique ways and create new harmonies through which they can feel more in-sync with their surroundings and comfortable in a dynamic and often chaotic world. This agency will require ongoing negotiations between individuals and other community members, and involve both active participation in everyday practices of wellbeing, and diligent collaboration and care. It may also rely on greater support for solitude and moments of rest, recognising the value of inactive practices of wellbeing, and the need for patience in processes of self and social transformation. 


\section{Chapter 9: Conclusion}

In this concluding chapter, I address my main research question and distil the key learnings from the previous chapters. I argue that participants' feelings of place-attachment and wellbeing were shaped through the evocation of nostalgic and fearful memories of the past, affective emotional responses in the present, and the accumulation of multisensory experiences, memories, and emotions over time. I also address the theoretical contributions that this research makes to geography and the social sciences. One such contribution is the shift from dis-placement to em-placement, which critically re-spatialises individual refugee experiences and offers a more productive and hopeful way of conceptualising wellbeing. I further enhance scholarship in geography and the social sciences through my conceptual expansion and integration of existing sensory frameworks, and my emphasis on the temporalities of place-attachment. I conclude this chapter with recommendations for future multisensory, place-attachment, and wellbeing research in geography and related disciplines, as well as for practical resettlement initiatives in Nelson.

\section{9:1 Summary of key learnings}

In many cases, refugee resettlement research has focused on the people, places, and cultures which have been lost and grieved for through forced displacement, framing placeattachment as a broken relationship; a fragmented and disconnected nostalgic longing for what was, and what could have been (Deutsch, 2005; Fried, 1966; Fullilove, 2004; Rosbrook \& Schweitzer, 2010). This focus on loss, grief, and longing is important, as displacement can be a massively upsetting event, with participants separated from their loved ones and the meaningful places where they grew up. Participants' were also separated from specific familiar experiences, like the taste of a favourite cuisine, and the reliable rhythms and routines that structured their lives and provided a sense of stability, identity, and belonging. These meaningful relationships with former places were (and remain) significant, as Elizabeth demonstrated when she said that, "If [violence] is not happening, of course, everyone like to stay [in] their own home". 
However, it is important to recognise that former refugees are not only dis-placed from their homes, but also placed elsewhere, and always in the process of interacting with new multisensory environments and making new homes as they move and resettle. To understand how interactions with strange new sights, sounds, smells, tastes, textures, and feelings might influence former refugees' sense of place-attachment and wellbeing, I aimed to explore the following question in this thesis: How do everyday multisensory experiences shape feelings of place-attachment and wellbeing for female Chin and Kayan former refugees in Nelson, Aotearoa New Zealand? Distilling the key learnings from the previous chapters, I draw out three related processes to answer this question. These processes consist of sensory memory; affective, emotional feeling; and the accumulation of sensory memories and affective feelings and emotions over time.

As demonstrated through participants' comments and paintings in Chapter Five, their everyday encounters with multisensory stimuli in Nelson often evoked nostalgic memories, enabling them to connect multiple times and places and reconstruct variations of home and personal and cultural identities in a new locale (Blunt, 2013; O'Neill and Hubbard, 2010; Sampson and Gifford, 2010). Thus, the girls and women were able to experience some semblance of normality during an otherwise tumultuous time in their lives. This sense of homeliness and familiarity was important in attracting participants to specific places in Nelson and helping them to relax, regulate their emotions, and think clearly (Akhtar, 1999; Volkan, 1999).

Yet, everyday multisensory experiences and the memories that they evoked could also be upsetting for participants, and lead to negative associations with place. Particularly troubling was that, through the act of remembering home, the girls and women's former familiar habits, routines, and rhythms now felt out-of-sync or arrhythmic with the local time-structures and pace-of-life in Nelson. Thus memories, both those consciously remembered (Damasio, 2000) and subconsciously ingrained in 'somatic modes of being' (Drozdzewski et al., 2016; Seamon, 1980), could also hinder processes of place-attachment and feelings of wellness, and pose new resettlement challenges. To address this temporal ambivalence, there is a need for resettlement researchers and practitioners to support former refugees as they mediate between past, present, and future experiences, and 
maintain and negotiate multiple place-attachments and identities (Kale et al., 2019; O'Neill \& Hubbard, 2010; Sampson \& Gifford, 2010; Tolia-Kelly, 2008).

Participants' everyday multisensory experiences in Nelson could also evoke strong affective emotional responses in the present (Damasio, 2000; Massumi, 2002; Thrift, 2008; Tuan, 1974). At times, these experiences could make the city feel overwhelming or scary to them, and lead them to avoid particular places. At other times, they could prompt in participants' feelings of contentment, enchantment, and happiness. Such experiences created 'therapeutic-feeling' places, which helped participants to relax, rejuvenate, and feel more hopeful about their future in Nelson.

To regulate their affective emotional responses, participants often sought solitude, finding private places to cry, dance, sing, pray, relax, and spend intimate moments with their loved ones or pursue other enjoyable activities. As mentioned in Chapter Six, solitude remains under-explored in place-attachment, wellbeing, and resettlement research. However, participants' desire for time-out from the city and society may reflect particular resettlement biases towards public participation and visible 'integration' by the state and local NGOs. Whilst processes of social connection and integration remain important, it is also necessary for individuals to have the time and space to rest, reflect, and re-energise in their own way, free from public scrutiny and the constant need to monitor the responses of others and their own behaviour (Burger, 1995). Through enabling ongoing feelings of safety, trustworthiness, and belonging, policy makers and practitioners can support the types of meaningful sociospatial relationships that are conducive to positive experiences of solitude (Burger, 1995; Long \& Averill, 2003), and central to a more balanced approach to wellbeing. Practices of sensory modulation may also be important in enabling individuals to have more control over their environment and further regulate their emotional responses (Champagne, 2004), as discussed in Chapter Eight.

The link between positive emotional experiences and feelings of place-attachment has been a central thread in human geography since the late 1970s and early 80s (Buttimer, 1980; Relph, 1976 Tuan, 1974; Seamon, 1980). However, in Chapter Seven I suggest caution in directly connecting positive emotions to a feeling of place-attachment, as it may be that these feelings demonstrate a fleeting attraction to place, rather than a deep sentimental 
connection. Thus, I argue that, whilst a sense of attraction may lead to a feeling of attachment, place-attachment generally occurs through the accumulation of meaningful multisensory and emotional experiences over time (Mathisen \& Cele, 2020; Wilson, 2017). Despite the potential for people-place relationships to strengthen over months and years of settlement, place-attachments cannot be taken for granted. Places and people constantly change, and thus, there is a need to understand time as open and fluid (Massey, 2005), and feelings of place-attachment as relational and emergent, always co-evolving between individuals and their sociospatial and more-than-human surroundings (Bawaka Country et al., 2016). As suggested in Chapter Three, this flow of time may not necessarily be experienced by an individual as linear, as time can also be structured and affectively felt through cycles, pauses, and moments of disorder (Jones, 2009; Marston et al., 2005; Thrift, 2008). In this manner, feelings of attraction and attachment may fluctuate with daily social rhythms or the seasons. For example, one may feel attracted to a place in spring when the flowers bloom; yet, somewhat disenchanted in the winter when the flowers disappear and the weather turns cold.

Feelings of attachment may also be influenced by distant people and places. Despite being physically present in Nelson, participants regularly engaged with imported commodities and video-called relatives and friends whom resided abroad. Through these interactions, their everyday local experiences, memories, and emotions were also shaped by transnational events, networks and flows. This politics of transnational connectivity reinforces recent geographic scholarship which has shifted away from the concept of place as bounded and self-contained, to understand place as spatially open and part of a complex geopolitical network (Amin, 2002; Massey, 2004; 2005). This openness to external influences reinforces the idea that places and place-attachments cannot be taken for granted; however, it does not diminish the importance of place and an individual desire to structure time and space in particular ways and develop a sense of belonging somewhere. 


\section{9:2 Theoretical contributions}

\section{Moving beyond dis-placement: re-spatialising individual experiences}

Whilst this thesis is specific to individual resettlement practitioners and female Chin and Kayan former refugees who were living in Nelson at the time of the research, it offers important international and interdisciplinary insights into the conceptualisation of refugee resettlement, multisensory experience, place-attachment and wellbeing. One way in which I contribute to the literature on refugee resettlement is by shifting the focus from displacement to em-placement as noted earlier. In making this shift, I do not suggest that displacement is unimportant, or that former refugees' feelings of grief, longing, and trauma should be ignored. Rather, I argue that former refugees cannot (and should not) be solely defined or understood by the root shock that forced them from their homes, or by that which is absent or missing in their lives during resettlement.

A focus on displacement inevitably delineates the spatial through the addition of the Latin prefix dis- meaning "lack of" place, "opposite of" place, "apart, asunder, in a different direction [from]” place, and in "between" places (Online Etymology Dictionary, 2020). This leaves one with the impression that refugees are deterritorialised, placeless and simply out there in 'no-man's land', unaffected by the everyday physical place-making practices of others and waiting with no horizon in sight. Yet, former refugees, like non-displaced peoples, are always present somewhere (Casey, 2009; Pink, 2015; Seamon, 2018), and the place in which they reside (be it a temporary place of asylum or a new place of settlement) can significantly impact upon their feelings, emotions, thoughts, behaviours, and relationships.

As demonstrated throughout this thesis, a shift to conceptualising former refugees, who have been resettled in a third country, as 'em-placed' over time can theoretically respatialise former refugees' experiences and offer important insights into how feelings of grief and loss may be exacerbated within everyday practice; and, critically, how these upsetting emotions may be lessened. It can also offer insights into the physical and relational generation of positive feelings and emotions, and thus pave the way for more hopeful encounters and therapeutic or enjoyable place-based experiences. In this manner, emplacement provides a tangible, productive way of exploring former refugees' 
experiences, and begins to deconstruct the notion of displaced peoples as distant others, spatially and socially lost in their new surroundings. Rather, it identifies former refugees as part of place, as relatable citizens, residents, and neighbours whom, like other residents, experience their lived realities through their senses and embodied engagements with others, human and non-human. This recognition of how diverse residents are similarly geographically placed is important in fostering empathic social connections and deconstructing negative and harmful refugee stereotypes (Askins, 2016; Noble, 2009).

Whilst a focus on emplacement (as it has evolved from the earlier focus on embodiment) is evident in geography and social science literature (Casey, 2009; Pink, 2015; Seamon, 2018), it remains relatively under-explored as a theoretical framework. Over the past decade, other researchers exploring former refugees' sensory and emotional resettlement experiences have also aimed to re-spatialise refugee experience, though few explicitly use the term 'emplacement' and more connections could be made here (Biglin, 2020; Coughlan \& Hermes, 2016; Munt, 2016; O’Neill \& Hubbard, 2010; Rishbeth et al., 2019; Sampson $\&$ Gifford, 2010).

As discussed in Chapter Three, emplacement is also useful in framing research which aims to decentralise the subjective self and focus on the relationships between people and their material surroundings - such as research exploring 'more-than-human' worlds (Whatmore, 2006), non-representational experience (McCormack, 2005; Thrift, 2008), and post-

phenomenology (Ash \& Simpson, 2016; McCormack, 2017). Chapter Four also highlights the methodological value of emplacement, as it paves the way for embodied, relational approaches to doing multisensory research (Pink, 2015) and learning from one's surroundings (Bawaka Country et al., 2016).

\section{Expanding and integrating sensory frameworks}

Another way that this research contributes to geographic and social sciences' scholarship is through my conceptual expansion and integration of existing sensory frameworks. During the research, participants often referred to philosopher Aristotle's (2015) common five senses, claiming that they enjoyed the sight of beautiful views, the sound of birdsong, the texture of soil, the smell of flowers, and the familiar tastes and flavours from home. 
However, they also discussed other sensory experiences, such as the temperature, claiming that at different times they had felt warm (Steiner, 1981), hot, cold, or freezing.

Interoceptive visceral sensations (Champagne, 2004; Hayes-Conroy \& Hayes-Conroy, 2010) were also evident through participants' memories of stomachs clenching from hunger, and their nauseating experiences of new flavours and smells from unfamiliar New Zealand foods and pesticides. Visceral experience was further alluded to through a sense of breathing (Elberfeld, 2003), with participants talking about seeking out places where they could get oxygen and fresh air, and claiming that they felt unhealthy when coughing or 'breathing in' smoke.

A sense of movement was also common (Steiner, 1981). Many of the girls and women had mentioned that they felt scared by the wind shaking their houses and overwhelmed by the busy traffic and fast pace-of-life in Nelson. In a vestibular sense, movement also entailed an awareness of one's own body moving through space when pursuing activities such as walking or dancing (Champagne, 2004). These corporal movements could lead to participants feeling tired or re-energised. Proprioceptive sensations in the muscles and joints were also linked to corporal movement, through 'hurt' ankles and difficult labourintensive work (like pulling water up from a well, gardening, or carrying children), and movement was further linked to a sense of balance, or what philosopher Steiner (1981) referred to as 'equilibrium'. For example, Mu Pli said that she felt safe in Nelson because her house felt stable, and several women mentioned feeling dizzy indoors in the summer heat.

Through a sense of breathing and movement, and mentions of 'vibrancy', participants also alluded to Steiner's (1981) sense of 'life', or being alive. His classification of speech as a sense was also pertinent, as the girls and women grappled with the pronunciation of a new language and a feeling of being outsiders due to the way that they spoke. Meanwhile, Zai Zai claimed that her 'mind was lost', echoing a sense of 'thought' or 'mind' that was common in both Eastern and Western philosophy (Bodhi, 2000; Elberfeld, 2003; Steiner, 1981). Steiner's final sense of 'ego', or self, was not explicitly discussed; however, this sense was inherent in participants' use of the reflexive pronouns ' $I$ ' and 'me' throughout 
their interviews, whereby they consciously distinguished themselves from other external physical entities.

Highlighting these multisensory experiences is important, as whilst geographers and other social scientists have often critiqued the privileging of visual knowledge in Western epistemologies (Brown, 2016; Gorman, 2017; Pink, 2015; Porteous, 1990; Relph, 1994; Rose, 1993; Trnka et al., 2013), I argue that working within the Western five-sense framework also has significant limitations, and overlooks the breadth of affective multisensory experience that influences everyday feelings, thoughts, and behaviours. In drawing upon the work of philosophers Bodhi, (2000), Elberfeld (2003), and Steiner (1981), occupational therapist Champagne (2004), geographers Hayes-Conroy and HayesConroy (2010), and anthropologists Howes (2013) and Pink (2015), I am not the first to raise concerns over the limitations of a five sense framework. However, this research provides important empirical data to support these theoretical framings. It also highlights the potential of wider sensory frameworks to contribute more holistically to refugee resettlement, a point that I expand upon later in this chapter.

My focus on the senses as integrated, or on the multisensory, also paves the way for greater exploration into other senses which may arise through a combination of the senses discussed above. For example, a sense of rhythm can emerge through sounds, sights, and movements synchronising; or a sense of arrhythmia upon sights, sounds, and movements acting in discord (Edensor, 2010). Two additional senses that participants discussed as emerging through a combination of other senses, were distance (i.e. being in close proximity to amenities, separated from loved ones, and 'connected to everything'), and time (in terms of daylight, seasons, and familiar routines). Distance could be experienced through sight, touch, sound, and temperature (or a known person or object being out-ofsight, out-of-reach, or too far away to hear or feel). Meanwhile, time could be experienced through any number of multisensory cues, such as the chirping of the cicadas, the fall of darkness, or fresh blooms of spring. These relationships between the senses are pivotal in understanding how individuals orientate themselves in, move through, and structure space and time, and thus provide a valuable approach to explore lived experience, with particular relevance for geographic research on affect (Thrift, 2008), emotional geographies (Anderson \& Smith, 2001; Davidson et al., 2005), and geographies of wellbeing (Kearns \& 
Andrews, 2010). Such integrated multisensory experiences are also important to geographies of resettlement, and questions concerning how former refugees might be affected by structural and perceptual spatiotemporal change.

\section{The importance of time-structures and rhythms in place-attachment and wellbeing}

My focus on time also offers a unique theoretical contribution to research on placeattachment and wellbeing. In existing place-attachment literature, time predominantly features in discussions around residence-time (or the length of time that an individual has lived in a locale) (Hay, 1998; Lewicka, 2011; Porteous, 1976; Relph, 1976; Steadman, 2006). With this long-term linear focus, the dynamic temporalities of night and day, changing seasons, and multiple overlapping beats, pulses, rhythms, and routines of place have been largely overlooked.

These concepts already have a strong foundation in the social sciences, particularly in geography (Edensor, 2010; Harvey, 1994; Seamon, 1980), and sociology (Lefebvre, 2004; Potts, 2015). However, I build upon these ideas by linking concepts of time and rhythm to expanded sensory frameworks, and showing how time may be affectively felt and used in nuanced ways, based upon unique individual abilities, perceptions, memories, and sociocultural knowledge. I also highlight the tendency for temporal displacement to be equally as unsettling as spatial displacement, and critically important to consider in theories of place-attachment and wellbeing for resettling refugees and other displaced individuals.

\section{9:3 Future research and resettlement recommendations}

\section{Key areas of interest}

Building upon the key theoretical learnings and contributions in this thesis, there are several areas of interest that could be expanded upon in future research. One of these areas concerns the potential difficulties that former refugees may face in adjusting to a new paceof-life. These adjustments may be particularly profound for individuals who are moving from a rural home to an urban location in New Zealand, or from a large urban metropolis to a relatively small New Zealand city. Relatively few local or international studies explore 
the emotional and psychological challenges associated with rural to urban refugee resettlement, or consider how different sized cities with variations in population density, unfamiliar expectations around timing, and unique rhythms and paces can evoke feelings of disorientation and exacerbate stress and anxiety. However, Coughlan and Hermes (2016), Gilhooly and Lee (2017), and Morse and Mudgett (2017) do touch upon these issues, and some of these ideas are being explored by $\mathrm{PhD}$ candidate Rula Y. Talahma at the University of Otago.

This rural to urban focus is important in New Zealand as the government raises its annual $\mathrm{UN}$ refugee quota and resettles more former refugees in suburban neighbourhoods (New Zealand Government, 2018b), and as diverse New Zealanders migrate out of small quiet towns and cities into large bustling urban centres like Auckland. Considerations of former refugees' prior places of residence (such as rural environments, or large mega-urban areas), and how their experiences in these places influence feelings of place-attachment and wellbeing in new locales, may also be relevant in international resettlement. In particular, a focus on how rural and urban locales and terrains are differently experienced may be important for some European nations, as they contemplate a shift to rural resettlement models (Tardis, 2019), and ways to create more green spaces in urban contexts (Barnes, 2018; Coughlan \& Hermes, 2016; Room to Heal, 2018).

There is also scope to explore how the pace-of-life or different rhythms of a city or town change through dynamic social and environmental routines, events, and seasons - and the impact of these changes on individual perceptions of, and relationships with, place. This requires a greater focus on the methodological concept of rhythmanalysis (Lefebvre, 2004; Potts, 2015) and theories of place-ballet (Seamon, 1980), place-agency (Larsen \& Johnson, 2016; McCormack, 2017), and more-than-human worlds (Abram, 1997; Whatmore, 2002). Future studies would also benefit from further research on the theories, practices, and benefits of sensory modulation in empowering individuals to cope with uncomfortable pathological stimuli and rhythms (Champagne, 2004).

Another concept that requires further attention in place-attachment, wellbeing, and refugee resettlement research, is solitude. As mentioned in Chapter Six, refugee resettlement research primarily focuses on social participation, contribution, integration and connection 
(Askins, 2016; Blunt \& Dowling, 2006; Kale et al., 2020; Rishbeth et al, 2019; Strang \& Ager, 2010). However, participants also appreciated time away from others to avoid public scrutiny and overwhelming environments, to rest and relax, take control of their surroundings, and to pursue activities that they found enjoyable or meaningful (Altman, 1975; Long \& Averill, 2003). Exploring how, when, where, why and with whom former refugees or other displaced persons are seeking solitude could offer unique insights into discussions around the value of resting and emotional renewal in wellbeing (Phipps, 2019b). It could also enhance understandings of isolation, loneliness, contentment, and creativity (Burger, 1995; Long \& Averill, 2003). Exploring the use of mobile communication technologies during times of solitude would also be interesting as individuals juggle the need for privacy and time alone with the desire to stay connected to others and visually and aurally stimulated.

\section{Disseminating multisensory research}

In addition to these theoretical explorations, there are some important methodological ideas which I was unable to execute in my research (largely due to a lack of funding) but which may inspire future multisensory projects - particularly at the exhibition or research dissemination stage. During my time at the 2018 VRN residency in Manchester, I met a musician named Ian Costabile (2019) who creates kinetic sound art and sound canvases which incorporate light and sound sensors into artworks, like paintings, to produce a unique multisensory experience. Instead of displaying various objects in different locations around the gallery and dividing viewers' attention between these objects and the artwork as was the case in our Nelson exhibition, Ian's techniques provide an opportunity for a more integrated, holistic experience. To learn from and support these types of innovative exhibition ideas, I suggest that future projects which aim to publicly disseminate participants' multisensory experiences would benefit from collaboration with professional artists (O'Neill \& Hubbard, 2010; Tolia-Kelly, 2008), sound and lighting technicians, people working with movement or kinetic sculptures, film-makers (Lobo \& Barry, 2019; Pink, 2015), musicians, chefs, and other creative individuals.

There are also companies working in special effects who could help to produce unique multisensory experiences. SensoryCo (2020) in California is a company that specialises in 
scents, water spritzer effects, weather effects including mist, rain, haze, wind, smoke and fog. They work with clients to create specific atmospheres in exhibition spaces, and on their website they claim that,

In experiential environments, atmospheric elements such as optical features, lighting, noise, and scent can be studied and/or manipulated to produce a physical response and/or cognitive impression. The implementation of atmospherics in multi-sensory spaces enhances guest or audience experiences by creating realistic environments that evoke real emotion and real reaction. Atmospheric components can impress upon customer moods, habits and perceptions. Scenting systems, in particular, have been found to affect memory, reinforce a customer's desire to buy products or services as well as influence satisfaction resulting in return visits to a venue or activity.

These types of effects could enable exhibition viewers to experience an extended range of stimuli - beyond sight, smell, sound, taste, and texture - associated with any dissemination efforts to communicate the lived experiences of former refugees to others. Such a possibility is important considering the expanded multisensory frameworks which I drew upon in this study (Bodhi, 2000; Champagne, 2004; Elberfeld, 2003; Hayes-Conroy \& Hayes-Conroy, 2010; Steiner, 1981), and the girls and women's discussions around weather, temperature, and other visceral, proprioceptive, vestibular, and interoceptive sensations. It is also important in enabling a process to foster greater empathy and understanding for long-term residents through the stimulation of affective multisensory experience.

Such effects would also make the exhibition more exciting and potentially draw in a larger crowd and more diverse population, enabling wider research dissemination. However, there are added concerns with facilitating this type of exhibition, the first being cost, and the second being a potential loss of control from participants at the exhibition stage where professionals might be likely to take over the gallery set-up (Askins \& Pain, 2011). These concerns could be remedied through the involvement of participants in all decision-making processes, though various power dynamics and ethics of representation would need to be explicitly considered and negotiated throughout the project. 


\section{Implementing and evaluating local resettlement initiatives}

Some of the conversations and ideas documented in this thesis can also be practically applied through local resettlement initiatives. Whilst making practical recommendations shifts beyond the theoretical framings of a traditional $\mathrm{PhD}$, it is an important step in participatory research which aims to be sociospatially applied and transformative (mrs kinpaisby, 2008; Pain \& Kindon, 2007). It is also a pivotal step in meeting my aim to address rising concerns over refugee mental health in Nelson and to contribute to successful resettlement outcomes.

Considering the value that the girls and women placed on connecting with urban green spaces and rural areas, a key area to focus on is strengthening former refugees' familiarity with, and access to, Nelson's rural landscapes. One resettlement practitioner suggested that the local Māori community would be well suited to facilitate these outdoor encounters due to their status as tangata whenua, and their ancestral connection to the Nelson region. As mentioned in Chapter Eight, Māori scholars are exploring the inclusion of newcomers through the concept of manaakitanga or hospitality (Buissink et al., 2017; Hazou, 2018; Hirini, 2016). Extending these ideas of hospitality into practical welcome walks around the city and surrounding terrain could be a valuable way to familiarise former refugees with the moana (ocean), and local roto (lakes), awa (rivers), maunga (mountains), and endemic flora and fauna.

In making space for Māori to share their ethnocultural and geographic knowledge and personal experiences of Whakatū (Nelson) with former refugees, both Māori as Aotearoa New Zealand's Indigenous people, as well as its ongoing colonial legacy could acknowledge that, "Refugees and other newcomers are not entering a space free from history and power" (Kale et al., 2020, p. 593). Through more bicultural engagements and welcome walks, Māori and other local Nelson residents could also become acquainted with former refugees as individual people with their own unique histories, cultures, and experiences to share, rather than as people in need of service connections. Such orientations could help to strengthen cross-cultural understanding and foster mutual recognition and respect (Askins, 2016; Noble, 2009; Wright, 2015). 
Beyond initial orientation walks, outdoor activities like fishing together could also serve to strengthen local communities, share diverse cultural knowledge and skills, and encourage more sustainable lifestyle practices. Iang Chin Sung claimed that, "No-one taught us how to fish the New Zealand way, we just practised. My husband likes fishing, so he learned and taught me too”. Little Ruth added, “I don't know how to fish here [in New Zealand]. But I know how to catch a crab. Or, I mean I know how to find a crab ...". Meanwhile, a participant in my 2015 Honours' wellbeing study said,

I was born on the West coast [in Ethiopia]. My family's life was dependent on the sea, like fishing is all they do, so I am very attached to the sea and the fish. [...] Eating fish is very important for people's bodies, health, and wellbeing [...] In terms of, like, fishing, and sea - I can't get that opportunity [in New Zealand]. Besides, the way of fishing here and back home is different ... they [Ethiopians] just use a net. I would love to show my skills and how they do it back home.

Such embodied and emotionally-significant activities could stimulate more diverse multisensory experiences for all residents of a city, and potentially transform relationships between former refugees and longer-term residents. Of particular value would be the potential for the new collective memories formed to unite individuals through their shared experiences, thereby contributing to a more inclusive and supportive place-based identity and a more capacious sense of belonging (Askins, 2016; Kale et al., 2019; O’Neill \& Hubbard, 2010; Putnam, 2007; Ratnam, 2018).

The participants in this research and I also discussed the idea of informal multisensory cooking classes to familiarise both former refugees and Nelson residents with different smells and flavours. When I asked Elizabeth if she cooked Kiwi dishes she exclaimed, "No, I don't know how! Like, you know, like BBQ, I like it, so, sometimes I grill the lamb or something. That's all I know!”. After I suggested that they and I could bring Nelson women together to teach each other how to make different cultural dishes, Elizabeth replied, "Yes! I'd really love to! You know, for a mum you have to cook nice for your family. If you don't have so many options, oh! Not good!'. Mai Aye Aye also said, "I want to selling food ... [at the] Saturday market, or any market. [There is] no Burmese food in Nelson". 
Sharing traditional recipes and food is not a new idea in Nelson. In 2012 the Nelson Multicultural Centre published a multicultural cookbook called 'Around the World in Tasty Ways'. The book included various dishes from Nelson's migrant communities as part of an initiative to improve race relations (Asia New Zealand Foundation, 2018). The New Zealand Red Cross (2020b) has produced a similar recipe book called 'Taste of Cultures'. However, reading a recipe does not foster the intimate connections and 'feelings-incommon' (Wright, 2015) that come from being in a shared multisensory space participating in an activity together. Reading may also be problematic when the recipe is translated or interpreted through a second or third language.

One practitioner, who had previously been involved in a cooking initiative in the UK called 'Cultural Kitchen', claimed that,

Food is a big one, and also the sharing and giving of food. It's having pride in it. The Cultural Kitchen concept was that each indigenous group, we'd have it on a Friday evening and they would showcase their culture and their food. So, there's people, we'd provide money for the food, they would prepare the evening and dress up in their clothing and have their music and have their food. Then they might have a little bit of a story or something, and then there were maybe some games or even dancing that they wanted to do, that was part of their culture. So, it was a fun evening focused around food and socialisation. And it became quite competitive because each group would want to do better than the Colombians, or better than whatever! But that was a whole sensory experience, colour and smell and noise and music! You know, a really sensory experience.

This type of interactive encounter is in-tune with the ideas that participants and I discussed for Nelson. However, rather than 'showcasing' culture and risking a superficial type of touristic 'cosmo-multiculturalism' (Hage, 1997, as discussed in Chapter Seven), I suggest bringing others into the creation of culture by involving them in the whole cooking process. If diverse individuals had the opportunity to collaboratively shop for, select, prepare, and cook the food as well as eat it, they could become more similarly situated and more empathically attuned to one-another's experiences (Kale et al., 2019; Pink, 2015). As the women mentioned earlier, the knowledge and skills learnt through these interactions would be invaluable in supporting their families, but they could also foster new friendships 
(Askins, 2016). Shopping together could also enable former refugees to introduce longterm residents to new shops and vice versa. Such introductions are important in fostering sociospatial familiarity and enabling individuals to "engage in the routes and mobilities of others" (O'Neill \& Hubbard, 2010, p. 50). They could also enhance social capital and the bridging of local communities to different services (Wise, 2005), and support the local economy.

Moving beyond these organised or curated cultural encounters, several practitioners also advocated for more open free-flowing spaces and events like 'Lark in the Park' where different types of people could meet one another through everyday happenings, and facilitate their own interactions, events and rhythms (Seamon, 1980). Another initiative suggested by practitioners from the Nelson Multicultural Council and Community Artworks was the building of an amphitheatre, where diverse residents could congregate and put on free shows and passers-by could stop and participate. Whilst individuals already have the opportunity to share their music at the annual Diversity Festival and other events, these cultural expressions are restricted to designated times and spaces and do not enable newcomers to interweave their unique sounds and movements into the fabric and rhythm of everyday city life, as they would be able to do with the amphitheatre.

Another way that former refugees and other Nelson residents could work together to make the city more welcoming, and to foster new friendships, is through public gardening, tree planting, and landscaping (Biglin, 2020; Rishbeth et al., 2019). These practices would enable resettling refugees from agricultural societies to share their gardening skills and feel proud that they can contribute to a healthy and sustainable lifestyle that would benefit all New Zealanders. Planting trees and gardens in public places would also enable them to physically change and contribute to the landscapes of their neighbourhoods and city, and in so doing, to see themselves 'mirrored' in their surroundings (Mathisen \& Cele, 2020).

These initiatives are particularly salient as Nelson City is currently facing a decline in activity in the $\mathrm{CBD}$ and entering a period of urban revitalisation. In an article for the Nelson Mail, local journalist Bohny (2019) wrote,

Under the new plan, the city centre development will be focused under six key objectives to attract more events to the city, develop more third spaces - places where 
people can spend time and connect with each other - and increase the liveability of the city-centre, including improving walkability and increased centre-city housing options.

The revitalisation project is currently being implemented through the City Council in collaboration with urban architect Alan Gray; however, this process of change and development provides a unique opportunity to include former refugees and other Nelson residents in the co-creation of their city. More collaborative, participatory research will be fundamental to this process, bringing together city planners, policy makers, and local communities to ensure that different voices are being heard and represented. Follow-up evaluation research will also be important to explore the long-term impact of such initiatives upon individual and collective wellbeing, social integration, and urban vitality, and to consider the transferability of these initiatives to other resettlement cities.

There also needs to be greater support for former refugees to take time out from social and urban life to rest and rejuvenate. As discussed in Chapter Six, enhancing moments of solitude is pivotal in developing a more balanced and sustainable resettlement approach whereby newcomers have the necessary time and space to process the shock of displacement and resettlement, and to express themselves freely, connect with their surroundings, and be creative. Whilst more research needs to be done in this area to understand how solitude may effectively be supported, there are several starting points which come to mind. These include greater support for childcare and driving lessons (particularly for women) to enable former refugees to take time-out from their busy schedules and/or to increase their mobility and enable their escape from the stress and faster pace of the city. Greater knowledge of how to access different places in Nelson, and familiarity with the daily rhythms and time-structures of city life may also be important, particularly in terms of knowing where and when to find places that are quiet or beautiful or conducive to a positive solitary experience.

Individuals would also benefit from greater space and resourcing to pursue meaningful solitary activities. For instance, Mu Pli enjoyed playing music during times of solitude, whilst Ruth and Mai Aye Aye enjoyed cooking. Other participants mentioned that they enjoyed sewing, but either lacked a sewing machine or the skills to sew the New Zealand 
way. Supporting these creative practices might inspire individuals to take more time to themselves and express themselves and their culture in emotionally transformative ways.

A final recommendation is to build upon the sensory modulation techniques that are already being implemented through the Stress Busters course and New Zealand district health boards. Whilst the government could provide more funding to support these existing initiatives and enable former refugees to learn how to modulate their environment and behaviour before stress and anxiety lead to depression or substance abuse, there are also ways that these techniques could be woven into everyday resettlement practice. These ways may rely on health workshops to introduce coping techniques for anxiety, as well as practitioners doing more to familiarise newcomers with simple home-modification techniques such as how to block draughts, work dehumidifiers, and safely remove mould.

As mentioned throughout this thesis, my aim is not to replace Western counselling approaches or existing mental health care services. Talking through one's problems and other forms of therapeutic intervention can be useful, and Nelson City could benefit from greater access to such services for both former refugees and other local residents. However, mental health is not only a psychological issue, it emerges through an individual's everyday embodied experiences and sociospatial and temporal relationships. By focusing on former refugees' multisensory experiences in Nelson, this thesis has demonstrated that there are multiple ways that individuals can engage with, connect to, and alter their environment to feel safe, well, and happy, and to regulate their emotions and thoughts before they begin to feel stressed, anxious, and overwhelmed. As has long been recognised by Māori and other Indigenous groups, positive emotional relationships with place are central to being and feeling well (Bawaka Country et al., 2016; Durie, 1985; Wilson, 2003), and both place and time need to be reflected in future considerations of refugee wellbeing to ensure positive resettlement outcomes. 


\section{Appendices}

Note that the research title printed on the following forms was a working title, and has since been updated.

\section{APPENDIX ONE: VUW HUMAN ETHICS APPROVAL}

TE WHARE WĀNANGa O TE ŨPOKO O TE IKA A MĀUI

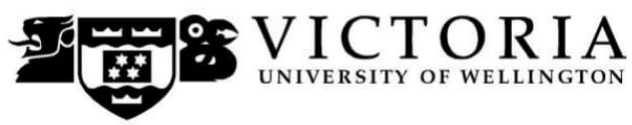

MEMORANDUM

Phone $\quad 0-4-4636028$

Email_judith.loveridge@vuw.ac.nz

\begin{tabular}{|c|c|}
\hline TO & Amber Kale \\
\hline FROM & Dr Judith Loveridge, Convenor, Human Ethics Committee \\
\hline DATE & 25 May 2018 \\
\hline PAGES & 1 \\
\hline SUBJECT & $\begin{array}{l}\text { Ethics Approval: } \mathbf{2 6 0 2 8} \\
\text { Refugee Journeys: Multisensory Processes of Place-Attachment in } \\
\text { Nelson, Aotearoa New Zealand }\end{array}$ \\
\hline
\end{tabular}

Thank you for your application for ethical approval, which has now been considered by the Human Ethics Committee.

Your application has been approved from the above date and this approval is valid for three years. If your data collection is not completed by this date you should apply to the Human Ethics Committee for an extension to this approval.

Best wishes with the research.

Kind regards

Judith Loveridge

Convenor, Victoria University Human Ethics Committee 


\section{APPENDIX TWO: PRACTITIONER RECRUITMENT LETTER}

Kia ora,

My name is Amber Kale and I am a PhD student at Victoria University, studying in the field of refugee resettlement. My research is titled, Refugee Journeys: Multisensory Processes of Place-Attachment in Nelson, Aotearoa New Zealand.

I am currently approaching Nelson refugee resettlement organisations and local community groups to ask permission to interview some of their staff members as part of my preliminary PhD research.

Each interview will last approximately one hour and include questions regarding the types of refugee resettlement services available in Nelson, and resettlement practitioner's individual experiences working with refugee-background communities, particularly the Nelson [Myanmar] community.

The purpose of these interviews is to provide background context for my main research project, which will be facilitated early next year. This project will involve working with former refugee women from the Nelson [Myanmar] community to explore how different sights, sounds, smells, tastes, textures and feelings can make people feel displaced in, or attached to, different places in Nelson. Place-attachment is important as it can help people to feel like they belong somewhere and improve their emotional wellbeing.

If your organisation is happy to contribute to this research, can the manager or director please contact me via e-mail to arrange a meeting to sign a consent form and select participants to be interviewed. I will be in Nelson for the next week or two. Alternatively, we can sign the consent form and correspond via e-mail.

My e-mail address is: kaleambe@myvuw.ac.nz

Phone: [Number retracted for publication]

Thank you for your time,

Regards,

Amber Kale. 


\title{
APPENDIX THREE: PRACTITIONER INFORMATION AND CONSENT FORMS
}

\section{Organisation information sheet and consent form}

\author{
TE WHARE WÁNANGA O TE ŪPOKO O TE IKA A MĀU \\ 59: \\ $\sim$ \\ Refugee Journeys: Multisensory Processes of Place-Attachment in Nelson, \\ Aotearoa New Zealand
}

\section{Organisation Information Sheet}

\section{Research Aim}

My name is Amber Kale and I am a PhD student at Victoria University, studying refugee resettlement within the discipline of Human Geography. My research is titled, Refugee Journeys: Multisensory Processes of Place-Attachment in Nelson, Aotearoa New Zealand. The aim of this research is to explore how different sights, sounds, smells, tastes, textures and feelings can make people feel displaced in, or attached to, different places in Nelson. Place-attachment is important as it may help people to feel like they belong somewhere and improve their emotional wellbeing. This research project has been approved by Victoria University's Human Ethics Committee, with the approval ID: 0000026028.

\section{How can your organisation help?}

I would like to invite members of your staff to participate in my research via semistructured individual interviews. Each interview will last approximately one hour and include questions regarding the types of refugee resettlement services available in Nelson, and resettlement practitioner's individual experiences working with refugeebackground communities, particularly the Nelson [Myanmar] community. Participant's interviews will be confidential and they are not obligated to share their interview transcripts with [Insert name of organisation here]. Individual names will not be used and participants will not be able to be identified in any publications. However, the name of your organisation [Insert name of organisation here] will be made public. As a representative for [insert name of organisation], you can therefore request to review academic manuscripts prior to publication.

If the staff members interviewed decide that they would like to withdraw their information, they have up until $15^{\text {st }}$ July 2018 to let me know, before I present my initial research findings at the Royal Geographical Society conference in Wales in August. Any publications resulting from this conference will be made available for use 
by [Insert name of organisation here] and the other local organisations partaking in this research.

\section{What will happen to the information shared by your staff?}

I will store a copy of each interview transcript electronically and securely for five years under the University guidelines. This storage is so that I can produce my $\mathrm{PhD}$ thesis, papers for academic conferences, and publications for academic journals. All information will be deleted at the end of five years. Any publications will be made available to your organisation for the purpose of enhancing future refugee resettlement outcomes.

Should you have any questions about the project, please do not hesitate to contact me.

\section{Contact Details:}

Researcher

Name: Amber Kale

Email: kaleambe@myvuw.ac.nz

Phone: [Number retracted for publication]

\section{Supervisor}

Name: Sara Kindon

Email: Sara.kindon@vuw.ac.nz

Phone: 044636194

Address: School of Geography Environment and Earth Sciences

Room 104, Cotton Building, Kelburn Parade

Victoria University of Wellington

PO Box 600, Wellington

Human Ethics Committee information: If you have any concerns about the ethical conduct of the research you may contact the Victoria University HEC Convener: Judith Loveridge. Email hec@vuw.ac.nz or telephone +64-4-463 6028. 


\section{TE WHARE WÃNANGA O TE OPOKO O TE IKA AMĀUI \\ 9 \\ University of wellington \\ Refugee Journeys: Multisensory Processes of Place-Attachment in Nelson, Aotearoa New Zealand}

\section{Organisation Consent Form}

I have had the opportunity to consider all the information presented to me in the information sheet and to have any questions answered to my satisfaction. I understand that my staff members may withdraw their participation at any time up to July $15^{\text {th }} 2018$. They may not withdraw after this date as the research will be shared publically at the Royal Geographical Society conference and potentially published in an academic journal.

I give my consent for staff members from [insert name of organisation] to take part in an interview.

Specifically:

- I agree that they can answer interview questions regarding refugee resettlement services in Nelson, and their experience working with refugee-background communities, particularly the Nelson [Myanmar] community.

- I understand that the researcher will keep a copy of the interview transcripts for five years to inform future publications arising from the research (listed below). I also understand that participants are not obligated to share their interview transcripts with [insert name of organisation].

- I agree that the information shared can be used for a $\mathrm{PhD}$ thesis submitted to Victoria University, for conference papers, and for publication in academic journals.

- As a representative for [insert name of organisation], I understand that I can request to review academic manuscripts prior to publication. 
This consent is given on the understanding that all information will be confidential and will not be able to be traced back to an individual source; however, the name of the organisation [Insert name of organisation here] will be made public.

\section{Contact details}

(For research communication only, these details will not be published)

Organisation representative name:

(First name) (Last name)

E-mail:

Signature

Date

/__ 


\title{
$\underline{\text { Refugee services information sheet and consent form }}$
}

\author{
TE WHARE WĀNANGA O TE ŪFOKO O TE IKA A MĀUI \\ WICTORIA \\ * $\because$ university of wellington \\ Refugee Journeys: Multisensory Processes of Place-Attachment in Nelson, \\ Aotearoa New Zealand
}

\section{Interview Information Sheet}

\section{Research Aim}

My name is Amber Kale and I am a PhD student at Victoria University, studying refugee resettlement within the discipline of Human Geography. My research is titled, Refugee Journeys: Multisensory Processes of Place-Attachment in Nelson, Aotearoa New Zealand. The aim of this research is to explore how different sights, sounds, smells, tastes, textures and feelings can make people feel displaced in, or attached to, different places in Nelson. Place-attachment is important as it may help people to feel like they belong somewhere and improve their emotional wellbeing. This research project has been approved by Victoria University's Human Ethics Committee, with the approval ID: 0000026028.

\section{How can you help?}

I invite you to participate in my research via a semi-structured individual interview. The interview will last approximately one hour and include questions regarding refugee resettlement services in Nelson, and your experience working with refugeebackground communities, particularly the Nelson [Myanmar] community. With your permission, your responses will be audio-recorded or handwritten and typed up at a later date. Your participation is voluntary. Your name will not be used and you will not be able to be identified in any publications. However, the name of the organisation for which you are interviewing [Insert name of organisation here] will be made public. If you decide you would like to withdraw your information, you have up until $15^{\text {st }}$ July 2018 to let me know, before I present my initial research findings at the Royal Geographical Society conference in Wales in August. Any publications resulting from this conference will be made available for use by [Insert name of organisation here] and the other local organisations partaking in this research. 


\section{What will happen to the information you give?}

A copy of the interview notes will be returned to you. I will store a copy of your information electronically and securely for five years under the University guidelines. This storage is so that I can produce my PhD thesis, papers for academic conferences, and publications for academic journals. All information will be deleted at the end of five years. Any publications will be made available to you and your organisation for the purpose of enhancing future refugee resettlement outcomes.

Should you have any questions about the project, please do not hesitate to contact me.

\section{Contact Details:}

\section{Researcher}

Name: Amber Kale

Email: kaleambe@myvuw.ac.nz

Phone: [Number retracted for publication]

\section{Supervisor}

Name: Sara Kindon

Email: $\underline{\text { Sara.kindon@vuw.ac.nz }}$

Phone: 044636194

Address: School of Geography Environment and Earth Sciences

Room 104, Cotton Building, Kelburn Parade

Victoria University of Wellington

PO Box 600, Wellington

Human Ethics Committee information: If you have any concerns about the ethical conduct of the research you may contact the Victoria University HEC Convener: Judith Loveridge. Email hec@vuw.ac.nz or telephone +64-4-463 6028. 


\section{TE WHARE WÄNANGA O TE OPOKO O TE IKA A MÄUI \\ W \\ 1 University of wellington \\ Refugee Journeys: Multisensory Processes of Place-Attachment in Nelson, Aotearoa New Zealand}

\section{Interview Consent Form}

I have had the opportunity to consider all the information presented to me in the information sheet and to have any questions answered to my satisfaction.

I understand that my participation is completely voluntary and that I may withdraw my participation at any time up to July $15^{\text {th }} 2018$. I may not withdraw after this date as the research will be shared publically at the Royal Geographical Society conference and potentially published in an academic journal.

I give my consent to take part in an interview.

Specifically:

- I agree to answer interview questions regarding refugee resettlement services in Nelson, and my experience working with refugee-background communities, particularly the Nelson [Myanmar] community.

- I agree to the interview being recorded, either by tape recorder or handwritten notes, to be typed up later.

- I understand that a copy of the interview notes will be returned to me, and that the researchers copy will be kept for five years to inform future publications arising from the research (listed below). Besides myself and the researcher, no-one else will have access to my interview without my permission.

- I agree that the information shared can be used for a PhD thesis submitted to Victoria University, for conference papers, and for publication in academic journals.

This consent is given on the understanding that all information will be confidential and will not be able to be traced back to an individual source; however, the name of the organisation for which I am interviewing [Insert name of organisation here] will be made public. 


\section{Contact details}

(For research communication only, these details will not be published)

Name:

(First name)

(Last name)

E-mail:

Signature

Date_________________ 


\section{APPENDIX FOUR: PRACTITIONER INTERVIEW GUIDE}

\section{Interview Question Guides}

Note: These questions are guideline questions to prompt participants, and may not be asked if participants are already engaging in relevant conversations.

\section{Demographic Questions}

1. How long have you worked with this organisation?

2. What is your role in this organisation?

3. Do you come from a refugee background?

\section{Experiential Questions}

1. What are the main challenges that [Myanmar] refugees face when they resettle in Nelson?

2. Roughly what percentage of former refugees are suffering from emotional stress, anxiety, PTSD or other mental health problems?

a) Do you believe such issues are more prevalent amongst the [Myanmar] community than other refugee communities, or the wider Nelson community?

b) What services are available to support refugee emotional and mental health in Nelson?

3. Are there any challenges in terms of former refugees developing a 'sense of belonging' in Nelson?

a) Can you share some examples of when former refugees have claimed they feel like they do / or don't belong here ...

b) In what ways have the wider Nelson community welcomed or rejected resettling refugees?

4. What kind of initiatives does your organisation have in place to enhance a sense of belonging for refugees resettling in Nelson?

a) What kind of initiatives are available to encourage social participation and wider crosscultural integration for resettling refugees?

b) How are different private and public spaces made more accessible, familiar, or 'homely' for resettling refugees?

c) What other community-based initiatives are there to enhance a sense of belonging for refugees resettling in Nelson?

5. How important do you think 'place' is in refugee resettlement?

a) What role does climate, weather, terrain play in helping or hindering a feeling of comfort and belonging for resettling refugees? 
b) What kind of difficulties do former refugees report in adjusting to unfamiliar terrain, different agricultural practices or lifestyles, or natural events such as earthquakes?

c) Can you tell me about any cases where former refugees may have mentioned missing specific landscapes from home, or specific places such as homes, cities, places of worship, family graves, or sites of spiritual significance?

d) Have there been incidents where former [Myanmar] refugees have reported feeling displaced or 'out-of-place' in Nelson due to racism, cultural or religious differences, unfamiliar environments, sensory experiences that bring back bad memories, or events such as earthquakes?

6. How is the significance of place addressed in resettlement praxis?

a) What kind of spaces and processes are available for former refugees to reflect upon, and learn how to cope with their displacement?

b) Are there any initiatives in place that can help individuals and communities to connect with the local landscape and build new emotional attachments to specific locales in Nelson?

c) Do you think more engagement with ideas and practices concerning place-attachment would be useful in helping displaced individuals and communities to develop a sense of belonging in Nelson?

7. How often, and in what ways, do former [Myanmar] refugees stay in contact with their families and friends in Myanmar?

8. When you speak with former [Myanmar] refugees, how often do they refer to memories of Myanmar?

a) What types of memories do they share?

9. How do you think that ongoing connections to, and memories of, Myanmar may impact individual's abilities to build attachments to places in Nelson and develop a sense of belonging?

10. Is there anything else you would like to mention? 


\section{APPENDIX FIVE: PARTICIPANT RECRUITMENT FLYER}

Welcome to the creative project:

\section{A Sense of $\mathbb{N e l s o n}$}

\section{January - February 2019}
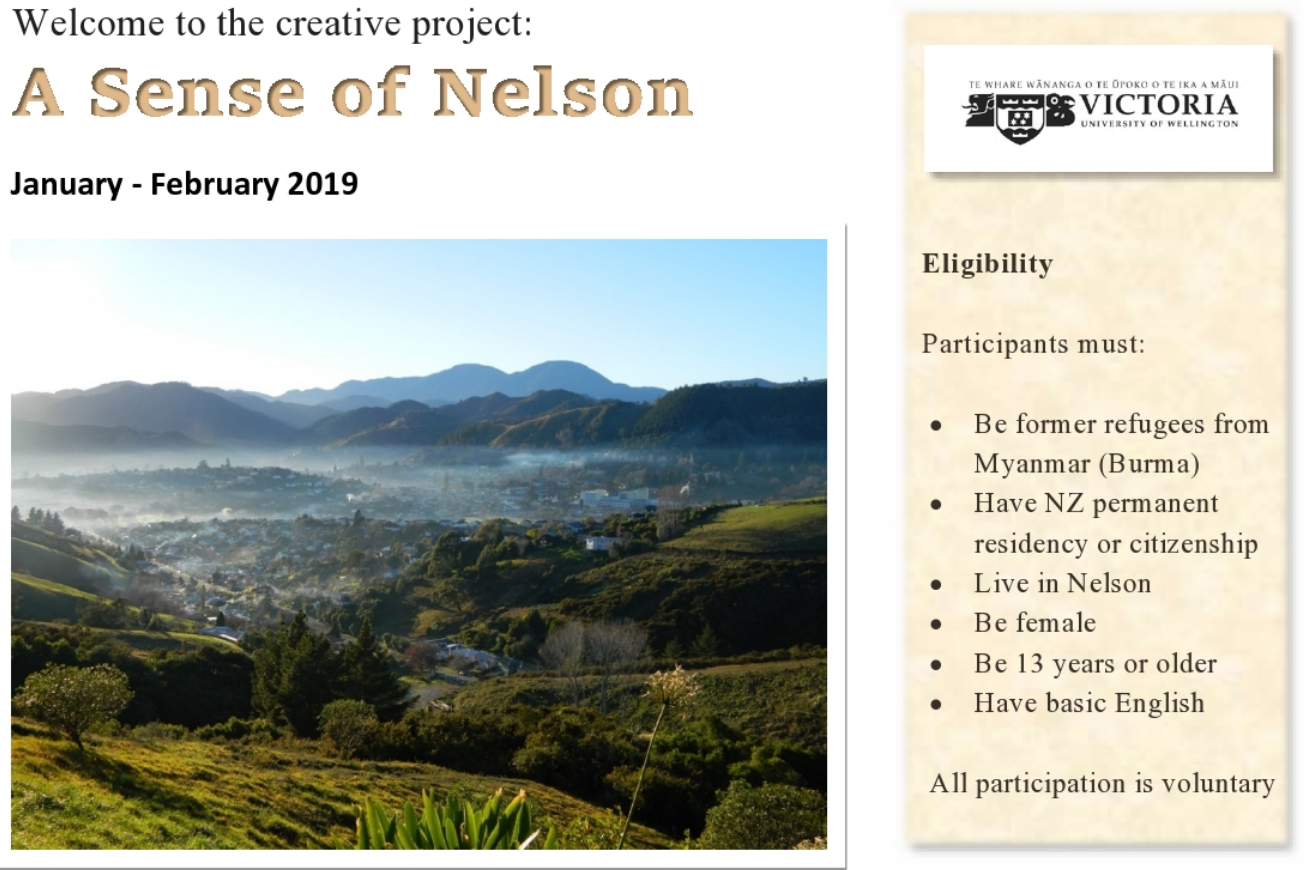

My name is Amber Kale and I am a PhD student at Victoria University doing research on refugee resettlement and wellbeing.

The aim of my research is to explore how different sights, sounds, smells, tastes, textures, and other senses make former refugees feel displaced in, or attached to, different places in Nelson City.

I am looking for 10 female participants who have come to New Zealand as refugees from Myanmar (Burma). Participation in the project will require approximately six to eight hours involvement over a period of two months, attending 1 focus group, 1 interview and field trip, 2 painting workshops, and a painting exhibition.

Please contact me for further information.

Amber Kale: [Number retracted for publication] kaleambe@myvuw.ac.nz

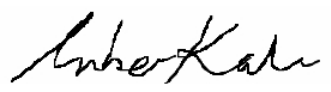

This research has been approved by the Victoria University Ethics Committee \# 0000026028 


\section{APPENDIX SIX: FIELDWORK SCHEDULE}

\begin{tabular}{|c|c|c|c|}
\hline Time /Date & Activity & Place & $\begin{array}{l}\text { Number of } \\
\text { participants }\end{array}$ \\
\hline June 2018 & Practitioner interviews & Nelson City & (11 total) \\
\hline $\begin{array}{l}\text { Wednesday } \\
13^{\text {th }} \\
10-11 \text { am }\end{array}$ & $\begin{array}{l}\text { Consent \& confidentiality forms / } \\
\text { Interview with practitioner from } \\
\text { English Language Partners }\end{array}$ & Office, CBD & 1 \\
\hline $\begin{array}{l}\text { Thursday } 14^{\text {th }} \\
11-12 \text { am }\end{array}$ & $\begin{array}{l}\text { Consent \& confidentiality forms / } \\
\text { Interview with practitioner from } \\
\text { Victory Community Centre }\end{array}$ & Office, Victory & 1 \\
\hline $\begin{array}{l}\text { Friday } 15^{\text {th }} \\
11-12 \text { am }\end{array}$ & $\begin{array}{l}\text { Consent \& confidentiality forms / } \\
\text { Interview with practitioner from a } \\
\text { local primary school }\end{array}$ & Office, Victory & 1 \\
\hline $\begin{array}{l}\text { Wednesday } \\
20^{\text {th }} \\
1-2 \mathrm{pm}\end{array}$ & $\begin{array}{l}\text { Consent \& confidentiality forms / } \\
\text { Interview with practitioners from } \\
\text { Nelson Multicultural Centre }\end{array}$ & Office, CBD & 2 \\
\hline $\begin{array}{l}\text { Thursday } 21^{\text {st }} \\
1-2 \mathrm{pm}\end{array}$ & $\begin{array}{l}\text { Consent \& confidentiality forms / } \\
\text { Interview with practitioners from a } \\
\text { local health board }\end{array}$ & Office, CBD & 2 \\
\hline $\begin{array}{l}\text { Wednesday } \\
27^{\text {th }} \\
2-3 \mathrm{pm}\end{array}$ & $\begin{array}{l}\text { Consent \& confidentiality forms / } \\
\text { Interview with practitioner from a } \\
\text { local health board }\end{array}$ & Office, Tahunanui & 1 \\
\hline $\begin{array}{l}\text { Thursday } 28^{\text {th }} \\
10-11 \text { am }\end{array}$ & $\begin{array}{l}\text { Consent \& confidentiality forms / } \\
\text { Interview with practitioner from } \\
\text { Community Artworks }\end{array}$ & Office, CBD & 1 \\
\hline $1-2 \mathrm{pm}$ & $\begin{array}{l}\text { Consent \& confidentiality forms / } \\
\text { Interview with practitioner from } \\
\text { the Red Cross }\end{array}$ & Office, Tahunanui & 1 \\
\hline $2-3 \mathrm{pm}$ & $\begin{array}{l}\text { Consent \& confidentiality forms / } \\
\text { Interview with practitioner from } \\
\text { the Red Cross }\end{array}$ & Office, Tahunanui & 1 \\
\hline January 2019 & Multisensory mapping project & Nelson City & (12 total) \\
\hline $\begin{array}{l}\text { Thursday } 24^{\text {th }} \\
1-3 \mathrm{pm}\end{array}$ & $\begin{array}{l}\text { Consent \& confidentiality forms / } \\
\text { interview / mapping activities Mu } \\
\text { Pli }\end{array}$ & ANZAC Park, CBD & 1 \\
\hline $\begin{array}{l}\text { Tuesday } 29^{\text {th }} \\
3-5 \mathrm{pm}\end{array}$ & $\begin{array}{l}\text { Consent \& confidentiality forms / } \\
\text { interview / mapping activities }\end{array}$ & $\begin{array}{l}\text { Ruth \& Little Ruth's } \\
\text { living room, Stoke }\end{array}$ & 2 \\
\hline $\begin{array}{l}\text { Thursday } 31^{\text {st }} \\
3: 30-5: 30 \mathrm{pm}\end{array}$ & $\begin{array}{l}\text { Painting workshop } \\
\text { Ruth \& Little Ruth }\end{array}$ & $\begin{array}{l}\text { Art room Nayland } \\
\text { College, Stoke }\end{array}$ & 2 \\
\hline \multicolumn{4}{|l|}{$\begin{array}{l}\text { February } \\
2019\end{array}$} \\
\hline $\begin{array}{l}\text { Friday } 1^{\text {st }} \\
11: 30-1: 30 \mathrm{pm}\end{array}$ & $\begin{array}{l}\text { Painting workshop } \\
\text { Mu Pli }\end{array}$ & $\begin{array}{l}\text { Meeting room, } \\
\text { Victory Community } \\
\text { Centre, Victory }\end{array}$ & 1 \\
\hline $\begin{array}{l}\text { Thursday } 7^{\text {th }} \\
1-2 \mathrm{pm}\end{array}$ & $\begin{array}{l}\text { Focus group: Consent \& } \\
\text { confidentiality forms }\end{array}$ & $\begin{array}{l}\text { Ngai Ciang Sung's } \\
\text { living room, Victory }\end{array}$ & 9 \\
\hline $2-3 \mathrm{pm}$ & Interview / mapping activities & $\begin{array}{l}\text { Ngai Ciang Sung's } \\
\text { living room, Victory }\end{array}$ & 1 \\
\hline
\end{tabular}




\begin{tabular}{|c|c|c|c|}
\hline $3-4 \mathrm{pm}$ & Interview / mapping activities & $\begin{array}{l}\text { Mai Aye Aye's } \\
\text { kitchen, Victory }\end{array}$ & 1 \\
\hline $\begin{array}{l}\text { Friday 8th } \\
11: 30-1: 30 \mathrm{pm}\end{array}$ & $\begin{array}{l}\text { Painting workshop } \\
\text { Mu Pli }\end{array}$ & $\begin{array}{l}\text { Meeting room, } \\
\text { Victory Community } \\
\text { Centre, Victory }\end{array}$ & 1 \\
\hline $\begin{array}{l}\text { Saturday } 9^{\text {th }} \\
2-3: 30 \mathrm{pm}\end{array}$ & Interview / mapping activities & $\begin{array}{l}\text { Ngun's garden, } \\
\text { Victory }\end{array}$ & 1 \\
\hline $\begin{array}{l}\text { Monday } 11^{\text {th }} \\
10-11: 30 \mathrm{am}\end{array}$ & Interview / mapping activities & $\begin{array}{l}\text { Sui Sung's garden, } \\
\text { Victory }\end{array}$ & 1 \\
\hline $11: 30-1 \mathrm{pm}$ & Interview / mapping activities & $\begin{array}{l}\text { Iang Chin Sung's } \\
\text { kitchen, Victory }\end{array}$ & 1 \\
\hline $\begin{array}{l}\text { Tuesday } 12^{\text {th }} \\
10-11: 30 \mathrm{am}\end{array}$ & Interview / mapping activities & $\begin{array}{l}\text { Elizabeth's garden, } \\
\text { Victory }\end{array}$ & 1 \\
\hline $\begin{array}{l}\text { Wednesday } \\
13^{\text {th }} \\
10-11: 30 \mathrm{am}\end{array}$ & $\begin{array}{l}\text { Interview / mapping activities } \\
\text { Zai Zai }\end{array}$ & Broads Field, Victory & 1 \\
\hline $11: 30-1 \mathrm{pm}$ & Interview / mapping activities & $\begin{array}{l}\text { Jenavi's garden, } \\
\text { Victory }\end{array}$ & 1 \\
\hline $1 \mathrm{pm}-2: 30 \mathrm{pm}$ & Interview / mapping activities & $\begin{array}{l}\text { Sui Tin Men's } \\
\text { garden, Whakatu }\end{array}$ & 1 \\
\hline $\begin{array}{l}\text { Friday } 15^{\text {th }} \\
11: 30- \\
1: 30 \mathrm{pm}\end{array}$ & Painting workshop & $\begin{array}{l}\text { Whare, Victory } \\
\text { Community Centre, } \\
\text { Victory }\end{array}$ & 8 \\
\hline $\begin{array}{l}\text { Tuesday } 19^{\text {th }} \\
11 \mathrm{am}- \\
12: 30 \mathrm{pm}\end{array}$ & Painting workshop & $\begin{array}{l}\text { Sui Sung's living } \\
\text { room, Victory }\end{array}$ & 1 \\
\hline $\begin{array}{l}\text { Friday } 22^{\text {nd }} \\
11-12: 30 \mathrm{pm}\end{array}$ & Painting workshop & $\begin{array}{l}\text { Sui Sung's living } \\
\text { room, Victory }\end{array}$ & 1 \\
\hline \multicolumn{4}{|l|}{ March 2019} \\
\hline Monday 18th & Set up exhibition & $\begin{array}{l}\text { G-Space Gallery } \\
\text { NMIT, Nelson CBD }\end{array}$ & \\
\hline Tuesday 19th & Set up exhibition & $\begin{array}{l}\text { G-Space Gallery } \\
\text { NMIT, Nelson CBD }\end{array}$ & \\
\hline $\begin{array}{l}\text { Wednesday } \\
20^{\text {th }}\end{array}$ & Exhibition opening night & $\begin{array}{l}\text { G-Space Gallery } \\
\text { NMIT, Nelson CBD }\end{array}$ & $\begin{array}{l}\text { Open to the } \\
\text { public }\end{array}$ \\
\hline Thursday $21^{\text {st }}$ & Exhibition seminar & $\begin{array}{l}\text { G-Space Gallery } \\
\text { NMIT, Nelson CBD }\end{array}$ & $\begin{array}{l}\text { Open to the } \\
\text { public }\end{array}$ \\
\hline Thursday $28^{\text {th }}$ & Take down exhibition & & \\
\hline
\end{tabular}




\title{
APPENDIX SEVEN: PARTICIPANT INFORMATION, CONSENT, AND CONFIDENTIALITY FORMS
}

\author{
TE WHARE WĀNANGA O TE ŪPOKO O TE IKA A MĀUI

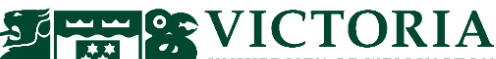 \\ Refugee Journeys: Multisensory Processes of Place-Attachment in Nelson, \\ Aotearoa New Zealand
}

\section{Participant Information Sheet}

My name is Amber Kale and I am a PhD student at Victoria University, studying refugee resettlement within the discipline of Human Geography. Welcome to my research project. Please read this information before deciding whether or not to take part. If you decide to participate, thank you. If you decide not to take part, thank you for considering my request.

\section{Research Aim}

The aim of this research is to explore how different sights, sounds, smells, tastes, textures and feelings can make people feel displaced in, or attached to, different places in Nelson. Placeattachment is important as it may help people to feel like they belong somewhere and improve their emotional wellbeing. This research project has been approved by Victoria University's Human Ethics Committee, with the approval ID: 0000026028.

\section{How can you help?}

I invite you to participate in my research via a focus group discussion, an individual interview and fieldtrip in Nelson city, and two painting workshops. This will require approximately 6 8 hours involvement over several months. Your participation is voluntary. Your names will not be used and you will not be able to be identified in any publications, unless you specifically request that your real name be used. You may withdraw from the interviews or workshops altogether without having to give reasons. If you decide you would like to withdraw your information, you have up until 30th April 2019 to let me know.

\section{Focus group}

The project will begin with a focus group to meet the other participants and share information about the research. During this time, you will create a street map showing your experiences of displacement and place-attachment in Nelson. You will then select a site from your map to visit for an individual interview with the researcher. The focus group will be approximately an hour long. 


\section{Individual interviews and field trip}

In the month following the focus group, you will meet the researcher to explore the site that you have chosen. We will walk or take public transport, depending on the distance. At the site we will engage in an activity that you have chosen, and sketch the things we see, hear, smell, taste, touch, and feel during this activity. At some stage during this trip we will have an informal interview about your experiences of displacement and place-attachment in Nelson. With your permission, your responses will be audio-recorded or handwritten and typed up at a later date. If at any point you do not want to answer a question that is fine. You may also request that the tape-recorder be turned off.

\section{Painting workshops and exhibition}

After the individual interviews, you will be invited to engage in two, two-hour-long, group workshops where you can paint your experience of place-attachment in Nelson alongside the other participants. With your permission, this painting will then be displayed as part of a public exhibition. You will also be invited to attend the exhibition on the opening night. We will decide on the location for the exhibition at a later date.

\section{What will happen to the information you give?}

A copy of the interview notes and your original artwork will be returned to you. I will store a copy of your information and artwork electronically and securely for five years under the University guidelines. This storage is so that I can produce my $\mathrm{PhD}$ thesis, papers for academic conferences, and publications for academic journals. All information will be deleted at the end of five years.

Should you have any questions about the project, please do not hesitate to contact me.

\section{Contact Details:}

Researcher

Name: Amber Kale

Email: kaleambe@myvuw.ac.nz

Phone: [Number retracted for publication]
Supervisor

Name: Sara Kindon

Email: $\underline{\text { Sara.kindon@vuw.ac.nz }}$

Phone: 044636194

Address: School of Geography Environment and Earth Sciences

Room 104, Cotton Building, Kelburn Parade

Victoria University of Wellington

PO Box 600, Wellington

Human Ethics Committee information: If you have any concerns about the ethical conduct of the research you may contact the Victoria University HEC Convener: Judith Loveridge. Email hec@vuw.ac.nz or telephone+64-4-463 6028. 


\section{TE WHARE WĀNANGA O TE UPOKO O TE IKA A MÄLI \\ S9" \\ $\sim$ \\ Refugee Journeys: Multisensory Processes of Place-Attachment in Nelson, Aotearoa New Zealand}

\section{Participant Consent to Participate}

I have had the opportunity to consider all the information presented to me in the information sheet and to have any questions answered to my satisfaction.

I understand that my participation is completely voluntary and that I may withdraw my participation at any time up to April $30^{\text {th }} 2019$. I may not withdraw after this date. I give my consent to take part in the focus group, interview field trip, and painting workshops, and for the information and images I share to be exhibited and published. Specifically:

- I agree to answer interview questions, participate in a field trip in the city, attend painting workshops, and engage in group discussions regarding my experiences of displacement and place-attachment in Nelson.

- I agree to the interviews and group discussions being recorded, either by tape recorder or handwritten notes, to be typed up later.

- I understand that a copy of the interview notes will be returned to me, and that the researchers copy will be kept for five years to inform future publications arising from the research (listed below). Besides myself and the researcher, no-one else will have access to my interview without my permission.

- I agree that the information shared, and copies of my artwork, can be used for a public art exhibition, a $\mathrm{PhD}$ thesis submitted to Victoria University, for conference papers, and for publication in academic journals.

This consent is given on the understanding that all information will be confidential and will not be able to be traced back to an individual source, unless I specifically request that my real identity be used. 
Real Name:

(First name) (Last name)

Do you want your REAL name to be used in the published research? Please circle yes, no, or first name only:

$$
\text { YES / NO / First Name Only }
$$

If you don't want your real name to be used, you can choose a different name for me to use instead and write this below. Only a first name is required. If you do not wish to use your real name or choose a different name, I will assign a pseudonym on your behalf.

\section{E-mail:}

(If you do not have e-mail access please write your address so a hardcopy of the interview notes can be sent)

Address:

\section{Signature}

Date

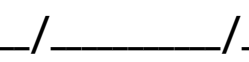




\section{te Whare Wãnanga o te úroko o te tKa a mãui VICTORIA \\ Refugee Journeys: Multisensory Processes of Place-Attachment in Nelson, Aotearoa New Zealand}

\section{Group Confidentiality Agreement}

(For participants in the Multisensory Mapping Project)

I have read the Participant Information Sheet and understand the nature of this research and its requirements for confidentiality.

I agree to maintain full confidentiality in regards to the activities that I participate in. Thus, I agree to hold in strictest confidence the identification of any individual that may be revealed during the research focus group and painting workshops in Nelson.

Name:

Signature:

Date: 


\title{
Parent information sheet and consent forms for participants under 16
}

\author{
TE WHARE WĀNANGA O TE ŪFOKO O TE IKA A MĀUI \\ 5. \\ *. 2 UNiversity OF WELlington \\ Refugee Journeys: Multisensory Processes of Place-Attachment in Nelson, \\ Aotearoa New Zealand
}

\section{Parent Information Sheet}

My name is Amber Kale and I am a PhD student at Victoria University, studying refugee resettlement within the discipline of Human Geography. Welcome to my research project. Please read this information before deciding whether or not you allow your child to take part. If you decide that they can participate, thank you. If you decide they cannot to take part, thank you for considering my request.

\section{Research Aim}

The aim of this research is to explore how different sights, sounds, smells, tastes, textures and feelings can make people feel displaced in, or attached to, different places in Nelson. Placeattachment is important as it may help people to feel like they belong somewhere and improve their emotional wellbeing. This research project has been approved by Victoria University's Human Ethics Committee, with the approval ID: 0000026028.

\section{How can your child help?}

I invite your child to participate in my research via a focus group discussion, an individual interview and fieldtrip in Nelson city, and two painting workshops. This will require approximately 6 - 8 hours involvement over two months. Their participation is voluntary. Their names will not be used and they will not be able to be identified in any publications, unless they specifically request that their real name be used. They may withdraw from the interviews or workshops altogether without having to give reasons. If they decide they would like to withdraw their information, they have up until 30 th April 2019 to let me know.

\section{Focus group}

The project will begin with a focus group to meet the other participants and share information about the research. During this time, your child will create a street map showing their experiences of displacement and place-attachment in Nelson. They will then select a site from their map to visit for an individual interview with the researcher. The focus group will be approximately an hour long.

\section{Individual interviews and field trip}

In the month following the focus group, they will meet the researcher to explore the site that they have chosen. We will walk or take public transport, depending on the distance. At the 
site we will engage in an activity that your child has chosen, and sketch the things we see, hear, smell, taste, touch, and feel during this activity. At some stage during this trip we will have an informal interview about your child's experiences of displacement and placeattachment in Nelson. With their permission, their responses will be audio-recorded or handwritten and typed up at a later date. If at any point they do not want to answer a question that is fine. They may also request that the tape-recorder be turned off.

\section{Painting workshops and exhibition}

After the individual interviews, your child will be invited to engage in two, two-hour-long, group workshops where they can paint their experience of place-attachment in Nelson alongside the other participants. With their permission, this painting will then be displayed as part of a public exhibition. You and your child will also be invited to attend the exhibition on the opening night. We will decide on the location for the exhibition at a later date.

\section{What will happen to the information your child gives?}

A copy of the interview notes and your child's original artwork will be returned to them. I will store a copy of their information and artwork electronically and securely for five years under the University guidelines. This storage is so that I can produce my PhD thesis, papers for academic conferences, and publications for academic journals. All information will be deleted at the end of five years. Should you have any questions about the project, please do not hesitate to contact me.

\section{Contact Details:}

Researcher

Name: Amber Kale

Email: kaleambe@myvuw.ac.nz

Phone: [Number retracted for publication]

\section{Supervisor}

Name: Sara Kindon

Email: Sara.kindon@vuw.ac.nz

Phone: 044636194

Address: School of Geography Environment and Earth Sciences

Room 104, Cotton Building, Kelburn Parade

Victoria University of Wellington

PO Box 600, Wellington

Human Ethics Committee information: If you have any concerns about the ethical conduct of the research you may contact the Victoria University HEC Convener: Judith Loveridge.

Email hec@vuw.ac.nz or telephone +64-4-463 6028 


\section{TE WHARE WĀNANGA O TE OPOKO O TE IKA A MÄUI \\ W 10 VICTORIA \\ aniversity of wellington \\ Refugee Journeys: Multisensory Processes of Place-Attachment in Nelson, Aotearoa New Zealand}

\section{Parental Consent for Child Participation}

I have had the opportunity to consider all the information presented to me in the information sheet and to have any questions answered to my satisfaction.

I understand that my child's participation is completely voluntary and that they may withdraw their participation at any time up to April 30 th 2019. They may not withdraw after this date.

I give my consent for my child to take part in the focus group, interview field trip, and painting workshops. Specifically:

- I agree to let my child answer interview questions, participate in a field trip in the city, attend painting workshops, and engage in group discussions regarding their experiences of displacement and place-attachment in Nelson.

- I understand that a copy of the interview notes will be returned to my child, and that the researchers copy will be kept for five years to inform future publications arising from the research (listed below). Besides my child and the researcher, no-one else will have access to their interview without their permission.

Name:

Signature

Date / 


\section{APPENDIX EIGHT: PARTICIPANT INTERVIEW QUESTIONS}

\section{Participant Demographic Questions}

1. Do you prefer the country name Myanmar, or Burma?

2. Which state or region of Myanmar / Burma did you live in?

3. Do you have New Zealand residency or citizenship?

4. Did you come to New Zealand as a quota refugee or under family reunification?

5. How long have you lived in Nelson?

6. Do you have any family living in Nelson?

7. What is your marital status?

8. Do you have children?

9. What is your ethnicity?

10. Do you identify as Burmese or \{a specific ethnic group\}?

11. Do you identify with any religious group?

12. How old are you?

13. What qualifications or skills do you have?

14. Do you work, volunteer or belong to any other social groups? 


\section{Participant Semi-Structured Individual Interview Guide}

1. Tell me about your home in Myanmar / Burma ...

a) What did the land look like? What was the weather like?

b) What type of food did people eat?

c) What kind of clothes did people wear?

2. Describe a place you felt attached to in Myanmar / Burma ...

a) What could you see, smell, hear, taste, touch, feel in this place?

b) Can you tell me about any important religious or spiritual places you would visit in Myanmar?

3. Describe a place you felt displaced in in Myanmar / Burma ...

a) What could you see, smell, hear, taste, touch, feel in this place?

4. What was the most difficult thing about leaving Myanmar?

a) What do you miss?

5. Now that you live in New Zealand, do you still think of yourself as a refugee?

a) How do you describe yourself now?

6. What did you notice when you first arrived in Nelson?

7. Describe a place in Nelson that you feel attached to ...

a) How does this place make you feel when you are there?

b) Does this place remind you of other times or places?

8. Describe a place in Nelson that you feel displaced in ...

a) How does this place make you feel when you are there?

b) Does this place remind you of other times and places?

9. Do you spend a lot of time with other [Chin / Kayan] people in Nelson? Tell me about the things you do and places you visit with these people.

a) Do you spend time with [Myanmar] people from other ethnic groups?

b) Do the different ethnic groups get along well in Nelson, despite the conflict in Myanmar / Burma?

10. Do you spend time with other people who are not from Myanmar / Burma? Tell me about the things you do and places you visit with these people.

11. Do you feel as though you belong in Nelson? Describe this sense of belonging. 

a) What could change to help you feel like you belong in Nelson?

12. Describe the different ways that you stay connected to Myanmar / Burma ...

a) How often do you talk to friends and family in Myanmar / Burma? Where do you talk to them?

b) Do you regularly stay in touch with friends and family in other countries or other parts of New Zealand?

c) When you are talking to your friends and family in other places, do you describe things that are happening around you or show them where you are through photos, snapchat, FaceTime, or Skype?

d) How often do you read news stories about what is happening in Myanmar / Burma?

e) What other ways do you stay connected to Myanmar / Burma?

13. How often do you think about, or remember things from Myanmar / Burma?

a) What are some of your favourite memories?

b) Do you ever imagine what it would be like if you still lived in Myanmar / Burma, or if you could go back there one day? How do these thoughts make you feel?

14. How does the conflict in Myanmar / Burma affect your life in Nelson?

a) How do your memories of Myanmar / Burma affect your life in Nelson?

b) Do you ever feel sad or anxious when you think about Myanmar?

c) Who do you speak to when you feel this way?

d) Are there services available in Nelson if you need help coping with sad memories?

e) Have you used these services?

15. Do you think you will ever return to live in Myanmar / Burma? Why / why not?

a) Do you consider Nelson to be your home?

b) Do you ever imagine your future in Nelson? What dreams and goals do you hope to achieve here?

16. Is there anything else you would like to say? 


\section{APPENDIX NINE: RESEARCH DISSEMINATION}

\section{Multisensory exhibition flyer}

\section{A Sense of Nelson}

\section{Welcome to the multisensory exhibition exploring place-attachment and wellbeing in refugee resettlement}

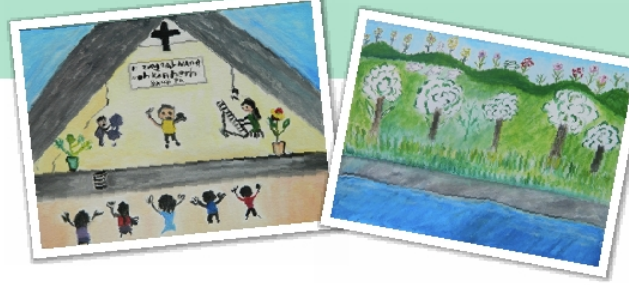

\section{Abstract}

Through everyday sights, sounds, smells, tastes, textures and other multisensory experiences, individuals familiarise themselves with the unique characteristics of their proximate environments and form meaningful emotional connections to specific landmarks, people, objects, traditions, and ways of life. These people-place relationships are often severed during forced displacement, leading many refugees to feel a sense of loss and disorientation which can negatively impact upon their health and wellbeing. As part of my Nelsonbased PhD research, I facilitated a painting project with former refugee women to explore how building new multisensory attachments to places of settlement can enable displaced individuals to regain a sense of safety and happiness during a tumultuous time in their lives. Through a series of interviews, map-making activities, and painting workshops, twelve girls and women from the Chin and Kayan communities shared their experiences of places that they felt attached to in Nelson. This exhibition showcases their paintings, offering the wider Nelson community a chance to experience our city from a different perspective and to consider how different senses and places affect their own emotional and mental health and their ability to lead happy, healthy, and productive lives. Placeattachment is not currently acknowledged as a vital part of the New Zealand refugee resettlement process; however, I argue that building emotional connections to one's surroundings is central to constructing a sense of identity and belonging in the world and enhancing individual, social, and environmental wellbeing.
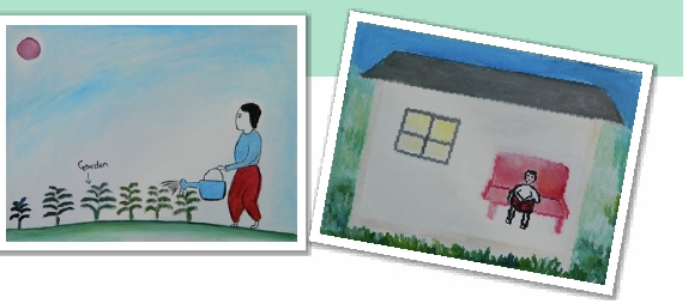

\section{Exhibition Location}

G-Space Gallery

G-Block, Nelson Marlborough Institute of

Technology, 322 Hardy Street, Nelson

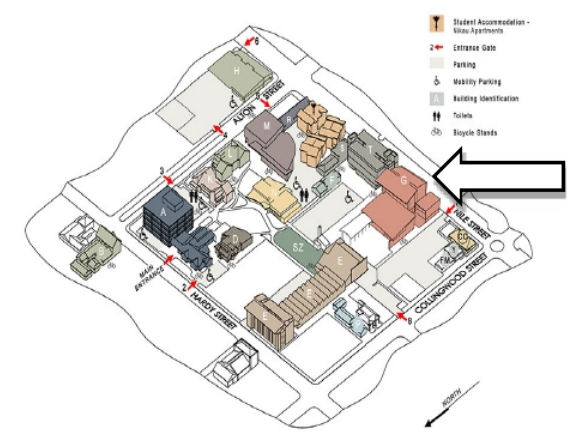

Opening Night

5:30 - 7pm Wednesday 20th March, all welcome

Exhibition Dates

The exhibition will remain on display from 8:30am

- 5:30pm from the $21^{\text {st }}-27^{\text {th }}$ March

NMIT Seminar

A seminar for NMIT students and members of the public will be run from $12: 10-1 \mathrm{pm}$ on the $21^{\text {st }}$ March at G-Space Gallery

For more information about the project contact:

Amber Kale

PhD Candidate in Human Geography at Victoria University of Wellington

Amber.Kale@vuw.ac.nz 


\section{Exhibition poster 1: Abstract and acknowledgements}

\section{A Sense of Nelson}

Welcome to the multisensory exhibition exploring place-attachment and wellbeing in refugee resettlement

\section{Abstract}

Through everyday sights, sounds, smells, tastes, textures and other multisensory experiences, individuals familiarise themselves with the unique characteristics of their proximate environments and form meaningful emotional connections to specific landmarks, people, objects, traditions, and ways of life. These people-place relationships are often severed during forced displacement, leading many refugees to feel a sense of loss and disorientation which can negatively impact upon their health and wellbeing. As part of my Nelsonbased $\mathrm{PhD}$ research, I facilitated a multisensory mapping project with former refugee women to explore how building new multisensory attachments to places of settlement can enable displaced individuals to regain a sense of safety and happiness during a tumultuous time in their lives. Through a series of interviews, map-making activities, and painting workshops, twelve girls and women from the Chin and Kayan communities shared their experiences of places that they felt attached to in Nelson. This exhibition showcases their paintings, offering the wider Nelson community a chance to experience our city from a different perspective and to consider how different senses and places affect their own emotional and mental health and their ability to lead happy, healthy, and productive lives. Placeattachment is not currently acknowledged as a vital part of the New Zealand refugee resettlement process; however, I argue that building emotional connections to one's surroundings is central to constructing a sense of identity and belonging in the world and enhancing individual, social, and environmental wellbeing.

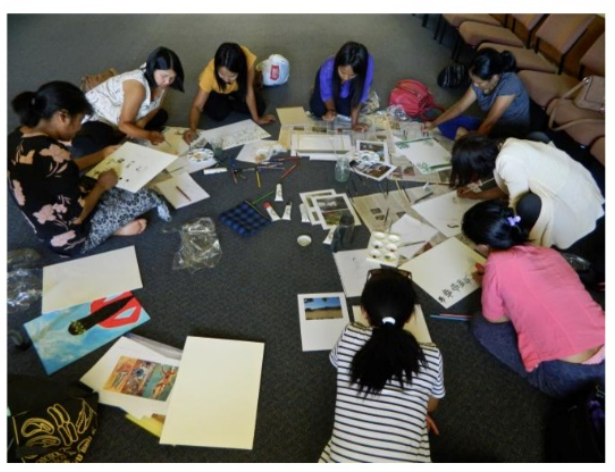

The Multisensory Mapping Project, 2019

\section{Acknowledgements}

I'd like to thank Mu Pli, Ruth, Little Ruth, Ngai Ciang Sung, Mai Aye Aye, Ngun, Sui Sung, lang Chin Sung, Elizabeth, Zai Zai, Jenavi, and Sui Tin Men for participating in the project, and all the other Nelsonians who helped me to connect with the Chin and Kayan communities. Thank you also to Nayland College and the Victory Community Centre for offering us spaces to paint, and to NMIT for providing this gallery space to exhibit our artwork. A further thank you is owed to Victoria University of Wellington (VUW) and the Women's Studies Association NZ / Pae Akoranga Wahine for funding the multisensory mapping project through a PhD scholarship and the 2018 Rosemary Seymour Research and Archives Award.

For more information about the project contact: Amber Kale, PhD Candidate in Human Geography at Victoria University of Wellington Amber.Kale@vuw.ac.nz 
Exhibition poster 2: Map of place-attachment sites in Nelson
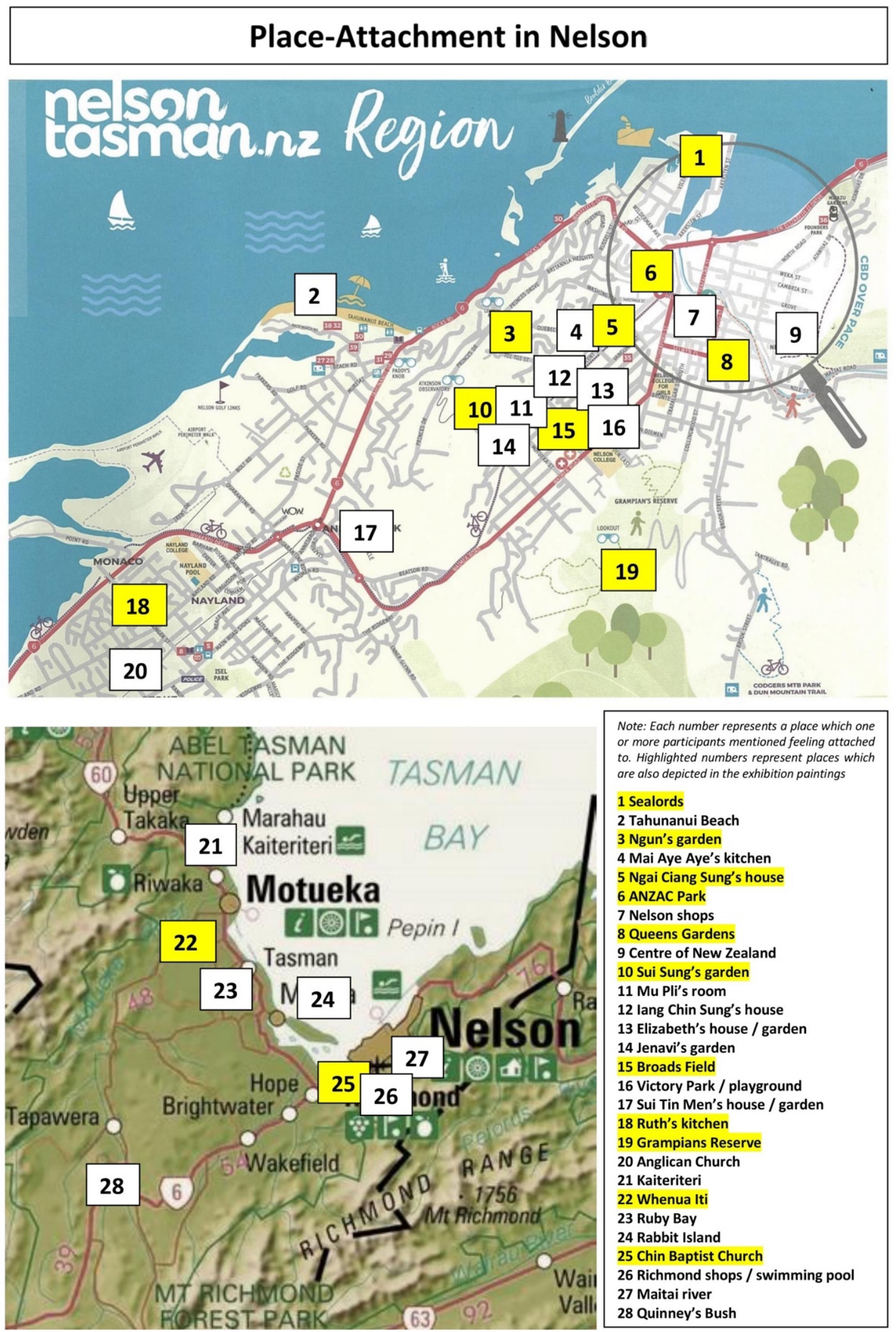

Note: Each number represents a place which one
or more participants mentioned feeling attached
to. Highlighted numbers represent places which
are also depicted in the exhibition paintings
1 Sealords
2 Tahunanui Beach
3 Ngun's garden
4 Mai Aye Aye's kitchen
5 Ngai Ciang Sung's house
6 ANZAC Park
7 Nelson shops
8 Queens Gardens
9 Centre of New Zealand
10 Sui Sung's garden
11 Mu Pli's room
12 lang Chin Sung's house
13 Elizabeth's house / garden
14 Jenavi's garden
15 Broads Field
16 Victory Park / playground
17 Sui Tin Men's house / garden
18 Ruth's kitchen
19 Grampians Reserve
20 Anglican Church
21 Kaiteriteri
22 Whenua Iti
23 Ruby Bay
24 Rabbit Island
25 Chin Baptist Church
26 Richmond shops / swimming pool
27 Maitai river
28 Quinney's Bush




\section{Exhibition poster 3: Research findings}

\section{Building Attachments to Places of Settlement}

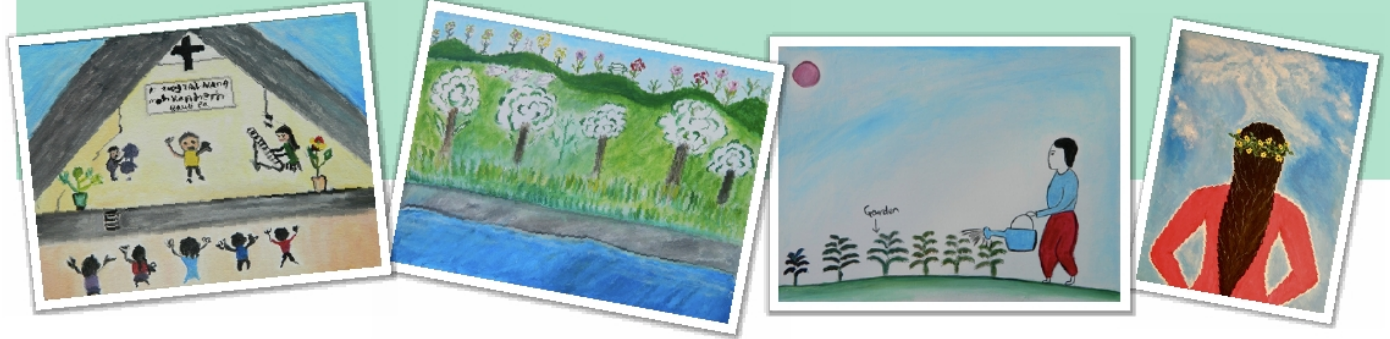

\section{Feeling safe}

During the multisensory mapping project participants mentioned feeling attached to places in Nelson where they felt safe, such as in their family homes. They appreciated their houses as they provided shelter from storms and offered electricity to meet people's basic needs and allow them to live comfortably. Several participants also mentioned feeling safe in Nelson City due to the absence of violence and warfare. Many of these safe places were connected to deeply personal past experiences where individuals had previously lived in houses without electricity that got damaged in strong winds and monsoon rains, and in regions of conflict where people were often abducted, tortured, and killed.

\section{Maintaining a connection to home}

Participants also reported feeling attached to places in Nelson which evoked positive memories of their former homes and helped them to feel connected to the places where they grew up. As all of the women came to New Zealand from mountainous regions, the hills surrounding Nelson also often evoked feelings of nostalgia and several of the women mentioned that they felt attached to places where they could look at, or go walking in, the hills. Many women from the Chin community also talked about enjoying camping out at places like Quinney's Bush, or visiting Rabbit Island to pursue the type of outdoor activities and lifestyles they were familiar with from Myanmar, and the women generally enjoyed outings to the river which reminded them of time spent with family and friends at the river back home.

\section{Places to relax, heal, and re-energise}

Other places that participants were attached to in Nelson were places where the women said that they could relax, heal, and re-energize. These were often places that evoked a feeling of peace, like quiet parks and gardens; a feeling of awe, through new experiences like seeing and smelling different flowers or feeling the ocean; or a feeling of inspiration or courage through places like Whenua Iti's outdoor course and places like churches which gave the women strength and made them feel empowered. 


\section{APPENDIX TEN: CODEBOOK SAMPLE}

\section{Section 1: Preliminary Research Scoping}

4

\begin{tabular}{|c|c|c|c|}
\hline Category & $\begin{array}{l}\text { Manifest } \\
\text { themes }\end{array}$ & Descriptive codes & Analytic codes \\
\hline $\begin{array}{l}\text { Social } \\
\text { environment }\end{array}$ & Socialisation & 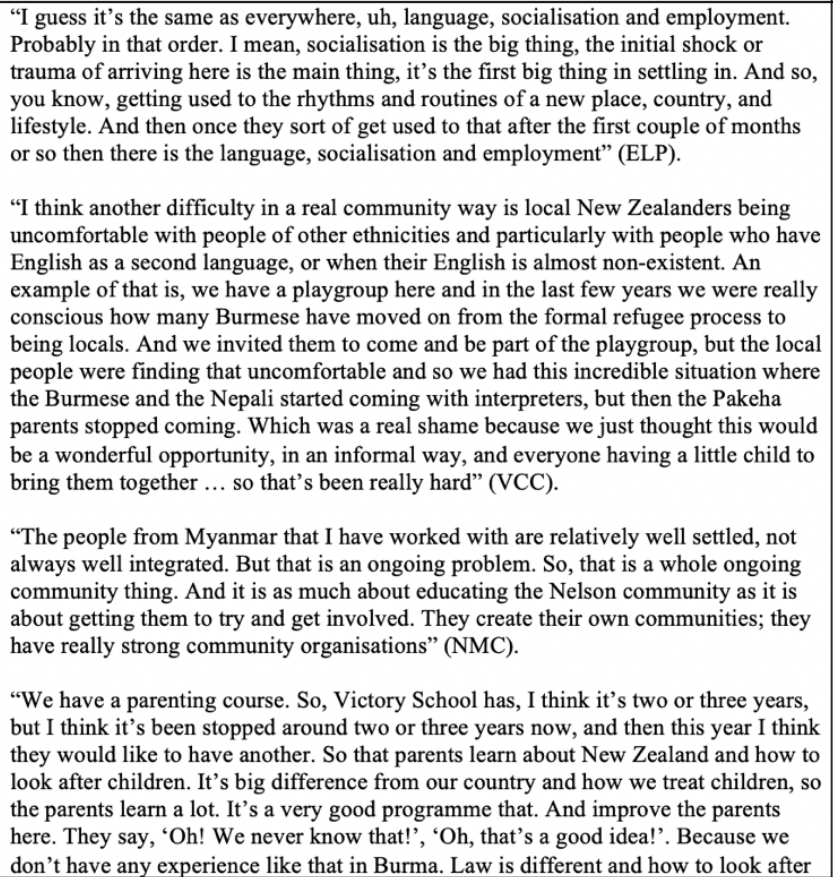 & $\begin{array}{l}\text { Othering } \\
\text { Social connection } \\
\mid \\
\begin{array}{l}\text { Social integration } \\
\text { Cross-cultural } \\
\text { education }\end{array}\end{array}$ \\
\hline
\end{tabular}

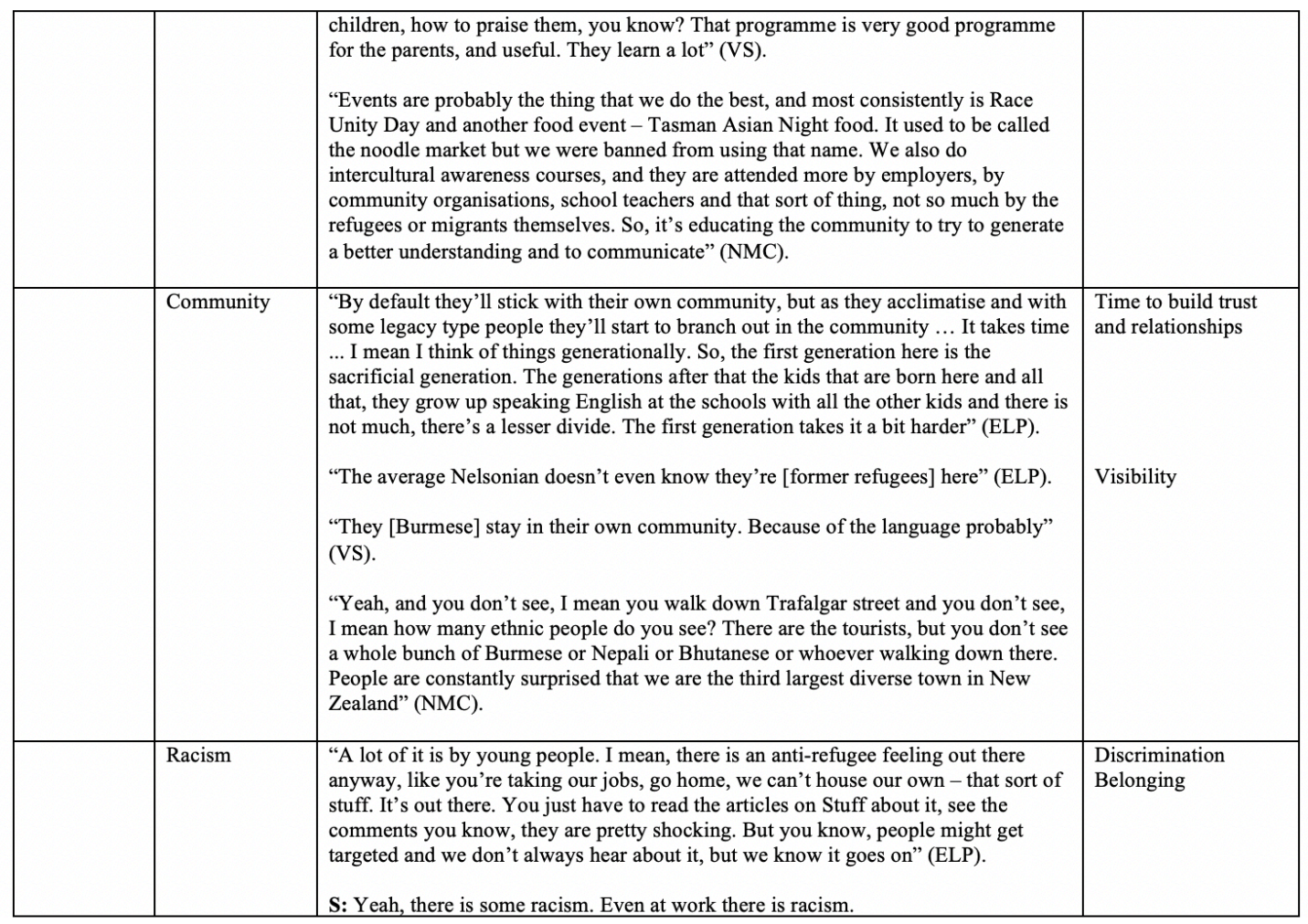




\section{Reference List}

Abram, D. (1997). The Spell of the Sensuous: Perception and Language in a More-ThanHuman World. New York: Vintage Books.

Abramovic, J., Turner, B., \& Hope, C. (2019). Entangled Recovery: Refugee Encounters in Community Gardens. The International Journal of Justice and Sustainability, 24, 696-711.

Adam, B. (1998). Timescapes of Modernity. London: Routledge.

Adey, P., Brayer, L., Masson, D., Murphy, P., Simpson, P., \& Tixier, N. (2013). 'Pour Votre Tranquillite': Ambiance, Atmosphere, and Surveillance. Geoforum, 49, 299-309.

Agyekum, B., \& Newbold, B. (2016). Religion/Spirituality, Therapeutic Landscape and Immigrant Mental Wellbeing amongst African Immigrants to Canada. Mental Health, Religion and Culture, 19(7), 674-685.

Ahmed, S. (2000). Strange Encounters: Embodied Others in Post-Coloniality. London: Routledge.

Ahmed, S. (2008). Sociable Happiness. Emotion, Space and Society, 1(1), 10-13.

Akhtar, S. (1999). The Immigrant, the Exile and the Experience of Nostalgia. Journal of Applied Psychoanalytic Studies, 2, 123-130.

Altman, I. (1975). The Environment and Social Behaviour. Monterey, California: Brooks/Cole.

Amin, A. (2002). Ethnicity and the Multicultural City: Living with Diversity. Environment and Planning A, 34, 959-98.

Amin, A. (2004). Regions Unbound: Towards a New Politics of Place. Geografiska Annaler, Series $B, 86,33-44$.

Anderson, B. (2006). Becoming and Being Hopeful: Towards a Theory of Affect. Environment and Planning D: Society and Space, 24, 733-752.

Anderson, B. (2014). Encountering Affect: Capacities, Apparatuses, Conditions. Farnham: Ashgate.

Anderson, K., \& Smith, S. (2001). Editorial: Emotional Geographies. Transactions of the Institute of British Geographers, 26(1), 7-10.

Andrews, G., Chen, S., and Myers, S. (2014). The 'Taking Place' of Health and Wellbeing: Towards Non-Representational Theory. Social Science and Medicine, 108, 210-222. 
Antonsich, M. (2010). Searching for Belonging: An Analytical Framework. Geography Compass, 4(6), 644-659.

Arev, T. (2018). Between Clothes and the Body: National and Gender Identity among Eritrean Women Refugees. Journal of Refugee Studies, 32(2), 303-321.

Aristotle. (2015). On the Heavens. London: Aeterna Press.

Ash, J. \& Simpson, P. (2016). Geography and Post-Phenomenology. Progress in Human Geography, 40(1), 48-66.

Asia New Zealand Foundation. (2018). Nelson. Retrieved from http://www.asianz.org.nz/reports/report/beyond-the-metropoles-the-asian-presence-insmall-city-new-zealand/nelson/

Askins, K. (2016). Emotional Citizenry: Everyday Geographies of Befriending, Belonging and Intercultural Encounter. Transactions of the Institute of British Geographers, 41, 515527.

Askins, K., \& Pain, R. (2011). Contact Zones: Participation, Materiality, and the Messiness of Interaction. Environment and Planning D: Society and Space, 29, 803-821.

Atkinson, S. (2013). Beyond Components of Wellbeing: The Effects of Relational and Situated Assemblage. Topoi 32(2), 137-144.

Atkinson, S., Fuller, S., \& Painter, J. (2012). Wellbeing and Place. In S. Atkinson, S. Fuller, \& J. Painter (Eds.), Wellbeing and Place (pp. 1-14). Ashgate: Farnham.

Baddeley, A., Eysenck, M., \& Anderson, M. (2009). Memory. London: Psychology Press.

Bannister, J., \& Fyfe, N. (Eds.) (2001). Fear and the City [Special issue]. Urban Studies, $38(5-6)$.

Barnes, L. G. (2018). Companion Planting Allotment Project. Retrieved from http://www.lucigorellbarnes.co.uk/companion-planting-continuing-the-allotment-project/

Barth, F., Giampieri-Deutsch, P., \& Klein, H. (2012). Sensory Perception: Mind and Matter. Wien: Springer-Verlag Wien.

Bartlet, H. (2018). Colombian Former Refugees Praise Nelson as Supportive Community. Retrieved from https://i.stuff.co.nz/nelson-mail/101514535/colombian-former- refugeespraise-nelson-as-supportive-community.

Bartos, A. (2013). Children Sensing Place. Emotion, Space and Society, 9, 89-98. 
Basso, K. H. (1996). Wisdom Sits in Places: Notes on a Western Apache Landscape. In S. Feld, \& K. H., Basso (Eds.), Senses of Place (pp. 53-90). Santa Fe, NM: School of American Research Press.

Bawaka Country, Wright, S., Suchet-Pearson, S., Lloyd, K., Burarrwanga, L., Ganambarr, R., Ganambarr-Stubbs, M., Ganambarr, B., \& Maymuru, D. (2016). Co-becoming Bawaka: Towards a Relational Understanding of Place/Space. Progress in Human Geography, 40(4), 455-475.

Beaglehole, A. (1990). Facing the Past: Looking Back at Refugee Childhood in New Zealand, 1940s -1960s. Auckland: Allen \& Unwin.

Beaglehole, A. (2013). Refuge New Zealand: A Nation's Response to Refugees and Asylum Seekers. Dunedin: Otago University Press.

Bell, S., Foley, R., Houghton, F., Maddrell, A., \& Williams, A. (2018). From Therapeutic Landscapes to Healthy Spaces, Places and Practices: A Scoping Review. Social Science \& Medicine, 196, 123-130.

Bell, S., Phoenix, C., Lovell, R., \& Wheeler, B. (2015). Seeking Everyday Wellbeing: The Coast as a Therapeutic Landscape. Social Science and Medicine, 142, 56-67.

Bennett, J. (2001). The Enchantment of Modern Life: Attachments, Crossings and Ethics. Princeton, NJ: Princeton University Press.

Biella, P. (2008). Visual Anthropology in a Time of War. In M. Strong \& L. Wilder (Eds.), Viewpoints: Visual Anthropologists at Work (pp. 141-179). Austin: University of Texas Press.

Biglin, J. (2020). Embodied and Sensory Experiences of Therapeutic Space: Refugee PlaceMaking within an Urban Allotment. Health and Place, 62, 102309.

Blunt, A. (2003). Collective Memory and Productive Nostalgia: Anglo-Indian Homemaking at McCluskieganj. Environment and Planning D: Society and Space, 21, 717-738.

Blunt, A., \& Dowling, R. (2006). Home. London: Routledge.

Bodhi, B. (2000). The Connected Discourses of the Buddha. Somerville: Wisdom Publications.

Bohny, S. (2019). Big Election Issues: How to Inject More Vitality in Nelson's CBD. Retrieved from https://www.stuff.co.nz/nelson-mail/115639294/big-election-issues-howto-inject-more-vitality-in-nelsons-cbd 
Boochani, B. (2018). No Friend but the Mountains. Sydney: Picador Australia.

Bourdieu, P. (1991). Language and Symbolic Power. Cambridge, UK: Polity Press.

Bose, P. S. (2014). Refugees in Vermont: Mobility and Acculturation in a New Immigrant Destination. Journal of Transport Geography, 36, 151-159.

Bradshaw, M., \& Stratford, E. (2010). Qualitative Research Design and Rigour. In I. Hay (Ed.), Qualitative Research Methods in Human Geography, $3^{\text {rd }}$ Edition (pp. 69-80). Ontario: Oxford University Press.

Bridge, G., \& Smith, A. (2003). Intimate Encounters: Culture-Economy-Commodity. Environment and Planning D: Society and Space, 21, 257-268.

Brown, B., Perkins, D., \& Brown, G. (2003). Place Attachment in a Revitalising Neighborhood: Individual and Block Levels of Analysis. Journal of Environmental Psychology, 23, 259-271.

Brown, K. (2016). The Haptic Pleasures of Ground-Feel: The Role of Textured Terrain in Motivating Regular Exercise. Health and Place, 46, 307-314.

Buergelt, P. T., Paton, D., Sithole, B., Sangha, K., Prasadarao, P. S. D. V., Campion, L., \& Campion, J. (2017). Living in Harmony with Our Environment: A Paradigm Shift. In D. Paton, \& D. M. Johnson (Eds.), Disaster Resilience: An Integrated Approach (2 ${ }^{\text {nd }}$ Ed) (pp. 289-307). Springfield Ill: Charles C. Thomas.

Buissink, N., Diamond, P., Hallas, J., Swann, J. \& Sciascia, A. D. (2017). Challenging a Measured University from an Indigenous Perspective: Placing 'Manaaki' at the Heart of our Professional Development Programme. Higher Education Research \& Development, 36(3), 569-582.

Bull, M., Gilroy, P., Howes, D., \& Kahn, D. (2006). Introducing Sensory Studies. The Senses and Society, 1(1), 5-7.

Burger, J. (1995). Individual Differences in Preference for Solitude. Journal of Research in Personality, 29, 85-108.

Butler, J. (1988). Performative Acts and Gender Constitution: An Essay in Phenomenology and Feminist Theory. Theatre Journal, 40(4), 519-531.

Butler, T. (2007). Memoryscape: How Audio Walks can Deepen our Sense of Place by Integrating Art, Oral History and Cultural Geography. Geography Compass, 1(3), 360372. 
Buttimer, A. (1980). Home, Reach, and the Sense of Place. In A. Buttimer \& D. Seamon (Eds.), The Human Experience of Place and Space (pp. 166-187). London: Croom Helm.

Cahill, C., Sultana, F., \& Pain, R. (2007). Participatory Ethics: Politics, Practices, Institutions. ACME: An International E-Journal for Critical Geographies, 6(3), 304- 318.

Casey, E. (1996). How to Get From Space to Place in a Fairly Short Stretch of Time. In S. Feld, \& K. Basso (Eds.), Senses of Place (pp. 13-52). Santa Fe, NM: School of American Research Press.

Casey, E. (1997). The Fate of Place. A Philosophical History. Berkeley: University of California Press.

Casey, E. (2001). Between Geography and Philosophy: What does it mean to be in the PlaceWorld? Annals of the Association of American Geographers, 91(4), 683-93.

Casey, E. (2009). Getting Back into Place, 2nd Edition. Bloomington: Indiana University Press.

Champagne, T. (2004). Sensory Approaches in Inpatient Psychiatric Settlings: Innovative Alternatives to Seclusion and Restraint. Journal of Psychological Nursing, 42(9), 35-44.

Chevalier, G., Sinatra, S., Oschman, J., Sokal, K., \& Sokal, P. (2012). Earthing: Health Implications of Reconnecting the Human Body to the Earth's Surface Electrons. Journal of Environmental and Public Health, 291541, 1-8.

Chiesura, A. (2004). The Role of Urban Parks for the Sustainability of Cities. In N. Marchettini, C. A. Brebbia, E. Tiezzi \& L. C. Wadhwa (Eds.), The Sustainable City III: Urban Regeneration and Sustainability (pp. 335-344). Southampton UK: WIT Press.

Christiansen, L., Galal, L., \& Hvenegaard-Lassen, K. (2017). Organised Cultural Encounters: Interculturality and Transformative Practices. Journal of Intercultural Studies, 38(6), 599-605.

Ciarrochi, J., Heaven, P., \& Davies, F. (2007). The Impact of Hope, Self-Esteem, and Attributional Style on Adolescents' School Grades and Emotional Well-being: A Longitudinal Study. Journal of Research in Personality 41, 1161-1178.

Classen, C. (2012). The Deepest Sense: A Cultural History of Touch. Illinois: University of Illinois Press.

Classen, C., Howes, D., \& Synott, A. (1994). Aroma: The Cultural History of Smell. London: Routledge. 
Coghlan, D. \& Brydon-Miller, M. (2014). The SAGE Encyclopaedia of Action Research. London: SAGE Publications.

Collie, P., Kindon, S., Liu, J., \& Podsiadlowski, A. (2010). Mindful Identity Negotiations: The Acculturation of Young Assyrian Women in New Zealand. International Journal of Intercultural Relations, 34, 208-220.

Collins, D., \& Kearns, R. A. (2007). Ambiguous Landscapes: Sun, Risk and Recreation on New Zealand Beaches. In A. Williams (Ed.), Therapeutic Landscapes (pp. 1532). Ashgate: Farnham.

Collins, F. L. (2008). Of Kimchi and Coffee: Globalisation, Transnationalism and Familiarity in Culinary Consumption. Social and Cultural Geography, 9(2), 151-169.

Community Action Nelson. (2019). Kids Suffer as Nelson Housing Costs Soar. Retrieved from https://www.scoop.co.nz/stories/AK1910/S00158/kids-suffer-as-nelson-housingcosts-soar.htm

Conradson, D. (2005). Landscape, Care and the Relational Self: Therapeutic Encounters in Rural England. Health \& Place, 11, 337-348.

Cooke, B., \& Kothari, U. (2001). The Case for Participation as Tyranny. In B. Cooke, \& U. Kothari (Eds.), Participation: The New Tyranny? (pp. 1-15). London: Zed Books.

Cooler, R. M. (2002). Prehistoric and Animist Periods. Illinois: Northern Illinois University.

Cope, M. (2016). Organizing and Analyzing Qualitative Data. In I. Hay (Ed.), Qualitative Research methods in Human Geography (4th ed., pp. 373-392). Ontario: Oxford University Press.

Cosgrove, D. ([1984] 1998). Social Formation and Symbolic Landscape. Wisconsin: Wisconsin University Press.

Costabile, I. (2019). Kinetic Sound Art and the Sound Canvas. In M. D. Ventura (Ed.), Proceedings of the International Conference on New Music Concepts and Inspired Education (pp. 79-84). Treviso, Italy: Academia Musicale Studio Musica.

Coughlan, R. \& Hermes, S. (2016). The Palliative Role of Green Space for Somali Bantu Women Refugees in Displacement and Resettlement, Journal of Immigrant \& Refugee Studies, 14(2), 141-155. 
Coulson, M., Oskis, A., Spencer, R., \& Gould, R. L. (2019). Tourism, Migration, and the Exodus to Virtual Worlds: Place Attachment in Massively Multiplayer Online Gamers. Psychology of Popular Media Culture. Advance online publication. http://dx.doi.org/10.1037/ppm0000244

Crang, M. (2010). Visual Methods and Methodologies. In D. Delyser, S. Herbert, S. Aitken, M. Crang, \& L. McDowell (Eds.), The Handbook of Qualitative Geography (pp. 208-225). London: Sage.

Cresswell, T. (1996). In Place/Out of Place: Geography, Ideology and Transgression. Minneapolis: University of Minnesota Press.

Cresswell, T. (2013). Geographic Thought: A Critical Introduction. New Jersey: John Wiley \& Sons.

Cytowic, R. (2003). The Man Who Tasted Shapes. Cambridge, Massachussets: MIT Press.

Damasio, A. (2000). The Feeling of What Happens: Body, Emotion and the Making of Consciousness. London: Vintage.

Darwin, C. (1859). On The Origin of Species by Means of Natural Selection. London: J. Murray.

Davidson, J., Bondi, L., \& Smith, M. (2005). Emotional Geographies. Farnham: Ashgate.

Davidson, J., \& Milligan, C. (2004). Embodying Emotion, Sensing Space: Introducing Emotional Geographies. Social and Cultural Geography, 5(4), 523-532.

DeSouza, R. (2011). Doing it for Ourselves and our Children: Refugee Women on Their Own in New Zealand. [Report]. Auckland: Refugee Services Aotearoa.

Deutsch, N. (2005). A Second Home. In B. J. Hirsch (Ed.), A Place to Call Home: AfterSchool Programs for Urban Youth (pp. 41-56). Washington, DC: American Psychological Association.

Di Fabio, A., \& Tsuda, A. (2018). The Psychology of Harmony and Harmonization: Advancing the Perspectives for the Psychology of Sustainability and Sustainable Development. Sustainability, 10, 1-15.

Dowling, R. (2016). Power, Subjectivity, and Ethics in Qualitative Research. In I. Hay (Ed.), Qualitative Research methods in Human Geography, 4th Edition (pp. 29-44). Oxford: Oxford University Press. 
Drozdzewski, D., DeNardi, S., \& Waterton, E. (2016). Geographies of Memory, Place and Identity: Intersections in Remembering War and Conflict. Geography Compass, 10(11), 447-456.

Dudley, S. (2010). Materialising Exile: Material Culture and Embodied Experience among Karenni Refugees in Thailand. New York: Berghahn Books.

Dunn, K. (2016). Interviewing. In I. Hay (Ed.), Qualitative Research Methods in Human Geography, 4th ed. (pp. 149-188). Ontario: Oxford University Press.

Durie, M. (1985). A Maori Perspective of Health. Social Science \& Medicine, 20(5), 483486.

Durie, M. (1998). Te Mana, Te Kawantaga: The Politics of Self Determination. Auckland: Oxford University Press.

Dyck, I., \& Dossa, P. (2006). Place, Health and Home: Gender and Migration in the Constitution of Healthy Space. Health and Place, 13, 691-701.

Echterhoff, G., Higgins, E., \& Levine, J. (2009). Shared Reality: Experiencing Commonality with Others' Inner States about the World. Perspectives on Psychological Science, 4(5), 496-521.

Edensor, T. (2007). Sensing the Ruin. The Senses and Society, 2(2), 217-232.

Edensor, T. (2010). Geographies of Rhythm: Nature, Place, Mobilities and Bodies. Farnham: Ashgate Publishing.

Elberfeld, R. (2003). Sensory Dimensions in Intercultural Perspective and the Problem of Modern Media and Technology. In P. Hershock, M. Stepaniants, \& R. Ames (Eds.), Technology and Cultural Values (pp. 478-490). Honolulu: University of Hawai'i Press.

Elliot, A., \& Urry, J. (2010). Mobile Lives. London: Routledge.

Elliot, S. \& Yusuf, I. (2014). 'Yes, We Can; But Together': Social Capital and Refugee Resettlement. Kötuitui: New Zealand Journal of Social Sciences Online, 9(2), 101-110.

England, M., \& Simon, S. (Eds.) (2010). Scary Cities: Urban Geographies of Fear, Difference and Belonging [Special issue]. Social and Cultural Geography, 11(3).

Evans, G. W. (2006). Child Development and the Physical Environment. Annual Review of Psychology, 57(1), 423-451.

Exploratorium. (2018). About Us. Retrieved from https://www.exploratorium.edu/about-us Feld, S. (1990). Sound and Sentiment. Philadelphia: University of Pennsylvania Press. 
Felix, E., Afifi, T., Kia-Keating, M., Brown, L., Afifi, W., \& Reyes, G. (2015). Family Functioning and Posttraumatic Growth among Parents and Youth Following Wildfire Disasters. American Journal of Orthopsychiatry, 85(2), 191-200.

Ferguson, J. (2011). Weaving a New Tartan in Scotland: The Role of Arts and Culture in Refugee Integration. West Coast Line, 44(4), 24-33.

Fevre, R.W. (2000). The Demoralisation of Western Culture. London: Continuum.

Fisher, B., \& Tronto, J. (1990). Toward a Feminist Theory of Caring. In Abel, E. and Nelson, M. (Eds.), Circles of care: work and identity in women's lives (pp. 40-62). Albany: State University of New York Press.

Fitzpatrick, F. (2002). A Search for Home: The Role of Art Therapy in Understanding the Experiences of Bosnian Refugees in Western Australia. Art Therapy, 19(4), 151- 158.

Foley, R. (2013). Small Health Pilgrimages: Place and Practice at the Holy Well. Culture and Religion, 14(1), 44-62.

Foley, R., \& Kistemann, T. (2015). Blue Space Geographies: Enabling Health in Place. Health and Place, 35, 157-165.

Ford, K. (2012). Contesting Representations of Refugee-Background Women (and Men) as 'Needy' and 'Problematic' in Healthcare Literature in Aotearoa New Zealand: Advancing the Case for a Capability-Driven Model. [Masters' Thesis]. Wellington: Victoria University of Wellington.

Franco, J., Twomey, H., Ju, K. K., Vervest, P., \& Kramer, T. (2015). The Meaning of Land in Myanmar. Amsterdam: The Transnational Institute.

Fredrickson, B. (2001). The Broaden-and-Build Theory of Positive Emotions. Philosophical Transactions of the Royal Society, 359(1449), 1367-77.

Freedom House. (2011). Worst of the Worst 2011: The World's Most Repressive Societies. [Report]. New York: Author.

Fried, M. (1966). Grieving for a Lost Home: Psychological Costs of Relocation. In J. Wilson (Ed.), Urban Renewal: The Record and the Controversy (pp. 359-379). Cambridge: The MIT Press.

Frissard, C., \& Pitts, A. (2018). The Evolution of Farming Systems and Diet in Hakha Township, Chin State, Myanmar. Yangoon: Livelihoods and Food Security Fund. 
Frydrychova, Z., Horakova, K., Vidovicova, L., \& Stepankova, H. (2017). Perception of Life Pace in the City and Countryside among Older People. The European Proceedings of Social and Cultural Behavioural Sciences, doi:10.15405/epsbs.2017.11.12.

Fullilove, M. (1997). Psychiatric Implications of Displacement: Contributions from the Psychology of Place. The American Journal of Psychiatry, 153(12), 1516-1523.

Fullilove, M. T. (2004). Root Shock: How Tearing Up City Neighbourhoods Hurts America and What We Can Do About It. New York: Ballantine Books.

Gallagher, M. \& Prior, J. (2014). Sonic Geographies: Exploring Phonographic Methods. Progress in Human Geography, 38(2), 267-84.

Gallagher, S., \& Zahavi, D. (2008). The Phenomenological Mind: An Introduction to Philosophy of Mind and Cognitive Science. London: Routledge.

Geoghegan, H., \& Woodyer, T. (2014). Cultural Geography and Enchantment: The Affirmative Constitution of Geographical Research. Journal of Cultural Geography, 31(2), 218-229.

Gesler, W. (1992). Therapeutic Landscapes: Medical Issues in the Light of the New Cultural Geography. Social Science \& Medicine, 34, 735-746.

Gesler, W. (1996). Lourdes: Healing in a Place of Pilgrimage. Health \& Place, 2, 95-105.

Gilhooly, D., \& Lee, E. (2017). Rethinking Urban Refugee Resettlement: A Case Study of One Karen Community in Rural Georgia, USA. International Migration, 55(6), 37-55.

Gilroy, P. (2006). Multiculture in Times of War: An Inaugural Lecture Given at the London School of Economics. Critical Quarterly, 48(4), 27-45.

Ginn, F., \& Demeritt, D. (2008). Nature: A Contested Concept. In N. J. Clifford (Ed.), Key Concepts in Geography (pp. 300-311). London: Sage.

Godbey, G., Graefe, A., \& James, W. (1992). The Benefits of Local Recreation and Parks Services: A Nation-Wide Study of the Perceptions of the American Public. Arlington, Va: National Recreation and Parks Association.

Gorman, R. (2017). Therapeutic Landscapes and Non-Human Animals: The Roles and Contested Positions of Animals within Care Framing Assemblages. Social and Cultural Geography, 18(3), 315-335.

Gustafson, P. (2009). Mobility and Territorial Belonging. Environment and Behavior, 41(4), 490-508. 
Gustafson, P. (2014). Place Attachment in an Age of Mobility. In L. Manzo \& P. DevineWright (Eds.), Place Attachment: Advances in Theory, Methods and Applications (pp. 3748). Abingdon: Routledge.

Hadi Curti, G. (2008). The Ghost in the City and a Landscape of Life: A Reading of Difference in Shirow and Oshii's Ghost in the Shell. Environment and Planning D: Society and Space, 26(1), 87-106.

Hage, G. (1997). At Home in the Entrails of the West: Multiculturalism, 'Ethnic Food' and Migrant Home-Building. In H. Grace, G. Hage, L. Johnson, J. Langsworth, \& M. Symonds (Eds.), Home/World: Space, Community and Marginality in Sydney's West (pp. 99-153). Annandale, NSW: Pluto Press.

Hahn, T. (2006). "It's the RUSH ... That's What Drives You to Do It": Sites of the Sensually Extreme. The Drama Review: The Journal of Performance Studies, 50(2), 87-97. Hammitt, W. (1982). Cognitive Dimensions of Wilderness Solitude. Environment and Behavior, 14(4), 478-493.

Hartig, T., \& Staats, H. (2006). The Need for Psychological Restoration as a Determinant of Environmental Preferences. Journal of Environmental Psychology, 26(3), 215-226.

Harvey, D. (1994). The Social Construction of Space and Time: A Relational Theory. Geographical Review of Japan, 67(2), 126-135.

Harvey, D. (1996). Justice, Nature and the Geography of Difference. Oxford: Blackwell.

Harvey, D. (2003). The Right to the City. International Journal of Urban and Regional Research, 27(4), 939-941.

Harvey, G. E. (1925). History of Burma: From the Earliest Times to 10 March 1824. London: Frank Cass \& Co. Ltd.

Harvey, G. (2005). Animism: Respecting the Living World. Kent Twon: Wakefield Press. Hatcher, S. (2016). Indigenous Suicide: A Global Perspective with a New Zealand Focus. The Canadian Journal of Psychiatry / La Revue Canadienne de Psychiatrie, 61(11), 684687.

Hawking, S. (2009). A Brief History of Time: From Big Bang to Black Holes. New York: Random House.

Hawkins, H., \& Straughan, E. R. (2014). Nano-Art, Dynamic Matter and the Sight/Sound of Touch. Geoforum, 51, 130-139. 
Hay, R. (1998). Sense of Place in Developmental Context. Journal of Environmental Psychology 18, 5-29.

Hayes-Conroy, A., \& Hayes-Conroy, J. (2010). Visceral Geographies, Mattering, Relating, and Defying. Geography Compass, 4(9), 1273-83.

Hazou, R. T. (2018). Performing Manaaki and New Zealand Refugee Theatre, Research in Drama Education. The Journal of Applied Theatre and Performance, 23(2), 228-241.

Health Promotion Agency. (2020). Just Ask. Just Listen. Retrieved from https://www.hpa.org.nz/campaign/just-ask-just-listen

Hicks, B., Carroll, D. Shanker, S., \& El-Zeind, A. (2017). 'Well I'm Still the Diva!' Enabling People with Dementia to Express Their Identity through Graffiti Arts: Innovative Practice. Dementia, 18(2), 814-820.

Hidalgo, C., \& Hernandez, B. (2001). Place Attachment: Conceptual and Empirical Questions. Journal of Environmental Psychology, 21, 273-281.

Hinchliffe, S., \& Whatmore, S. (2006). Living Cities: Towards a Politics of Conviviality. Science as Culture, 15, 123-138.

Hinton, D., Nickerson, A., Bryant, R. (2011). Worry, Worry Attacks, and PTSD among Cambodian Refugees: A Path Analysis Investigation. Social Science and Medicine, 72(11), $1817-1825$.

Hinton, D., Pich, V., Chhean, D., \& Pollack, M. (2004). Olfactory-Triggered Panic Attacks among Khmer Refugees: A Contextual Approach. Transcult Psychiatry, 41(2), 155-199.

Hirini, M. (2016) Tikanga Māori (Revised Edition): Living by Māori Values. Wellington: Huia.

Hooks, B. (2009). Belonging: A Culture of Place. New York: Routledge.

Howes, D. (2013). The Expanding Field of Sensory Studies. Sensory Studies, 1, 1-34.

Howes, D., \& Classen, C. (1991). Conclusion: Sounding Sensory Profiles. In D. Howes (Ed.), The Varieties of Sensory Experience: A Sourcebook in the Anthropology of the Senses (pp. 257-288). Toronto: University of Toronto press.

Howes, D., \& Classen, C. (2014). Ways of Sensing: Understanding the Senses in Society. USA: Routledge. 
Howitt, R., \& Stevens, S. (2016). Cross-Cultural Research: Ethics, Methods, and Relationships. In I. Hay (Ed.), Qualitative Research Methods in Human Geography, 4th Edition (pp. 29-44). Oxford: Oxford University Press.

Human Rights Watch. (2009). “We Are Like Forgotten People”. The Chin People of Burma:

Unsafe in Burma, Unprotected in India. New York: Author.

Human Rights Watch. (2012). World Report. New York: Author.

Husserl, E. (1989). Ideas Pertaining to a Pure Phenomenology and to a Phenomenological Philosophy, Second Book. Tr. R. Rojcewicz and A. Schuwer (Eds.). Dordrecht: Kluwer.

Hynie, M. (2018). The Social Determinants of Refugee Mental Health in the Post-Migration Context: A Critical Review. Can J Psychiatry, 63(5), 297-303.

Immigration New Zealand. (2016). Becoming a Citizen. Retrieved from http://www.immigration.govt.nz/migrant/stream/alreadyinnz/residents/becomingacitizen/ Ingold, T. (2000). The Perception of the Environment. London: Routledge.

Ingold, T. (2008). Bindings against Boundaries: Entanglements of Life in an Open World. Environment and Planning A, 40(8), 1796-1810.

International Federation of Red Cross and Red Crescent Societies. (2008). Myanmar:

Cyclone Nargis 2008 Facts and Figures. Retrieved from http://www.ifrc.org/en/news-andmedia/news-stories/asia-pacific/myanmar/myanmar-cyclone-nargis-2008-facts-andfigures/

James, B. (2010). Under the Influence: Reshaping New Zealand's Drinking Culture. [Report]. Manukau City: Salvation Army.

Javidi, H. \& Yadollahie, M. (2012). Post-Traumatic Stress Disorder. The International Journal of Occupational and Environmental Medicine, 3(1), 2-9.

Jeffrey, C. (2010). The Politics of Waiting. Retrieved from https://www.theguardian.com/commentisfree/2010/may/29/politics-waiting-social-action

Jessop, B., Brenner, N., Jones, M. (2008). Theorizing Sociospatial Relations. Environment and Planning D: Society and Space, 26, 389-401.

Jevne, R. F., \& Miller, J. E. (1999). Finding Hope: Ways to See Life in a Brighter Light. Fort Wayne, IN: Willowgreen Publishing.

Johnson, M. (2007). The Meaning of the Body: Aesthetics of Human Understanding. Chicago: University of Chicago Press. 
Jones, J. (2001). The Geography of Mental Health. Epidemiologia e Psichiatria Sociale, 10(4), 219-224.

Jones, M. (2009). Phase Space: Geography, Relational Thinking, and Beyond. Progress in Human Geography, 33, 487-506.

Jones, O. (2011). Geography, Memory and Non-Representational Geographies. Geography Compass, 5(12), 875-885.

Jones, P., \& Jam, C. (2016). Creating Ambiances, Co-constructing Place: A Poetic Transect Across the City. Area, 48(3), 317-324.

Kale, A. (2017). The Art of Social Connection: Exploring Former Refugee and Host Society Integration via a Collaborative, Participatory Painting Project in Wellington City. [Masters' Thesis]. Wellington: Victoria University of Wellington.

Kale, A. (2018). A Symbolic Representation of Wellington: How Participatory Painting Processes Enabled a More Inclusive Urban Narrative. Visual Studies, 33(4), 343-356.

Kale, A. (2019). Building Attachments to Places of Settlement: A Holistic Approach to Refugee Wellbeing in Nelson, Aotearoa New Zealand. Journal of Environmental Psychology, 65, 101315.

Kale, A., \& Kindon, S. (2020). Gender, (Im)mobility and Citizenship in a Refugee Women's Driving Programme: Exploring Emotional Citizenry in Aotearoa. Gender, Place and Culture, https://doi.org/10.1080/0966369X.2020.1846499

Kale, A., Kindon, S., \& Stupples, P. (2020). 'I am a New Zealand Citizen now - This is My Home': Refugee Citizenship and Belonging in a Postcolonising Country. Journal of Refugee Studies, 33(3), 577-598.

Kale, A., Stupples, P., \& Kindon, S. (2019). Feeling at Home: A Multisensory Analysis of Former Refugee and Host Society Residents' Integration in Wellington, Aotearoa New Zealand. Emotion, Space and Society, 33, 100615.

Kamri-McGurk, U. (2012). Resettlement Experiences of Burmese Women from Refugee Backgrounds in Wellington, Aotearoa New Zealand. [Masters' Thesis]. Wellington: Victoria University of Wellington.

Kang Pu. (2016). In My Mother's Kitchen. Retrieved from http://www.jackstraw.org/programs/ed/youth/foster.shtml 
Kaplan, S. (1978). Attention and Fascination: The Search for Cognitive Clarity. In S. Kaplan \& R. Kaplan (Eds.), Humanscape: Environments for People (pp. 84-90). Belmont CA: Duxbury.

Kaplan, R., \& Kaplan, S. (1989). The Experience of Nature: A Psychological Perspective. New York: Cambridge University Press.

Kapoor, I. (2005). Participatory Development Complicity and Desire. Third World Quarterly, 26, 1203-1220.

Kearns, R., \& Andrews, G. (2010). Geographies of Wellbeing. In S. Smith, R. Pain, S. Maston, J. Jones (Eds.), The SAGE Handbook of Social Geographies (pp. 309-328). London: Sage Publications.

Kearns, R. \& Collins, D. (2000). New Zealand Children's Health Camps: Therapeutic LandScapes Meet the Contract State. Social Science Medicine, 51, 1047-1059.

Kesby, M. (2007). Spatialising Participatory Approaches: The Contribution of Geography to a Mature Debate. Environment and Planning, 39, 2813-2831.

Kibreab, G. (2003). Citizenship Rights and Repatriation of Refugees. The International Migration Review, 37(1), 24-73.

Kidron, C. A. (2012). Breaching the Wall of Traumatic Silence: Holocaust Survivor and Descendant Person-Object Relations and the Material Transmission of the Genocidal Past. Journal of Material Culture, 17(1), 3-21.

Kindon, S. (2012). 'Thinking-through-Complicity' with Te Iwi o Ngāti Hauiti: Towards a Critical Use of Participatory Video for Research. [PhD Thesis]. Waikato: University of Waikato.

Kindon, S. (2016). Participatory Video as a Feminist Practice of Looking: 'Take Two!'. Area, 48(4), 496-503.

Kirkwood, S., Mckinlay, A., \& McVittie, C. (2013). The Mutually Constitutive Relationship between Place and Identity: The Role of Place-Identity in Discourse on Asylum Seekers and Refugees. Journal of Community \& Applied Social Psychology, 23, 453-465.

Knez, I. (2005). Attachment and Identity as Related to a Place and its Perceived Climate. Journal of Environmental Psychology, 25, 207-218.

Koch, P. (1994). Solitude: A Philosophical Encounter. Chicago: Open Court. 
Koenig, H., King, E. D., \& Carlson, B. V. (2012). Handbook of Religion and Health (2nd ed.). New York, NY: Oxford University Press.

Kogl, A. (2008). Strange Places. New York: Lexington.

Kothari, U. (2001). Power, Knowledge and Social Control in Participatory Development. In B. Cooke, \& U. Kothari (Eds.), Participation: The New Tyranny? (pp. 139-152). London: Zed Books.

Largey, G., \& Watson, D. (1972). The Sociology of Odors. American Journal of Sociology, 77(6), 1021-1034.

Larsen, S., \& Johnson, J. (2016). The Agency of Place: Toward a More-Than-Human Geographical Self. GeoHumanities, 2(1), 149-166.

Lauber, C., \& Rossler, W. (2007). Stigma Towards People with Mental Illness in Developing Countries in Asia. International Review of Psychiatry, 19(2), 157-178.

Laurier, E., \& Philo, C. (2006). Cold Shoulders and Napkins Handed: Gestures of Responsibility. Transactions of the Institute of British Geographers, 31(2), 193-207.

Law, L. (2001). Home Cooking: Filipino Women and Geographies of the Senses in Hong Kong. Ecumene, 8(3), 264-283.

Lawson, V. (2007). Geographies of Care and Responsibility. Annals of the Association of American Geographers 97, 1-11.

Lawson, V., \& Elwood, S. (2014). Encountering Poverty: Space, Class, and Poverty Politics. Antipode, 46(1), 209-228.

Lefebvre, H. (1974). The Production of Space. (Donald Nicholson-Smith, Trans, 1991). Cambridge: Blackwell.

Lefebvre, H. (2004). Rhythmanalysis: Space, Time and Everyday Life. London: Continuum.

Lenette, C., Brough, M. K., \& Cox, L. (2013). Everyday Resilience: Narratives of Single Refugee Women with Children. Qualitative Social Work, 12(5), 637-653.

Levin, R., \& Norenzayan, A. (1999). The Pace of Life in 31 Countries. Journal of CrossCultural Psychology, 30(2), 178-205.

Lewicka, M. (2011). Place Attachment: How Far Have We Come in the Last 40 Years? Journal of Environmental Psychology, 31, 207-230.

Lewis, H. (2015). Music, Dancing and Clothing as Belonging and Freedom among People Seeking Asylum in the UK. Leisure Studies, 34(1), 42-58. 
Lobo, M., \& Barry, K. (2019). Transgressing Borders with Participatory Video Technologies: Reflections on Creative Knowledge Production with Asylum Seekers in Australia. Borderlands, 18(2), 8-36.

Long, C., \& Averill, J. (2003). Solitude: An Exploration of Benefits of Being Alone. Journal for the Theory of Social Behaviour, 33(1), 21-44.

Longhurst, R., \& Johnson, L. (2014). Bodies, Gender, Place and Culture: 21 Years On. Gender, Place and Culture, 21(3), 267-278.

Longhurst, R., Johnston, L., \& Ho, E. (2009). A Visceral Approach: Cooking 'At Home' With Migrant Women in Hamilton, New Zealand. Transactions of the Institute of British Geographers, 34, 333-345.

Lonigan, C. J., Shannon, M. P., Taylor, C. M., Finch, A. J., \& Sallee, F. R. (1994). Children Exposed to Disaster: II. Risk Factors for the Development of Post-traumatic Symptomatology. Journal of the American Academy of Child and Adolescent Psychiatry, 33(1), 94-105.

Lorenz, T. (2015). Meet the 'Graffiti Grannies' of Lisbon. Retrieved from https://www.theguardian.com/artanddesign/shortcuts/2015/sep/13/the-graffiti-grannies-oflisbon

Low, K. (2005). Ruminations on Smell as a Sociocultural Phenomenon. Current Sociology, 53(3), 397-417.

Lowin, A., Hottes, J., Sandler, B., \& Bornstein, M. (1971). The Pace of Life and Sensitivity to Time in urban and Rural Settings: A Preliminary Study. The Journal of Social Psychology, 83, 247-253.

Lyon D., \& Back, L. (2012). Fishmongers in a Global Economy: Craft and Social Relations on a London Market. Sociological Research Online, 17(2), 23.

Maas, J., Verheij, R., Groenewegen, P., Vries, S., \& Spreeuwenberg, P. (2006). Green Space, Urbanity, and Health: How Strong is the Relation? Journal of Epidemiology and Community Health, 60(7), 587-592.

MacDonald, R. (2013). Music, Health, and Well-being: A Review. International Journal of Qualitative Studies on Health and Well-being, 8(1), 20635.

MacDougall, D. (2005). The Corporeal Image: Film, Ethnography, and the Senses. Princeton, NJ: Princeton University Press. 
Maddrell, A. (2013). Moving and Being Moved: More-than-Walking and Talking on Pilgrimage Walks in the Manx Landscape. Culture and Religion, 14(1), 63-77.

Malpas, J. (2001). Comparing Topologies. Philosophy and Geography, 4, 231-38.

Manzo, L. (2005). For Better or Worse: Exploring Multiple Dimensions of Place Meaning. Journal of Environmental Psychology, 25, 67-86.

Marks, L. (2000). The Skin of the Film. Durham, NC and London: Dukes University Press.

Marlowe, J. (2017). Belonging and Transnational Refugee Settlement: Unsettling the Everyday and the Extraordinary, first ed. Abingdon UK: Routledge.

Marlowe, J. (2019). Refugee Resettlement, Social Media and the Social Organization of Difference. Global Networks, 20(2), 274-291.

Marlowe, J., Bartley, A., \& Hibtit, A. (2014). The New Zealand Refugee Resettlement Strategy: Implications for Identity, Acculturation and Civic Participation. Kotuitui: New Zealand Journal of Social Sciences Online, 9(2), 60-69.

Marshall, T. (2015). Prisoners of Geography: Ten Maps that Tell You Everything You Need to Know About Global Politics. London: Elliot and Thompson Limited.

Marston, S., Jones, III, J., \& Woodward, K. (2005). Human Geography without Scale. Transactions of the Institute of British Geographers, 30, 416-432.

Martin, G., Nancarrow, S., Parker, H., Phelps, K., \& Regen, E. (2005). Place, Policy and Practitioners: On Rehabilitation, Independence and the Therapeutic Landscape in the Changing Geography of Care Provision to older People in the United Kingdom. Social Science and Medicine, 61(9), 1893-1904.

Martin, S. (2008). Justice, Women's Rights and Forced Migration. In D. Hollenbach (Ed.), Refugee Rights: Ethics, Advocacy and Africa (pp. 137-160). Washington DC: Georgetown University Press.

Massey, D. (2004). Geographies of Responsibility. Geografiska Annaler, 86B, 5-18.

Massey, D. (2005). For Space. London: Sage.

Massey, D. (2006). Landscape as a Provocation - Reflections on Moving Mountains. Journal of Material Culture, 11, 33-48.

Massumi, B. (2002). Parables for the Virtual: Movement, Affect, Sensation. Durham, North Carolina: Duke University Press. 
Mathisen, T., Cele, S. (2020). "Doing Belonging": Young Former Refugees and Their Active Engagement with Norwegian Local Communities. Fennia, 198(1), 1-18.

McBrien, J. (2014). I Oorea Te Tuaatara Ka Patu Ki Waho: Competing Priorities in the New Zealand Refugee Resettlement Strategy. Wellington: Fulbright New Zealand.

McCool, M. (2017). The 'She'll Be Right' Attitude is Killing Us. Retrieved from https://www.stuff.co.nz/national/health/98829352/the-shell-be-right-attitude-is-killing-us

McCormack, D. (2005). Diagramming Practice and Performance. Environment and Planning D: Society and Space, 23(1), 119-147.

McCormack, D. (2008). Geographies for Moving Bodies: Thinking, Dancing, Spaces. Geography Compass, 2(6), 1822-1836.

McCormack, D. (2017). The Circumstances of Post-Phenomenological Life Worlds. Transactions of the Institute of British Geographers, 42, 2-13.

McDonald, B., Spaaij, R., \& Dukic, D. (2019). Moments of Social Inclusion: Asylum Seekers, Football and Solidarity. Sport in Society, 22(6), 935-949.

McDuffee, D. (2015). Earthing: The Silliest Health Scam Ever? Retrieved from http://beyondgrowth.net/technology-of-the-self/earthing-silliest-health-scam-ever/

McGeachan, C., \& Philo, C. (2017). Occupying Space: Mental Health Geography and Global Directions. In R. White, S. Jain, D. Orr, \& U. Read. (Eds). The Palgrave Handbook of Sociocultural perspectives on Global Mental Health (pp. 31-50). London: Palgrave Macmillan.

McGuire, M. (2016). Individual Sensory Experiences, Socialized Senses, and Everyday Lived Religion in Practice. Social Compass, 63(2), 152-162.

McKenzie, R., Connor, B., \& Bodeker, G. (1999). Increased Summertime UV Radiation in New Zealand in Response to Ozone Loss. Science, 285(5434), 1709-11.

McKie, L., Gregory, S. \& Bowlby, S. (2002). Shadow Times: The Temporal and Spatial Frameworks and Experiences of Caring and Working. Sociology, 36, 897-924.

McKinsey, K. (2006). In Search of Freedom, Burmese Refugees Head for New Life in America. Retrieved from https:/www.unhcr.org/news/latest/2006/10/452f99404/searchfreedom-burmese-refugees-head-new-life-america.html

McLean, K. (2017). Sensory Maps. Retrieved from http://sensorymaps.com/about/ 
McLeod, A. \& Reeve, M. (2005). The Health Status of Quota Refugees Screened by New Zealand's Auckland Public Health Service Between 1995 and 2000. The New Zealand Medical Journal, 118(1224), 1-17.

McNeill, W. (1994). Reasserting the Polyethnic Norm. In J. Hutchinson, \& A. D. Smith (Eds.), Nationalism (pp. 300-305). Oxford: Oxford University Press.

Melemis, S., \& Tixier, N. (2010). Urban Transects ARCC/EAAE Annual Conference, Washington DC. Detrieved from http://www.aia.org/aiaucmp/groups/aia/documents/pdf/aiab087179.pdf

Merleau-Ponty, M. (1962). Phenomenology of Perception. London: Routledge and Kegan Paul Ltd.

Milligan, C., Gatrell, A., \& Bingley, A. (2004). 'Cultivating Health': Therapeutic Landscapes and Older People in Northern England. Social Science \& Medicine, 58, 17811793.

Milligan, C., \& Bingley, A. (2007). Restorative Places or Scary Spaces? The Impact of Woodland on the Mental Health of Young Adults. Health \& Place 13(4), 799-811.

Minority Rights Group International. (2018). Myanmar / Burma - Chin. Retrieved from http://minorityrights.org/minorities/chin/

Mirante, E. (2006). The Dragon Mothers Polish their Metal Coils. Retrieved from https://web.archive.org/web/20081212153427/http://www.guernicamag.com/features/229/t he_dragon_mothers/

Mon, K. H. (2014). Helping Burmese Refugees Start New Lives in New Zealand. Retrieved from https://www.irrawaddy.com/in-person/interview/helping-burmese-refugees-startnew-lives-new-zealand.html

Moore, E. (1981). A Prison Environment's Effect on 20 Health Care Service Demands. Environmental Systems, 21(11), 17-34.

Morrison, P. (2016). Pride in the City. Region, 3(2), 103-124.

Morse, C., \& Mudgett, J. (2017). Longing for Landscape: Homesickness and Place Attachment among Rural Out-Migrants in the $19^{\text {th }}$ and $21^{\text {st }}$ Centuries. Journal of Rural Studies, 50, 95-103.

Mortensen, A. (2008) Refugees as 'Others': Social and Cultural Citizenship Rights for Refugees in New Zealand Health Services. [PhD Thesis]. Auckland: Massey University. 
mrs kinpaisby. (2008). Taking Stock of Participatory Geographies: Envisioning the Communiversity. Transactions of the Institute of British Geographers, 33(3), 292- 299.

Munt, S. (2012). Journeys of Resilience: The Emotional Geographies of Refugee Women. Gender, Place \& Culture: A Journal of Feminist Geography, 19(5), 555-577.

Munt, S. (2016). Sensory Geographies and Defamiliarisation: Migrant Women Encounter Brighton Beach. Gender, Place \& Culture, 23(8), 1093-1106.

Murail, E. (2017). A Body Passes By: The Flâneur and the Senses in Nineteenth-Century London and Paris. The Senses and Society, 12(2), 162-176.

Murdoch, J. (2006). Post-Structuralist Geography: A Guide to Relational Space. London: Sage.

Nartey, Y., \& Sneyd, M. (2018). The Presenting Features of Melanoma in New Zealand: Implications for Earlier Detection. Australian and New Zealand Journal of Public Health, $42(6), 567-571$.

Nelson City Council. (2018). Council Boundary. Retrieved from http://www.nelson.govt.nz/council/council-structure/unitary-authority/council-boundary/

Nelson, L. (1999). Bodies (and Spaces) Do Matter: The Limits of Performativity. Gender, Place and Culture, 6, 331-353.

Nelson Marlborough Health. (2016). Healthy Homes. Retrieved from https://www.nmdhb.govt.nz/public-health-service/a-z-public-health-topics/healthy-homes/

Nelson Multicultural Council. (2012). Settling In: Refugees in Nelson. [Report]. Nelson: Author.

New Zealand Foreign Affairs and Trade. (2018). Myanmar. Retrieved from https://www.mfat.govt.nz/en/countries-and-regions/south-east-asia/myanmar/

New Zealand Government. (2018a). He Ara Oranga: Report of the Government Inquiry into Mental Health and Addiction. Wellington: Author.

New Zealand Government. (2018b). Refugee Quota Increases to 1500 in 2020. Retrieved from https://www.beehive.govt.nz/release/refugee-quota-increases-1500-2020

New Zealand Immigration. (2018a). Information for Refugees Settling in New Zealand. Retrieved from https://www.immigration.govt.nz/audiences/supporting-refugees-andasylum-seekers/information-for-refugees-settling-in-new-zealand 
New Zealand Immigration. (2018b). New Zealand Refugee Resettlement Strategy. Retrieved from https:/www.immigration.govt.nz/about-us/what-we-do/our-strategies-andprojects/refugee-resettlement-strategy

New Zealand Immigration. (2018c). Refugee and Protection. Retrieved from https://www.immigration.govt.nz/about-us/what-we-do/our-strategies-andprojects/supporting-refugees-and-asylum-seekers/refugee-and-protection-unit\#community

New Zealand Red Cross. (2013). Refugee Health and Wellbeing Project. Wellington: Author.

New Zealand Red Cross. (2018). Refugee Trauma Recovery. Retrieved from https://www.redcross.org.nz/what-we-do/in-new-zealand/refugee-programmes/refugeetrauma-recovery/

New Zealand Red Cross. (2020a). Pathways to Settlement. Retrieved from https://www.redcross.org.nz/what-we-do/in-new-zealand/migrationprogrammes/pathways-settlement/

New Zealand Red Cross (2020b). Taste of Cultures. Retrieved from https://www.redcross.org.nz/stories/new-zealand/taste-cultures/

New Zealand Trade and Enterprise. (2016). Nelson-Tasman. [Report]. Nelson: Author.

Nguyen, T., Ryan, R., \& Deci, E. (2018). Solitude as an Approach to Affective SelfRegulation. Personality and Social Psychology Bulletin, 44(1), 92-106.

NIWA. (2018). Nelson and Tasman. Retrieved from https://www.niwa.co.nz/node/110338

Noble, G. (2009). Everyday Cosmopolitanism and the Labour of Intercultural Community. In A., Wise, \& S. Velayutham (Eds.), Everyday Multiculturalism (pp. 46-65). Basingstoke: Palgrave Macmillan.

Novella, $\quad$ S. $\quad$ (2012). $\quad$ Earthing. $\quad$ Retrieved from https://theness.com/neurologicablog/index.php/earthing/

Oakley, A. (2016). Interviewing Women Again: Power, Time and the Gift. Sociology, 50(1), $195-213$.

O'Connell, T. (2019). Nelson PhD Student Explores Refugees' Emotional Attachments in Exhibition. Retrieved from https://www.stuff.co.nz/nelson-mail/news/111387230/nelsonphd-student-explores-refugees-emotional-attachments-in-exhibition 
O’Connor, R. (2014). Refugee Youth Resettlement Report. Hamilton: New Zealand Red Cross Refugee Services.

Oh, S. (2012). Photofriend: Creating Visual Ethnography with Refugee Children. Area, 44(3), 282-288.

Oldrup, H. H., \& Carstensen T. A. (2012). Producing Geographical Knowledge through Visual Methods. Geografiska Annaler: Series B, Human Geography, 94(3), 223-237.

Oliveira, E., Meyers, S., \& Vearey, J. (2016). Queer Crossings: A Participatory Arts-Based Project. Johannesburg, South Africa: The MoVE Project.

Oman, D., \& Thoresen, C. (2003). Spiritual Modelling: A Key to Spiritual and Religious Growth? International Journal for the Psychology of Religion, 13, 149-165.

Online Etymology Dictionary. (2020). Dis-. Retrieved from https://www.etymonline.com/word/dis-

O’Neill, M., \& Hubbard, P. (2010). Walking, Sensing, Belonging: Ethno-Mimesis as Performative Praxis. Visual Studies, 25(1), 46-58.

O’Regan, H. (2001). Ko Tahu, Ko Au: Käi Tahu Tribal Identity. Christchurch: Horomaka Publishing.

Oxford Burma Alliance. (2018). Learn About Burma. Retrieved from http://www.oxfordburmaalliance.org/learn-about-burma.html

Ozkul, D. (2013). 'You're Virtually There': Mobile Communication Practices, Locational Information Sharing and Place Attachment. First Monday, 18, 1-14.

Paasi, A. (2004). Place and Region: Looking through the Prism of Scale. Progress in Human Geography, 28(4), 536-546.

Pain, R. (2001). Gender, Race, Age and Fear in the City. Urban Studies, 38(5-6), 899-913.

Pain, R., \& Kindon, S. (2007). Participatory Geographies. Special Issue of Environment and Planning A, 39, 2807-2865.

Pain, R., \& Smith, S. (2008). Fear: Critical Geopolitics and Everyday Life. Farnham: Ashgate Publishing.

Pain, R., \& Smith, S. (2010). Geographies of Wellbeing. In S. Smith, R. Pain, Marston, S., \& J. P. Jones (Eds.), The SAGE Handbook of Social Geographies (pp. 198-515). London: SAGE Publications. 
Parr, J. (2010) Sensing Changes: Technologies, Environments, and the Everyday, 19532003. Vancouver: University of British Columbia Press.

Participatory Geographies Working Group. (2020). The PyGgRG Communifesto. Retrieved from https://pygyrg.org

Pennington, A., Orton, L., Nayak, S., Ring, A., Petticrew, M., Sowden, A., White, M., \& Whitehead, M. (2018). The Health Impacts of Women's Low Control in their Living Environment: A Theory-Based Systematic Review of Observational Studies in Societies with Profound Gender Discrimination. Health \& Place, 51, 1-10.

Pere, R. (1997). Te Wheke: A Celebration of Infinite Wisdom. Second Edition. Gisborne, New Zealand: Ao Ako Global Learning.

Phillips, R., Evans, B., \& Muirhead, S. (2015). Curiosity, Place and Wellbeing: Encouraging Place-Specific Curiosity as a 'Way to Wellbeing'. Environment and Planning A, 47, 23392354.

Philo, C., \& Wolch, J. (2001). The 'Three Waves' of Research in Mental Health Geography: A Review and Critical Commentary. Epidemiologia e Psichiatria Sociale, 10(4), 230-244.

Phipps, A. (2019a). The Well in Welcome: DeCarle Lecture 8. Retrieved from https://www.youtube.com/watch?v=HNGMS9sKXgU

Phipps, A. (2019b). The Rest of Labour. Retrieved from https://www.youtube.com/watch?v=lwug6lmaY84

Phipps, A., \& Kay, R. (2014). Languages in Migratory Settings: Place, Politics and Aesthetics. Language and Intercultural Communication, 14(3), 273-286.

Physicians for Human Rights. (2011). Life under the Junta: Evidence of Crimes against Humanity in Burma's Chin State. [Report]. Cambridge: Author.

Pink, S. (2005). Dirty Laundry: Everyday Practice, Sensory Engagement and the Constitution of Identity. Social Anthropology, 13(3), 275-290.

Pink, S. (2007). Doing Visual Ethnography. London: Sage.

Pink, S. (2015). Doing Sensory Ethnography: Second Edition. London: Sage.

Plate, S. B. (2012). The Skin of Religion: Aesthetic Mediations of the Sacred. Crosscurrents, 62(2), 162-180. 
Pleeging, E., Burger, M., \& Exel, J. V. (2019). The Relations between Hope and Subjective Well-Being: A Literature Overview and Empirical Analysis. Applied Research in Quality of Life, https://doi.org/10.1007/s11482-019-09802-4

Plunkett, D. (2011). On Place Attachments in Virtual Worlds. World Leisure Journal, 53(3), $168-178$.

Pocock, D. (1993). The Senses in Focus. Area, 25(1), 11-16.

Popke, J. (2006). Geography and Ethics: Everyday Mediations through Care and Consumption. Progress in Human Geography, 30(4), 504-512.

Popke, J. (2007). Geography and Ethics: Spaces of Cosmopolitan Responsibility. Progress in Human Geography, 31, 509-518.

Porteous, J. D. (1976). Home: The Territorial Core. Geographical Review, 66, 383-390.

Porteous, D. (1990). Landscapes of the Mind: Worlds of Sense and Metaphor. Toronto: University of Toronto Press.

Pottinger, L. (2017). Planting the Seeds of a Quiet Activism. Area 49(2), 215-222.

Potts, T. (2015). Rhythmanalysis: Space, Time and Everyday Life. The Journal of Architecture, 20(3), 550-554.

Probyn, E. (1996). Outside Belongings. New York: Routledge.

Procupez, V. (2015). The Need for Patience: The Politics of Housing Emergency in Buenos Aires. Current Anthropology, 56(11), 555-565.

Proshansky, H., Fabien, A., \& Kaminoff, R. (1983). Place-Identity: Physical World Socialization of the Self. Journal of Environmental Psychology, 3, 57-83.

Pussetti, C. (2013). 'Woundscapes': Suffering, Creativity and Bare Life - Practices and Processes of an Ethnography-Based Art Exhibition. Critical Arts: South-North Cultural and Media Studies, 27(5), 569-586.

Putnam, R. (2007). E Pluribus Unum: Diversity and Community in the Twenty-First Century. The 2006 Johan Skytte Prize Lecture. Scandinavian Political Studies, 30(2), 137174.

Radio New Zealand. (2020). Christchurch Terror Attacks. Retrieved from https://www.rnz.co.nz/news/chch-terror

Rameka, L. (2018). A Māori Perspective of Being and Belonging. Contemporary Issues in Early Childhood, 19(4), 367-378. 
Ramos, M. (2004). Drawing the Lines: The Limitations of Intercultural Ekphrasis. In S. Pink, L. Kurti, \& A. Afonso (Eds.), Working Images: Visual Research and Representation in Ethnography (pp. 147-156). London: Routledge.

Randolph, T. G. (1987). Environmental Medicine: Beginnings and Bibliographies of Clinical Ecology. Fort Collins CO: Clinical Ecology Publications.

RASNZ. (2018). Refugee Health and Wellbeing. Retrieved from https://rasnz.co.nz/

Ratnam, C. (2018). Creating Home: Intersections of Memory and Identity. Geography Compass, 12(2), e12363.

Reid, J., Rout, M., Tau, T. M., \& Smith, C. (2017). The Colonising Environment: An Aetiology of the Trauma of Settler Colonisation and Land alienation on Ngai Tahu Whanau. Christchurch: University of Canterbury.

Reimer, A. (2008). It's a Risky Business [Interview with Tim Winton]. Retrieved from https://www.smh.com.au/entertainment/books/its-a-risky-business-20080425-gdsaxa.html

Relph, E. (1976). Place and Placelessness. London: Pion.

Rishbeth, C., \& Powell, M. (2013). Place Attachment and Memory: Landscapes of Belonging as Experienced Post-Migration. Landscape Research, 38(2), 160-178.

Rishbeth, C., Blachnicka-Ciacek, D., \& Darling, J. (2019). Participation and Wellbeing in Urban Greenspace: 'Curating Sociability' for Refugees and Asylum Seekers. Geoforum, 106, $125-134$.

Rodaway, P. (1994). Sensuous Geographies: Body, Sense and Place. London: Routledge.

Room to Heal. (2018). Therapeutic Gardening. Retrieved from http://roomtoheal.org/approach/therapeutic-gardening/

Rosbrook, B., \& Schweitzer, R. (2010). The Meaning of Home for Karen and Chin Refugees from Burma: An Interpretative Phenomenological Approach. European Journal of Psychotherapy and Counselling, 12(2), 159-172.

Rose, G. (1993). Feminism and Geography: The Limits of Geographical Knowledge. Minnesota: The University of Minnesota Press.

Rose, G. (2012). Visual Methodologies: An Introduction to Researching with Visual Materials. 3rd ed. London: Sage Publications.

Rose, N. (2002). Powers of Freedom, Reframing Political Thought. Cambridge: Polity. 
Royal Geographical Society with IBG. (2018). Ask the Geographer: How is PlaceAttachment and Belonging Managed in Refugee Re-Settlement? Sara Kindon and Amber Kale. Retrieved from https://poddtoppen.se/podcast/1196746426/ask-the-geographer/howis-place-attachment-and-belonging-managed-in-refugee-re-settlement-sara-kindon-andamber-kale

Rubenstein, R. (2001). Home Matters: Longing and Belonging, Nostalgia and Mourning in Women's Fiction. New York: Palgrave.

Sampson, R., \& Gifford, S. M. (2010). Place-Making, Settlement and Well-Being: The Therapeutic Landscapes of Recently Arrived Youth with Refugee Backgrounds. Health Place, 16(1), 116-31.

Saxbe, D. E., \& Repetti, R. (2010). No Place like Home: Home Tours Correlate with Daily Patterns of Mood and Cortisol. Personality and Social Psychology Bulletin 36(1) 71-81.

Scannell, L., \& Gifford, R. (2010). Defining Place Attachment: A Tripartite Organizing Framework. Journal of Environmental Psychology, 30, 1-10.

Scannell, L., \& Gifford, R. (2016). Place Attachment Enhances Psychological Need Satisfaction. Environment and Behavior, 49(4), 359-389.

Scarlis, C. (2010). Chin Cultural Profile. Retrieved from http://ethnomed.org/culture/chin\#section-13

Schwab, K. (2013). The Global Repetitiveness Report 2013-14. Geneva: World Economic Forum.

Scoffham, S., \& Barnes, J. (2011). Happiness Matters: Towards a Pedagogy of Happiness and Well-Being. Curriculum Journal, 22(4), 535-548.

Seamon, D. (1980). Body-Subject, Time-Space Routines, and Place-Ballets. In A. Buttimer, \& D. Seamon (Eds.), The Human Experience of Space and Place (pp. 148-165). London, UK: Croom Helm.

Seamon, D. (2014). Lived Emplacement and the Locality of Being: A Return to Humanistic Geography? In S. Aitken, \& G., Valentine, Approaches to Human Geography: Philosophies, Theories, People and Practices (pp. 35-48). New York: Sage Publications.

Seamon, D. (2018). Life Takes Place: Phenomenology, Lifeworlds, and Place Making. London: Routledge. 
Searle, W., Prouse, E., L’Ami, E., Gray, A., \& Grunner, A. (2012). New Land, New Life: Long-Term Settlement of Refugees in New Zealand. Main Report. Wellington: Ministry of Business, Innovation and Employment.

SensoryCo. (2020). Experiential Spaces. Retrieved from https://sensoryco4d.com/\#

Sensory Journeys. (2017). Sensory Journeys. Retrieved from http:/www.sensoryjourneys.net/

Seremetakis, C. N. (1993). The Memory of the Senses: Historical Perception, Commensal Exchange, and Modernity. Visual Anthropology Review, 9(2), 2-18.

Shan Women's Action Network and the Shan Human Rights Foundation. (2002). License to Rape. Chiang Mai: Authors.

Shirlow, P., \& Pain, R. (Eds.) (2003). The Geographies and Politics of Fear [Special issue]. Capital and Class, 27(2).

Sibley, D. (1981). Outsiders in Urban Societies. New York: St. Martin's Press.

Simmel, G. (1997 [1907]). Simmel on Culture: Selected Writings. D. Frisby \& E. Featherstone (Eds.), London: Sage.

Slow Research Lab. (2018). Encounter: In-Situ Dialogues. Retrieved from http://slowlab.net/ENCOUNTER/Encounter-IN-SITU-DIALOGUES

Smith, M. (1994). Ethnic Groups in Burma: Development, Democracy and Human Rights. [Report]. London: Anti-Slavery International.

Smith, N. \& O’Keefe, P. (1996 [1980]). Geography, Marx and the Concept of Nature. In J. Agnew, D. Livingstone, and A. Rogers (Eds.), Human Geography: An Essential Anthology (pp. 282-295). Oxford: Blackwell.

Smith, S. (1994). Soundscape. Area, 26(3), 232-240.

Snyder, C. R., Rand, K. L., \& Sigmon, D. R. (2002). Hope Theory: A Member of the Positive Psychology Family. In Snyder, C. R. \& Lopez, S. J. (Eds.), Handbook of Positive Psychology (pp. 411-419). New York: Oxford University Press.

Sointu, E. (2005). The Rise of an ideal: Tracing Changing Discourses of Wellbeing. The Sociological Review, 53, 255-274.

Southern Cross. (2019). Iron Deficiency Anaemia. Retrieved from https://www.southerncross.co.nz/group/medical-library/iron-deficiency-anaemia 
Spaaij, R., Broerse1, J., Oxford, S., Luguetti, C., McLachlan, F., McDonald, B., Klepac, B., Lymbery, L., Bishara, J., \& Pankowiak, A. (2019). Sport, Refugees, and Forced Migration: A Critical Review of the Literature. Frontiers in Sports and Active Living, 1(47), 1-18.

Spinney, J. (2006). A Place of Sense: A Kinesthetic Ethnography of Cyclists on Mont Ventoux. Environment and Planning D: Society and Space, 24(5), 709-732.

Spinney, J. (2015). Close Encounters? Mobile Methods, (Post)Phenomenology and Affect. Cultural Geographies, 22(2), 231-246.

Staeheli, L. (2010). Political Geography: Where's Citizenship? Progress in Human Geography, 35(3), 393-400.

Stats NZ. (2019a). Losing our Religion. Retrieved from https://www.stats.govt.nz/news/losing-our-religion

Stats NZ. (2019b). Population Clock. Retrieved from http://archive.stats.govt.nz/tools_and_services/population_clock.aspx

Stats NZ. (2020). Nelson Region. Retrieved from https://www.stats.govt.nz/tools/2018census-place-summaries/nelson-region

Steadman, R. (2006). Understanding Place Attachment among Second Home Owners. American Behavioral Scientist, 50(2), 187-205.

Stein, B., Stanford, T., \& Rowland, B. (2009). The Neural Basis of Multisensory Integration in the Midbrain: Its Organization and Maturation. Hearing Research, 258(1-2), 4-15.

Steiner, R. (1981). Man's Twelve Senses in Their Relation to Imagination, Inspiration, and Intuition. Anthroposophical Review, 3(2), 12-19.

Stephens, M. (2018). Doing Our Bit: The Campaign to Double the Refugee Quota. Wellington: Bridget Williams Books.

Stevens, Q. (2007). The Ludic City: Exploring the Potential of Public Spaces. London: Routledge.

Stewart, S. (1993). On Longing: Narratives of the Miniature, the Gigantic, the Souvenir, the Collection. Durham NC: Duke University Press.

Stokols, D., \& Shumaker, S. A. (1981). People in Places. A Transactional View of Settings. In J. Harvey (Ed.), Cognition, Social Behavior and the Environment (pp. 441-488). Hillsdale, NJ: Lawrence Erlbaum Associates.

Stoller, P. (1997). Sensuous Scholarship. Philadelphia: University of Pennsylvania Press. 
Strang, A., \& Ager, A. (2010). Refugee Integration: Emerging Trends and Remaining Agendas. Journal of Refugee Studies, 23(4), 589-607.

Suckling, L. (2016). New Zealand's Flawed Ideal of Masculinity. Retrieved from https://www.stuff.co.nz/life-style/life/83966192/new-zealands-flawed-ideal-of-masculinity

Sutton, D. (2010). Food and the Senses. Annual Review of Anthropology, 39, 209-223.

Swanton, D. (2016). Encountering Keighley: More-than-human Geographies of Difference in a Former Mill Town. In J. Darling \& H. Wilson (Eds.), Encountering the City: Urban Encounters from Accra to New York (pp. 111-132). London: Routledge.

Swe, Y. Y. (2013). Mobility Encounter: The Narratives of Burmese Refugees in Norway. Norsk Geografisk Tidsskrift - Norwegian Journal of Geography, 67(4), 229-238.

Synnott, A. (1993). The Body Social. London Routledge.

Tardis, M. (2009). Another Story from the "Refugee Crisis": Resettlement in Small Towns and Rural Areas in France. Paris: 'Etudes De l'Ifri.

Taylor, H. (2013). Refugees, the State and the Concept of Home. Refugee Survey Quarterly, 32(2), 130-152.

Taylor, R. B., Gottfredson, S. D., \& Brower, S. (1985). Attachment to Place: Discriminant Validity, and Impacts of Disorder and Diversity. American Journal of Community Psychology, 13, 525-542.

Tee Noe. (2014). Karen Resistance Poetry. [Translated and introduced by Violet Cho]. Transnational Literature, 6(2), 2-3.

Te Pou o te Whakaaro Nui. (2018). Sensory Modulation. Retrieved from https://www.tepou.co.nz/initiatives/sensory-modulation/103

Temple, B., \& Edwards, R. (2006). Limited Exchanges: Approaches to Involving People Who do not Speak English in Research and Service Development. In Temple, B., \& R. Moran. (Eds.), Doing Research with Refugees: Issues and Guidelines (pp. 37-54). Bristol: Policy Press.

The Economist. (2016). Should you Say Myanmar or Burma? Retrieved from https://www.economist.com/the-economist-explains/2016/12/20/should-you-say-myanmaror-burma

The Key to Life Charitable Trust. (2020). Spreading Hope from Bluff to Cape Reinga. Retrieved from https://keytolife.org.nz 
Thomas, A. (2016). Accelerating Threats from Climate Change: Disasters and Displacement in Myanmar. Retrieved from https://www.refugeesinternational.org/reports/2016/myanmar

Thomas, R., Chiarelli-Helminiak, C., Ferraj, B., \& Barrette, K. (2015). Building Relationships and Facilitating Immigrant Community Integration: An Evaluation of a Cultural Navigator Program. Evaluation and Program Planning, 55, 77-84.

Thoo Lei Wild Grass. (2011). Hoping to See You Again. Retrieved from https://www.poetryinternationalweb.net/pi/site/cou_article/item/23184/Mother-died-andtime-passed/en

Thrift, N. (1996). Spatial Formations. Thousand Oaks, CA: Sage.

Thrift, N. (2000). Afterwords. Environment and Planning D: Society and Space, 18, 213255.

Thrift, N. (2004). Intensities of Feeling: Towards a Spatial Politics of Affect. Geografiska Annaler: Series B, Human Geography, 86, 57-78.

Thrift, N. (2008). Non-Representational Theory: Space, Politics, Affect. Abbingdon: Routledge.

Tolia-Kelly, D. (2008). Motion/Emotion: Picturing Translocal Landscapes in the Nurturing Ecologies Research Project. Mobilities, 3(1), 117-140.

Tonkin, E. (1992). Narrating Our Pasts: The Social Construction of Oral History. Cambridge: Cambridge University Press.

Trnka, S., Dureau, C., \& Park, J. (2013). Senses and Citizenships: Embodying Political Life. New York: Routledge.

Tsoukala, A. (2016). Turning Immigrants into Security Threats: A Multifaceted Process. In G. Lazaridis (Ed.), Security, Insecurity and Migration in Europe. Abingdon: Routledge.

Tuan, Y. (1974). Topophilia: A Study of Environmental Perception, Attitudes and Values. Englewood Cliffs, N.J: Prentice Hall.

Tuan, Y. (1976). Humanistic Geography. Annals of the Association of American Geographers, 66(2), 266-276.

Tuan, Y. (1977). Space and Place: The Perspective of Experience. Minneapolis: University of Minnesota Press.

Tuan, Y. (1991). Language and the Making of Place: A Narrative-Descriptive Approach. Annals of the Association of American Geographers, 81(4), 684-696. 
Tuan, Y. (1993). Passing Strange and Wonderful: Aesthetics, Nature, and Culture. Washington DC: Island Press, Shearwater Books.

Twigger-Ross, C. \& Uzzell, D. (1996). Place and Identity Processes. Journal of Environmental Psychology, 16, 205-220.

Ulrich, R. S. (1984). View through a Window May Influence Recovery from Surgery. Science, 224(4647), 420-421.

UNESCO. (2017). Displaced Person / Displacement. Retrieved from http://www.unesco.org/new/en/social-and-human-sciences/themes/internationalmigration/glossary/displaced-person-displacement/

UNHCR. (1996). Convention and Protocol Relating to the Status of Refugees. [Report]. Geneva: Author.

UNHCR. (2014). Kayah State Profile. Geneva: Author.

UNHCR. (2016). Global Trends: Forced Displacement in 2016. Retrieved from http://www.unhcr.org/globaltrends2016/

UNHCR. (2017). Figures at a Glance. Retrieved from http://www.unhcr.org/figures-at-aglance.html

UNHCR. (2019). Worldwide Displacement Tops 70 Million, UN Refugee Chief Urges

Greater Solidarity in Response. Retrieved from

https://www.unhcr.org/uk/news/press/2019/6/5d03b22b4/worldwide-displacement-tops-

70-million-un-refugee-chief-urges-greater-solidarity.html

United States Institute of Peace. (2018). What's in a Name: Burma or Myanmar? Retrieved from https://www.usip.org/blog/2018/06/whats-name-burma-or-myanmar

University of Glasgow. (2019). UNESCO Rila Spring School 2019. The Arts of Integrating: Labouring and Resting. Retrieved from https://www.gla.ac.uk/research/az/unesco/artsandculturalengagement/artshub/springschool/ Urry, J. (2003). Global Complexity. Cambridge: Polity.

Urry, J. (2007). Mobilities. Cambridge: Polity.

Urry, J. (2012). Social Networks, Mobile Lives and Social Inequalities. Journal of Transport Geography, 21, 24-30.

Valentine, G. (2005). Tell Me About...: Using Interviews as a Research Methodology. In Flowerdew, R., \& D. Martin. (Eds.), Methods in Human Geography: A Guide for Students 
Doing a Research Project. Second Edition (pp. 110-127). London: Pearson Education Limited.

Valle, M., Huebner, E. \& Suldo, S. (2006). An Analysis of Hope as a Psychological Strength. Journal of School Psychology, 44(5), 393-406.

Valli, C. (2015). A Sense of Displacement: Long-time Residents' Feelings of Displacement in Gentrifying Bushwick, New York. International Journal of Urban and Regional Research, 39(6), 1191-1208.

Vannini, P. (2015). Non-representational Ethnography: New Ways of Animating Lifeworlds. Cultural Geographies, 22(2), 317-327.

Verderber, S. (1986). Dimensions of Person-Window Transactions in the Hospital Environment. Environment and Behavior, 18(4), 450-466.

Victoria University of Wellington. (2020). Connecting With New Places. Retrieved from https:/www.wgtn.ac.nz/news/2020/02/connecting-with-new-

places?fbclid=IwAR0ZQFjaPCw6249CZ4sJyAvRD65imrBwXootiyqSQFup1x_tqnurH5c YNCk

Volkan, V. D. (1999). Nostalgia as a Linking Phenomenon. Journal of Applied Psychoanalytic Studies, 2, 169-179.

Volker, S., \& Kistemann, T. (2011). The Impact of Blue Space on Human Health and WellBeing - Salutogenetic Health Effects of Inland Surface Waters: A Review. International Journal of Hygiene and Environmental Health, 214, 449-460.

Waite, L., \& Cook, J. (2011). Belonging Among Diasporic African Communities in the UK: Plurilocal Homes and Simultaneity of Place Attachments. Emotion, Space and Society, $4(4), 238-248$.

Waite, L., Valentine, G., \& Lewis, H. (2014). Multiply Vulnerable Populations: Mobilizing a Politics of Compassion from the 'Capacity to Hurt'." Social and Cultural Geography, 15, 313-31.

Ward, C., Lescelius, J., Jack, A., Naidu, R., \& Weinberg, E. (2018). Meeting the Needs and Challenges of Migrants and Former Refugees in the Nelson and Tasman Regions. [Report]. Wellington: Victoria University of Wellington.

Welcoming International. (2020). Creating a World Where Everyone Feels at Home. Retrieved from https://welcominginternational.org 
West, M. (1986). Landscape Views and Stress Response in the Prison Environment. Landscape Architecture. Seattle: University of Washington.

Westin, A. (1967). Privacy and Freedom. New York: Athenuem.

Westin, K. (2015). Place Attachment and Mobility in City Regions. Population, Space and Place, 22(8), 722-735.

Whatmore, S. (2002). Hybrid Geographies: Natures, Cultures, Spaces. London: Sage.

Whatmore, S. (2006). Materialist Returns: Practising Cultural Geography in and for a MoreThan-Human World. Cultural Geographies, 13(4), 600-609.

Williams, A. (1999). Therapeutic Landscapes: The Dynamic between Place and Wellness. Lanham, MD: University Press of America.

Wilson, H. (2017). On Geography and Encounter: Bodies, Borders, and Difference. Progress in Human Geography, 41(4), 451-471.

Wilson, K. (2003). Therapeutic Landscapes and First Nations Peoples: An Exploration of Culture, Health and Place. Health \& Place, 9(2), 83-93.

Wise, A. (2005). Hope and Belonging in a Multicultural Suburb. Journal of Intercultural Studies, 26(1-2), 171-186.

Wood, P. B., McGrath, S., \& Young, J. (2012). The Emotional City: Refugee Settlement and Neoliberal Urbanism in Calgary. Journal of International Migration and Integration, 13, 21-37.

Wolch, J. (2002). Anima Urbis. Progress in Human Geography, 26, 721-742.

Wolch, J., Byrne, J., \& Newell, J. (2014). Urban Green Space, Public Health, and Environmental Justice: The Challenge of Making Cities 'Just Green Enough'. Landscape and Urban Planning, 125, 234-244.

World Health Organization. (2005). Promoting Mental Health. Geneva: Author.

Wright, S. (2008). Practising Hope: Learning from Social Movement Strategies in the Philippines. In R. Pain, \& S. Smith (Eds.), Fear: Critical Geopolitics and Everyday Life (pp. 223-234). Farnham: Ashgate Publishing.

Wright, S. (2015). More-Than-Human, Emergent Belongings: A Weak Theory Approach. Progress in Human Geography, 39(4), 391-411.

Wynne-Jones, S., North, P., \& Routledge, P. (2015). Practising Participatory Geographies: Potentials, Problems and Politics. Area, 47(3), 218-221. 
Yuval-Davis, N. (2011). Power, Intersectionality and the Politics of Belonging. FREIA Working Paper Series No. 75. Aalborg: FREIA.

Zajonc, R. B. (1968). Attitudinal Effects of Mere Exposure. Journal of Personality and Social Psychology, 9(2), 9-27.

Zuss, M. (2012). The Practice of Theoretical Curiosity. New York: Springer. 